\title{
Design of GaN-based microwave components and application to novel high power reconfigurable antennas
}

By

\author{
Abdelaziz HAMDOUN \\ degree of \\ Doctor of Philosophy \\ In \\ Electrical Engineering \\ Department of Electronics \\ Carleton University \\ Ottawa, Ontario, Canada \\ (C) October, 2016
}

A thesis submitted to the Faculty of Graduate and Postdoctoral Affairs in partial fulfillment of the requirements for the

Abdelaziz HAMDOUN 


\section{Professor Mohamed HIMDI}

University of Rennes 1, France, Thesis Supervisor

Professor Langis ROY
Carleton University, Ottawa, Canada,
Thesis Supervisor

Professor Mustapha YAGOUB

University of Ottawa, Canada, Referee

\section{Professor Nathalie ROLLAND}

University of Lille 1, France, Referee

\section{Adjunct Professor Olivier LAFOND \\ University of Rennes 1, France, Examiner}

\section{Adjunct Professor Rony AMAYA}

Carleton University, Ottawa, Canada, Examiner

\section{Adjunct Professor Steve McGARRY}

Carleton University, Ottawa, Canada, Examiner

\section{Professor Barry SYRETT}

Carleton University, Canada, Thesis co-Supervisor

Professor Xavier CASTEL

University of Rennes 1, France, Chair of Member Committee 


\section{Table of Contents}

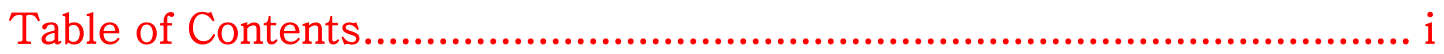

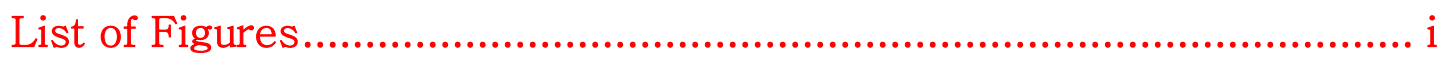

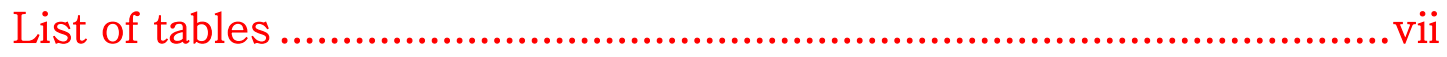

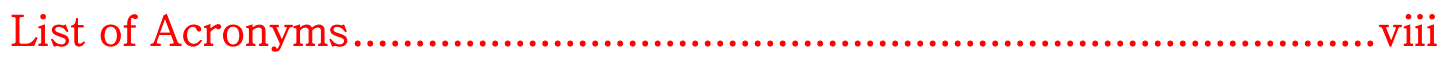

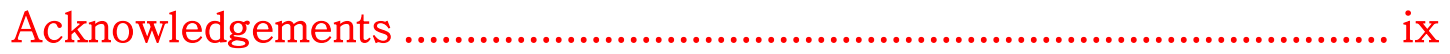

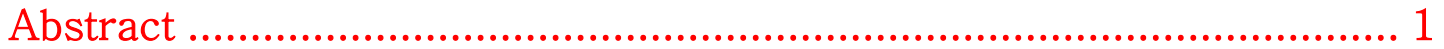

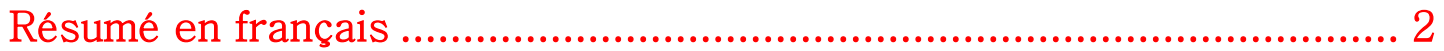

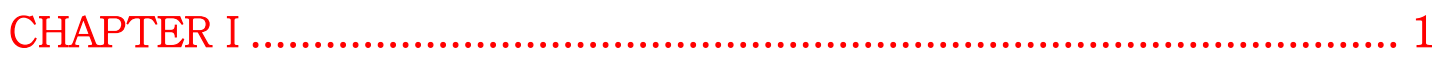

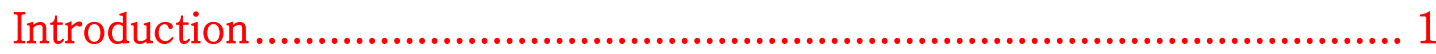

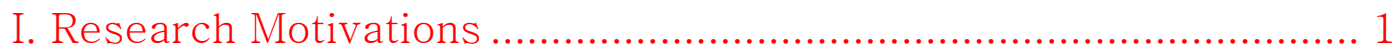

II. Objective of Research ................................................................. 5

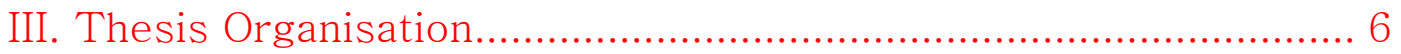

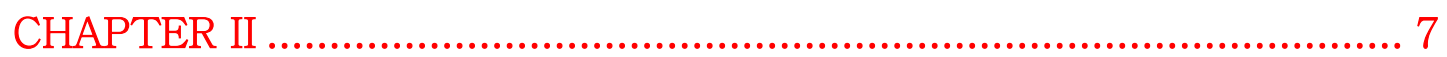

A Review of the Literature of Gallium Nitride Technology .................... 7

I. Gallium Nitride Technology ........................................................... 7

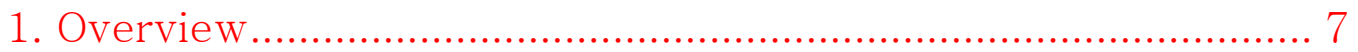

2. Choice of the Substrate .......................................................... 14

II. NRC GaN500 MMIC HEMT Process Features and Limitations.... 16

III. Use of GaN in Reconfigurable RF/Microwave Systems ............. 21

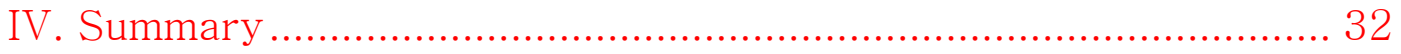

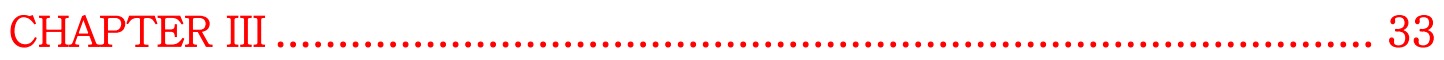

GaN Varactor and Switch Devices Modeling ....................................... 33

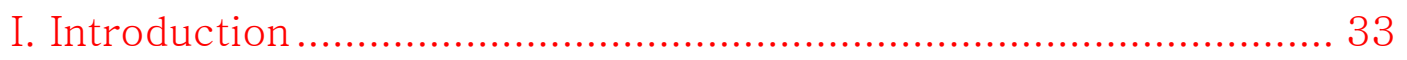

II. GaN-based Varactors Diodes Modeling ........................................ 36

1. Review of GaN HEMT for Power Varactors Diodes Applications 
2. Realized GaN HEMT-based Varactor Diodes........................... 46

3. GaN500v1 and GaN150v0 Varactor Devices ............................ 47

3.1 Small Signal Modeling............................................................ 48

3.1.1 Analysis of Equivalent Capacitance ............................. 52

3.1.2 Analysis of Equivalent Series resistance ...................... 55

3.1.3 Analysis of Q-Factor ……………............................... 56

4. GaN500v2 Varactor Devices ..................................................... 58

4.1 Small Signal Measurement ................................................ 58

4.1.1 Analysis of Equivalent Capacitance ……….................. 61

4.1.2 Analysis of Equivalent Series Resistance ...................... 65

4.2 Large Signal Measurements ................................................ 68

4.2.1 Analysis of Equivalent Capacitance .............................. 69

4.2.2 Analysis of Equivalent Series Resistance ….................. 71

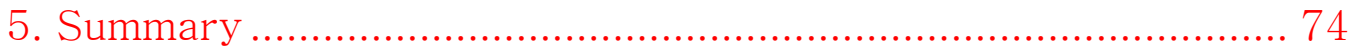

III. GaN-based Switch Devices Modeling ........................................ 75

1. Review of GaN HEMT for Power Switching Devices Applications

2. NRC GaN Switch Modeling …..................................................... 78

2.1 Proposed GaN Single Switch Model ..................................... 78

3. GaN500v2 Small Signal Switches Measurements..................... 82

3.1 Different Sizes Standalone Signal Switch Devices............... 82

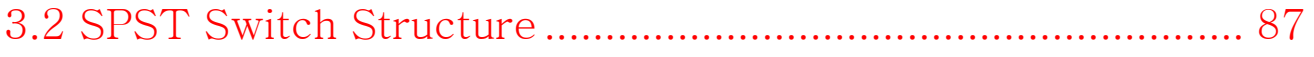

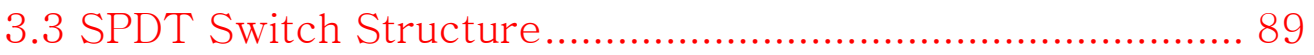

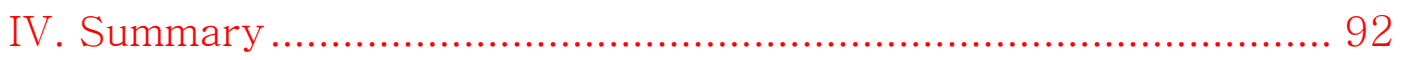

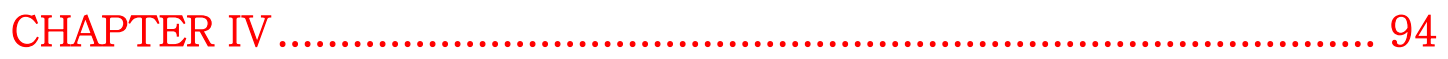

Realized GaN-Based Reconfigurable Circuits ...................................... 94 


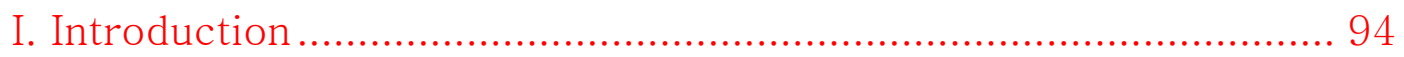

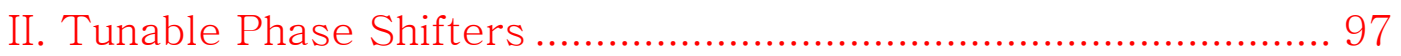

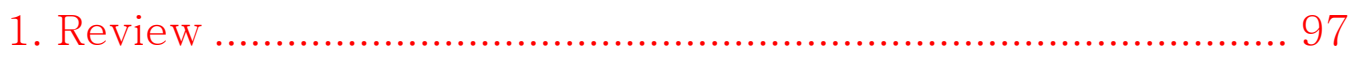

2. Design of Analog GaN-based Phase Shifter .......................... 105

2.1 Quadrature 3-dB 90 Hybrid Coupler................................ 108

2.1.1. Simulated Results and Discussion............................... 115

2.2 Phase Shifter Design ….................................................... 117

2.1.2. Simulated Results and Discussion............................... 119

III. GaN Reconfigurable 3-dB/90 Hybrid Coupler.......................... 122

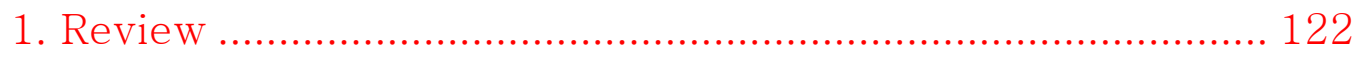

2. Design of Reconfigurable 3-dB/90 Coupler .......................... 124

2.1 Simulated Results and Discussion...................................... 125

IV. GaN-based Oscillator ............................................................. 129

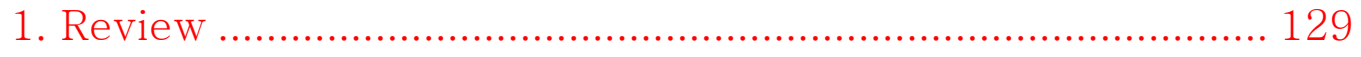

2. Design of GaN-based Oscillators …......................................... 132

2.1 Feedback Oscillator Topology ............................................ 133

2.2 Power Amplifier Design......................................................... 133

2.2.1 PA Design Procedure ….................................................. 133

2.2.2 PA Simulated Results ................................................... 139

2.2.3 PA Measured Results .................................................... 140

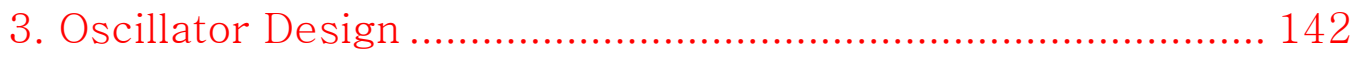

3.1 Start-up Oscillation Condition............................................ 142

3.2 Phase Noise in Oscillator .................................................. 144

3.3 Fixed Frequency Oscillator (FFO) ..................................... 145

3.3.1 Simulation Results ...................................................... 145

3.3.2 Measurements Results ............................................. 148

3.4 Tunable Frequency Oscillator (TFO) ................................ 149 
3.4.1 Simulation Results ..................................................... 149

3.4.2 Measurement Results ................................................. 152

V. Frequency Reconfigurable Active Antennas............................. 153

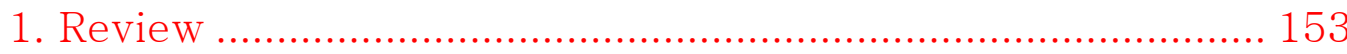

2. Reconfigurable Annular Slot Antenna using GaN Varactor..... 154

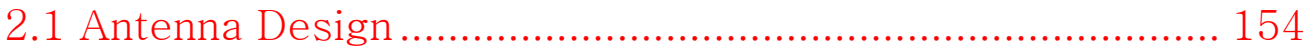

2.2 Simulated Return Loss and Radiation Pattern .................... 155

2.3 Measured Return Loss and Radiation Pattern ................... 158

3. Reconfigurable Aperture Coupled Slot Antenna using GaN

Varactor 162

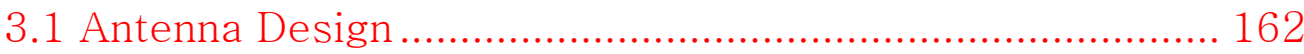

3.2 Simulated Return Loss and Radiation Pattern ................... 163

3.3 Measured Return Loss and Radiation Pattern .................... 167

4. Beam Steering Patch Antenna Arrays using GaN SPDT Switch

Structure 170

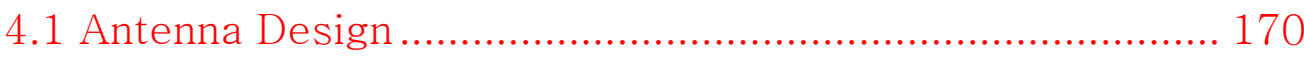

4.2 Simulated Return Loss and Radiation Pattern ................... 174

4.3 Measured Return Loss and Radiation Pattern .................... 178

4.4 Measured Insertion Loss and Isolation of the Feeding System

4.4.1 Small Signal Measurements ...................................... 181

4.4.2 Large Signal Measurements ...................................... 184

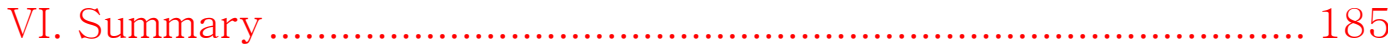

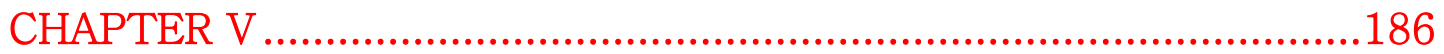

Conclusion and Future Work .......................................................186

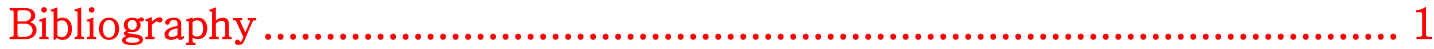


Table of Contents 



\section{List of Figures}

Figure II.1: Most used Semiconductor Technologies and their Frequency/Power limits [6] ................ 9

Figure II.2: Current due to trapping effect and thermal effect [18] ................................................. 11

Figure II.3: A sketch of an AlGaN/ GaN HEMT structure with trapped electrons [19] ...................... 12

Figure II.4: Cross-Section of HEMTs (Not Drawn to Scale) ........................................................... 13

Figure II.5: Cross-sectional view of HFET, showing process sequence (not to scale) [31]................... 19

Figure II.6: Photo of a T/R-Module front-end with GaN MMIC chips [43] .........................................22

Figure II.7: Measured output power versus frequency of the GaN MMIC DA at ..............................223

Figure II.8: Measured power performance of the GaN MMIC HPA at $11 \mathrm{GHz}$...............................22

Figure II.9: Measured gain and noise figure versus frequency of the GaN MMIC ............................24

Figure II.10: (Left) A schematic view and (Right) a photograph of the Fabricated T/R module using

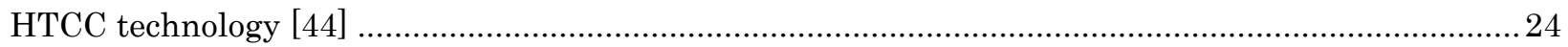

Figure II.11: Measured Pout of fabricated T/R module as a function of frequency, biased at $30 \mathrm{~V}$ [44] .25

Figure II.12: Measured S-parameters of GaN LNA MMIC (Vds = 15 V) [44] .................................25

Figure II.13: Photograph of the fabricated amplifier [45] ...............................................................26

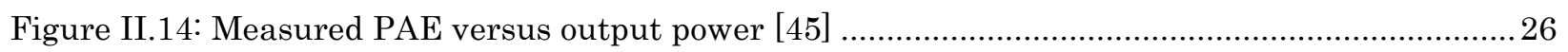

Figure II.15: Measured gain versus output power [45] .................................................................2 27

Figure II.16: The concept of impedance matching conditions of the band and class reconfigurable power

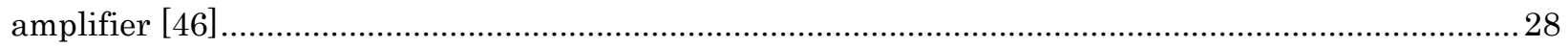

Figure II.17: Photograph of the fabricated class-reconfigurable power amplifier [46] ......................28

Figure II.18: Simulated gain verses input power at different matching states [46] ........................28

Figure II.19: Simulated output power verses input power at different matching states [46] .............29

Figure II.20: Simulated power added efficiency verses input power at different matching states [46] .29

Figure II.21: (Left) Top and cross sectional view and (Right) Picture of the packaged PA [47] ........... 30

Figure II.22: Acetone configuration: measurements at $2.3 \mathrm{GHz}$, simulations at $2.4 \mathrm{GHz}$ [47] ...........30

Figure II.23: Air configuration: measurements at $5.7 \mathrm{GHz}$, simulations at $5.8 \mathrm{GHz}$ [47] .................. 31

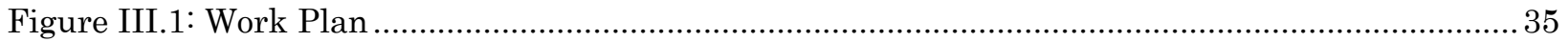

Figure III.2: C-V characteristics of devices with different electrode length $($ spacing $=1 \mathrm{um}$, width $=50$ um) [53]

Figure III.3: Extracted capacitance (black line) and resistance (white line) of the MSM varactor over the entire bias range at $1.1 \mathrm{GHz}$. The inserted schematic shows the first-order equivalent circuit of the MSM varactor [54]. .38 


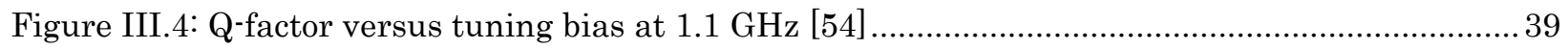

Figure III.5: A Schematic diagram of the AlGaN/GaN-based varactor [55] ...................................... 40

Figure III.6: Measured C-V curves of the untreated and sulfur-treated AlGaN/GaN-based MSM-2DEG

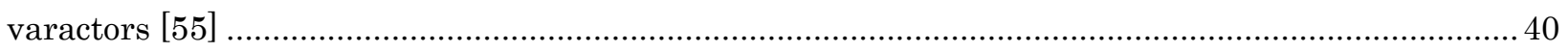

Figure III.7: Schematic diagram of the AlGaN/GaN MSM-2DEG varactor of the samples prepared in

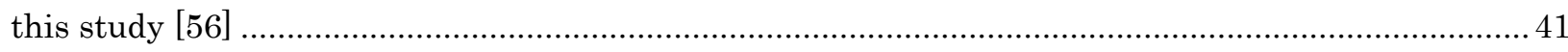

Figure III.8: Capacitance-Voltage (C-V) characteristics of varactors with different electrode areas (a) without any dielectric film, (b) with $\mathrm{SiO} 2$ film, (c) with Gd2O3 film, and (d) with Si3N4 film. Measurement frequency $=1 \mathrm{MHz}$, electrode area $=37922,85224,151122$, and $236472 \mu \mathrm{m} 2$,

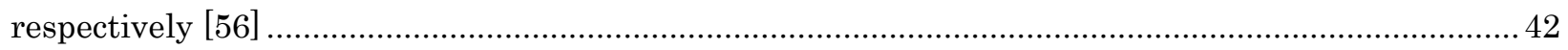

Figure III.9: Schematic Cross-sectional view of the GaN Schottky Varactor [57] ..............................43

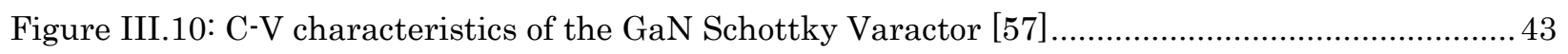

Figure III.11: Schematic diagram of a GaN-based varactor and its layer structure [58] ................... 44

Figure III.12: C-V curves measured for the samples with area of $5 \times 10-5 \mathrm{~cm} 2$ at $\mathrm{f}=1 \mathrm{MHz}$ [58] ....44

Figure III.13: Capacitance-voltage curves for (a) single diode structure and (b) two anti-parallel diode

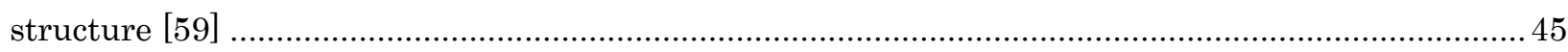

Figure III.14: $\mathrm{Q}$ factor versus reverse external bias for an anti-parallel varactor at $\mathrm{f}=1 \mathrm{GHz}, 2 \mathrm{GHz}$

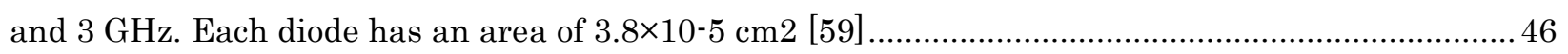

Figure III.15: Schematic cross-section of HEMT, showing contacts and epitaxial structures, and

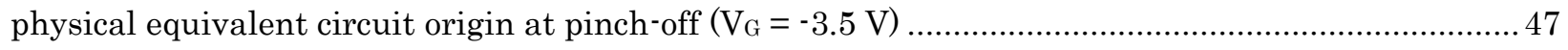

Figure III.16: W40L500v1 Device in Microwave Probe Test ............................................................ 48

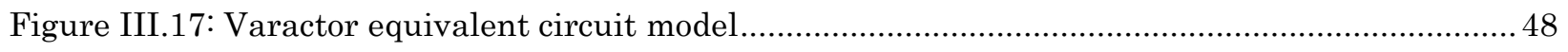

Figure III.18: Measured (lines) and Simulated (Dot) S11-parameter for G W40L0.5 ........................50

Figure III.19: Measured (lines) and Simulated (Dot) S11-parameter for W100L0.5 ..........................50

Figure III.20: Measured (lines) and Simulated (Dot) S11-parameter for W50L0.15 ...........................51

Figure III.21: Measured (lines) and Simulated (Dot) S11-parameter for W100L0.15 ........................51

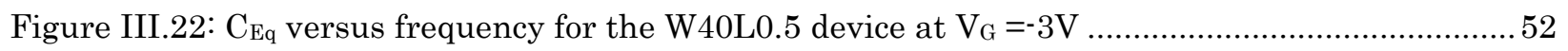

Figure III.23: Extracted and Simulated $\mathrm{C}_{\mathrm{Eq}}$ versus $\mathrm{V}_{\mathrm{G}}$ for GaN500v1 Devices .................................53

Figure III.24: Extracted and Simulated $\mathrm{C}_{\mathrm{Eq}}$ versus $\mathrm{V}_{\mathrm{G}}$ for GaN150 Devices ....................................53

Figure III.25: Exctacted and Simulated $\mathrm{R}_{\mathrm{Eq}}$ versus $\mathrm{V}_{\mathrm{G}}$ at $2.5 \mathrm{GHz}$ for GaN500v1 ...........................55

Figure III.26: Extracted and Simulated $\mathrm{R}_{\mathrm{Eq}}$ versus $\mathrm{V}_{\mathrm{G}}$ at $2.5 \mathrm{GHz}$ for GaN150v0 ............................55

Figure III.27: Extracted $Q$ factor versus Frequency at $\mathrm{V}_{\mathrm{G}}=-3.5 \mathrm{~V}$ for GaN500v1 …........................57

Figure III.28: Extracted $Q$ factor versus Frequency at $\mathrm{V}_{\mathrm{G}}=-3.5 \mathrm{~V}$ for GaN150v0 …........................5 57

Figure III.29: Measured (lines) and Simulated (Dot) S11-parameter for W50L0.5 ............................60

Figure III.30: Measured (lines) and Simulated (Dot) S11-parameter for G W100L0.5 .......................60 
Figure III.31: Measured (lines) and Simulated (Dot) S11-parameter for G W200L0.5 . 61

Figure III.32: $\mathrm{C}_{\mathrm{Eq}}$ versus frequency for the W50L0.5 device at $\mathrm{VG}=-3 \mathrm{~V}$

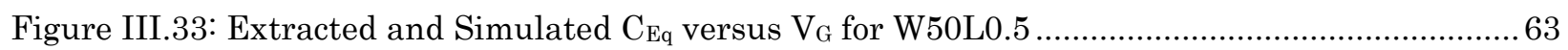

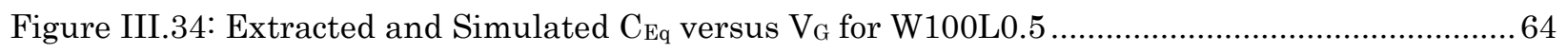

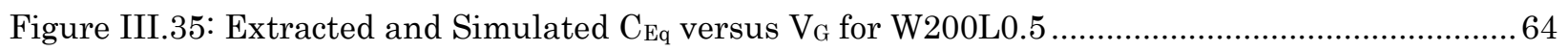

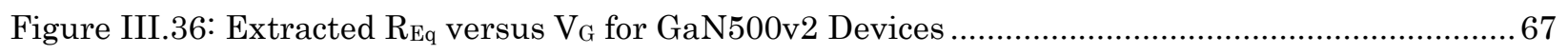

Figure III.37: Extracted (left) and Simulated (right) $C_{\text {Eq }}$ versus $V_{G}$ for various power levels [-10, 18]

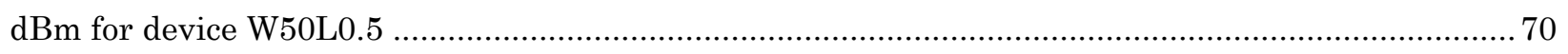

Figure III.38: Extracted (left) and Simulated (right) $\mathrm{C}_{\mathrm{Eq}}$ versus $\mathrm{V}_{\mathrm{G}}$ for various power levels [-10, 18]

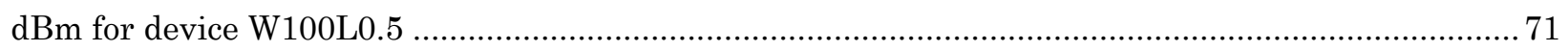

Figure III.39: Extracted (left) and Simulated (right) $\mathrm{C}_{\text {Eq }}$ versus $\mathrm{V}_{\mathrm{G}}$ for various power levels [-10, 18]

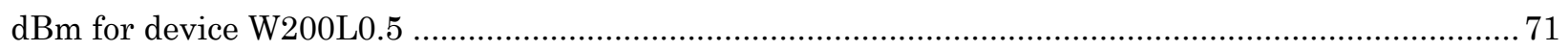

Figure III.40: Extracted $R_{\mathrm{Eq}}$ versus $\mathrm{V}_{\mathrm{G}}$ for various power levels $[-10,18] \mathrm{dBm}$ for device W50L0.5 (left), for W100L0.5 (middle) and for W200L0.5 (right) ......................................................................... 72

Figure III.41: Extracted Available (left) and reflected (right) RF Power versus Power from Source for

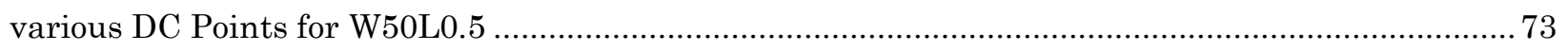

Figure III.42: Extracted Delivered RF Power versus Power from Source for various DC Points for W50L0.5 .73

Figure III.43: Simple Model for a HEMT in the switch configuration (a) ON-state and (b) OFF-state mode. .76

Figure III.44: Linear model used to model the switching GaN HEMT transistors (4x40) [[90] ..........80

Figure III.45: Equivalent Linear Circuit Model of GaAs FET Switch [91] ........................................ 81

Figure III.46: Schematic (left) of Common Gate Configuration standalone switch 2x200_GaN500v2 and Device in Microwave probes measurement setup (right) to Evaluate RF behavior ......................82 Figure III.47: Measured insertion loss of different transistor size in common gate, biased with $\mathrm{V}_{\mathrm{G}}=0 \mathrm{~V}$ (ON state) 83

Figure III.48: Measured Isolation of different transistor size in common gate, biased with $\mathrm{V}_{\mathrm{G}}=-5 \mathrm{~V}$ (OFF state)

Figure III.49: Measured Return Loss (S11) of different transistor size in common gate, biased with $\mathrm{V}_{\mathrm{G}}=0 \mathrm{~V}(\mathrm{ON}$ state $)$. .84

Figure III.50: Measured Return Loss (S11) of different transistor size in common gate, biased with

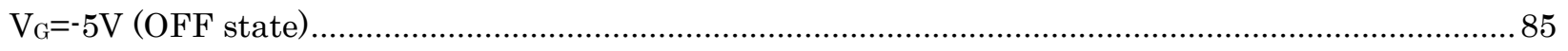

Figure III.51: Linear model used to model the switching GaN HEMT transistors. ............................ 86

Figure III.52: Measured and Modeled Insertion Loss and Return Loss (S11) of switch 2x25 biased with

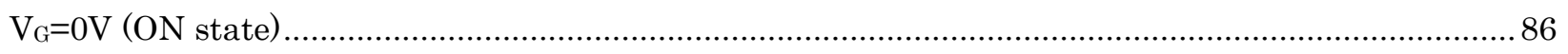


Figure III.53: Measured and Modeled Isolation and Return Loss (S11) of switch $2 \times 25$ biased with $\mathrm{V}_{\mathrm{G}}=-$ $5 \mathrm{~V}$ (OFF state) 87

Figure III.54: Schematic (left) and Layout (right) of the Proposed SPST Switch Structure .88

Figure III.55: Measured SPST performances 89

Figure III.56: Schematic (left) and Layout (right) of the Proposed SPDT Switch Structure .90

Figure III.57: Measured and Modeled Return Loss, Isolation, and Insertion Loss of SPDT Switch Structure .91

Figure IV.1: (left) Tri-band system with three fixed duplexers and (right) with a single tunable duplexer [96] .95

Figure IV.2: Simplified RF front-end for a typical beamforming system in a non-GaN technology [[30]

Figure IV.3: Example RF front-end possible with GaN with key components in blue [[30] .99

Figure IV.4: Photograph of a novel GaN HEMT based high power high-pass/low-pass phase shifter [99] 100

Figure IV.5: Measured insertion and return loss for high pass [99] ............................................. 101

Figure IV.6: Measured insertion and return loss for low pass [99] ................................................ 101

Figure IV.7: Measured phase difference between the two states [99] .............................................. 101

Figure IV.8: Microphotograph of the $0^{\circ} / 22.5^{\circ} \mathrm{GaN}$ switched-filter phase shifter [100]..................... 102

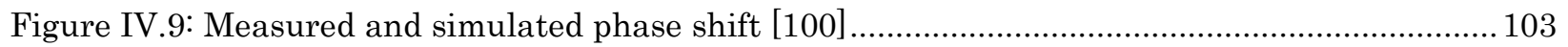

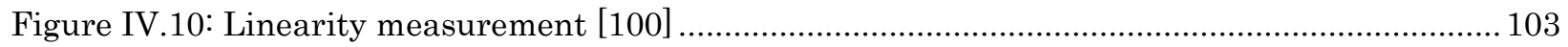

Figure IV.11: T-Phase shifter Circuit Configuration and microscope image [101] .......................... 104

Figure IV.12: Phase shift of the high-pass T-network and the corresponding inductance for $50 \Omega$

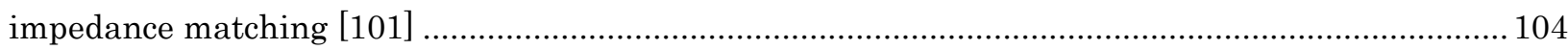

Figure IV.13: phase shift of the T-Phase shifter in the frequency range of $32 \mathrm{GHz}$ to $38 \mathrm{GHz}$ [101] 105

Figure IV.14: Phase shifter based on 3-dB/90。 branch-line hybrid coupler using distributed elements 107

Figure IV.15: Phase shifter based on 3-dB/90॰ branch-line hybrid coupler using lumped elements. 107

Figure IV.16: Layout of a Spiral Inductor Generated from NRC GaN150 PDK [105] ....................... 113

Figure IV.17: An Equivalent Circuit to Estimate LS, RS and Fres [105] ........................................113

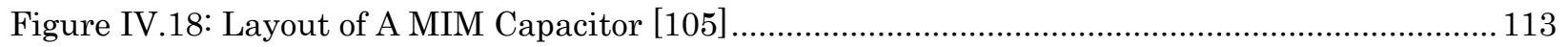

Figure IV.19: Layout of the designed hybrid coupler ...................................................................... 114

Figure IV.20: 3-D Layout view of the designed hybrid coupler …............................................... 115

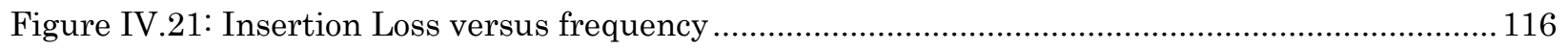

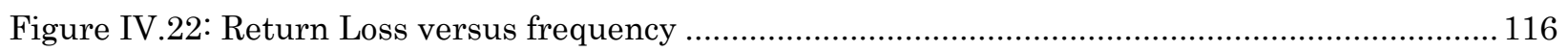

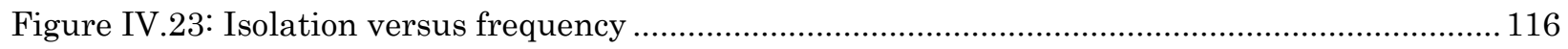




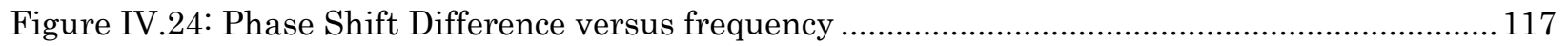

Figure IV.25: Layout of the designed Phase Shifter ............................................................................ 118

Figure IV.26: 3-D Layout view of the designed Phase Shifter ....................................................... 118

Figure IV.27: Return Loss at several DC Bias from -3.5 V to -5.5 V …….................................... 121

Figure IV.28: Insertion Loss of the Phase Shifter at several DC Bias from -3.5 V to -5.5 V..............121

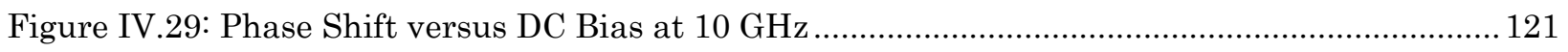

Figure IV.30: Layout of the designed hybrid coupler ..................................................................... 124

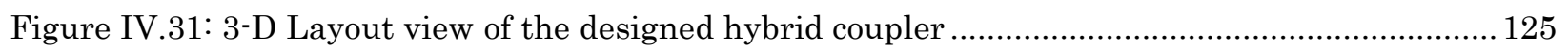

Figure IV.32: Return Loss at several DC Bias from -3.7 V to -4.8 V .............................................. 126

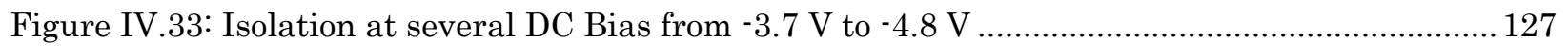

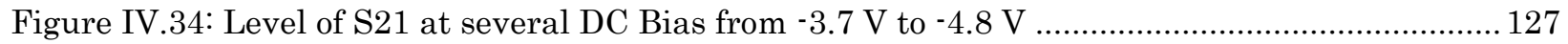

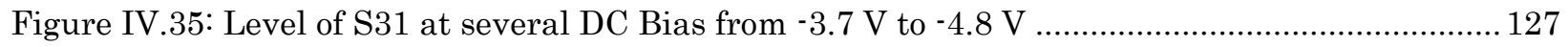

Figure IV.36: Phase Difference at several DC Bias from -3.7 V to -4.8 V......................................... 128

Figure IV.37: Block diagram of a feedback oscillator ....................................................................... 129

Figure IV.38: Photo of the CGHV1J025D GaN Transistor Die ....................................................... 134

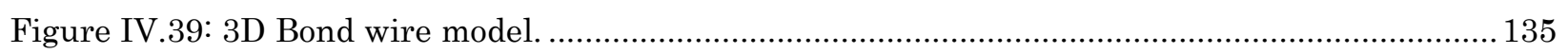

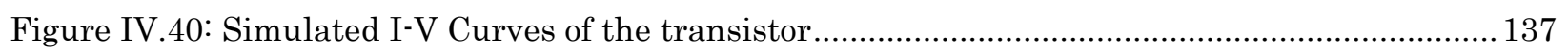

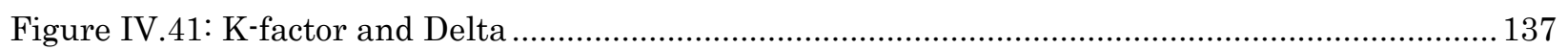

Figure IV.42: A typical distributed bias network with radial stub .................................................. 138

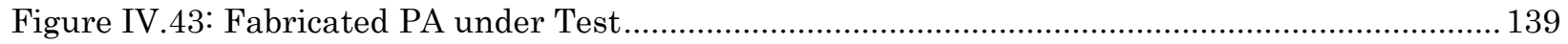

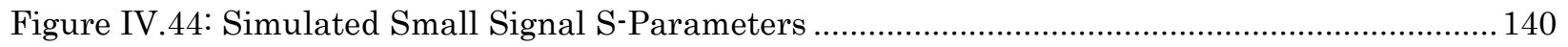

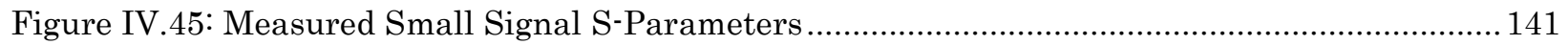

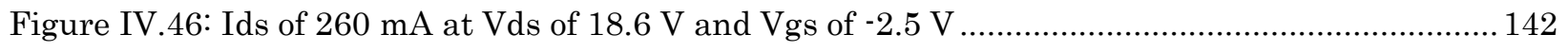

Figure IV.47: Fixed Frequency Oscillator Fabricated Prototype ................................................... 146

Figure IV.48: Small Signal Simulation Results (Open-Loop) ........................................................... 146

Figure IV.49: Harmonics Power Simulation of the FFO ................................................................. 146

Figure IV.50: Pure Voltage Sinusoid at the Output of the FFO ....................................................... 147

Figure IV.51: Pure Current Sinusoid at the Output of the FFO ..................................................... 147

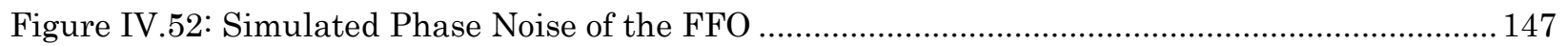

Figure IV.53: Harmonics Power Measurement of the FFO .......................................................... 148

Figure IV.54: Tunable Frequency Oscillator fabricated Prototype …................................................ 149

Figure IV.55: Oscillation frequency versus gate voltage …......................................................... 150

Figure IV.56: Output power versus gate voltage at fundamental frequency .................................... 151

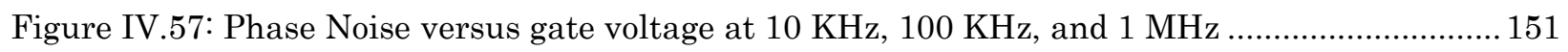

Figure IV.58: Printed antenna prototype with GaN varactor load. ................................................. 155 
Figure IV.59: Simulated return loss of the antenna alone 156

Figure IV.60 : Simulated antenna gain versus frequency ............................................................. 156

Figure IV.61 : Simulated normalized radiation pattern of the antenna at $11 \mathrm{GHz} . \ldots \ldots \ldots \ldots \ldots \ldots \ldots \ldots . . . . . . . . . . .157$

Figure IV.62: Simulated return loss of the loaded antenna for different reverse-bias voltages ........ 158

Figure IV.63: Measured return loss of the loaded antenna for different reverse-bias voltages......... 159

Figure IV.64 : Measured loaded antenna gain versus frequency for various DC bias....................... 160

Figure IV.65 : Measured radiation pattern in H-plane of the loaded antenna under various DC bias 160

Figure IV.66 : Measured radiation pattern in E-plane of the loaded antenna under various DC bias 161

Figure IV.67 : (left) patch antenna (right) slot feeding loaded with GaN varactor........................... 163

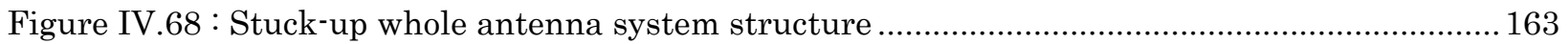

Figure IV.69 : Simulated return loss of the antenna alone .............................................................. 164

Figure IV.70 : Simulated antenna gain versus frequency …........................................................ 165

Figure IV.71 : Simulated normalized radiation pattern of the antenna at $11.35 \mathrm{GHz} . \ldots \ldots \ldots \ldots \ldots \ldots \ldots . . . . . . .165$

Figure IV.72: Simulated reflection coefficient (S11) of the loaded antenna for different varactor

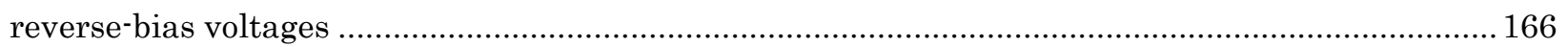

Figure IV.73: Measured return loss (S11) of the loaded antenna at no DC bias applied ................... 168

Figure IV.74 : Measured loaded antenna gain versus frequency at no bias DC applied ................... 169

Figure IV.75 : Measured normalized radiation pattern of the loaded antenna at 10.6 GHZ with no bias

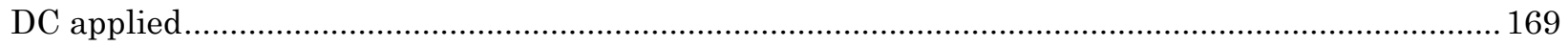

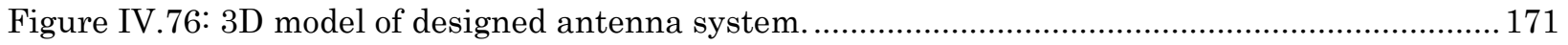

Figure IV.77: Fabricated prototype of the antenna ..................................................................... 172

Figure IV.78: The feeding system including the three SPDT switch structures ................................ 172

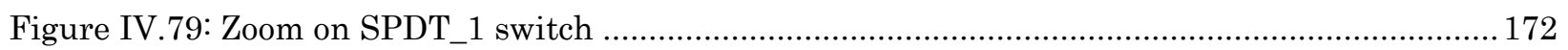

Figure IV.80: Zoom on the wires bonding connecting the SPDT_1 to The feeding system ................. 172

Figure IV.81: Front view of the fabricated antenna....................................................................... 174

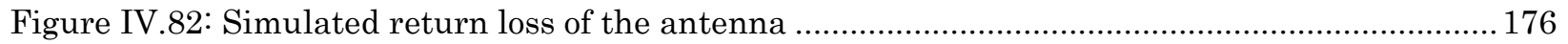

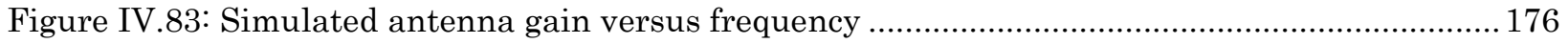

Figure IV.84: Simulated normalized H-plane at $2.43 \mathrm{GHz}$ for four cases mentioned in Table IV.4.. 177

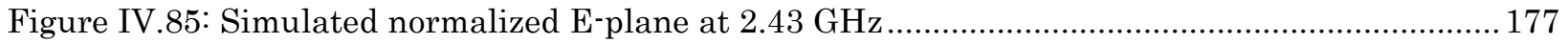

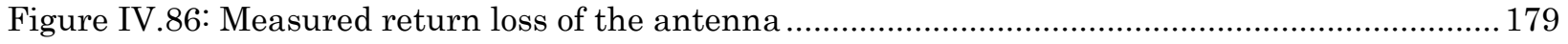

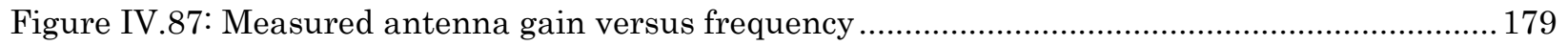

Figure IV.88: Measured normalized H plane at $2.43 \mathrm{GHz}$ for four cases mentioned in Table IV.4 .180 Figure IV.89: Measured normalized E plane at $2.43 \mathrm{GHz}$............................................................. 180 
Figure IV.90: Measured S-parameters for case_1 at Pin of $0 \mathrm{dBm}$. 182

Figure IV.91: Measured S-parameters for case_2 at Pin of $0 \mathrm{dBm}$ 182

Figure IV.92: Measured S-parameters for case_3 at Pin of $0 \mathrm{dBm}$ 183

Figure IV.93: Measured S-parameters for case_4 at Pin of $0 \mathrm{dBm}$ 183

Figure IV.94: Measured insertion loss versus input RF power for case_1 at $2.43 \mathrm{GHz}$ 184

\section{List of tables}

Table II.1: Comparison between properties of the most commonly used semiconductor technologies [1]

Table II.2: Parameters used in the selection of a grow substrate for GaN devices: lattice constant (a), thermal conductivity at $300 \mathrm{~K}\left(\mathrm{~K}_{300}\right)$, thermal expansion coefficient $(\mathrm{aL})$, wafer diameter and

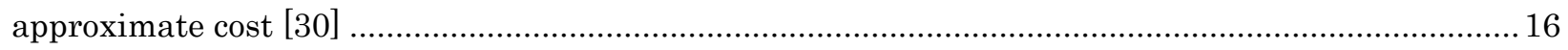

Table III.1: Presented GaN500v1 and GaN150v0 Devices Information........................................... 47

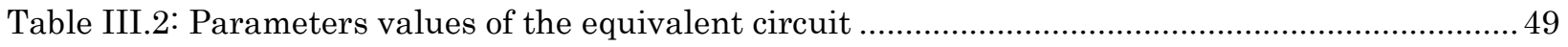

Table III.3: Comparison of capacitance performances between GaN500 and GaN150 ......................58

Table III.4 : Presented GaN500v2 Devices Information ....................................................................58

Table III.5: Parameters values of the equivalent circuit ................................................................59

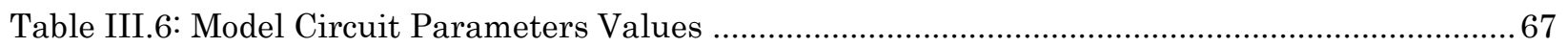

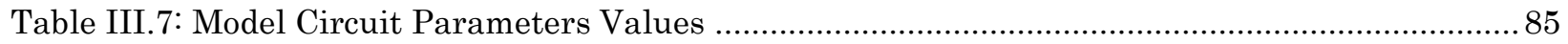

Table III.8: Performances of the SPDT at the Marker m4 ….......................................................... 91

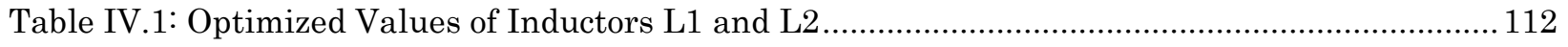

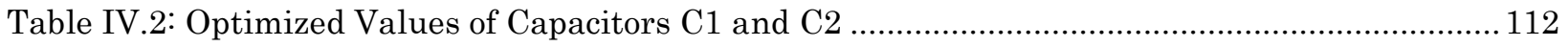

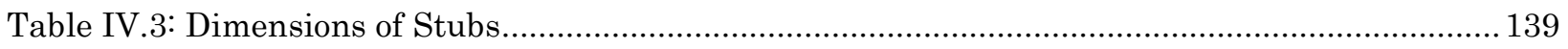

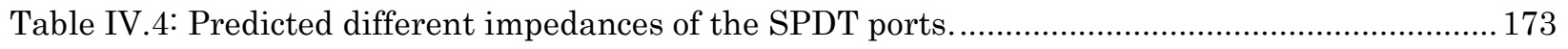

Table IV.5: Switching configuration to achieve radiation pattern reconfigurability......................... 174 


\section{List of Acronyms}

GaN Galium Nitride

AlGaN aluminum gallium nitride

GaAs Galium Arsenide

SiC silicon carbide

Si silicon

InP indium phosphide

SoP System-on-Package

FET Field-effect transistor

HEMT High Electron Mobility Transistors

2DEG two-dimensional electron gas

HBT Heterojunction bipolar transistor

HEMT High electron mobility transistor

HVPE Hydride vapour phase epitaxy

LNA Low־noise amplifier

PA Power Amplifier

MBE Molecular beam epitaxy

MEMS Micro-electromechanical systems

MIM Metal-insulator-metal

MMIC Monolithic microwave integrated circuit

MOCVD Metal-organic chemical vapour deposition

NRC National Research Council Canada

PCB Printed circuit board

RF Radiofrequency

SPDT Single-pole-double-throw

SPST Single-pole-single-throw

AIA Active Integrated Antennas 


\title{
Acknowledgements
}

\author{
الحمد له (Alhamdulillah)
}

Seldom anything is achieved without help, support, guidance, encouragement, love and care of other people. I am fortunate to have such people around me who have made my journey during my doctoral studies fruitful, rewarding and enjoyable.

Firstly, I am particularly indebted to my supervisors Pr. Mohammed HIMDI from University of Rennes 1 and Pr. Langis ROY from Carleton University, and my co-supervisor, Dr. Olivier LAFOND from University of Rennes 1, for the unconditional support, valuable guidance and endless patience they generously provided during my thesis research. I must also note the financial support of my advisors. I graciously thank Pr. Barry Syrett for accepting to be my co-supervisor at Carleton University for the last year. I am also grateful to Pr. Rony AMAYA and Pr. Garry TARR for their useful advice when I start dealing with GaN HEMT devices.

I am also grateful to Dr. Ibrahim HAROUN from NRC for giving me the opportunity to get the possibility of an internship in NRC and to work side by side with the NRC GaN group team. I have gained valuable insight into the GaN technology.

I graciously thank Pr. Steve McGARRY, Pr. Mustapha YAGOUB for accepting to be a part of my $\mathrm{PhD}$ thesis committee members.

I wish to express my sincere thanks to all staff of Laboratory IETR and the department of electronics at Carleton University, for providing me with all the necessary facilities for the research. Special thanks go to Blazenka POWER, Anna LEE and Mikhail NAGUI from Carleton University, and also Noelle LE BER from University of Rennes 1 for their previous helps. 
Also, special thanks are reserved for fellow colleagues Tyler Ross, Nathan Jess, Shiecheng YANG and Alexis MARTIN, who continue to set an example for graduate students with their thirst for knowledge and enthusiasm to share it.

On a personal note, I would like to express my sincerest gratitude to my brother Bouchaib and his wife, Bouchra, for their financial and moral support since I came to France for study. I would also like to express my deepest gratitude to my parents, brothers, and sisters (meaning also nieces) for the continuous encouragement which helped me endure tough moments. My gratitude also goes to my friends in Rennes and Ottawa, who encouraged me when needed. The name of best friends in particular should be highlighted. Tarik DANKIR for his encouragements and moral support and Imad ABDELJAOUAD who has been an inspiration and example for me since I first moved to Canada for my $\mathrm{PhD}$ degree.

Last but certainly not least, my future wife Imane EL ABBOUDY, whose love, understanding, and patience gave me the confidence and strength I needed to achieve success.

\section{Abdelaziz HAMDOUN}

October, 2016

O Allah, benefit me by that which You have taught me, and teach

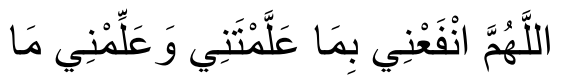
me that which will benefit me, and increase me in knowledge.

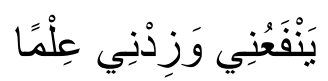




\section{Abstract}

This thesis demonstrates the feasibility of using gallium nitride $(\mathrm{GaN})$ technology in reconfigurable $\mathrm{RF}$ systems. GaN-based varactor diodes and switch circuits are pursued as promising candidates for high-power/high-frequency applications. The first part is devoted to active $\mathrm{GaN}$ device development. Active components were realized using the Canadian National Research Council (NRC) GaN HEMTs process. Based on three process, such as, GaN150v0 (gate length of 0.15um), GaN500v1 and GaN500v2 (both with gate length of 0.5um), many varactor diodes with size different have been manufactured and characterized via DC and RF smallsignal and large-signal measurements. Then, the varactor diodes were modeled by analytic equations containing empirical coefficients. These expressions have been introduced for the first time for the voltage dependency of equivalent capacitance $\left(\mathrm{C}_{\mathrm{Eq}}\right)$ and series resistance $\left(\mathrm{R}_{\mathrm{Eq}}\right)$ and can be used as a general model to represent the nonlinear behavior of $\mathrm{GaN}$ based varactors. For small-signal operation, all of the developed equations describing $\mathrm{R}_{\mathrm{Eq}}$ and $\mathrm{C}_{\mathrm{Eq}}$ are only bias voltage and device geometry dependent, while for large-signal operation, the influence of RF-power must be taken into account. In addition, different size single stand-alone switches were fabricated using GaN500v2. By analyzing the small-signal measurements, it was observed that the isolation is high at low frequencies but quickly drops with increasing frequency. Also, it was observed that the larger the device the lower the insertion loss and the poorer the isolation will be. Moreover, based on these small-signal measurements, a model was introduced.

The second part addresses the integration and design aspects of the reconfigurable proposed circuits, such as tunable phase shifter, reconfigurable $3-\mathrm{dB}$ $90^{\circ}$ hybrid coupler, tunable frequency oscillator, beam switching antenna array and matching reconfigurable patch antenna operating below $10 \mathrm{GHz}$. Here the developed GaN varactors and switches are used for achieving the tenability purpose. 


\section{Résumé en français}

Cette thèse a pour objectif de démontrer la faisabilité de l'utilisation de la technologie Nitrure de Gallium (GaN) dans les systèmes RF / micro-ondes reconfigurables. La technologie $\mathrm{GaN}$ doit permettre de supporter des puissances élevées avec un rendement important. Le GaN qui présente une large bande interdite possède des propriétés physiques lui permettant d'être largement utilisé pour la conception de circuits actifs à forte puissance. En effet, ses qualités intrinsèques, champs de claquage élevé, forte conductivité thermique, forte densité d'électrons, et bonne vitesse de saturation, lui confèrent de très bonnes performances aux hautes fréquences que ce soit pour les applications de puissance ou faible bruit. La technologie GaN est donc un candidat très prometteur pour la réalisation de fonctions hyperfréquence haute puissance/haute fréquence, telles que les amplificateurs de puissance, les mélangeurs, les oscillateurs contrôlés en tension (VCO) ou encore les amplificateurs faible bruit (LNA).

Cependant, malgré les caractéristiques intéressantes des dispositifs GaN, il reste encore des défis de fabrication à relever avant que cette technologie ne soit largement adoptée à l'avenir. Ces défis sont liés à deux aspects, le premier technologique et le second commercial. Technologiquement, les dispositifs HEMTs basés sur le GaN sont affectés par deux phénomènes importants, à savoir l'effet de piégeage et l'effet thermique (ou auto-échauffement des dispositifs) qui peuvent directement causer la dispersion du courant. Commercialement, un inconvénient de la technologie GaN concerne le coût de fabrication des composants. La réduction du coût de production est un facteur important pour pouvoir largement commercialiser les dispositifs GaN. De plus, le peu de disponibilité de matière GaN conduit à épitaxier le GaN sur des substrats tels que Silicium (Si), Carbure de Silicium (SiC) et le Saphir, ce qui peut dégrader les caractéristiques des composants GaN. Ces aspects sont abordés dans le chapitre II de ce manuscrit. 
Les travaux de thèse s'inscrivent dans une stratégie de développement des dispositifs GaN nécessaires à la conception de circuits RF reconfigurables. Les travaux sont divisés en deux parties principales. La première partie de ce travail de thèse est consacrée au développement et à la caractérisation des circuits actifs réalisés à base de la technologie GaN. Pour cela une investigation approfondie autour de diodes Varicap et de commutateurs a été menée et présentée. Les composants actifs ont été réalisés en utilisant le processus de fabrication GaN HEMTs offert par le Conseil National de Recherches du Canada (CNRC). Pour ce faire, trois processus GaN HEMT : le GaN150v0 avec une longueur de grille de 0,15 um, le GaN500v1 avec longueur de grille de 0,5 um et le GaN500v2, ont été investigués pour fabriquer des diodes varicap avec des tailles différentes. Ces diodes ont ensuite été caractérisées en $\mathrm{DC}$, en petits et grands signaux RF jusqu'à $20 \mathrm{GHz}$. Ici, les diodes varicap sont réalisées simplement en reliant le drain à la source, conduisant à réaliser une jonction Schottky qui peut être considérée comme « Planar Schottky Varactor Diode (PSVD) ». La tension $\mathrm{V}_{\mathrm{G}}$ de polarisation est alors appliquée entre le drain/source et la grille. En outre, ce type de diode ne nécessite aucun changement particulier des couches épitaxiales du procédé de fabrication HEMTs. Ensuite, les diodes varicap ont été modélisées en petit et grand signal par des équations analytiques. Ces expressions ont été introduites pour décrire la variation de la capacité équivalente $\left(\mathrm{C}_{\mathrm{Eq}}\right)$ et la résistance série $\left(\mathrm{R}_{\mathrm{Eq}}\right)$ en fonction la tension $\mathrm{DC}$ appliquée sur le composant. Ces équations peuvent être utilisées comme un modèle général pour représenter le comportement non-linéaire des diodes varicap basées sur la technologie GaN. Pour le modèle petit signal, les équations analytiques développées ne sont dépendantes que de la tension de polarisation appliquée sur le dispositif et de la géométrie de la diode, tandis que pour le modèle grand signal, l'influence de la puissance RF a aussi été prise en compte pour décrire le comportement de $R_{\mathrm{Eq}}$ et $\mathrm{C}_{\mathrm{Eq}}$. Pour étendre l'étude autour des composants GaN actifs utilisés dans les systèmes RF reconfigurables, plusieurs commutateurs avec différentes tailles configurés en grille commune ainsi que des structures de commutateurs tels que le SPST (Single Pole Single Throw) et 
le SPDT (Single Pole Double Throw) ont été fabriqués, en utilisant le processus de fabrication GaN500v2. En analysant les mesures en petit signal des commutateurs simples, on a pu observer que l'isolement est bon aux basses fréquences, mais se dégrade rapidement avec l'augmentation de celle-ci. En outre, on a pu conclure que plus le dispositif est grand, plus les pertes d'insertion s'améliorent, alors que l'isolation se dégrade. Donc un compromis entre perte d'insertion et isolation a été fait pour choisir les transistors individuels qui ont par la suite été utilisés pour la conception des structures de commutateurs plus complexes (SPST et SPDT). De plus, en se basant sur ces mesures petit signal, un modèle circuit a été introduit.

La deuxième partie de ces recherches de thèse aborde les aspects de l'intégration de ces dispositif actifs GaN (diodes varactor et commutateurs) et de la conception de circuits reconfigurables proposés, tels qu'un déphaseur reconfigurable, un coupleur $\left(3 \mathrm{~dB}, 90^{\circ}\right)$ reconfigurable, un oscillateur accordable en fréquence, ainsi qu'un réseau d'antennes à commutation de faisceau et une antenne unitaire accordable en fréquence. Initialement, un nouveau RUN technologique devait nous permettre de concevoir de nouveaux designs complètement intégrés en technologie GaN notamment le déphaseur et le coupleur $\left(3 \mathrm{~dB}, 90^{\circ}\right)$ reconfigurable en fréquence mais malheureusement ce RUN technologique n'ayant pas été assuré, il a donc été décidé d'utiliser les diodes varactors et les commutateurs préalablement fabriqués et testés sous pointe. Ceci a engendré des soucis puisque ces puces étaient de très petites dimensions et donc difficilement découpables puis intégrables dans les circuits imprimés tels que l'oscillateur ou les antennes. Toutefois, dans un souci de mener jusqu'au bout cette étude et de réaliser les circuits et antennes reconfigurables, de gros efforts ont été consentis afin de récupérer des diodes et commutateurs sur les wafers de base. Il a donc pu être possible de réaliser l'oscillateur et les deux types d'antennes reconfigurables. Si l'oscillateur contrôlé en tension et reconfigurable grâce à une diode Varicap préalablement fabriquée et caractérisée n'a pu aboutir à des résultats expérimentaux concluants, les prototypes d'antenne élémentaire reconfigurable en fréquence ou en diagramme à l'aide d'un réseau d'antennes 
commutable ont permis de démontrer la faisabilité de tels concepts en utilisant des composants actifs en technologie GaN. 



\section{Chapter I}

\section{Introduction}

\section{Research Motivations}

Nowadays, people are living in a society in which processing, flow and exchange of information are vital for their existence. Connectivity and mobility are two major issues in such society, which are related to the flow and exchange of information. On one hand, portable devices with internet provide connectivity which allows communication as well as access to information. On the other hand, wireless technologies bring mobility. People can move and still be able to communicate while having access to various kinds of information. This is the reason why radio frequency $(\mathrm{RF}) /$ microwave systems have evolved to integrated formats. This approach is called system-on-package ( $\mathrm{SoP}$ ), where many technologies are mixed in one package allowing increased simultaneous functionalities. Furthermore, the recent trend in $\mathrm{RF} /$ microwave technology is toward circuits that are highly miniaturized integrated 
and reliable, with low power consumption, and reduced cost. Monolithic Microwave Integrated Circuits (MMICs), based on semiconductor technology, are one of the keys to meeting the requirements of this technology mixing. Additionally, most MMICs currently in production, such as gallium arsenide (GaAs), silicon carbide ( $\mathrm{SiC}$ ), silicon ( $\mathrm{Si}$ ), indium phosphide ( $\mathrm{InP}$ ), etc, operate in the microwave and millimeter-wave $\left(\mathrm{mm}^{-\mathrm{w}}\right)$ range with modest breakdown voltages and limited power handling capabilities. But there are increasing applications in the $\mathrm{RF} /$ microwave spectrum where higher power/frequency is required.

Over the past decade, several changes have drawn more attention to $\mathrm{RF} /$ microwaves power devices. Increasing demands of high power handling capabilities and worries about capabilities of functioning in harsh environments have created a need for alternatives to traditional semiconductors technology. Due to its high critical breakdown field associated with wide band gap energy as well as its high saturated electron velocity, gallium nitride $(\mathrm{GaN})$ is a potential solution to satisfy these simultaneous requirements for $\mathrm{RF}$, microwave and $\mathrm{mm}^{-\mathrm{w}}$ transmitters for communications and RADAR [1]. On one hand, GaN-based high electron mobility transistors (HEMTs) power devices are considered as an excellent candidate for future power devices due to its high breakdown voltage and high saturation drain current [1]. On the other hand, besides being used for high power purposes, GaN HFETs devices are also a strong candidate to be used in harsh environment receiver applications due to the robust nature and good low noise performance of these devices [2], where others semiconductors can suffer.

With the advent of this wide band gap material, enormous progress has been made until now especially in the development of GaN technology. Consequently, more and more industries and academic research labs in the field of semiconductors have introduced different GaN HEMTs processes (i.e United Monolithic Semiconductors (UMS), TriQuint, Cree, NRC (National Research Council Canada), etc). These devices are intended to fulfill the growing demands for high power at high 
frequency electronic components as well as for high voltage power switches operating at higher frequencies. Furthermore, GaN HEMTs based MMIC technology is now challenging the other traditional semiconductor technologies such as GaAs mostly by offering the highest level of integration and the smallest form factor while keeping to operate with high voltage and to handle high power levels.

However, some challenges remain with GaN technology. The most serious problem is the cost of a $\mathrm{GaN}$ substrate in which the power devices are fabricated and the pace of maturation of their technological processes. Another issue is the lack of bulk GaN source material which leading to the need for GaN growth by using mismatched substrates such as $\mathrm{Si}, \mathrm{SiC}$ and sapphire.

The recent trend in $\mathrm{RF} /$ microwaves technology is toward circuits that are highly reconfigurable compacted and reliable with more functionalities, with low power consumption, and reduced cost. In particular, the downsizing demands in $\mathrm{RF} /$ microwaves wireless communication systems are growing exponentially as the number of multi-band and multi-service systems has increased along with the need for high power handling capabilities at higher frequency. The tunable and reconfigurability aspect of modern $\mathrm{RF} /$ microwaves front-ends transceivers ( $\mathrm{Tx} / \mathrm{Rx}$ ) is also an interesting subject concerning GaN HEMTs technology. Research into the usage of $\mathrm{GaN}$ based high power $\mathrm{RF}$ switches and varactors provide further opportunities for GaN to achieve these demands.

GaN technology is emerging as a major promising candidate for reconfigurable high power circuits for RF front-end Tx/Rx. Moreover, there is a wide consensus that the antenna system should be integrated in the same package with the front-end integrated circuit [3]. As an example, mobile communications (including SATCOM) imply that reconfigurable radiation pattern antennas are now of interest for such applications to steer the antenna beam. So antenna array system with a controlled gain and phase between single elements are often considered. In particular, for mobile applications, the antenna array must exhibit the capability to 
align its beam in the direction of the strongest signal. Hence, an antenna array with beam-steering capability is imperative and could be integrated on the same package with its two crucial components, phase shifter and gain amplifier to produce the desired amplitude and phase.

Fulfilling the in-depth development of GaN HEMTs technology for tunable high power circuits, it has become the motivation of this research to investigate novel highly compact reconfigurable active microwave circuits operating up to X-band frequency by using developed GaN varactor and switch models.

The main aim of this thesis research is to investigate and characterize GaN-based varactor diodes and switches that will be integrated to realize reconfigurable $\mathrm{RF} /$ microwave circuits operating up to $\mathrm{X}$-band frequency. This band of frequency from $8 \mathrm{GHz}$ to $12 \mathrm{GHz}$ is primarily used for military communications and Wideband Global SATCOM systems. It is mostly used in radar applications including continuous-wave, pulsed, single-polarization, dual-polarization, synthetic aperture radar and phased arrays. X-band radar frequency sub-bands are used in civil, military and government institutions for weather monitoring, air traffic control, maritime vessel traffic control, defense tracking and vehicle speed detection for law enforcement. This band is less susceptible to rain fade than the $\mathrm{Ku}$ Band due to the lower frequency range, resulting in a higher performance level under adverse weather conditions.

This thesis research has been conducted as a collaboration between University of Rennes 1 through "I'Institut d'Electronique et de Télécommunications de Rennes (IETR)", and Carleton University through the "Department of Electronics (DOE)". This collaboration is created from the $\mathrm{PhD}$ cotutelle (joint) program offered by both institutions. DOE has the expertise in CAD for electronic circuit design, $\mathrm{RF}$ and microwave circuits, photonics and integrated circuit using semiconductor technologies including GaN technology. A group of researchers from Carleton University is deeply involved in developing of circuits based on GaN technology. 
These researchers have a good relationship with the National Research Council (NRC) which provides GaN HEMTs foundry facilities, via CMC, and are involvedwith NRC's GaN team to develop this novel semiconductor technology. As a result, students as well as researchers from Carleton University can get access to this foundry. On the other hand, IETR is one of the largest and most research intensive labs in the design and characterization of active antennas up to millimeter-wave bands. Moreover, IETR provides facilities allowing the design of antennas, such as, EM simulators (i.e. CST, HFSS, etc.), unique measurement facilities in France including network analyrers up to $110 \mathrm{GHz}$ for S-parameter measurements, and an anechoic chamber working up to $110 \mathrm{GHz}$ for measuring radiation patterns of antennas.

\section{Objective of Research}

The proposed overall objective of this research is to study reconfigurable integrated microwave circuits using developed $\mathrm{GaN}$ varactor and switch models for use, for example, in X-band systems. It is expected that this project will demonstrate a new concept of realizing reconfigurable large-signal microwave circuits based on GaN technology, which will be used for the first time as active varactors and switches co-integrated in low cost microstrip technology to achieve the tunability of circuits.

The proposed specifics objectives are:

1. Explore possibilities of emerging GaN technology for integrated circuits that are miniaturized such as phase shifter and frequency reconfigurable hybrid couplers where the control functionality is achieved by using varactor diodes.

2. Investigate possibilities for $\mathrm{RF}$ reconfigurable circuits using active $\mathrm{GaN}$ components with a low-cost substrate. For this a reconfigurable oscillator, a beam switching antenna array and a matching reconfigurable patch antenna operating below $10 \mathrm{GHz}$ were designed. 
3. Demonstrate a working prototype that combines active GaN devices and passive components so as to experimentally validate the proposed concepts.

It will be shown that the developed GaN varactor and switch models can be used along with passive circuit designs in low cost microstrip technology so as to achieve, in the same circuit, reconfigurability and good RF performance. Additionally, this research project will demonstrate the great potential of GaN for use in other tunable microwave high power applications such as oscillators and reconfigurable antennas.

\section{Thesis Organisation}

The thesis begins with the chapter II dealing with a review of gallium nitride technology. Then, chapter III presents the characterization and modeling of gallium nitride varactor and switch devices manufactured with NRC process. The final chapter IV covers contributions and realized applications over this thesis research. 


\section{Chapter II}

\section{A Review of the Literature of Gallium Nitride Technology}

\section{Gallium Nitride Technology}

\section{Overview}

Since gallium nitride $(\mathrm{GaN})$ is used as a semiconductor technology to design the circuits for this thesis, it is necessary to review the characteristics of this technology and make a brief comparison with commonly used semiconductor technologies. GaN technology has been and still getting more and more attention for $\mathrm{RF} /$ microwave systems that require high power handling capabilities and low noise figure. This sets the field of GaN electronics in motion, and today the technology is improving the performance of several applications including, mobile communication, 
wireless base stations, communication and military radar systems, where its focus is shifting to further advantages such as high efficiency as well as the reduction of worldwide energy consumption [4].

Historically, development of semiconductor technologies based on Monolithic Microwave Integrated Circuit (MMIC) has always advanced to more and more performance improvements in terms of more high power handling capabilities, more gain, high efficiency, high linearity, decreasing noise figure [2]-[5]. In the early 1990s, GaN was revealed as a potential solution to satisfy the simultaneous requirements of high frequency and power [1]. GaN material has the capability of satisfying system device requirements from low-frequencies [7]-[8] through millimeter-waves [9]-[10]-[11]-[12] and was deemed an excellent material for being useful in high power applications. Because of its special properties, GaN technology is truly recognized as a revolutionary wide band gap technology resulting in dramatic afforded power levels that exceed many times ( $>5$ times) those levels achieved with other traditional technologies such as, indium phosphide (InP), gallium arsenide ( $\mathrm{GaAs}$ ), and silicon ( $\mathrm{Si}$ ). As a result, current densities in excess of $2 \mathrm{~A} / \mathrm{mm}$ have been reported [13]-[14]. High power densities of $40 \mathrm{~W} / \mathrm{mm}$ have also been reported at $4 \mathrm{GHz}$ with $1.2 \mathrm{~A} / \mathrm{mm}$ by Cree using field-plated devices [15]. Watt-level output power has been demonstrated and reported at W-band in [9]-[10]-[11] by HRL, Raytheon and Fujitsu. In [12] Cree has demonstrated power density higher than $25 \mathrm{~W} / \mathrm{mm}$ of gate periphery with unity current gain frequencies, Ft, greater than $40 \mathrm{GHz}$ reported. As a brief comparison, Figure II.1 recapitulates the most used semiconductor technologies and their limits in terms of power handling capability and operating frequency. 


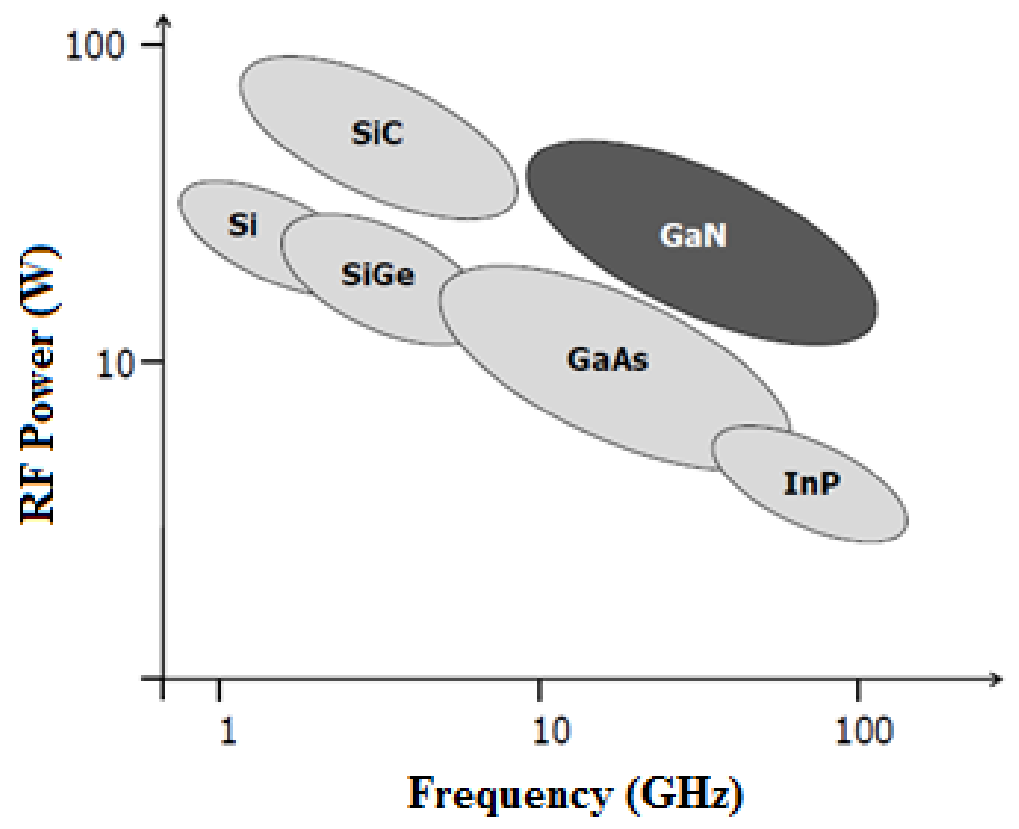

Figure II.1: Most used Semiconductor Technologies and their Frequency/Power limits [6]

GaN offers very high breakdown field, high electron mobility, and saturation velocity. It is also known that GaN has an excellent thermal conductivity and ability to operate at high temperatures which make it an ideal candidate for highpower and high-temperature microwave applications [1]. Moreover, GaN is a great candidate to enable the development of next generation of wireless and satellite communication systems that are needed to meet emerging demands for high data throughput, energy efficient wireless systems, reduced carbon footprints of wireless networks, high power electronic solutions for smart and efficient applications, and interoperability among diverse communication standards. Today, gallium nitride high electron mobility transistors (HEMTs) or heterojunction FETs (HFETs) are the most advanced electronic nitride devices. The hetero-structures offer many advantages such as high breakdown and transport properties of un-doped GaN. 
As shown in Table II.1, a comparison between the electrical/physical properties of the commonly used semiconductor technologies is illustrated. GaN has high sheet carrier density and channel charge density, which produce high output power. Also it has high electron mobility, which is largely responsible for low onresistance, and therefore, high power added efficiency could be achieved at higher frequencies. As a result, GaN HEMTs devices can achieve much higher breakdown voltage than the other technologies, very high current density, and sustain very high

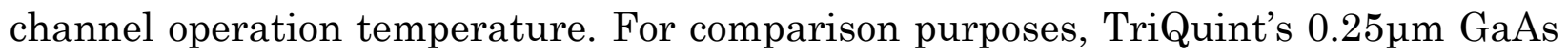
process has a typical breakdown voltage of $15 \mathrm{~V}$ [16] while TriQuint's $0.25 \mu \mathrm{m}$ GaN process has a typical breakdown voltage of $100 \mathrm{~V}$ [17]. Additionally, the combination of significant electron mobility and a good saturation velocity enable high speed operation of active devices. As a matter of fact, GaN circuits demonstrate their capabilities of operating adequately at high frequencies.

All of these presented factors indirectly improve the efficiency of GaN devices. Furthermore, possible epitaxial growth on SiC substrate, which has excellent thermal properties, makes this device optimal to operate at high power. Later in this chapter, the possibility of growing GaN over other substrates will be more detailed.

Table II.1: Comparison between properties of the most commonly used semiconductor technologies [1]

\begin{tabular}{|c|c|c|c|c|c|c|}
\hline \multicolumn{2}{|c|}{ Semiconductor (Typical Materials) } & $\mathrm{Si}$ & $\mathrm{GaAs}$ & $\mathrm{InP}$ & $\mathrm{SiC}$ & $\mathrm{GaN}$ \\
\hline Characteristic & Unit & & & & & \\
\hline Bandgap & $\mathrm{eV}$ & $\mathbf{1 . 1}$ & $\mathbf{1 . 4 2}$ & $\mathbf{1 . 3 5}$ & $\mathbf{3 . 2 5}$ & $\mathbf{3 . 4 9}$ \\
\hline Electronic Mobility at 300 $\mathrm{K}$ & $\mathrm{cm}^{2} / \mathrm{V} . \mathrm{s}$ & $\mathbf{1 5 0 0}$ & $\mathbf{8 5 0 0}$ & $\mathbf{5 4 0 0}$ & $\mathbf{7 0 0}$ & $\mathbf{1 0 0 0 - 2 0 0 0}$ \\
\hline Saturated Electronic Velocity & $\mathrm{x} 10^{7} \mathrm{~cm} / \mathrm{s}$ & $\mathbf{1}$ & $\mathbf{1 . 3}$ & $\mathbf{1}$ & $\mathbf{2}$ & $\mathbf{2 . 5}$ \\
\hline Critical Breakdown Field & $\mathrm{MV} / \mathrm{cm}$ & $\mathbf{0 . 3}$ & $\mathbf{0 . 4}$ & $\mathbf{0 . 5}$ & $\mathbf{3}$ & $\mathbf{3 . 3}$ \\
\hline Thermal Conductivity & $\mathrm{w} / \mathrm{cm} . \mathrm{k}$ & $\mathbf{1 . 5}$ & $\mathbf{0 . 5}$ & $\mathbf{0 . 7}$ & $\mathbf{4 . 5}$ & $>\mathbf{1 . 5}$ \\
\hline Charge channel Density & $\mathrm{x} 10^{13} / \mathrm{cm}^{2}$ & $\mathbf{0 . 3}$ & $\mathbf{0 . 2}$ & & & 1 \\
\hline Resistivity & $\Omega . \mathrm{cm}$ & $\mathbf{1 . 5}$ & $\mathbf{1 \times 1 0 ^ { 7 }}$ & & $\mathbf{1 \times 1 0 ^ { 5 }}$ & \\
\hline Relative Dielectric Constant & $\varepsilon_{\mathrm{r}}$ & 11.5 & 12.8 & 12.5 & 10 & $\mathbf{9}$ \\
\hline Loss tangent (at 10 GHz) & $\mathrm{Tg}$ & $\mathbf{0 . 0 1 5}$ & $\mathbf{0 . 0 0 0 4}$ & & $\mathbf{0 . 0 0 3}$ & \\
\hline
\end{tabular}


However, despite the superior characteristics of GaN device, there still remain some issues at multiple levels with respect to fabrication (process, device, circuit and system) that must be overcome before being widely adopted in future. These challenges that are impacting the development of GaN technology can be summarized in two main challenges such as technological (device processing) and commercial tasks.

The first technological (device processing) challenge is that the GaN HEMTs are affected by two important phenomena, namely the trapping effect and thermal effect (or self-heating), which can directly cause current dispersion such as collapse and rolling off as shown in Figure II.2. This existence, of dispersion effects observed in GaN devices, acts to restrict the drain current and voltage excursions, thereby limiting the initial expectations of device's performances in term of high power and high frequency.

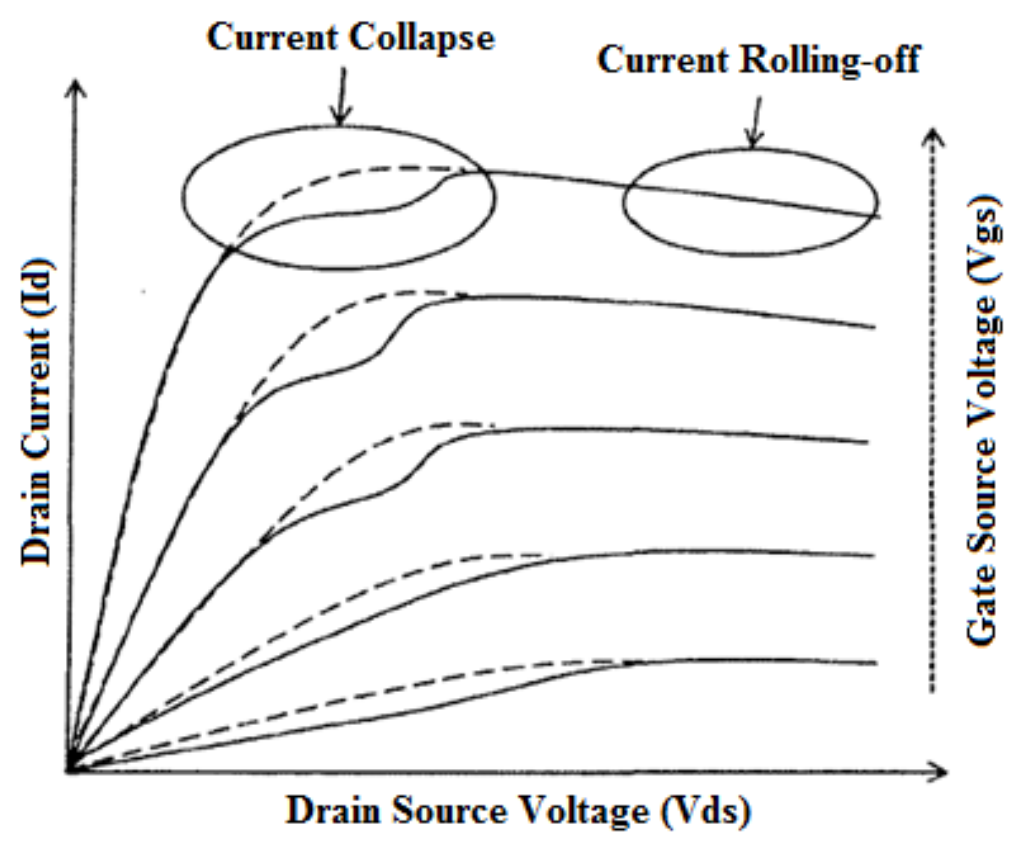

Figure II.2: Current due to trapping effect and thermal effect [18] 
Current collapse is explained as a transient and recoverable reduction in drain current response caused by trapping effects. In general, this phenomena means that some electrons are captured by traps somewhere in AlGaN/GaN HEMTs heterostructures [18] as shown in Figure II.3. These captured electrons reduce the sheet electron density in the channel, leading to increase dynamic $R_{\text {on }}$ leading to drain current dispersion [19]. In principle, electron trapping can occur at different locations such as metal/AlGaN interface, the un-gated AlGaN surface near the gate edge, AlGaN/GaN interface, and the buffer GaN layer during HEMT operation.

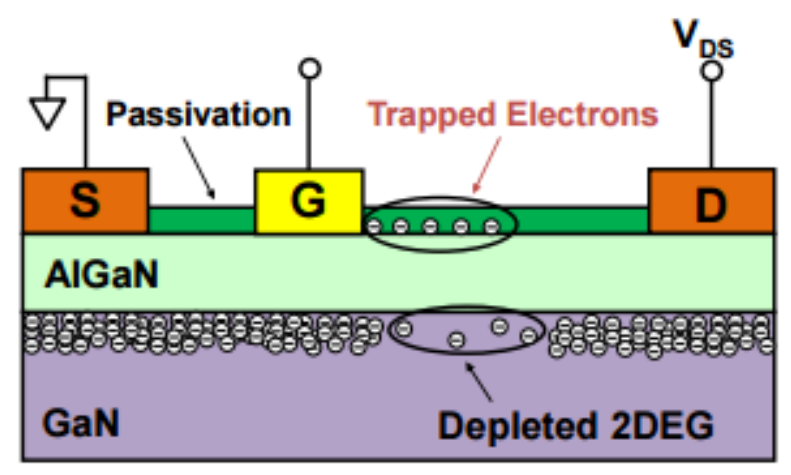

Figure II.3: A sketch of an AlGaN/GaN HEMT structure with trapped electrons [19]

Most reported works, explaining this effect, conclude that trapping effect can mainly reside at the surface [20]-[21], in the AlGaN barrier layer, at the twodimensional electron gas (2DEG) interface, and in the GaN buffer layer [22]-[23]. This effect is produced when a large bias voltage is applied between the source and drain, which induces a quick acceleration of the electrons in the conducting channel. These fast random moving electrons can be injected into an adjacent region of the device structure and became trapped if this region contains a significant concentration of traps which is the case for the GaN buffer layer.

On the other hand, thermal and self-heating effects exist in every power semiconductor device. Especially when the device is working in the high voltage and high power region, the self-heating causes electron carriers to accelerate in random directions instead of following the drain to source channel. Some of the electrons eject 
themselves out of the channel, causing significant roll off in the high voltage and high current region, which is observed in DC current variations, as shown in Figure II.2.

The simplified vertical semiconductor structure of a GaN/AlGaN HEMTs is illustrated Figure II.4.

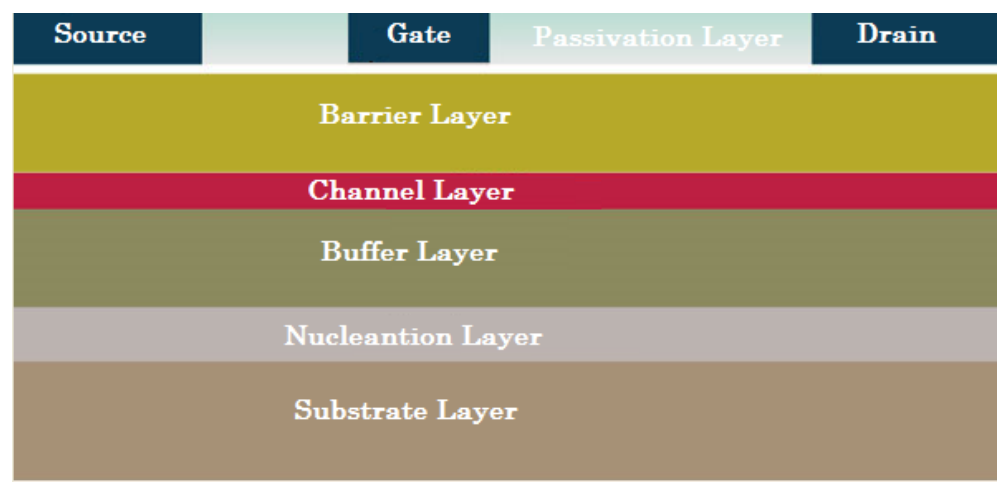

Figure II.4: Cross-Section of HEMTs (Not Drawn to Scale)

- Barrier: This semiconductor layer isolates the gate and the channel so that very low current flows between the channel and the gate. In GaN FETs, the barrier is typically made of aluminum gallium nitride (AlGaN).

- Channel: The channel or 2-DEG is the region where electrons flow from the source to the drain. The channel is typically made of high-quality GaN.

- Buffer: The role of the buffer is to restrict the movement of the electrons within the channel. In other words, the buffer acts as a barrier that prevents the electrons from wandering into the substrate. In a GaN FET, the buffer is typically made of $\mathrm{GaN}$ doped with carbon $(\mathrm{C})$ or iron $(\mathrm{Fe})$.

- Nucleation: The growth of GaN structures on a foreign substrate requires the careful matching of the GaN lattice to this substrate, involving the engineering of suitable nucleation layers between the substrate and the buffer layer.

- Substrate: The substrate provides mechanical support, heat spreading, and electromagnetic confinement. GaN FETs use a foreign substrate (that is, Sapphire, Si or ( $\mathrm{SiC})$, but not GaN). Because the substrate is not GaN, it has a different crystal lattice than the buffer. This creates dislocations in the 
material and ultimately reduces the electrical isolation between gate and channel.

Even though there have been remarkable improvements in growth and device fabrication using GaN material, trapping effects is still a challenge and cannot be eliminated perfectly, which still an active key research topic in AlGaN/GaN HEMT applications. Additionally, an accurate HEMT model including trapping effects is still needed for development of implementation of AlGaN/GaN HEMT-based circuits.

The second aspect of GaN HEMTs that is a significant disadvantage is the cost of fabrication. Reducing the production cost is an important factor for power GaN HEMTs commercialization. This big commercial challenge is due to a general lack of availability. There are more and more companies over the world develop GaN devices, so it is possible to buy some GaN HEMTs from distributors today, but the selection is limited and still expensive compared to others technologies. Additionally, the lack of device standardization between these distributors means that there are no real second sources for any of the devices on the market today. No major manufacturer is going to use a GaN transistor in a high-volume application until interchangeable devices are available from multiple manufacturers. Furthermore, the unavailability of bulk GaN source material led to the need for GaN growth on mismatched substrates such as $\mathrm{Si}, \mathrm{SiC}$ and sapphire, this also poses an issue which leads to poor quality epitaxial films with dislocation densities.

\section{Choice of the Substrate}

Choosing an appropriate substrate to grow GaN material is still an issue for developing GaN circuits. Beside Si or GaAs circuits for example, a Si or GaAs substrate is normally used; while GaN circuits have to be grown over another substrate because it is difficult to manufacture a GaN substrate due to the lack of the material. Since GaN is not easy found in nature, it must be grown on another 
substrate, typically $\mathrm{Si}, \mathrm{SiC}$ or Sapphire. There are three techniques to grow GaN over a mismatched substrate. These techniques have been employed widely in the past to reduce the high dislocation density and for the realization of GaN substrate with large size and high quality. One of the common methods is done by using metal organic chemical vapour deposition (MOCVD), after which the substrate is removed [24]. The success of this technique is resided in reducing dislocation density [24]; however it leads to selective area epitaxy due in large part to mechanical stresses and mechanical deformations, thus resulting on a limitation of GaN wafer. Typically GaN wafer are limited to two or three inches in diameter and thicknesses of about $100 \mu \mathrm{m}$. A second way to grow GaN, which is currently the most commonly used technique, is by hydrid vapour phase epitaxy (HVPE) [25] which is initially used in the growth of GaAs and InP. This is faster and has lower defect density than MOCVD (5x104/cm ${ }^{2}$ for HVPE, compared to $8 \times 10^{7} / \mathrm{cm}^{2}$ for MOCVD). Additionally, it has been demonstrated that larger wafer sizes can be realized [26]. A third method for growing GaN is by ammonothermal growth [27]. This technique offers even better defect densities $\left(1 \times 10^{3} / \mathrm{cm}^{2}\right)$. However, growth of the crystal is much slower: $0.4-16 \mu \mathrm{m} / \mathrm{h}$ [28], compared to 100-500 $\mu \mathrm{m} / \mathrm{h}$ for HVPE [29].

While GaN substrate is still unused, the decision of choosing a foreign substrate is crucial and based on several factors such as lattice constant, thermal conductivity, thermal expansion coefficient, wafer size and, of course, cost. The lattice parameter and the coefficient of thermal conductivity are the two crucial important properties that should closely be matched between the GaN and the mismatched substrate. Any mismatch between these two properties can result in defects in the film such as misfit and threading dislocations due to lattice mismatch and cracking or bowing due to the thermal mismatch. Table II.2 summarizes these parameters that are used when a substrate is selected. 
Table II.2: Parameters used in the selection of a substrate for GaN devices: lattice constant $(\alpha)$, thermal conductivity at $300 K\left(K_{300}\right)$, thermal expansion coefficient $(\alpha L)$, wafer diameter and approximate cost [30]

\begin{tabular}{|c|c|c|c|c|c|}
\hline Material & 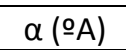 & $\mathrm{k}_{300}$ (W/K.m) & $\alpha \mathrm{L} 5.59(\mathrm{ppm} / \mathrm{K})$ & Diameter (inches) & Cost \\
\hline $\mathrm{GaN}$ & 3.189 & 130 & 5.59 & $\leq 4$ & High \\
\hline $\mathrm{SiC}$ & 3.08 & 490 & 4.2 & $\leq 4$ & Medium \\
\hline Sapphire & 2.747 & 41 & 7.5 & $\leq 6$ & Low \\
\hline $\mathrm{Si}$ & 5.4301 & 148 & 3.59 & $\leq 12$ & Low \\
\hline AiN & 3.112 & 285 & 4.2 & $\leq 2$ & High \\
\hline Diamond & 3.75 & 2000 & 1.18 & $\leq 4$ & High \\
\hline
\end{tabular}

In the process used for designing the circuits for this thesis, a $\mathrm{SiC}$ substrate is used. $\mathrm{SiC}$ substrate provides a good compromise between cost, excellent thermal properties which makes this device optimal to operate at high power.

\section{NRC GaN500 MMIC HEMT Process Features and Limitations}

The great interest raised by AlGaN/GaN HEMT devices in the international semiconductor scientific community in general, for high power/frequency applications, has resulted in that an important number of industries and academics labs research (i.e United Monolithic Semiconductors (UMS), Fraunhofer IAF, Ferdinand Braun Institute (FBH), TriQuint, CREE, National Research Council Canada (NRC), etc) in the world are nowadays fully involved to demonstrate new microwave HEMTs devices in this new GaN technology. The presented varactor and switch circuits in this thesis were manufactured by the Canadian Photonics Fabrication Center (CPFC) at the National Research Council Canada (NRC).

NRC offers a foundry services for AlGaN/GaN HEMT-based MMIC process for both university and industry customers. At the time of this thesis research, the technology features include a metal gate of $0.5 \mu \mathrm{m}$ in the GaN500 process which was commercialized. Also, a metal gate of $0.15 \mu \mathrm{m}$ in the GaN150 process was still under 
development and months later its release was announced. Recently, the GaN500 process has been stopped to be commercialized in meanwhile the GaN150 process has just been released. Some varactors have been fabricated using the initial version (non-commercial) of this process when it was still under development. The access availability to the NRC GaN process is possible only through a company called CMC Microsystems which is an intermediate between NRC and costumers.

The proposed foundry [31], combines high power density, excellent thermal conductivity, and high gain performance. The HEMT devices are fabricated on an aluminum gallium nitride/gallium nitride $(\mathrm{AlGaN} / \mathrm{GaN})$ hetero-structure grown on semi-insulating $4 \mathrm{H}-\mathrm{SiC}$ substrates. Semi-insulating $\mathrm{SiC}$ is one of the most attractive substrate materials for electronic applications, because of the favorable combination of lattice mismatch, isolation, and thermal conductivity [[18]. In addition, AlGaN/GaN HEMT structures are formed by using ammonia molecular beam epitaxy (MBE) in a SVTA nitride MBE system and are consisted of a $3 \mathrm{~nm}$ thick aluminum nitride (AlN) nucleation layer, a $1.1 \mu \mathrm{m}$ thick carbon-doped GaN insulating buffer, followed by a $0.2 \mu \mathrm{m}$ thick undoped GaN layer, and then $1.5 \mathrm{~nm}$ AlN spacer plus $20 \mathrm{~nm}$ AlGaN barrier [32]. Currently both standard processes, GaN500 and GaN150, use 3-inch diameter $\mathrm{SiC}$ wafers of $75 \mu \mathrm{m}$ thickness. More details about the wafer growth can be found in [32]-[33].

As shown in Figure II.5, where the complete process sequence is given, the first step in the GaN500 process fabrication as well as in GaN150 process is to deposit the Ohmic contact using the Ohmic mask (OHMIC Layer). The contacts are then annealed at a high temperature to ensure that a low-resistance contact is made to the two-dimensional electron gas (2DEG), where the carriers are located. The next step is to define the active area of the devices using the mesa mask (MESA Layer), and then it is isolated by etching it down to the buffer layer.

Gate metals are then formed by depositing a passivation dielectric layer in the channel area and etching a slot for the gate contact. The gate contact metal 
(GATEMETAL layer) is deposited and a second dielectric encapsulation is applied. Openings are etched into the dielectric (VIA1 layer), where required, to allow connection to the ohmic and gate contacts.

Then, a thin layer of nichrome (NICHROME Layer) is deposited where resistors are required. The first level of interconnect metal ( $1 \mu \mathrm{m}$ of gold) is next deposited using the $1 \mathrm{ME}$ mask (1ME Layer). The $1 \mathrm{ME}$ provides contacts to the ohmic metal and gate pads, as well as the nichrome layer. This layer is also used to form a field plate, which is connected to the source and overlaps the gate.

Next, the $1 \mathrm{ME}$ layer is isolated with a dielectric deposition and via openings are defined (VIA2 Layer) where required to define the vias. This layer of dielectric is used as the insulator in the MIM capacitors.

Finally, the areas where a second level of interconnect is defined (2ME Layer) to bridge over other layers is defined with a temporary layer of a special resist using the bridge mask (BRIDGE Layer). The $2 \mathrm{ME}$ layer is usually $1 \mu \mathrm{m}$ of gold and is used to make the top layer of the MIM capacitors.

These process steps and Figure II.5 are reproduced with permission of the NRC. 


\section{Deposit and anneal OHMIC contacts, etch MESA, deposit passivation}

Etch gate slot, Deposit gate

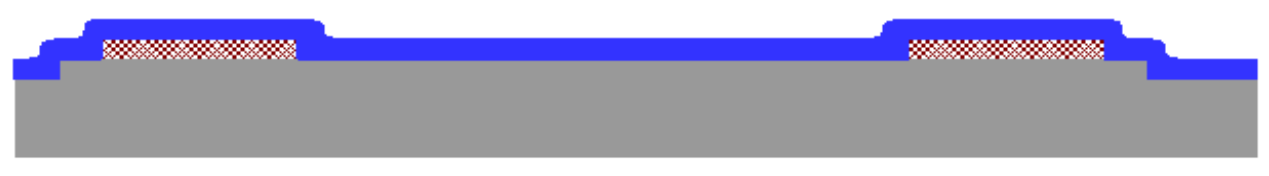

Deposit isolation dielectric, etch VIA1

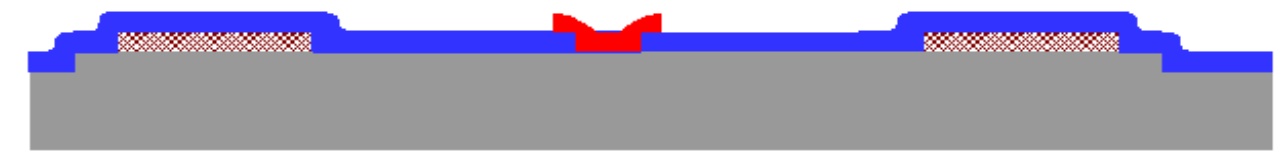

Deposit NICHROME (not shown), deposit 1ME

Deposit second dielectric, etch VIA2
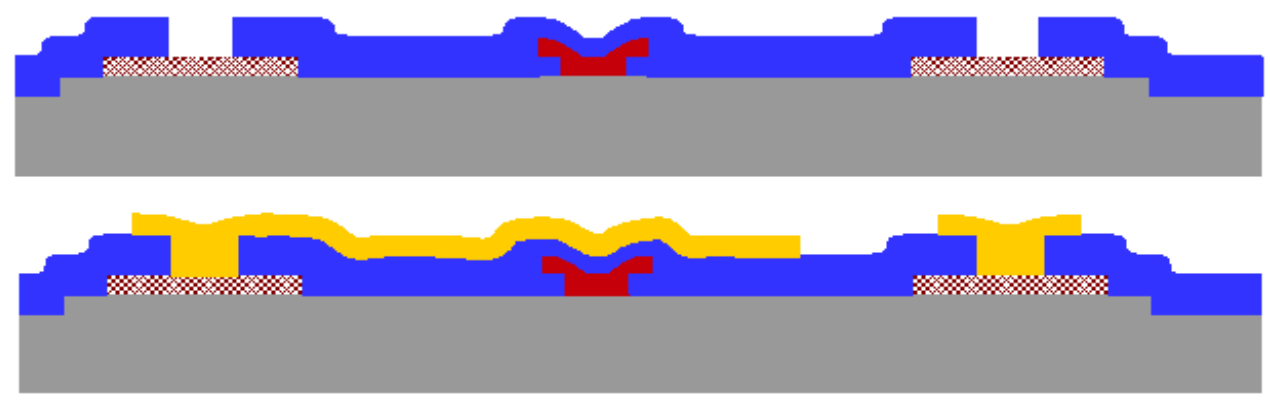

Pattern wit sacrificial BRIDGE resist
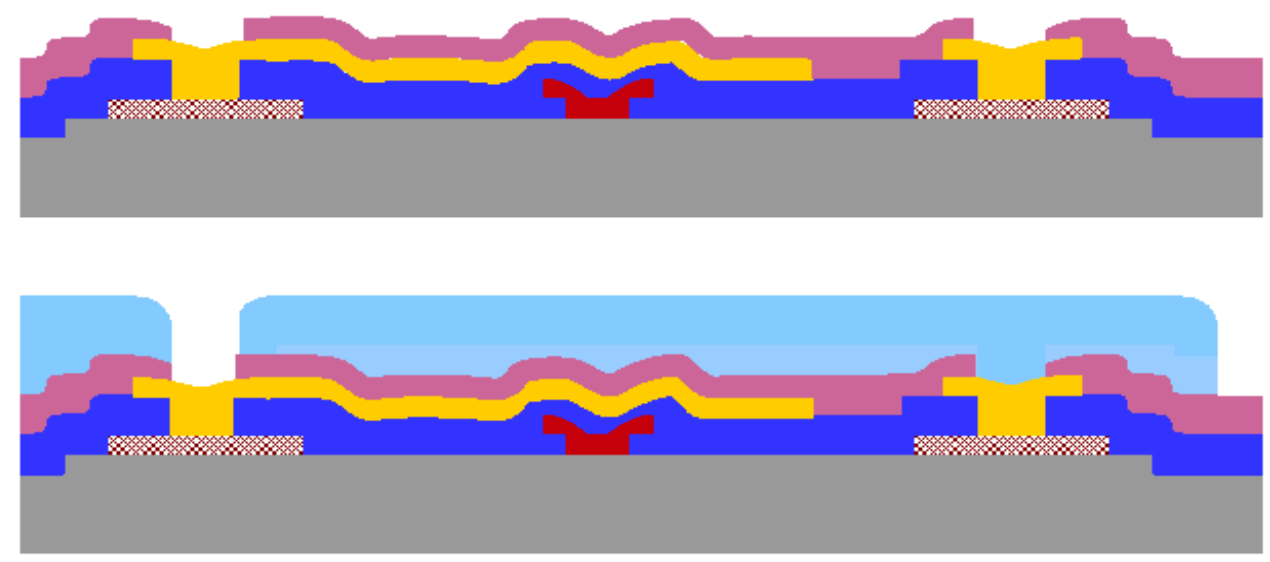

Deposit 2ME, Remove sacrificial resist

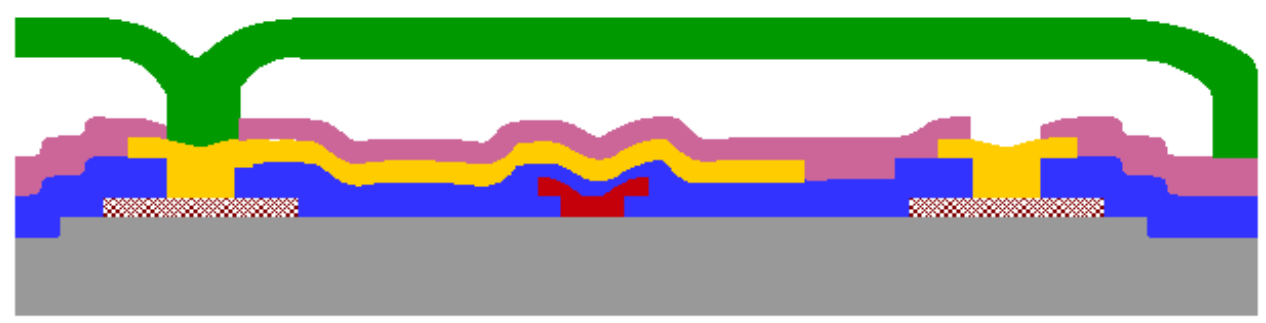

Figure II.5: Cross-sectional view of HFET, showing process sequence (not to scale)

$$
\text { [31] }
$$


Moreover the two processes involve two layers of $1 \mu \mathrm{m}$ and $3 \mu \mathrm{m}$ gold, respectively, $1 \mathrm{ME}$ and $2 \mathrm{ME}$ for interconnect, and the second layer may be used to form either 100nm metal-insulator-metal (MIM) capacitors of $0.19 \mathrm{fF} / \mu \mathrm{m} 2$ or $1 \mu \mathrm{m}$ air-bridges. Thin-film nichrome resistors with sheet resistance of $50 \Omega / s q$ are also available. Spiral inductors in this process typically have a measured quality factor of about 12 to 18 and are wound a central empty area, with a recommended minimum size of $50 \times 50 \mu \mathrm{m}$. While capacitors with measured quality factors of at least 50 at $\mathrm{X}^{-}$ band are attainable.

GaN500v2 offers transistors with a unity current gain frequency, Ft, of 13 $\mathrm{GHz}$ and a maximum oscillation frequency, Fmax, of $60 \mathrm{GHz}$, and it is suitable for 30V maximum drain voltage bias, and yields power levels of $\sim 7 \mathrm{~W} / \mathrm{mm}$ (measured at 8 GHz). While GaN150v1 offers transistors with Ft of $35 \mathrm{GHz}$ and a Fmax of $75 \mathrm{GHz}$. Furthermore, GaN500v2 HFETs devices exhibit breakdown voltages (measured with channel pinched-off) generally greater than $200 \mathrm{~V}$, however a maximum drain voltage of $40 \mathrm{~V}$ is currently recommended. While a breakdown voltage VDS_BD (VDs measured at channel pinched-off) greater than $100 \mathrm{~V}$ and a maximum drain bias voltage of $30 \mathrm{~V}$ is currently recommended for GaN150v1 devices. Finally, the gate source voltage can operate from $-8 \mathrm{~V}$ to $2 \mathrm{~V}$, with at least a threshold voltage of $-4.6 \mathrm{~V}$ needed to turn these devices ON [31]. Additional details about the technology are provided in NRC's GaN design manual process [31].

As is traditionally known, as a part of any technology fabrication process, proper and accurate models are usually set and provided in the process design kit (PDK) to achieve any circuit's functionality. In the used GaN fabrication process, there is still work to be done as it lacks essential components desired for reconfigurable applications, such as the lack of varactor models, and also the lack of common gate $(\mathrm{CG})$ configured switch models, which are vital for revealing switch operation capabilities of these FETs. Moreover, the current version of the PDK only supports a limited number of active device sizes. This thesis will concentrate on 
proposing equivalent circuit models, firstly, for varactor diodes and then for switch in common gate configuration. Equivalent circuit models analysis for varactor diodes will be conducted in small signal and large signal while only small signal analysis will be conducted for switch devices. These developed models will be used in RF designs to verify them and validate their abilities of use in some RF applications.

\section{Use of $\mathrm{GaN}$ in Reconfigurable RF/Microwave Systems}

Monolithic RF/microwaves integrated active reconfigurable systems are necessary for applications which require lightweight, low cost, high reliability, ease of integration, and high volume. The recent development of high electron mobility transistor (HEMT) makes it possible to realize a high level reconfigurable $\mathrm{RF} /$ microwaves design, such as power amplifiers, phase shifters, low noise oscillators, and reconfigurable antenna array designs. The high efficiency power amplifier and the phase shifting constitute the important parts of a $\mathrm{RF} /$ microwaves transmitter block.

Due to the mature development of GaAs technology, beam-former circuitry could be integrated into the same package as the antenna array system [34]. There have been intensive developments of these listed components, which have relied on high performance semiconductor technologies especially GaAs HEMT technology. In order to achieve higher RF output power with higher efficiency, GaN technology is a very promising candidate for realizing designs, such as high efficiency tunable circuits, highly reconfigurable low noise power oscillators and integrated active beam forming transmit antenna arrays [35]. The current studies of this technology reveal that GaN has the potential to be the technology of choice for the next generation of transmitter/receiver ( $\mathrm{Tx} / \mathrm{Rx})$ modules where high power/frequency applications [36][37] and several functionalities on the same chip [38] are required. 
Active reconfigurable circuitry for transmitting and/or receiving $(\mathrm{Tx} / \mathrm{Rx})$ modes has been widely used for many applications, such as space and defense. In addition, high efficiency compact $\mathrm{Tx} / \mathrm{Rx}$ modules are increasly needed in $\mathrm{RF} /$ microwaves applications. Their development is driving the enhancement of HEMT MMIC technology, now extending to the promising GaN technology. Indeed, the trend in the last generation of $\mathrm{Tx} / \mathrm{Rx}$ module systems is to reduce the number of MMICs in the module by increasing their power handling capabilities and efficiencies using GaN [39]-[40]. The current state of art shows that GaAs technology has been intensively used for these systems at many frequency bands [41]-[42]. Meanwhile for GaN technology, many reconfigurable power amplifiers for $\mathrm{Tx} / \mathrm{Rx}$ modules have been reported in the last ten years. There has been some work on Tx/Rx module using GaN fox X-band [43], where three types of amplifiers (DA, HPA and LNA) were integrated on multi-layer low temperature co-fired ceramic (LTCC) substrates. In Figure II.6 the completely assembled front-end is shown. Output power levels of $6.8 \mathrm{~W}(38 \mathrm{dBm})$ for the driver amplifier (DA) and 20W (43 dBm) for the high power amplifier (HPA). Measured results are depicted in Figure II.7 and Figure II.8. The measured noise figure of the low noise amplifier (LNA) is in the range of $1.5 \mathrm{~dB}$ as shown in Figure II.9.

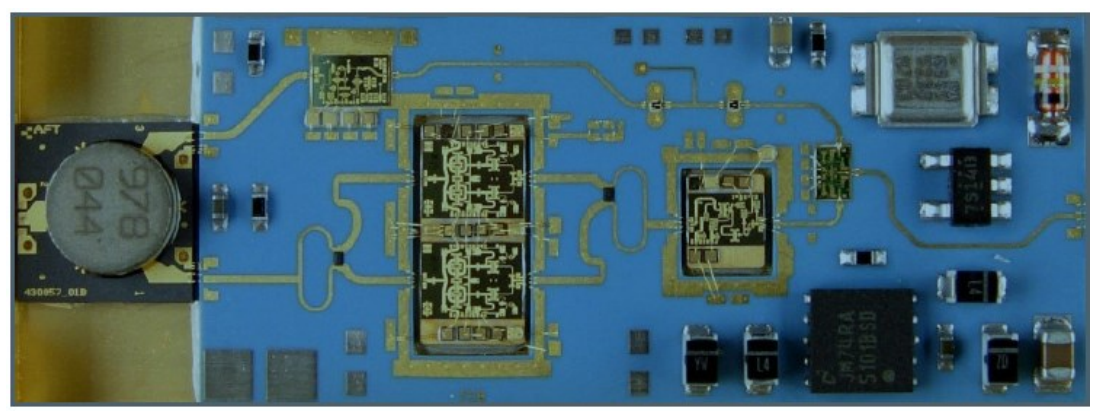

Figure II.6: Photo of a T/R-Module front-end with GaN MMIC chips [43] 


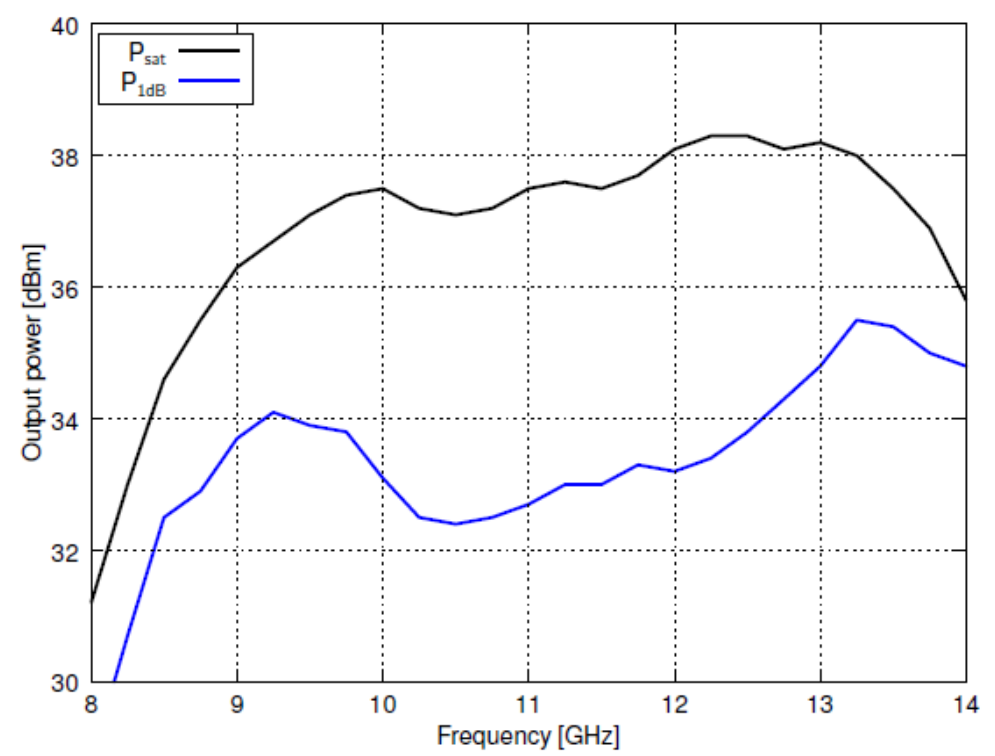

Figure II.7: Measured output power versus frequency of the GaN MMIC DA at $1 \mathrm{~dB}$ gain compression and at saturation with $\mathrm{Vds}=30 \mathrm{~V}$ and $\mathrm{Vgs}=-4.9 \mathrm{~V}$ [43]

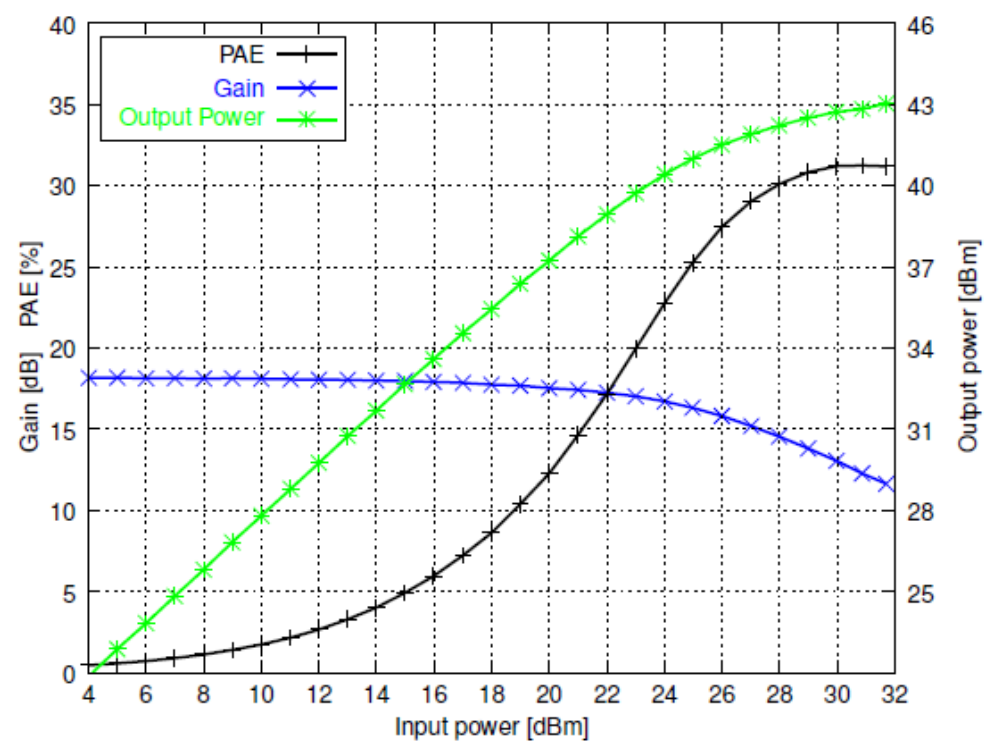

Figure II.8: Measured power performance of the GaN MMIC HPA at $11 \mathrm{GHz}$ with Vds $=30 \mathrm{~V}$ and $V g_{S}=-5 \mathrm{~V} . P \max =43 \mathrm{dBm} P A E \max =31 \%[43]$ 


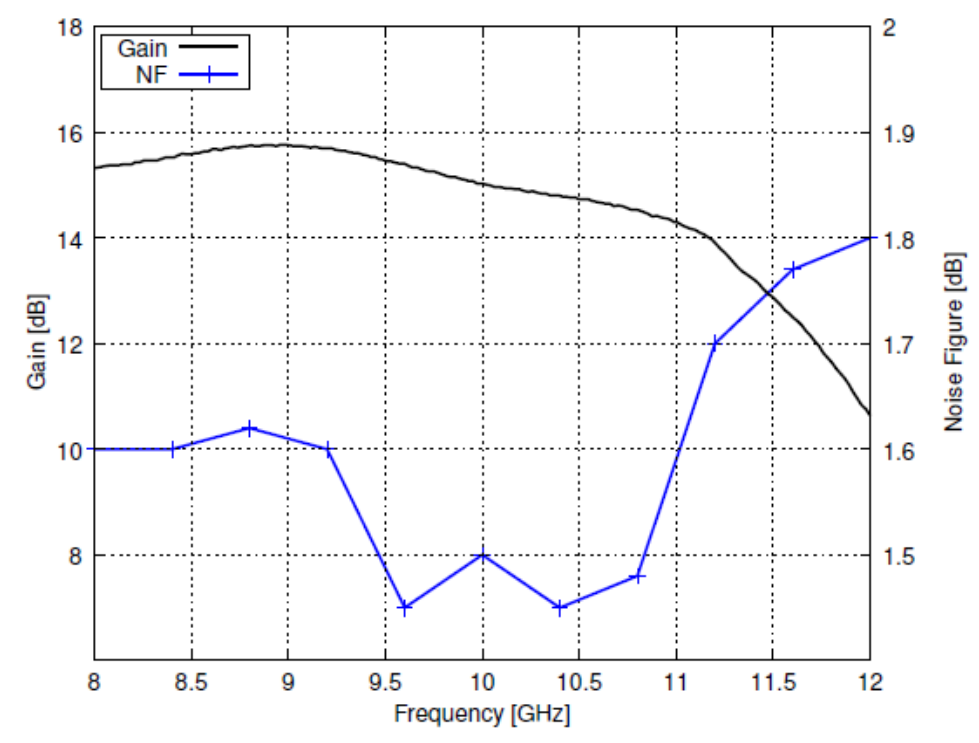

Figure II.9: Measured gain and noise figure versus frequency of the GaN MMIC

$$
L N A \text { with } V d s=15 \mathrm{~V} \text { and } V g_{S}=-3: 5 \mathrm{~V} . \mathrm{NFmin}=1: 45 \mathrm{dBm} \text { [43] }
$$

Another Tx/Rx module using GaN was reported [44], which contains a GaN power amplifier (PA) with output power higher than $10 \mathrm{~W}$ over $6-18 \mathrm{GHz}$ and a GaN low-noise amplifier (LNA) with a gain of $15.9 \mathrm{~dB}$ over 3.2-20.4 GHz. Figure II.10 shows the fabricated T/R module, while the Figure II.11 and Figure II.12 illustrate, respectively, the measured Pout of fabricated T/R a function of frequency and the measured S-parameters of GaN LNA MMIC.
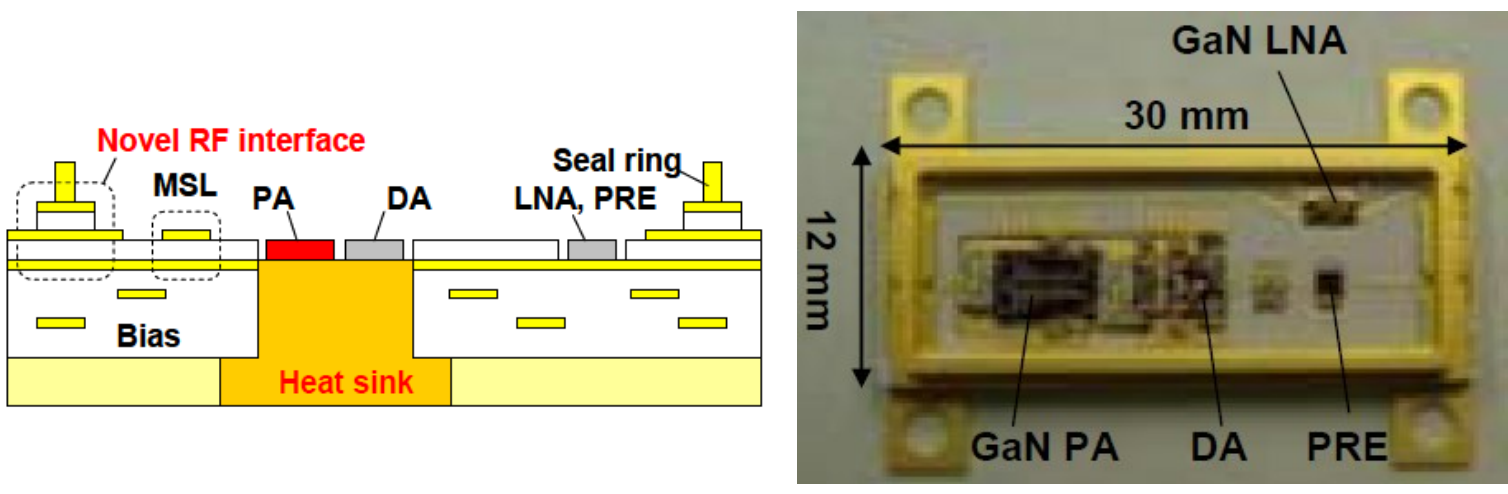

Figure II.10: (Left) A schematic view and (Right) a photograph of the Fabricated T/R module using HTCC technology [44] 


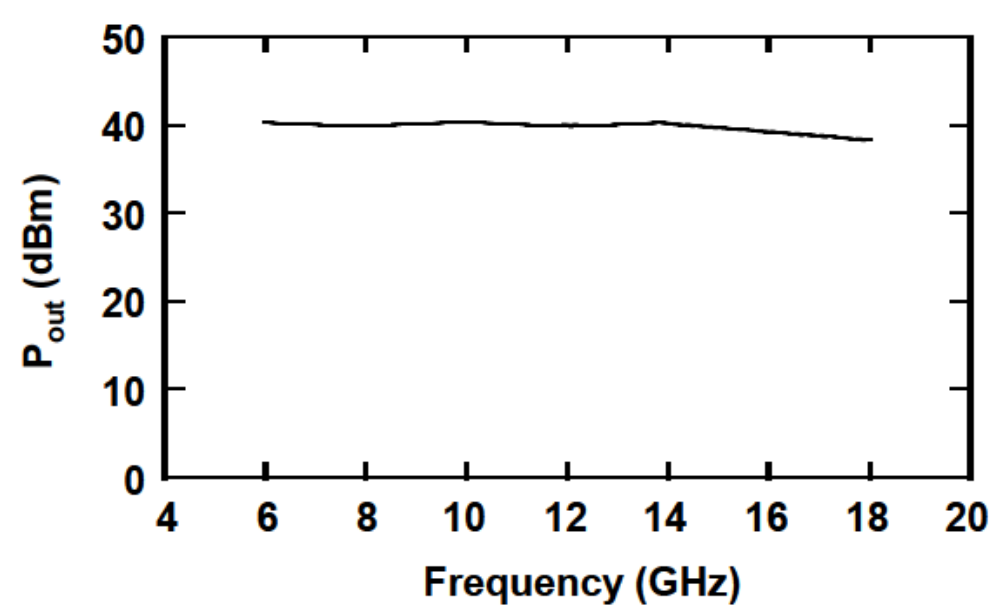

Figure II.11: Measured Pout of fabricated T/R module as a function of frequency, biased at $30 \mathrm{~V}[44]$

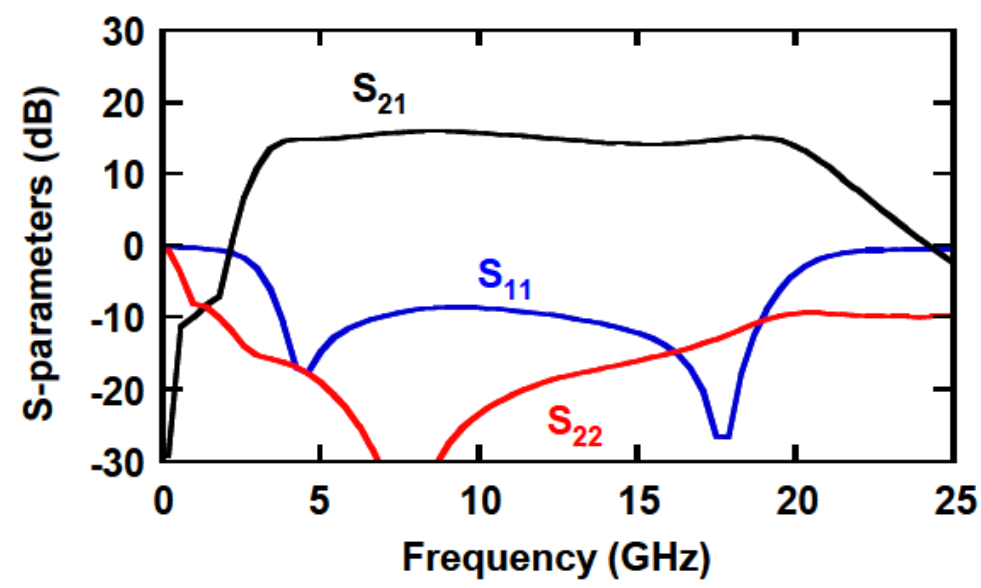

Figure II.12: Measured S-parameters of GaN LNA MMIC (Vds = 15 V) [44]

Using GaN technology, some implemented reconfigurable PAs have been reported. A reconfigurable Doherty PA has been presented in [45] based on a GaN MMIC modified Doherty PA with large bandwidth and reconfigurable efficiency. Photograph of the fabricated amplifier is illustrated in Figure II.13. The total size measures $2.9 \times 2.9 \mathrm{~mm}^{2}$. This design presents a new theory that makes it possible to analyze how the performance of the amplifier depends on the input network properties and the choice of gate bias voltage. The design achieved a power added efficiency (PAE) more than $31 \%$ at $9 \mathrm{~dB}$ output power back-off over a band from 5.8 
GHz to $8.8 \mathrm{GHz}$. The measured PAE is plotted versus output power in Figure II.14. The measured gain is plotted versus output power in Figure II.15. As shown, a smallsignal gain (Pout $=18 \mathrm{dBm}$ ) is larger than $7.4 \mathrm{~dB}$ up to $8.8 \mathrm{GHz}$ is achieved, whereas the gain at high output power $(\approx 33 \mathrm{dBm})$ stays within $8.5-9.0 \mathrm{~dB}$ for all frequencies.

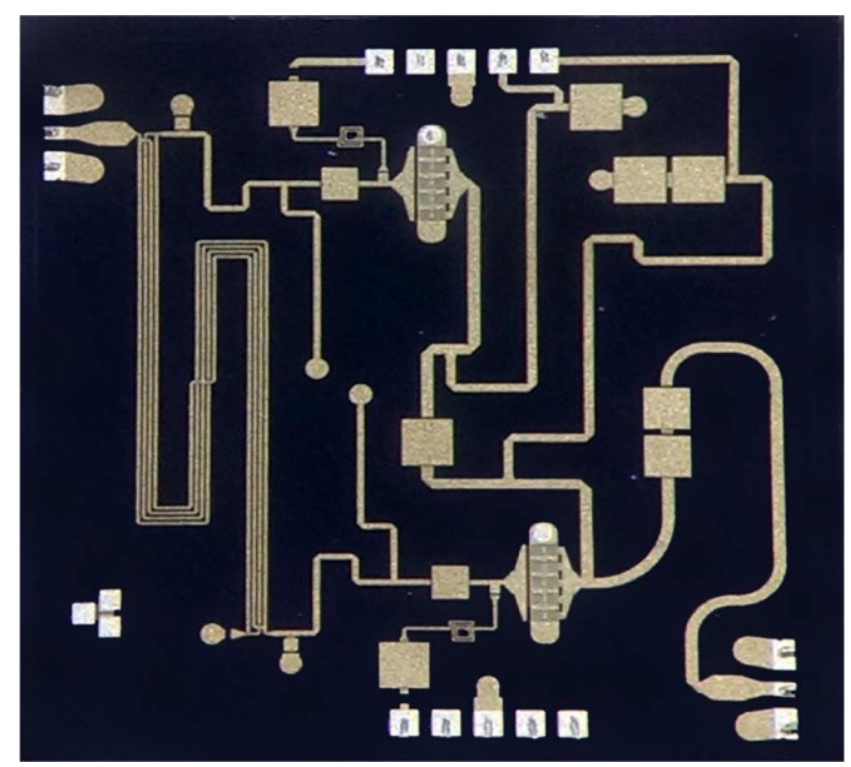

Figure II.13: Photograph of the fabricated amplifier [45]

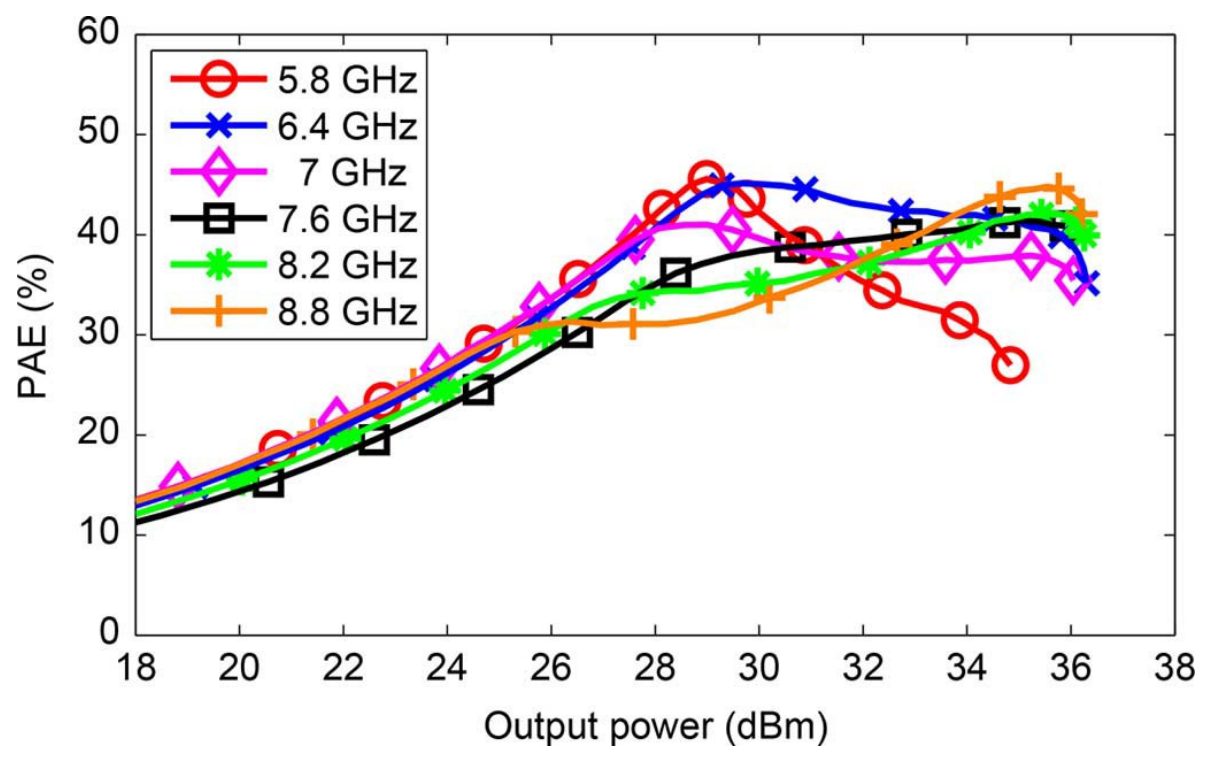

Figure II.14: Measured PAE versus output power [45] 


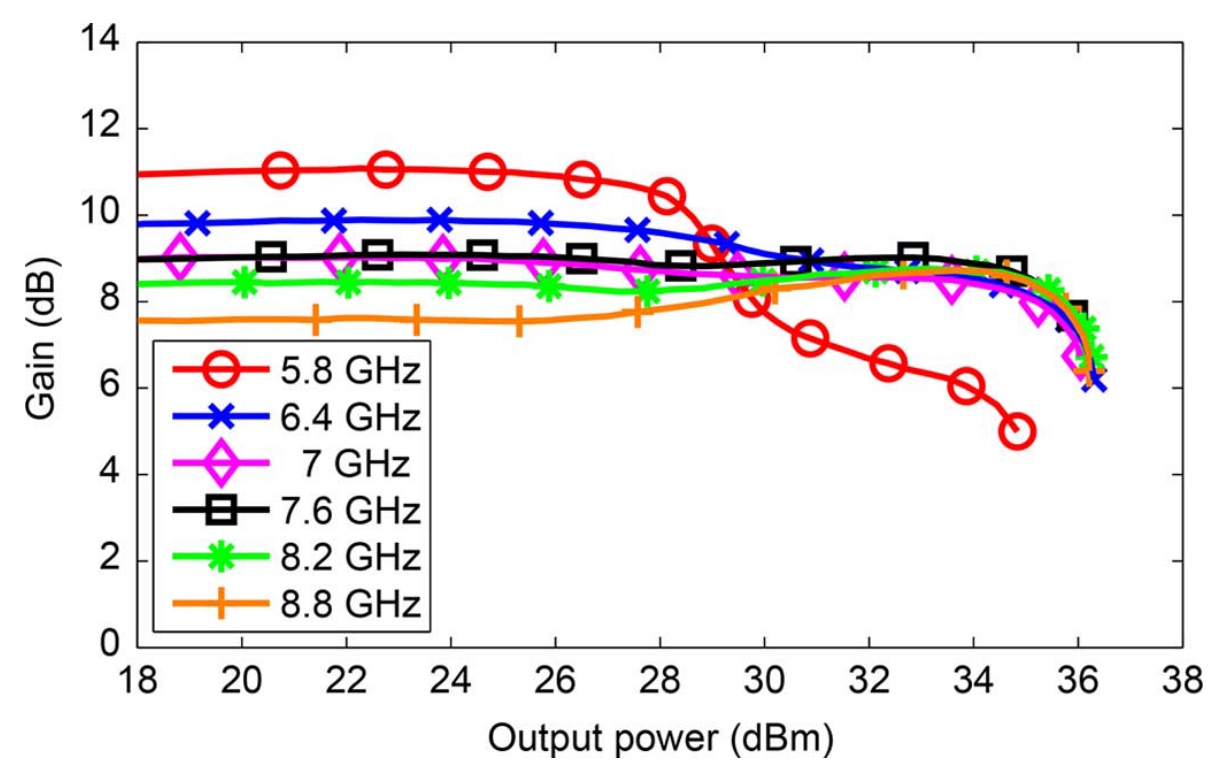

Figure II.15: Measured gain versus output power [45]

In [46] using tunable capacitors in the input and output matching networks, a $2.45 \mathrm{GHz}$ GaN HEMT operating class and frequency reconfigurable power amplifier is reported. Figure II.16 and Figure II.17 show, respectively, the concept of impedance matching conditions and a topology of the photograph of the proposed reconfigurable PA. The proposed PA can be reconfigured to operate in class A or class B to achieve, respectively, an extreme high linearity and high efficiency where high linearity is not strictly required. As a result, the proposed reconfigurable PA design can be reconfigured for multiple bands and for switching between operating classes. This reconfigurability is clearly seen in Figure II.18, Figure II.19 and Figure II.20 where gain, output power and PAE versus the swept input power, respectively, are plotted. 


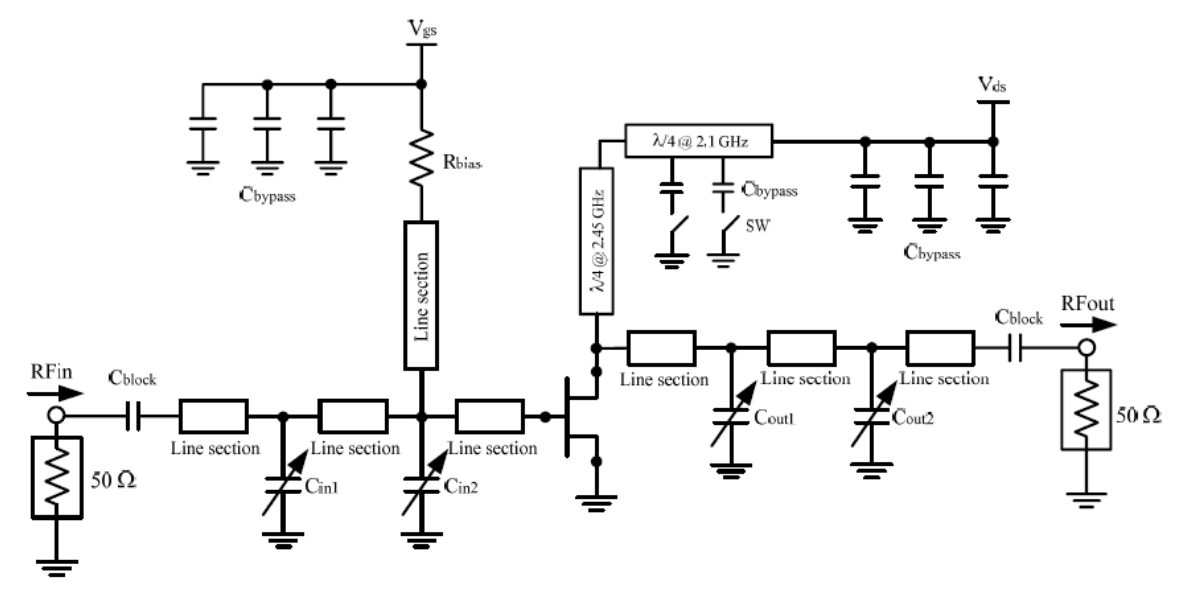

Figure II.16: The concept of impedance matching conditions of the band and class reconfigurable power amplifier [46]

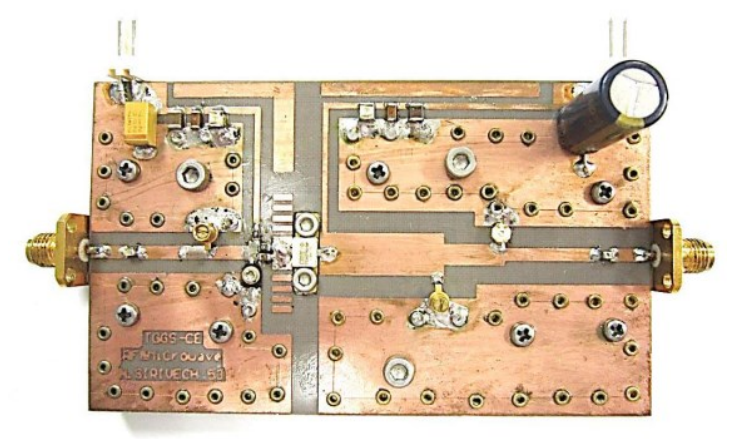

Figure II.17: Photograph of the fabricated class-reconfigurable power amplifier [46]

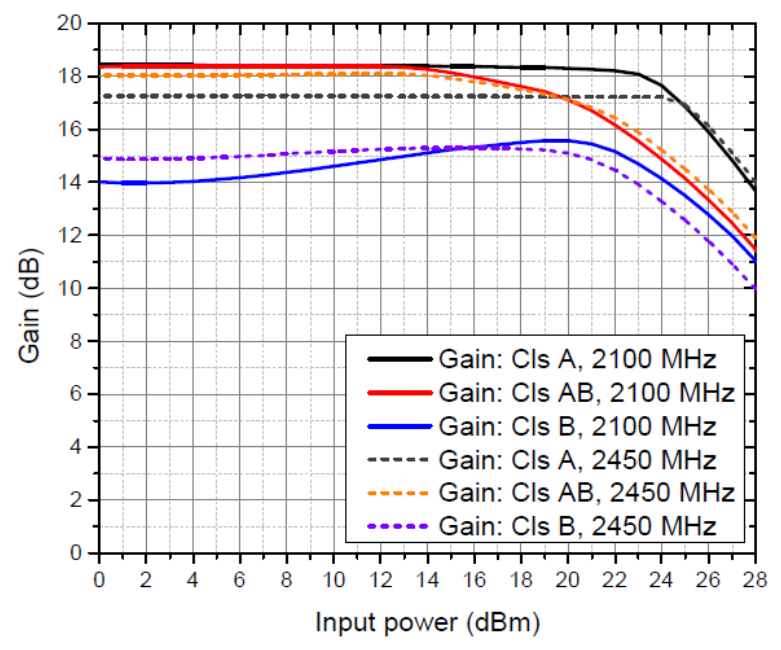

Figure II.18: Simulated gain verses input power at different matching states [46] 


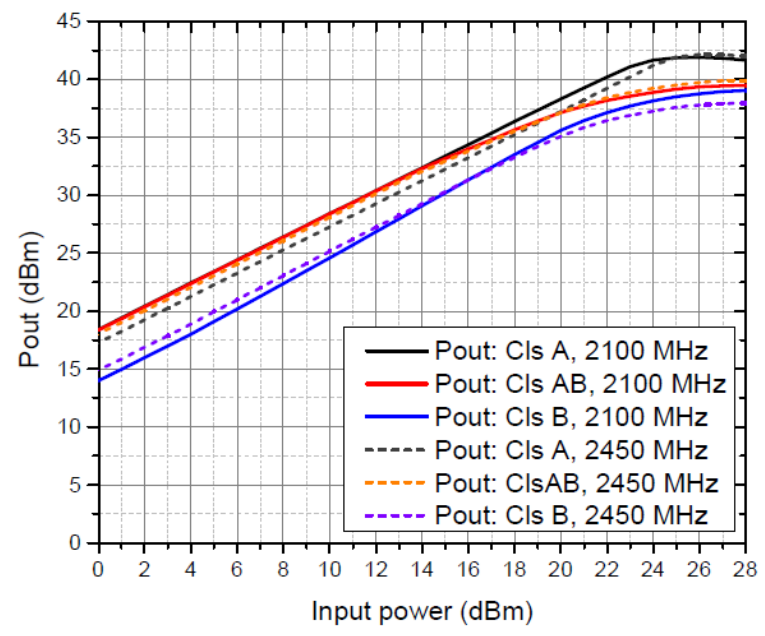

Figure II.19: Simulated output power verses input power at different matching states [46]

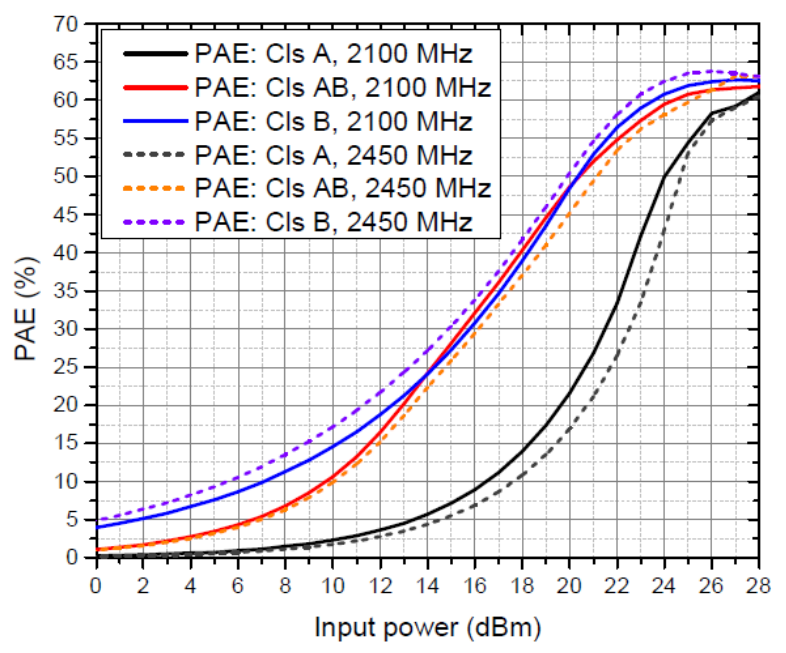

Figure II.20: Simulated power added efficiency verses input power at different matching states [46]

In [47], for the first time a novel technique on how to combine the advantages of the high power handling capabilities of GaN devices and microfluidic channels integrated in a multilayer organic substrate by designing a reconfigurable GaN-based PA at $2.4 \mathrm{GHz}$ and $5.8 \mathrm{GHz}$ is designed. An illustration of the packaged PA is shown in Figure II.21. The new technique regarding the design of tunable source and load matching networks frequency response is based on using different 
fluids (acetone for $2.4 \mathrm{GHz}$ and air for $5.8 \mathrm{GHz}$ ) injected inside their respective micromachined channels. As reported, the fabricated PA provides an output power of $35 \mathrm{dBm}$ with a PAE of $36.41 \%$ at $2.3 \mathrm{GHz}$ for an input power of $21 \mathrm{dBm}$, while at 5.7 $\mathrm{GHz}$ an output power of $36.88 \mathrm{dBm}$ is achieved with a PAE of $44.03 \%$ for an input power of $25 \mathrm{dBm}$. These measured results are plotted in Figure II.22 and Figure II.23 for, respectively, acetone and air configuration.
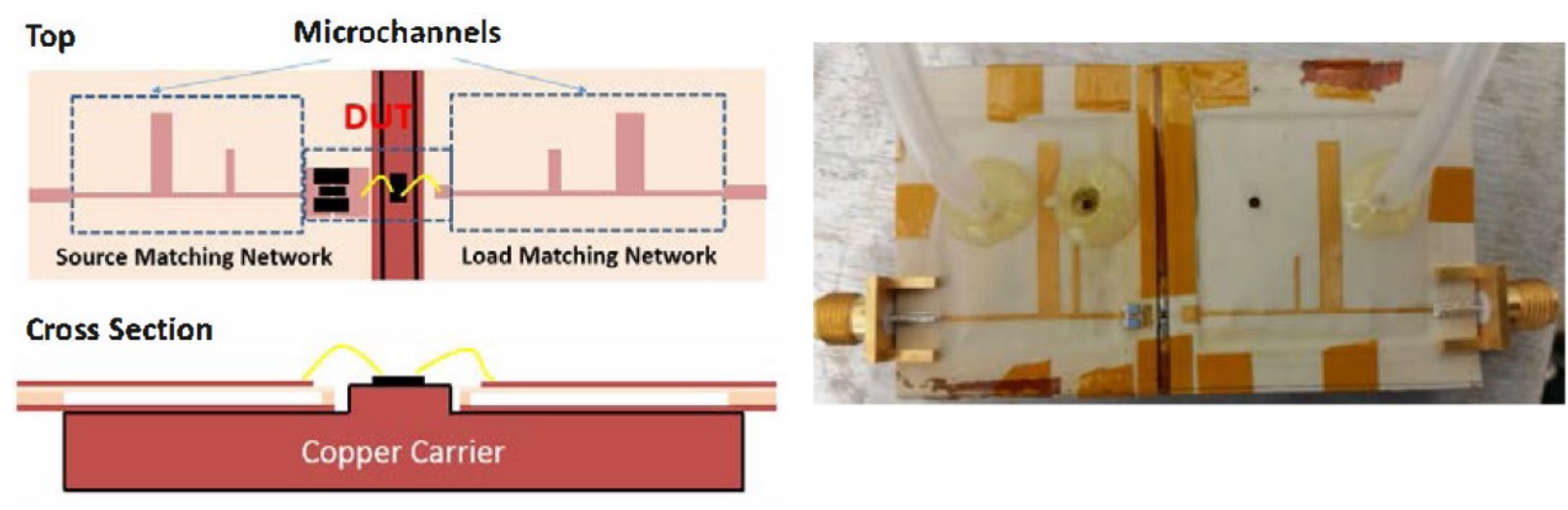

Figure II.21: (Left) Top and cross sectional view and (Right) Picture of the packaged $P A[47]$

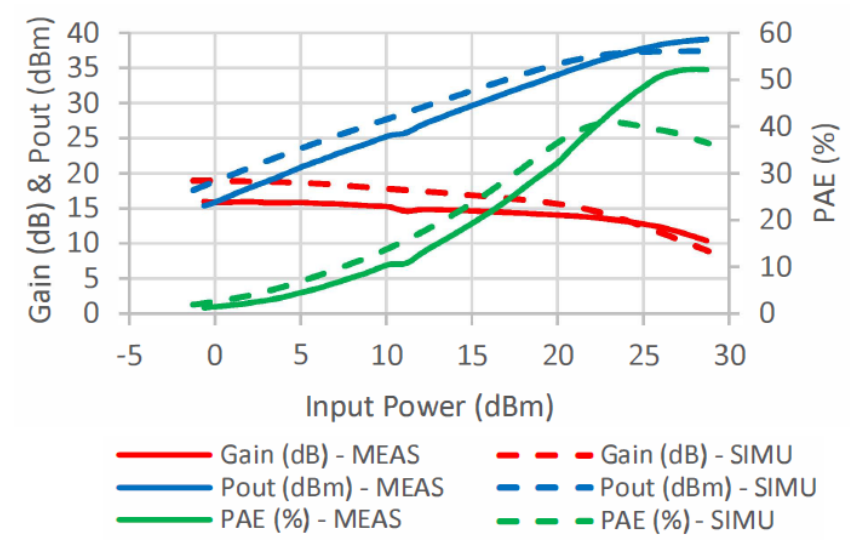

Figure II.22: Acetone configuration: measurements at $2.3 \mathrm{GHz}$, simulations at 2.4 $\mathrm{GHz}[47]$ 


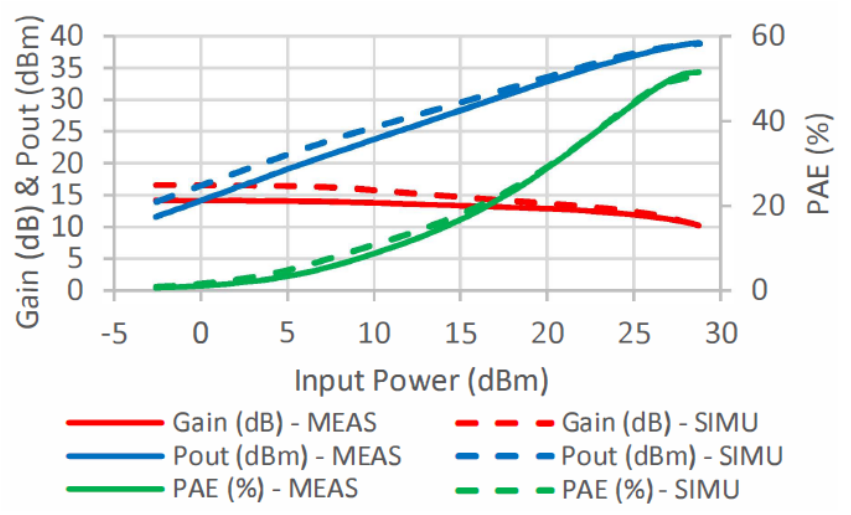

Figure II.23: Air configuration: measurements at $5.7 \mathrm{GHz}$, simulations at $5.8 \mathrm{GHz}$ [47]

More studies revealed that due to the drawbacks of GaAs MMIC HPA as low efficiency and limited output power level have made it not to be a competent candidate of power chips against the high efficiency of GaN MMICs technology [39]. In this same reference, a GaN-based high power amplifier with output power of $85 \mathrm{~W}$ and operating at S-band is reported. In 2014, an investigation [38] revealed that the next generation of GaN MMICs will cover more functionalities on the same chip. Multifunctional MMICs will allow very compact Tx/Rx module designs, and will reduce the number of active devices [38].

In this context, this thesis research is focused to demonstrate the use of GaN on highly efficient reconfigurable designs for broadband RF/microwave applications operating below $10 \mathrm{GHz}$. GaN components such as varactors and switches used for tunable purpose are firstly fabricated, characterized and then modeled. The proposed models are used to achieve the reconfigurability of $\mathrm{RF} /$ microwave circuits. Tunable phase shifter, reconfigurable $3^{-} \mathrm{dB} 90^{\circ}$ hybrid coupler, tunable frequency oscillator, beam switching antenna array and matching reconfigurable patch antenna are developed along with the development of GaN modeling varactors and switches, and are presented as applications results of this thesis research. A current literature review of these reconfigurable cited circuits will be presented along with the realization of these designs later in chapter IV. 


\section{Summary}

In this chapter the properties of GaN technology have been reviewed, discussed and compared with the commonly used semiconductor technologies. It has been clearly shown that GaN technology is a great promising semiconductor candidate for applications that high power and high frequency are required as well as high temperature. Its disadvantages and difficulties are also studied presented and discussed. Additionally the features and limitations of the used AlGaN/GaN process throughout this thesis provided by NRC were summarized and presented. Furthermore, an overview of the ability to use GaN technology for tunable purposes such as power amplifier discussed. We also reviewed some previous work done on the development of reconfigurable power amplifier circuits using GaN technology that are used in $\mathrm{Tx} / \mathrm{Rx}$ modules. 


\section{Chapter III}

\section{GaN Varactor and Switch Devices} Modeling

\section{Introduction}

A general purpose reconfigurable integrated $\mathrm{RF} /$ microwave circuit could be realized using a promising semiconductor technology for the active device, and an interconnection technique such as wire-bonding technique to compact all the circuits. Recent technology trends favor greater integration of electronic devices and radiating elements, greater functionality and reconfigurability (on a single chip or via multichips), as well as high operating frequency/power with excellent efficiency and linearity. Such are the needs for next generation of $\mathrm{Tx} / \mathrm{Rx}$ modules. 
For the proposed thesis research, GaN-based varactor and switch components will be pursued; the main features of this type of semiconductor technology being its high power with high efficiency. Compact reconfigurable circuits, such as tunable phase shifter, reconfigurable $3-\mathrm{dB} 90^{\circ}$ hybrid coupler, tunable frequency oscillator, beam switching antenna array and matching reconfigurable patch antenna based on these developed varactors and switches will be studied. The first part of the work is devoted to active GaN device development and characterization. The second part addresses the integration and design aspects of the reconfigurable proposed circuits.

GaN technology to date has seen very little application to signal control devices such as varactors and switches. These two elements are key points to realize phase shifters (both continuous and discrete state), frequency reconfigurable circuits, and signal path selection circuits for beam switching or Tx/Rx selection.

Our investigation considers modeling in small- and large-signal of both GaN varactor devices and GaN switch devices, as outlined in the "Work Plan" chart below (see Figure III.1). The design of these GaN components was realized using the Canadian National Research Council (NRC) GaN HEMTs monolithic microwave integrated circuit (MMIC) processes. Three NRC GaN HEMTs processes, such as, GaN150v0 with a gate length of $0.15 \mathrm{um}, \mathrm{GaN} 500 \mathrm{v} 1$ with gate length of $0.5 \mathrm{um}$ and GaN500v2 which is the developed version of GaN500v1, have been used to manufacture the varactor and the switch devices.

An accurate large signal model requires extensive laboratory equipment to extract all the effects related to high power GaN devices, such as thermal and trapping effects typically present in GaN devices. Carleton University is not as well equipped with these laboratory facilities as are the NRC or IETR. Due to our incapability to have large signal measurement facilities, only small signal measurement will be presented and discussed. However, for varactors fabricated 
using GaN500v2, we finally succeed to do large signal measurements. These measurements have been done at XLIM at University of Limoges, France.

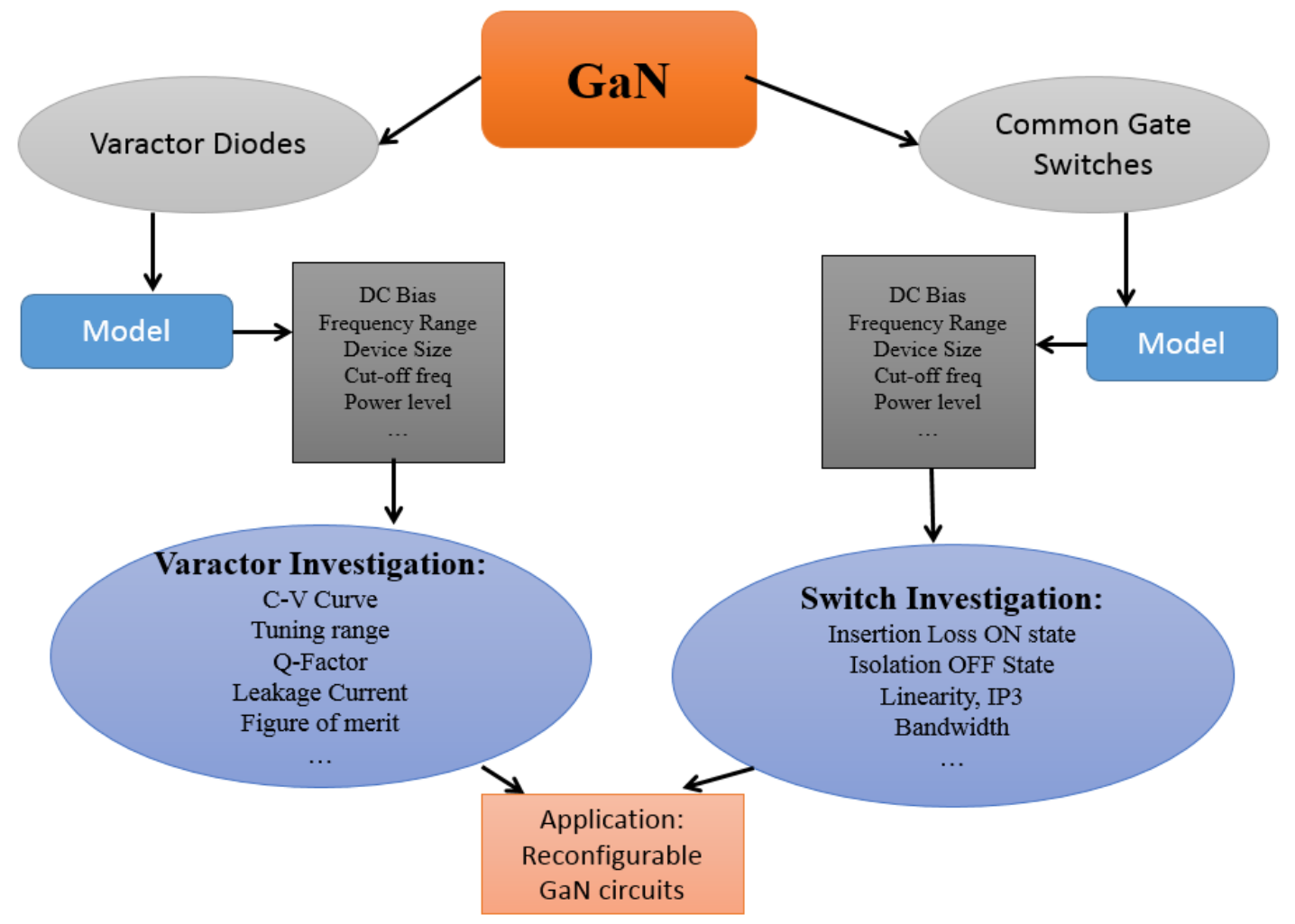

Figure III.1: Work Plan

First step, we start investigating varactor devices. For this, two NRC GaN HEMTs processes, GaN500 and GaN150, have been used to manufacture the devices. At this time the current version (v1) of GaN500 process was used; while for GaN150, the process was still under-development and its initial version (v0) was used which was non-commercialized one. Many size different devices have been manufactured and characterized in only small signal measurements, and then a small signal model has been implemented. This model describes clearly the behavior of GaN based varactor. Following the development of NRC GaN HEMTs process, another fabrication run has been launched to manufacture other varactor devices with different sizes and by using, in this time, only the newly developed version of GaN500 process, version 2 . This time, small signal as well as large signal measurements have 
been done on these novel realized varactors, respectively, to validate the proposed small signal model developed from GaN500v1 process' devices and to verify their capabilities in terms of different power levels. Secondly, single GaN switches with different sizes have been fabricated and measured in only small signal. A model is presented and discussed. These varactor and switch models will be used to simulate and design reconfigurable $\mathrm{RF} /$ microwaves designs shown in the chapter IV.

\section{GaN-based Varactors Diodes Modeling}

\section{Review of GaN HEMT for Power Varactors Diodes Applications}

GaN HEMTs semiconductor devices are no longer limited to RF power amplifier applications, but having been extended to other applications such as LNAs [48], VCOs [49], mixers [51], and phase shifters [50]-[52]. Furthermore, reconfigurable $\mathrm{RF}$ circuits, with high power/frequency, and excellent linearity, are in great demand. Varactors are key components in the design of these reconfigurable circuits [53] for microwave and millimeter wave applications. The varactor diodes can be fabricated from either p-n or Schottky junctions and both cases are under research today.

The superior characteristics (i.e high-temperature operation due to large energy band gaps, high breakdown field, high saturation velocities of electrons, etc) make GaN heterostructures promising candidates for realizing high power/tuning voltage varactors diodes. Up to now, the progress on developing GaN varactors have been limited and only a few works have been already reported. Based on GaN HEMT technology, some varactors have previously been reported, mostly employing a MetalSemiconductor-Metal (MSM) junction [53]-[54]-[55]-[56] or Schottky barrier contact [57]-[58]-[59].

Later 2002, one of the first investigated GaN varactor work was reported in [53]. This work investigates the properties of MSM diodes that are based on an 
AlCaN/GaN HEMT layer structure (MSM-2DEG), which shown that devices made in this material system are realizable with tuning capacitance range. As can be seen from Figure III.2, the capacitance is constant between 2.7 and $-2.7 \mathrm{~V}$ and within a very small voltage range of about $0.3 \mathrm{~V}$ the capacitance starts getting changed. The reported measured $\mathrm{C}_{\mathrm{MAX}} / \mathrm{C}_{\mathrm{MIN}}$ ratios vary from 5 to 100 and were dependent on electrode geometry. This investigation shown clearly that these ratio values were exceeding those ratios for varactor diodes in GaAs. However, a high series resistance varies from $40 \Omega$ to $70 \Omega$ was extracted and these values were dependent on the electrode spacing from $0.5 \mathrm{um}$ to $2 \mathrm{um}$. This high series resistance of the device, which was announced as a challenge for varactors diodes, can affect directly the figure of merit, quality factor ( $Q$-factor), of the device. In addition, this high value of series resistors is due to the unavoidable ohmic contact resistance. Researchers have begun to investigate the possibilities in order to reduce this specific contact resistance.

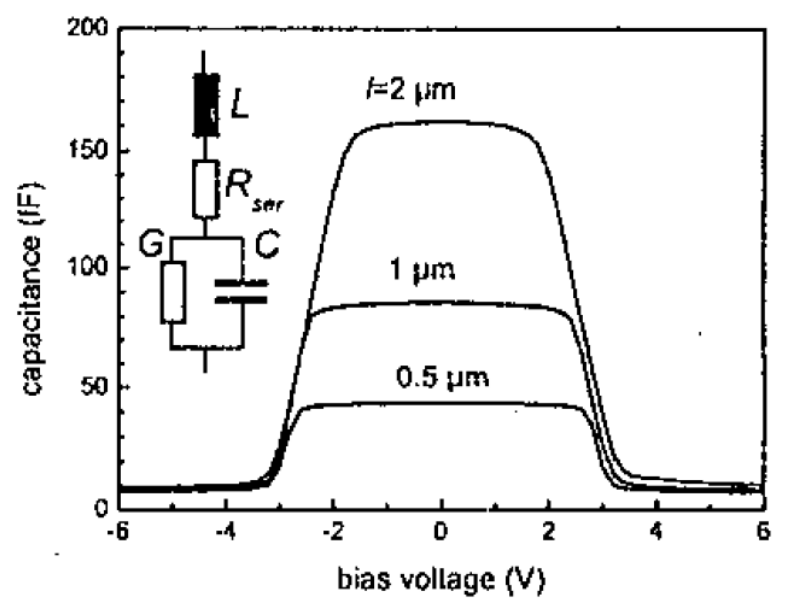

Figure III.2: $C$-V characteristics of devices with different electrode length (spacing = $1 \mathrm{um}$, width $=50 \mathrm{um}$ ) [53]

In [54], a work focused on characterizing the Q-factor of MSM AlGaN/GaN varactors that operate in $\mathrm{RF}$ range was reported. The ohmic contact is eliminated and replaced by a Schottky contact, resulting in reduced overall series resistance and higher peak $\mathrm{Q}$-factor. A standard AlGaN/GaN HEMT process was fully compatible for manufacturing this MSM varactors. The $\mathrm{C}-\mathrm{V}$ measurements (see Figure III.3) 
revealed that the device was capable to valid the rapid change on capacitances values over a small range of bias voltage. At $1.1 \mathrm{GHz}$, the capacitance tuning range was varying from 1.62 to $0.12 \mathrm{pF}$ corresponding to a $\mathrm{C}_{\mathrm{MAX}} / \mathrm{C}_{\mathrm{MIN}}$ ratio of 13.5 . Moreover, a high series resistors values were still achieved. The dependence of the $\mathrm{Q}$-factors on the bias voltage at $1.1 \mathrm{GHz}$ is illustrated in Figure III.4. From both Figure III.3 and Figure III.4, it can be seen that the value of $\mathrm{Q}$-factor decreased as well as the series resistor value increases. A physical equivalent circuit model was reported to explain the behavior of the voltage controlled capacitance and the corresponding $\mathrm{Q}$-factor. It was revealed that the fundamental limit on the $\mathrm{Q}$-factor in the intermediate capacitance range is the rapidly increasing channel resistance when the channel is depleted. Although, it was reported that further improvement of the $\mathrm{Q}$-factor has to come from modification of the conventional single-channel HEMT using double- or multiple-channel structure in which additional low impedance current paths are available.

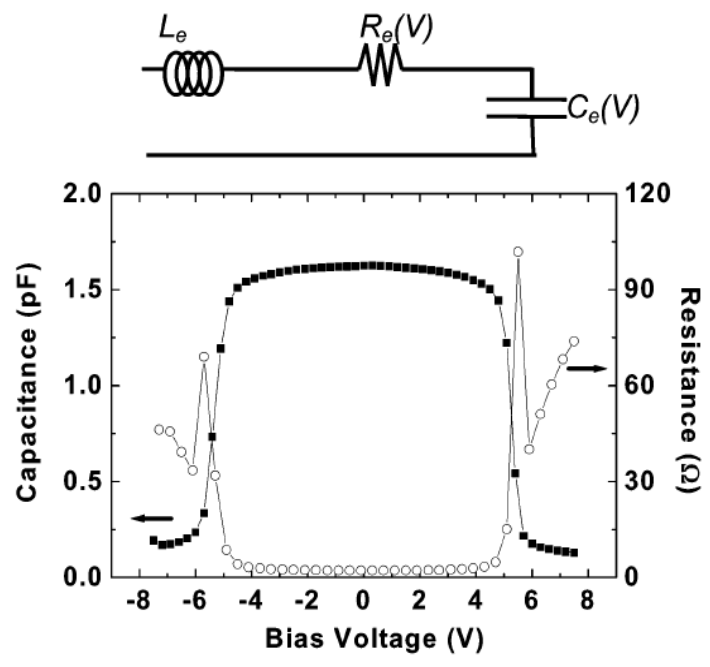

Figure III.3: Extracted capacitance (black line) and resistance (white line) of the MSM varactor over the entire bias range at $1.1 \mathrm{GHz}$. The inserted schematic shows the first-order equivalent circuit of the MSM varactor [54] 


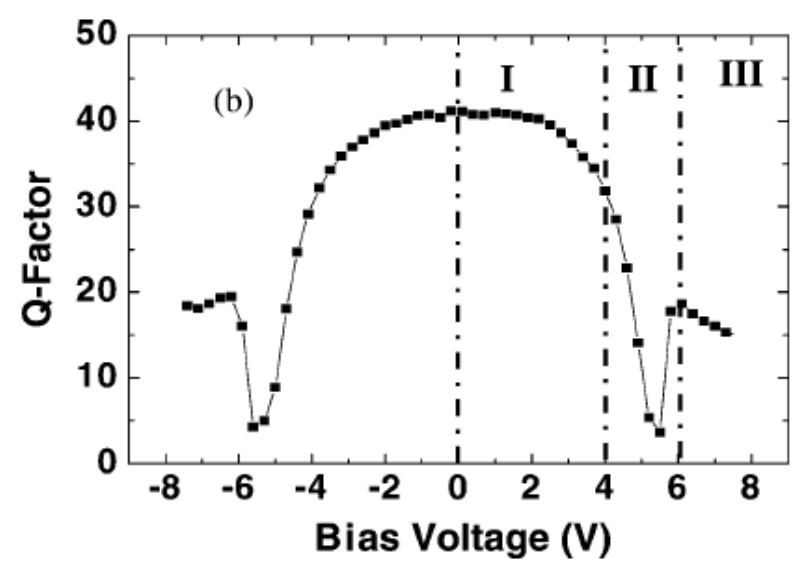

Figure III.4: Q-factor versus tuning bias at $1.1 \mathrm{GHz}[54]$

Another investigation MSM GaN varactor was reported in 2012 in [55]. In this work, a MSM varactor diodes on top of an AlGaN/GaN-based epitaxial structure is proposed to the fast surge protection application. This works was focused on developing a surge to protect electronic devices against the natural or manmade surge threats, including lightning, electromagnetic pulse (EMP), electrostatic discharge (ESD) etc. Figure III.5 shows the schematic diagram of an AlGaN/GaN-based varactor used in this study while the $\mathrm{C}-\mathrm{V}$ curves of the surface untreated and the sulfur-treated AlGaN/GaN-based MSM-2DEG varactors used in this study are presented in Figure III.6. This investigation showed that the varactor exhibits great potential to improve the surge protection for GaN-based HEMTs. Therefore, the fabricated process significantly affects the capacitance swing of MSM-2DEG varactors. Due to the variable impedance characteristics of the varactor, it is either within the high impedance region for blocking the surge energy or within the low impedance region for passing normally the signal. 


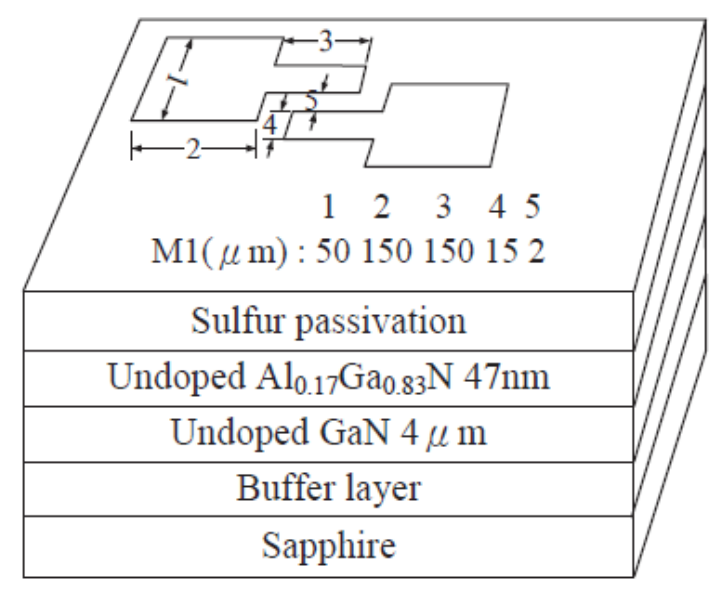

Figure III.5: A Schematic diagram of the AlGaN/GaN-based varactor [55]

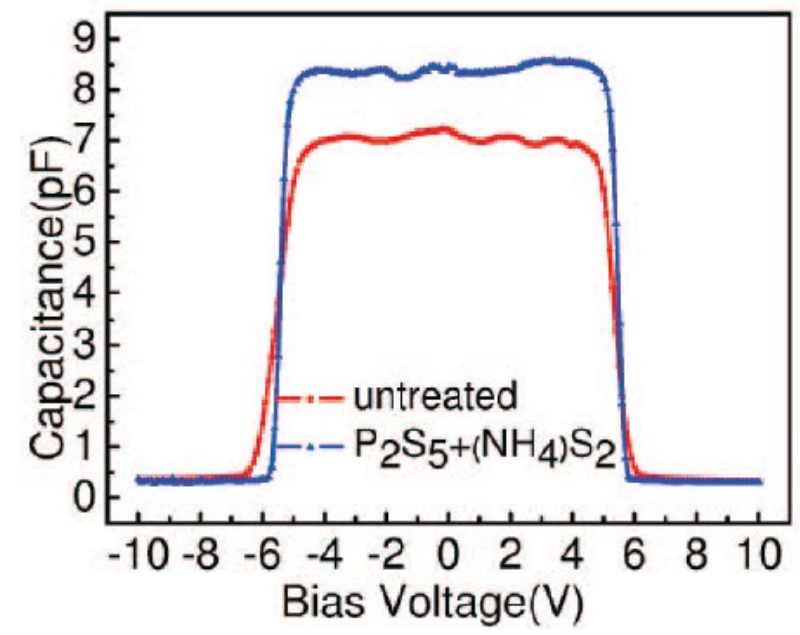

Figure III.6: Measured C-V curves of the untreated and sulfur-treated AlGaN/GaNbased MSM-2DEG varactors [55]

In 2015, by the same group researchers of [55], a MSM varactor study has been reported in [56]. Here, the performance of the AlGaN/GaN MSM-2DEG varactor with different dielectric films deposited by the E-beam deposition is investigated. In addition, different dielectric films are studied to verify the effect of the dielectric material on the capacitance ratio and the bias voltage of varactors. For this, three dielectric films have been used, such as, $\mathrm{SiO} 2, \mathrm{Gd} 2 \mathrm{O} 3$, and $\mathrm{Si} 3 \mathrm{~N} 4$ to conduct this study. Figure III.7 illustrates the schematic diagram of the AlGaN/GaN MSM-2DEG varactor used for this study. From Figure III.8, it was observed that the capacitance 
tuning ranges are almost identical irrespective of whether with dielectric film or not. However, the capacitance ratio $\left(\mathrm{C}_{\mathrm{MAX}} / \mathrm{C}_{\mathrm{MIN}}\right)$ for each varactor is affected by the type of the dielectric film used. It was proved that the varactors with the $\mathrm{SiO} 2$ and Gd2O3 dielectric films offer the benefit of a lower leakage current. It was also observed that the $\mathrm{C}_{\mathrm{MAX}}$, the $\mathrm{C}_{\mathrm{MIN}}$ and the capacitance ratios show the same tendency of decreasing for varactors with dielectric films and are proportional to the increasing of the electrode areas.

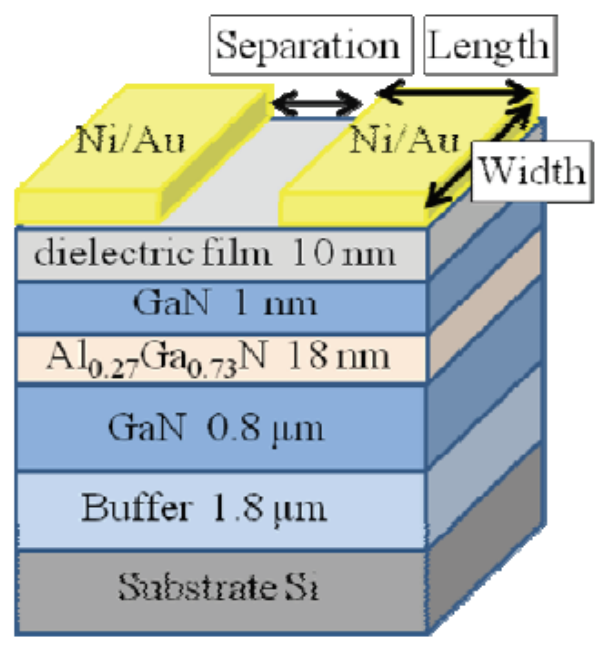

Figure III.7: Schematic diagram of the AIGaN/GaN MSM-2DEG varactor of the samples prepared in this study [56] 
(a)

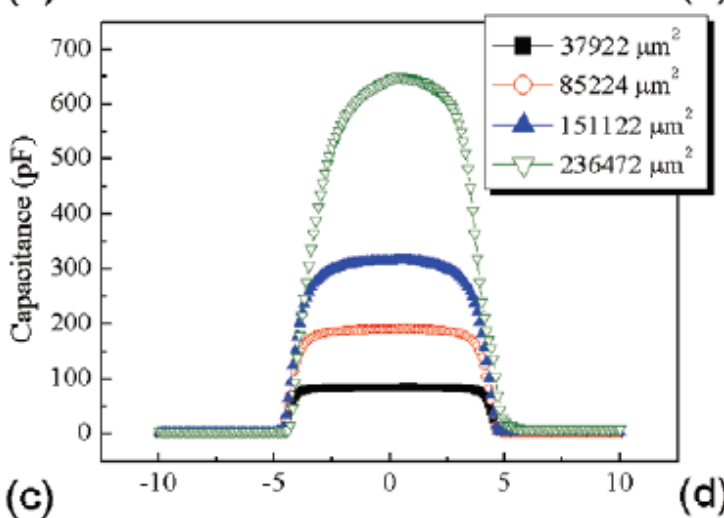

(b)

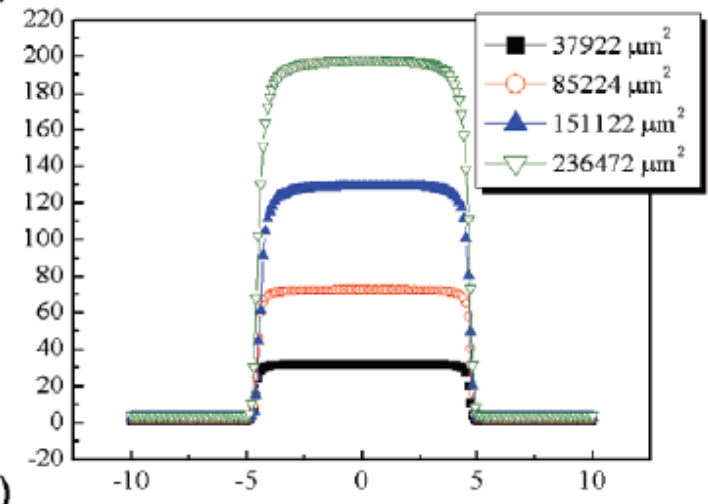

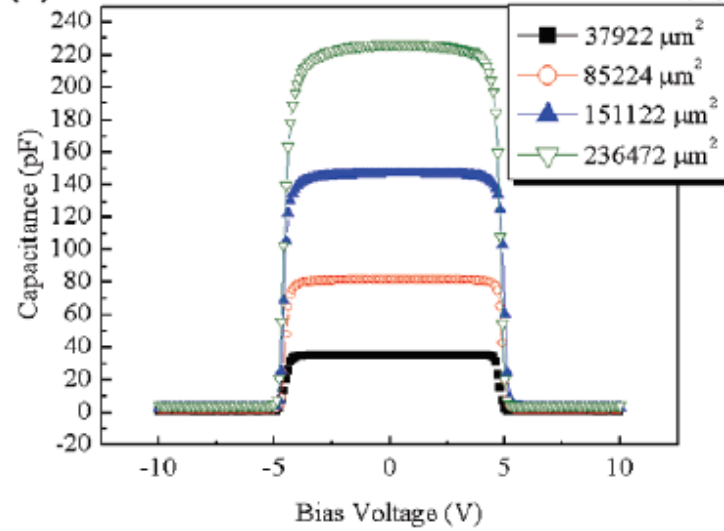

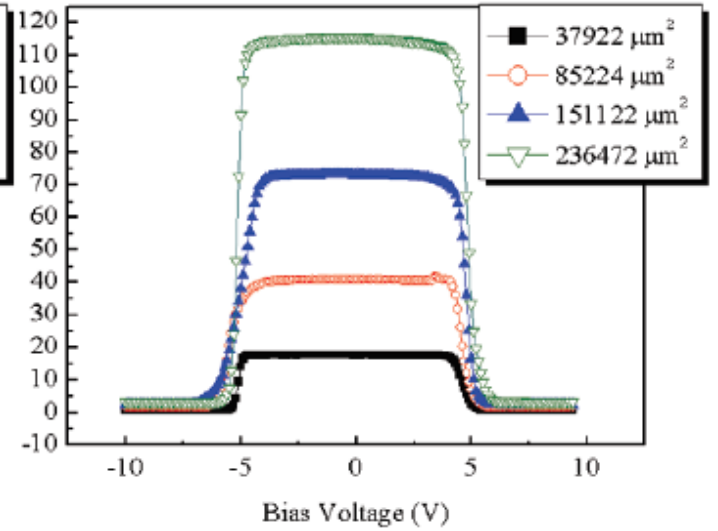

Figure III.8: Capacitance-Voltage ( $C$-V) characteristics of varactors with different electrode areas (a) without any dielectric film, (b) with SiO2 film, (c) with Gd2O3 film, and (d) with Si3N4 film. Measurement frequency $=1 \mathrm{MHz}$, electrode area $=$ 37922, 85224, 151122, and $236472 \mathrm{\mu m}^{2}$, respectively [56]

In [57], a novel GaN-based high frequency varactor diode was reported. This main goal of this work is to investigate the high frequency performances expected from such GaN-based Schottky barrier varactor diode. The structure used in this work consists of a $2 \mu \mathrm{m}$ bottom ohmic $\left(\mathrm{n}^{+}\right)$contact layer and a $300 \mathrm{~nm}$ lightly doped ( $n^{-}$) GaN layer which is grown on top of the ohmic layer. The ohmic contacts (Ti/Al/Ti/Au) were deposited once the mesa mask was isolated by Ar etching and then Pt was deposited which forms the Schottky contact metal. The schematic of the GaN Schottky varactor structure is shown in Figure III.9. It was shown that epilayer designs can help in controlling such characteristics. As shown in Figure III.10, the 
maximum capacitance at $0 \mathrm{~V}$ bias is $40.476 \mathrm{fF}$ and the minimum capacitance is about $26.4 \mathrm{fF}$ when a bias of less than $-7 \mathrm{~V}$ is applied. This reported varactor had a breakdown voltage of $20 \mathrm{~V}$ and a cut-off frequency of $360 \mathrm{GHz}$.

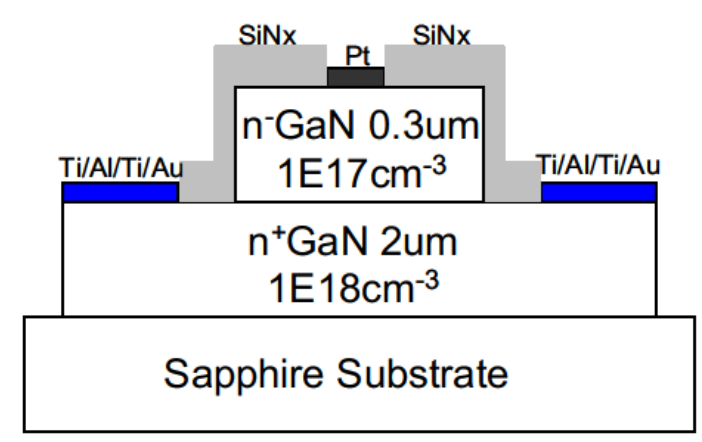

Figure III.9: Schematic Cross-sectional view of the GaN Schottky Varactor [57]

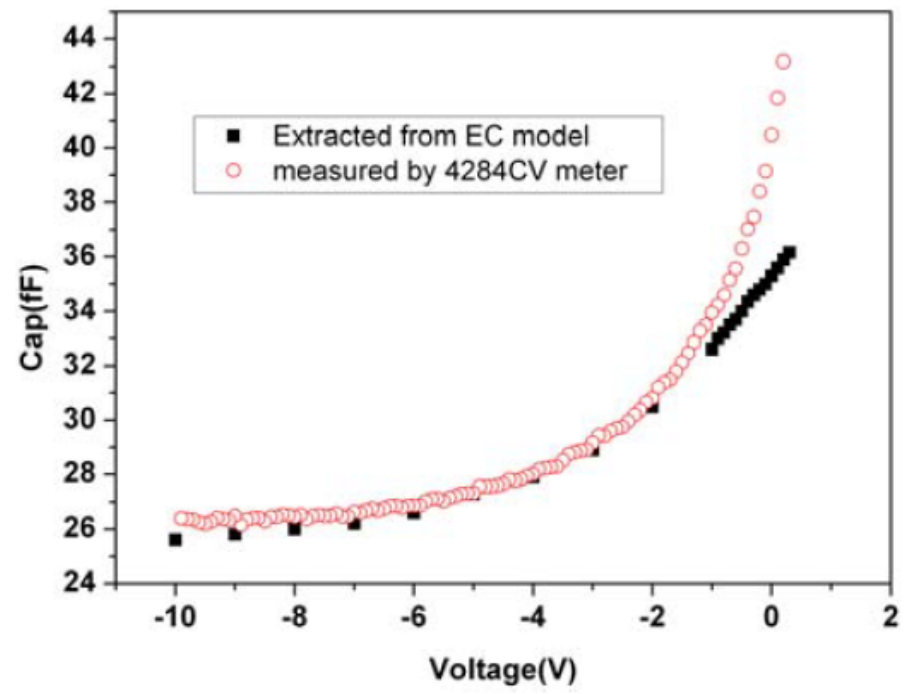

Figure III.10: C-V characteristics of the GaN Schottky Varactor [57]

Based on Schottky barrier, another work was reported in [58]. In this work, a design of InGaN/GaN schottky diodes with enhanced voltage handling capability with vertical geometry was discussed. It is, therefore, illustrated that a higher voltage handling capability may be obtained with a suitable InGaN surface layer design. Figure III.11 schematically shows the structure of a GaN varactor. Furthermore, the depletion width $\mathrm{W}$ varies in the $\mathrm{n}^{-}$region under several external bias voltage, this resulting to a change in equivalent $\mathrm{C}$ as well as in its associated series resistors $\mathrm{R}$. 
The reported work shows that varactor diode made in GaN material are capable of bias voltages over $120 \mathrm{~V}$ at a doping level of $1 \times 10^{17} \mathrm{~cm}^{-3}$ over a 1 - $\mu$ m-thick depletion region. Furthermore, at $1 \mathrm{GHz}$, a significantly high $\mathrm{Q}$ value superior of 35 was reported which corresponds to series resistance of this device $(\sim 8 \Omega)$ which was primarily from the underlying $\mathrm{n}^{+} \mathrm{GaN}$ and contact resistance. Figure III.12 shows the $\mathrm{C}-\mathrm{V}$ curves over the range of $0-100 \mathrm{~V}$ for the InGaN-containing samples and for a control sample.

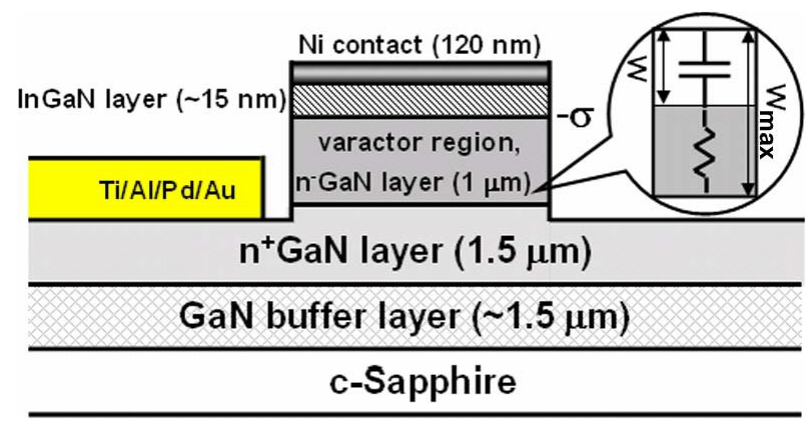

Figure III.11: Schematic diagram of a GaN-based varactor and its layer structure [58]

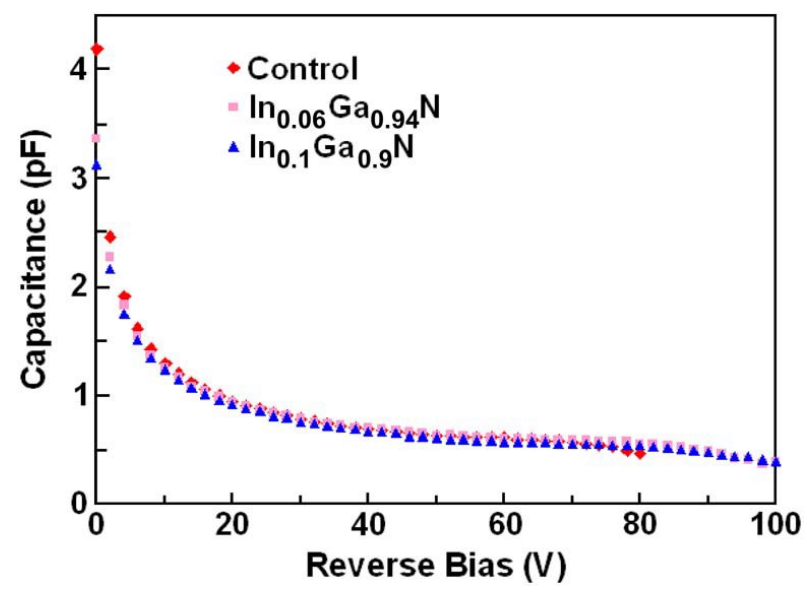

Figure III.12: $C$-V curves measured for the samples with area of $5 \times 10-5 \mathrm{~cm} 2$ at $f=$ $1 \mathrm{MHz}$ [58] 
Two years later, on 2012, the same group researchers of [58] reports a new work [59] where the critical design considerations for achieving GaN-based high voltage, high $\mathrm{Q}$ factor and high linearity microwave varactors were summarized and were discussed. In addition, detailed performances for a real fabricated GaN-based anti-parallel varactor diode structure that simultaneously achieving high $\mathrm{Q}$, highbreakdown voltage and high linearity were presented. This investigation was based on using the same GaN structure presented in Figure III.11. It was highlighted that heavily doped semiconductor material is required to minimize the series resistor and achieve a high $\mathrm{Q}$-factor. However, in order to achieve high breakdown voltage, the semiconductor needs to be lightly doped to keep the electric field in the semiconductor below its critical electric field. Furthermore, the two anti-parallel diodes structure was used to investigate the varactor linearity. It was reported that by using this structure, the RF signal induced capacitance variation is minimized. The linearity of the varactor diode is thus significantly improved. Figure III.13 shows the simulated $\mathrm{C}-\mathrm{V}$ curves for single and two anti-parallel structures, while in Figure III.14, Q-factor versus the reverse external bias is depicted.

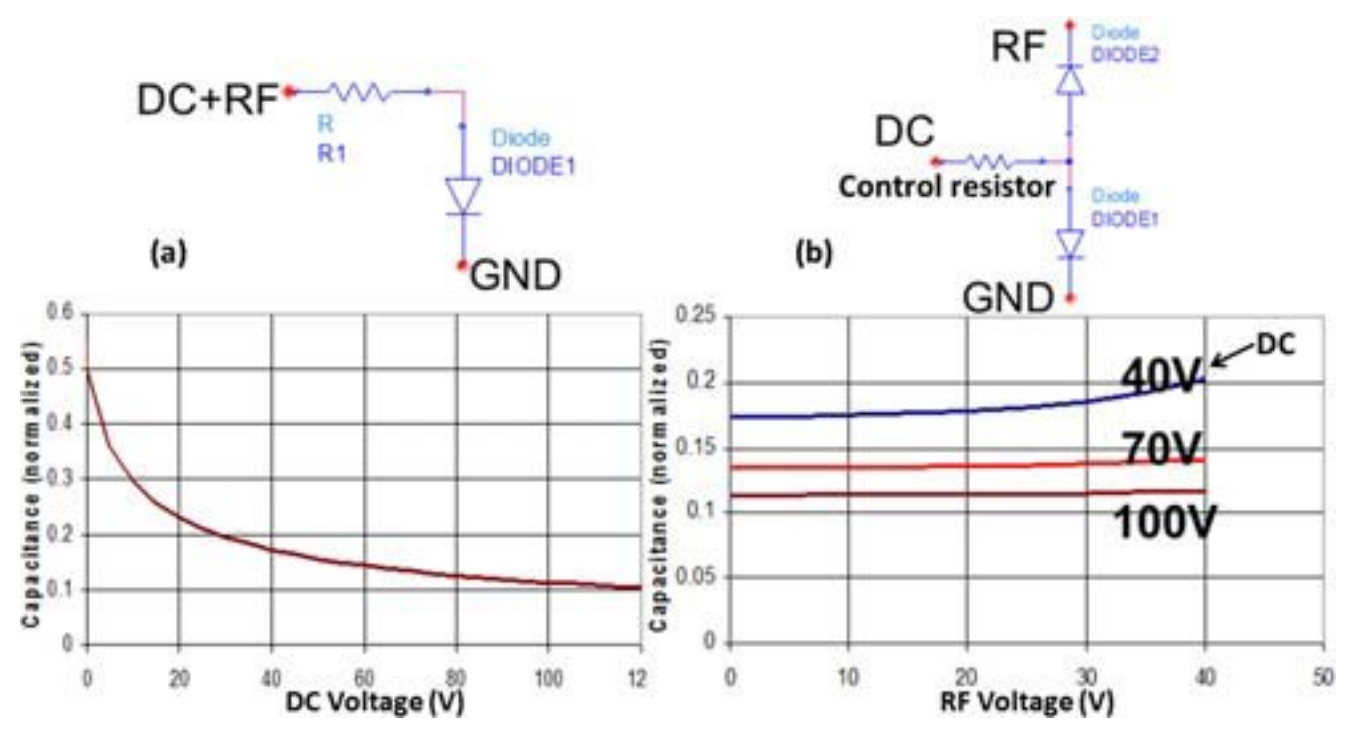

Figure III.13: Capacitance-voltage curves for (a) single diode structure and (b) two anti-parallel diode structure [59] 


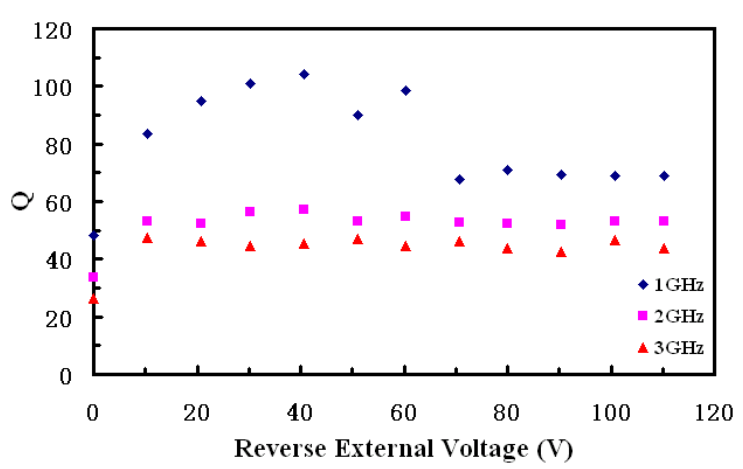

Figure III.14: $Q$ factor versus reverse external bias for an anti-parallel varactor at $f$ $=1 \mathrm{GHz}, 2 \mathrm{GHz}$ and $3 \mathrm{GHz}$. Each diode has an area of $3.8 \times 10-5 \mathrm{~cm}^{2}$ [59]

\section{Realized GaN HEMT-based Varactor Diodes}

These previous reported works present good performance in term of high breakdown voltage, high $\mathrm{Q}$-factor, and high linearity; however, the limited capacitance tuning range is still a challenge. Furthermore, to achieve good performance, most of these reported works require some changes in epitaxial and mask layer of the GaN HEMT process which results in a complexity of fabrication and cost increasing. These challenges have limited the progress of developing varactor diodes. In order to overcome the two above-mentioned drawbacks, here, for this thesis work, the varactor diode is realized by connecting together the drain and source terminations thus resulting in a single Schottky junction which can be considered as planar schottky varactor diode (PSVD) [68].

The bias potential $V_{G}$ is then applied across the drain/source and gate terminations. This kind of varactor diodes do not require any additional selective changing in the epitaxial layers of the HEMT process. As a result, a standard GaN HEMT process can be used which minimize their cost production compared to the other reported type of varactor diodes. However, GaN-based PSVDs have not been widely used because of the unavailability of accurate large and small signal models which become our motivations to develop them. We investigate the equivalent 
capacitance $\mathrm{C}_{\mathrm{Eq}}$ and the equivalent series resistance $\mathrm{R}_{\mathrm{Eq}}$ of the varactor, when the overall impedance is considered as a simple series RC circuit.

\section{GaN500v1 and GaN150v0 Varactor Devices}

Figure III.15 illustrates the layout design for one varactor device. To compare between the two processes (GaN500v1 and GaN150v0), two samples of each process with different fingers width are studied as detailed in Table III.1:

Table III.1: Presented GaN500v1 and GaN150vO Devices Information

\begin{tabular}{|c|c|c|c|c|}
\hline $\begin{array}{c}\text { Device } \\
\text { Terminology }\end{array}$ & $\begin{array}{c}\text { Number of } \\
\text { Fingers }\end{array}$ & $\begin{array}{c}\text { Fingers } \\
\text { Width }\end{array}$ & $\begin{array}{c}\text { Fingers } \\
\text { Length }\end{array}$ & $\begin{array}{c}\text { Process } \\
\text { Terminology }\end{array}$ \\
\hline W40L0.5 & $\mathbf{2}$ & $\mathbf{4 0} \boldsymbol{\mu m}$ & $\mathbf{0 . 5 0} \boldsymbol{\mu m}$ & GaN500v1 \\
\hline W100L0.5 & $\mathbf{2}$ & $\mathbf{1 0 0} \boldsymbol{\mu m}$ & $\mathbf{0 . 5 0} \boldsymbol{\mu m}$ & GaN500v1 \\
\hline W50L0.15 & $\mathbf{2}$ & $\mathbf{5 0} \boldsymbol{\mu m}$ & $\mathbf{0 . 1 5} \boldsymbol{\mu m}$ & GaN150 \\
\hline W100L0.15 & $\mathbf{2}$ & $\mathbf{1 0 0} \boldsymbol{\mu m}$ & $\mathbf{0 . 1 5} \boldsymbol{\mu m}$ & GaN150 \\
\hline
\end{tabular}

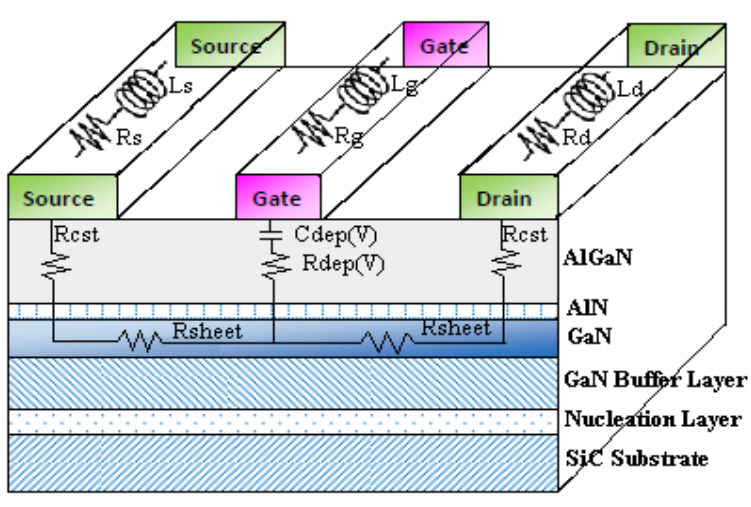

Figure III.15: Schematic cross-section of HEMT, showing contacts and epitaxial structures, and physical equivalent circuit origin at pinch-off $\left(V_{G}=-3.5 \mathrm{~V}\right)$

The W40L500v1 device is shown in Figure III.16 when it is positioned in Microwave probe test. 


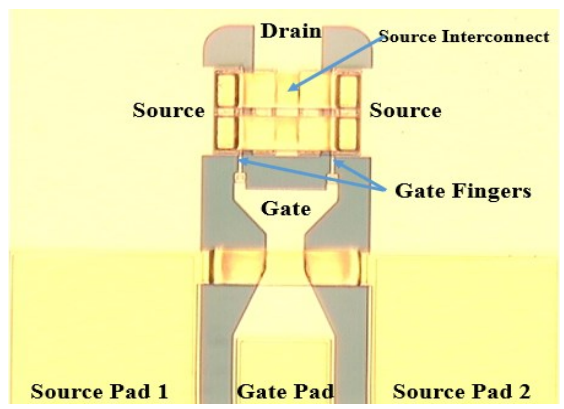

Figure III.16: W40L500v1 Device in Microwave Probe Test

\subsection{Small Signal Modeling}

The physical origins of the varactor device's equivalent model of both NRC GaN process are shown in the cross-sectional view in Figure III.15. $R_{g}, L_{g}, R_{d}, L_{d}, R_{s}$ and $L_{s}$ are, respectively, the metal resistance and the parasitic inductance of the gate, of the drain and of the source. $R_{\text {cst }}$ is the constant resistor and is determined by the AlGaN layer thickness. For GaN500v1 process, the thickness is about 20nm and $R_{\text {cst }}$ is $1 \Omega . \mathrm{mm}$. $\mathrm{R}_{\text {sheet }}$ refers to the channel resistance which is also constant and is determined by the two dimensional electron gas (2DEG), it is $300 \Omega / \square$, while $R_{\text {dep }}$ represents the resistance under the depletion region which is affected by the bias voltage, $\mathrm{V}_{\mathrm{G}}$, and changes slightly when the channel starts to deplete, this leads to a change in the series resistor of the equivalent circuit. $\mathrm{C}_{\mathrm{dep}}$ represents the depletion capacitance; it changes significantly with $\mathrm{V}_{\mathrm{G}}$. The physical equivalent circuit shown in Figure III.15 could be transformed to a simple equivalent RC circuit model as shown in Figure III.17.

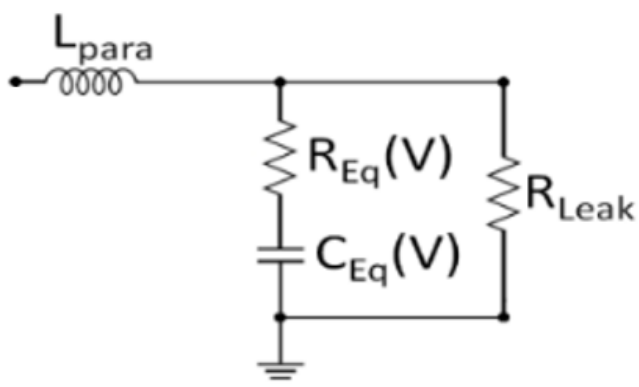

Figure III.17: Varactor equivalent circuit model 
$\mathrm{L}_{\mathrm{para}}, \mathrm{R}_{\mathrm{Eq}}, \mathrm{C}_{\mathrm{Eq}}$ and $\mathrm{R}_{\mathrm{Leak}}$, respectively, are the parasitic inductor, the equivalent series resistor, the equivalent series capacitance and the leakage resistor when the diode presents forward conduction current flow. The effect of $\mathrm{L}_{\text {para }}$ is negligible at low frequencies while the value of $R_{\text {Leak }}$ is very high (about $8 \mathrm{G} \Omega$ ). The on-wafer RF measurements were performed with an Agilent VNA Network Analyzer over a frequency range of $0.1-20 \mathrm{GHz}$ with a $\mathrm{RF}$ power of $-15 \mathrm{dBm}$. As can be seen in Figure III.18, Figure III.19, Figure III.20 and Figure III.21, a good match between the experimental results and the simulation model results is generally achieved. For GaN150v0, agreement is nearly perfect, while for GaN500v1 there are some issues with the larger device at high frequencies for $-6 \mathrm{~V}$ bias. Here the channel is fully depleted and some parasitic inductances are not captured in the equivalent model. For $\mathrm{V}_{\mathrm{G}}$ of $-3 \mathrm{~V},-4 \mathrm{~V}$ and $-6 \mathrm{~V}$, the parameter values of the equivalent model are presented in Table III.2.

Table III.2: Parameters values of the equivalent circuit

\begin{tabular}{|c|c|c|c|c|}
\hline Device & $\mathrm{V}_{\mathrm{G}}(\mathrm{V})$ & $\mathrm{L}_{\mathrm{para}}(\mathrm{pH})$ & $\mathrm{R}_{\mathrm{Eq}}(\Omega)$ & $\mathrm{C}_{\mathrm{Eq}}(\mathrm{fF})$ \\
\hline W40L0.5 & $-\mathbf{3}$ & $\mathbf{2 5}$ & $\mathbf{1 7}$ & $\mathbf{2 0 5}$ \\
\cline { 2 - 5 } & -4 & 25 & 50 & 190.7 \\
\cline { 2 - 5 } & -6 & 25 & $\mathbf{1 5 . 5}$ & $\mathbf{8 2 . 7}$ \\
\hline W100L0.5 & -3 & 30 & 6.9 & 533 \\
\cline { 2 - 5 } & -4 & 30 & 13.3 & 439 \\
\cline { 2 - 5 } & -6 & 30 & 6.16 & $\mathbf{2 2 0}$ \\
\hline W50L0.15 & -3 & 10 & 5 & 148 \\
\cline { 2 - 5 } & -4 & 10 & 9.7 & 98 \\
\cline { 2 - 5 } & -6 & 10 & 4.77 & 40 \\
\hline W100L0.15 & -3 & 20 & 4.9 & 286 \\
\cline { 2 - 5 } & -4 & 20 & $\mathbf{8 . 3}$ & 189 \\
\cline { 2 - 5 } & -6 & 20 & 5 & 81 \\
\hline
\end{tabular}




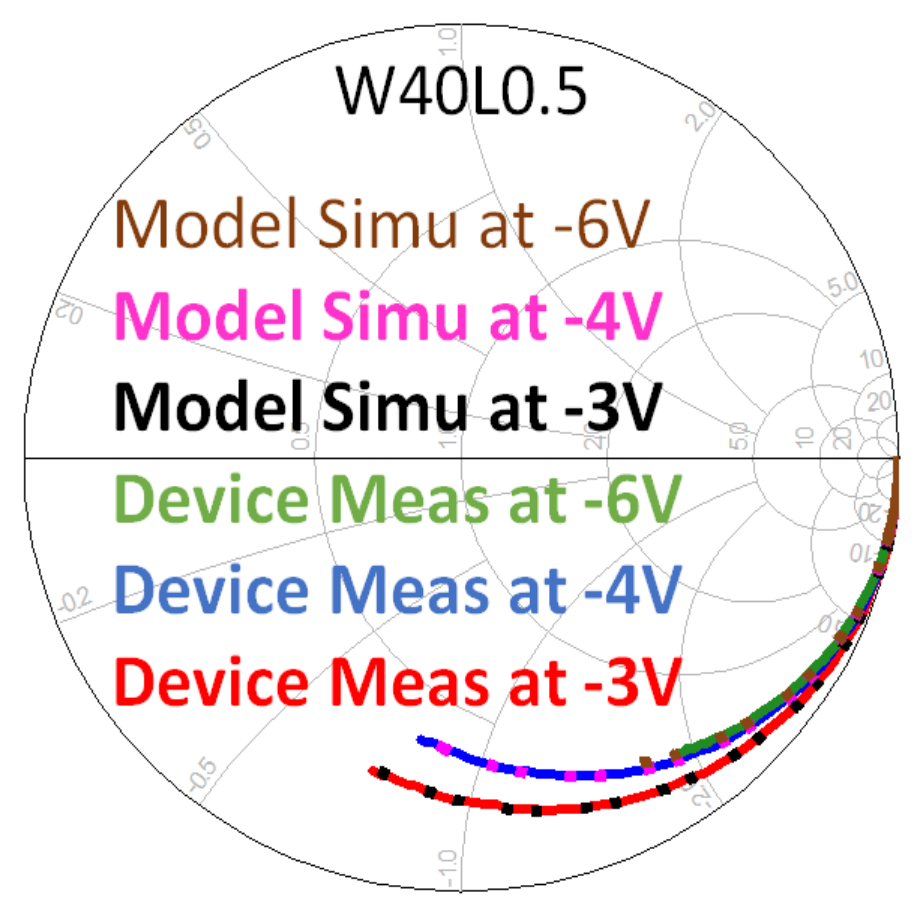

Figure III.18: Measured (lines) and Simulated (Dot) S11-parameter for G W4OLO.5

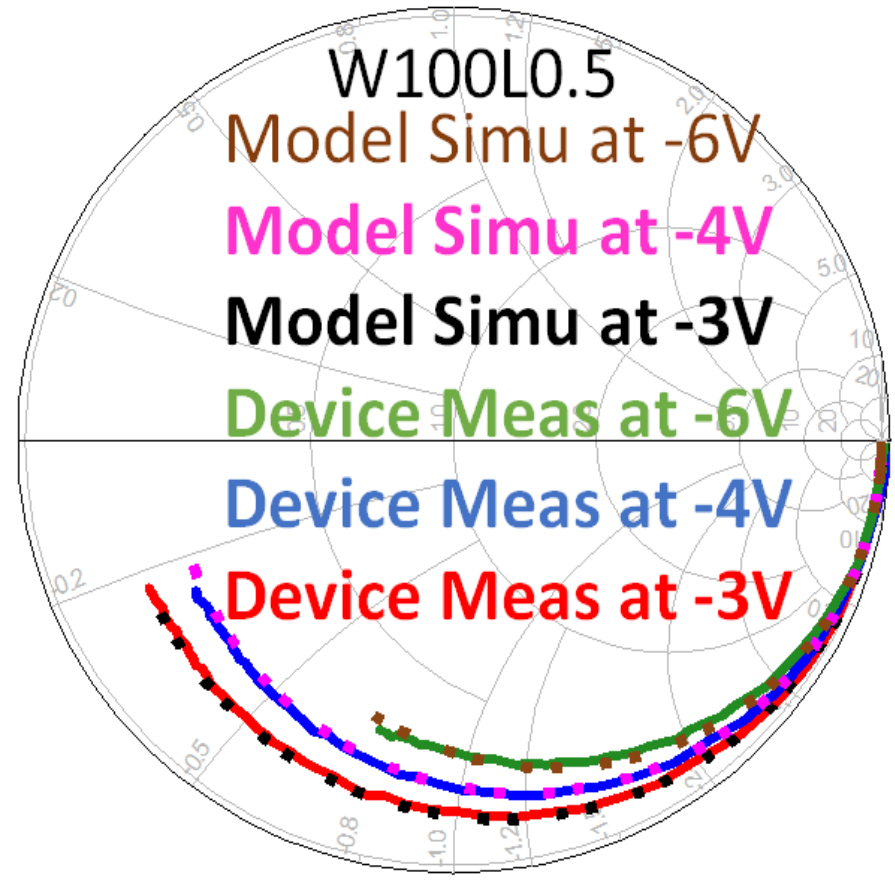

Figure III.19: Measured (lines) and Simulated (Dot) S11-parameter for W100L0.5 


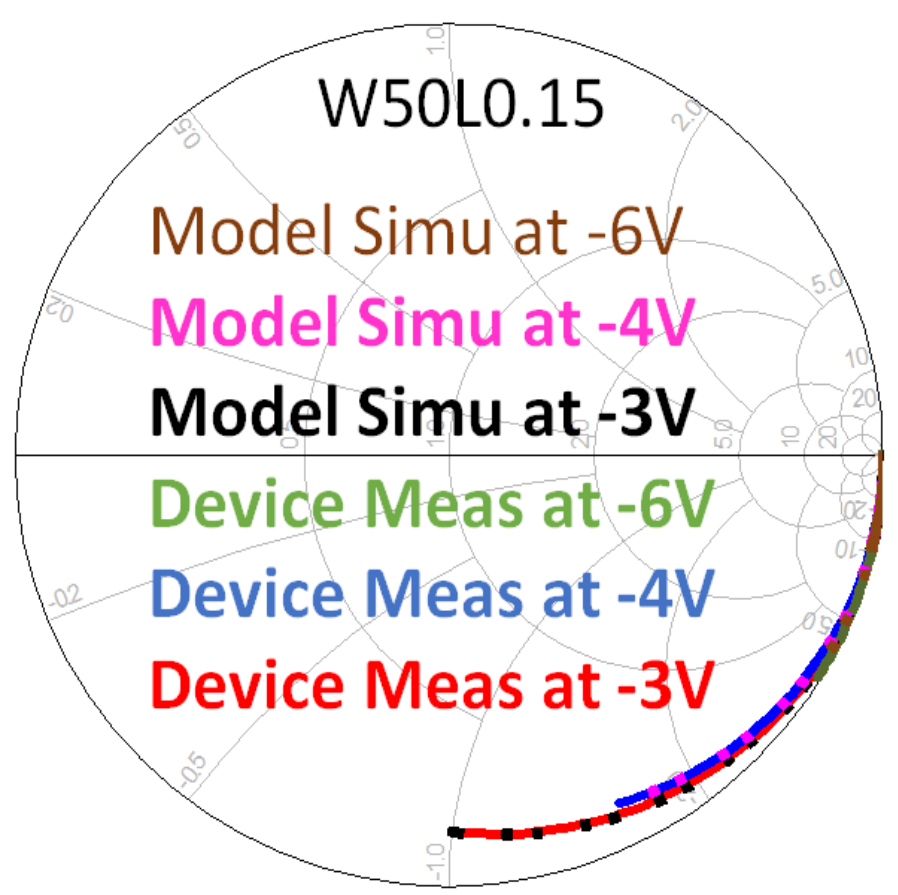

Figure III.20: Measured (lines) and Simulated (Dot) S11-parameter for W50L0.15

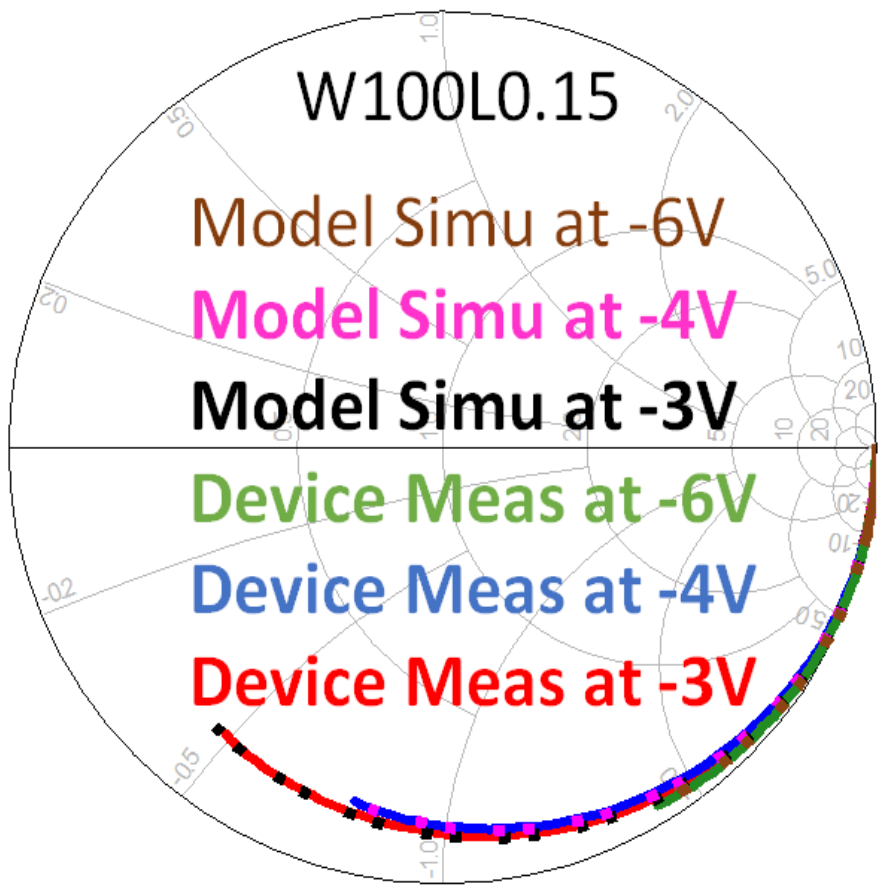

Figure III.21: Measured (lines) and Simulated (Dot) S11-parameter for W100L0.15 


\subsubsection{Analysis of Equivalent Capacitance}

At an operating frequency $f$, the capacitance values were extracted from the Y-parameters using the Equation (1). The obtained capacitance values versus frequency for one device, are shown in Figure III.22. At $\mathrm{V}_{\mathrm{G}}=-3 \mathrm{~V}$, it is seen that the capacitance values remain almost constant over a range of frequency up to $20 \mathrm{GHz}$, and then the capacitance behavior starts to become erratic due to high frequency parasitic element. This means that at very high frequency, the parasitic gate inductance, Lg, and other effects become self-resonant.

Equation (1)

$$
C e q=\frac{\operatorname{img}(\mathrm{Y}(1,1))}{2 . \pi \cdot \mathrm{f}}
$$

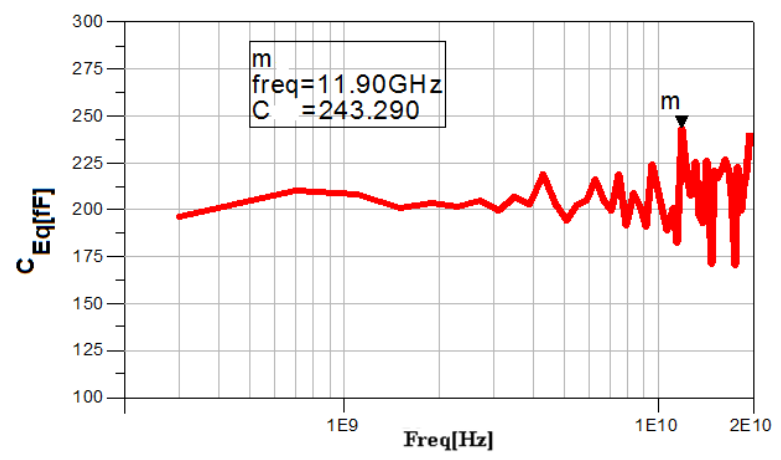

Figure III.22: $C_{E q}$ versus frequency for the W4OLO.5 device at $V_{G}=-3 \mathrm{~V}$

For both GaN500v1 and GaN150v0, the extracted capacitance values at frequency range up to $10 \mathrm{GHz}$ versus $\mathrm{V}_{\mathrm{G}}$ are shown in Figure III.23 and Figure III.24 (red curves). All $\mathrm{C}_{\mathrm{Eq}}$ versus $\mathrm{V}_{\mathrm{G}}$ curves exhibit the same general behavior, which can be separated into three regions: range (1) for $\mathrm{V}_{\mathrm{G}}$ above $-3.5 \mathrm{~V}$, range (2) for $-4.5 \mathrm{~V} \leq$ $\mathrm{V}_{\mathrm{G}} \leq-3.5 \mathrm{~V}$ and range (3) for $\mathrm{V}_{\mathrm{G}}<-4.5 \mathrm{~V}$. 

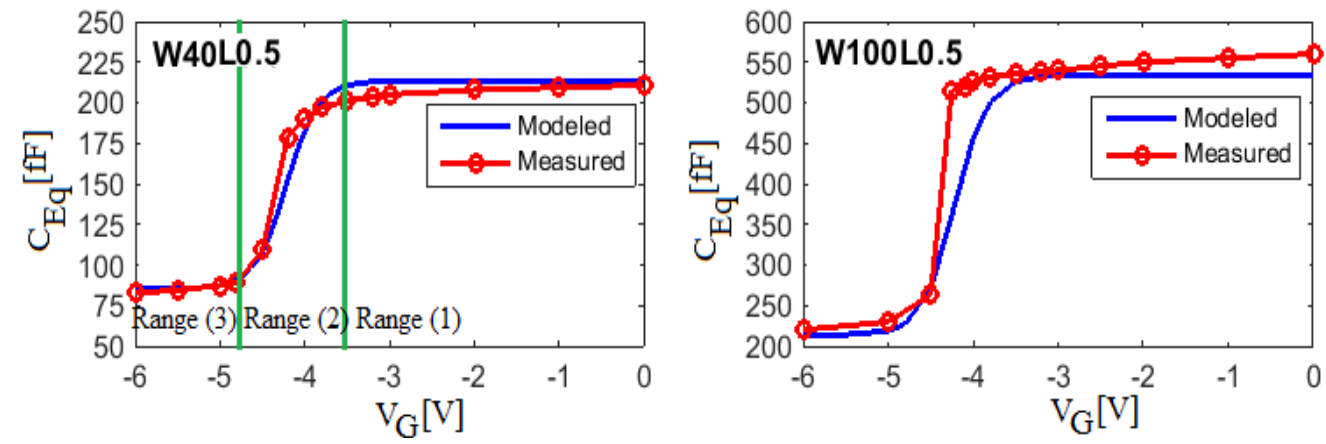

Figure III.23: Extracted and Simulated $C_{E q}$ versus $V_{G}$ for GaN500v1 Devices
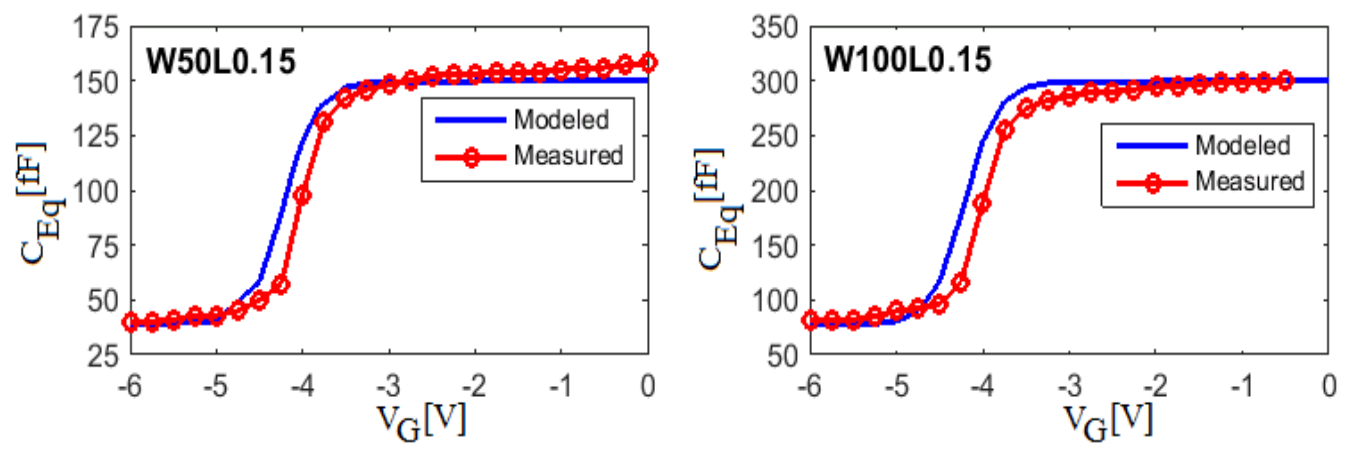

Figure III.24: Extracted and Simulated $C_{E q}$ versus $V_{G}$ for GaN150 Devices

For range (1) and range (3) corresponding to the device's $\mathrm{C}_{\mathrm{MAX}}$ and $\mathrm{C}_{\mathrm{MIN}}$, respectively, the capacitance values do not change. However, in range (2), the capacitance values change significantly, and rapidly over a small DC bias range from $-4.5 \mathrm{~V}$ to $-3.5 \mathrm{~V}$. Based on these observations, we conclude that the capacitance tuning range is limited, and the region of variable capacitance exists only over a small reverse voltage of $1 \mathrm{~V}$. The capacitance $\mathrm{C}_{\mathrm{Eq}}$ is given by the following equation:

Equation (2)

$$
\text { Ceq }=\frac{\varepsilon r \cdot A}{\mathrm{~d}} \quad \text { with } A=N . W . L
$$

Where, $\varepsilon r$ is the permittivity of the material, $A$ is the finger area, $\mathrm{d}$ is the distance between surface and 2DEG channel (or the depth of the depletion region), $N$ is the number of fingers, $W$ and $L$ are the width and the length of finger. From Equation (2), the capacitance value depends on d. In other words, when d varies, this 
leads to a change in the capacitance value. The maximum and the minimum $\mathrm{C}_{\mathrm{Eq}}$ are obtained when the $d$ reaches its minimum $\left(d_{\min }\right)$ and maximum $\left(d_{\max }\right)$, respectively. Furthermore, the capacitance value depends on the finger area. The variation in $d$ is controlled by $\mathrm{V}_{\mathrm{G}}$ and leading to the change in $\mathrm{C}_{\mathrm{Eq}}$ which could be summarized as follow:

- Range (1): Before the channel starts depleting; $\mathrm{C}_{\mathrm{Eq}}$ is at its maximum value where the depletion region is at $d_{\text {min }}$.

- Range (2): The channel starts depleting until it is completely depleted. The depleted region in the channel shrinks quickly, resulting in a rapid increase of $\mathrm{C}_{\mathrm{Eq}}$.

- Range (3): The channel is completely depleted; $\mathrm{C}_{\mathrm{Eq}}$ is at its minimum values where the depletion region is at $d_{\max }$.

In order to describe the nonlinear behavior of $\mathrm{C}_{\mathrm{Eq}}$ for both GaN500v1 and GaN150v0, analytical equations based on empirical coefficients are derived from the measurements. The exponential of tangent hyperbolic function yields an approximate analytical expression for $\mathrm{C}_{\mathrm{Eq}}$ versus $\mathrm{V}_{\mathrm{G}}$. It is seen that Equation (3) accurately describes the dependency of junction capacitance upon applied reverse bias, $V_{G}$, for all characterized devices.

Equation (3)

$$
\text { Ceq } \left.=60 \frac{A}{M}\left(1+\frac{1}{M}\right)\left(e^{\tanh [2.5 V g+11]}\right)+k\right)
$$

The parameters $M$ and $K$ can be adjusted to properly fit the $\mathrm{C}_{\mathrm{Eq}}$ equation to the measured characteristics of an arbitrary varactor size. According to Matlab simulations, the values of $M$ and $K$ can be fixed, respectively, as 40 and 1.2 for GaN500v1 devices and as 20 and 0.45 for GaN150 devices. The modeled curves are plotted in Figure III.23 and Figure III.24 (blue lines). All of these equations are dependent only on finger geometry (number, width and length of fingers) and on $\mathrm{V}_{\mathrm{G}}$. Equation (3) illustrates the behavior of $\mathrm{C}_{\mathrm{Eq}}$ for GaN500v1 and GaN150v0 devices. 
This equation describes the experimental results adequately under small signal excitation.

\subsubsection{Analysis of Equivalent Series resistance}

The equivalent series resistor contains all the resistors $\left(R_{\text {cst }}, R_{\text {sheet }}, R_{\text {dep }}, R_{g}\right.$, $R_{s}$ and $R_{d}$ ) presented in Figure III.15. In range (1), before the channel starts being depleted, there is no depletion resistor $\left(R_{\text {dep }}=0\right)$, leading to have a constant equivalent series resistor. In range (2) when the channel starts depleting, the $R_{\text {dep }}$ becomes important and increases until the channel is close to be fully depleted resulting on $R_{E q}$ values. Over range (3), when the channel is fully depleted, $R_{\text {dep }}$ goes to 0 resulting in a constant equivalent series resistor $R_{E q}$. In Figure III.25 and Figure III.26, $R_{E q}$ versus $V_{G}$ is plotted. $R_{E q}$ (red curves) is determined by extracting the real part of $\mathrm{Z}$ in the equivalent circuit model.
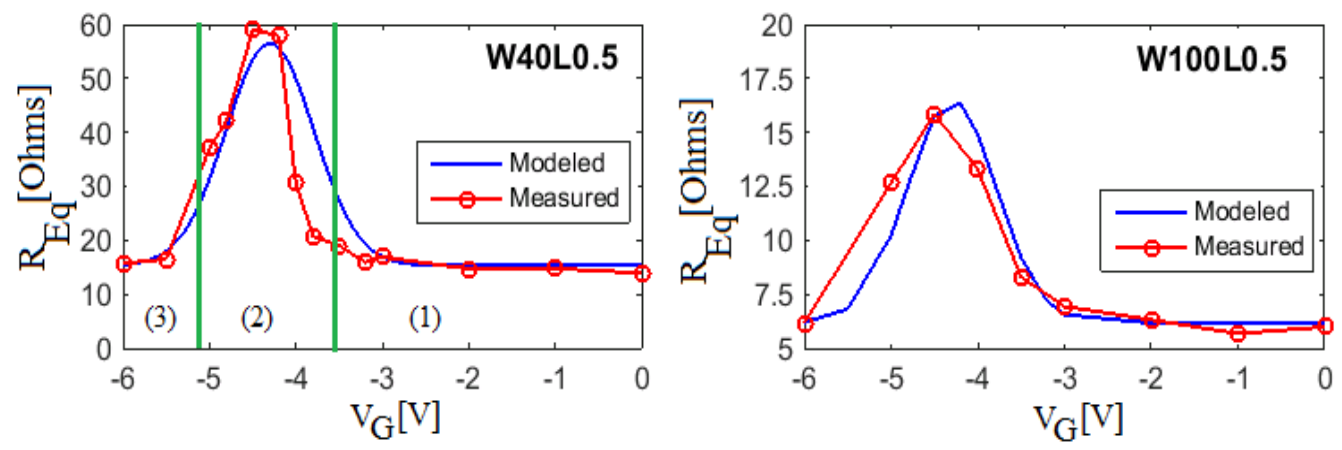

Figure III.25: Exctacted and Simulated $R_{E q}$ versus $V_{G}$ at $2.5 \mathrm{GHz}$ for GaN500v1
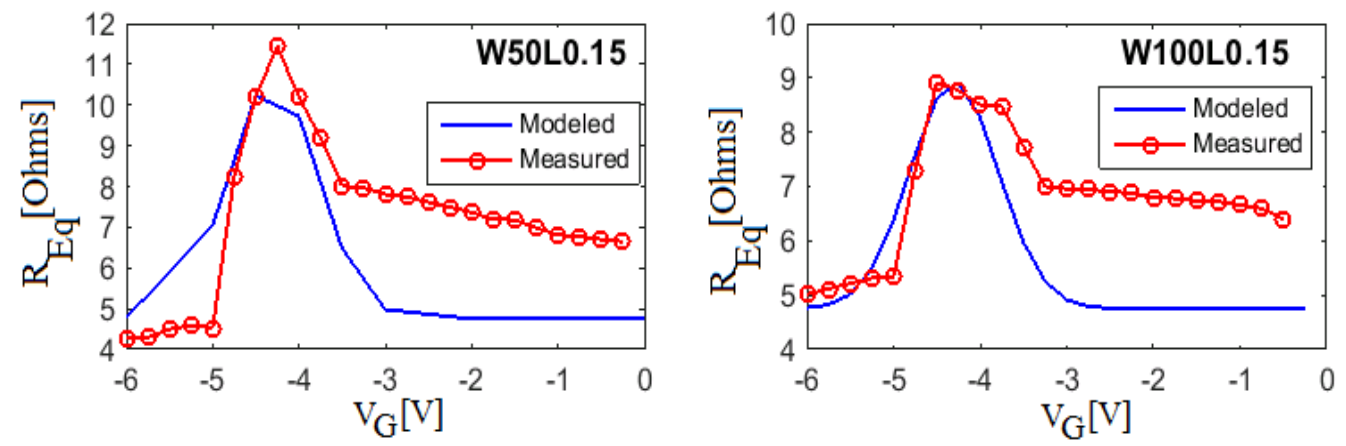

Figure III.26: Extracted and Simulated $R_{E q}$ versus $V_{G}$ at $2.5 \mathrm{GHz}$ for GaN150vO 
The nonlinear behavior of $\mathrm{R}_{\mathrm{Eq}}$ for both GaN500v1 and GaN150v0 can be described by analytical equations based on empirical. The $R_{E q}-V_{G}$ characteristics is a Gaussian form, as deduced from Figure III.25 and Figure III.26 (red dotted lines). Thus the $\mathrm{R}_{\mathrm{Eq}}-\mathrm{V}_{\mathrm{G}}$ characteristics are taken to be:

Equation (4)

$$
\text { ReqGaN500 }=\frac{44}{\mathrm{~A}}\left[\left(\frac{1}{\sqrt{\frac{W \pi}{\mathrm{Y}} 8 \cdot 10^{-5}}}\right)\left(e^{-2(V g+4.35)^{2}}\right)+Y\right]
$$

Equation (5)

$$
\operatorname{ReqGaN} 150=\left[\left(\frac{1}{6 \sqrt{\frac{A \pi}{\mathrm{Y}} 8 \cdot 10^{-5}}}\right)\left(e^{-2(V g+4.35)^{2}}\right)+Y\right]
$$

Where $\mathrm{A}$ is the finger area. The parameter $Y$ can be adjusted to properly fit the $R_{E q}$ equation to the measured characteristics of an arbitrary varactor size. According to Matlab simulations, the value $Y$ can be fixed to 14 for GaN500v1 devices and as 4.75 for GaN150 devices. All of these equations are dependent only on finger geometry (number, width and length of fingers) and on $\mathrm{V}_{\mathrm{G}}$. Equation (4) describes $\mathrm{R}_{\mathrm{Eq}}$ for GaN500v1 devices, while Equation (5) describes $R_{E q}$ for GaN150v0 devices. These equations describe the experimental results adequately under small signal excitation. Due to these resistance values, a part of the RF available power is consumed by the devices and losses will occur in the $\mathrm{RF}$ signal. More $\mathrm{R}_{\mathrm{Eq}}$ is high more losses are high. A good agreement is obtained, except for $R_{E q}$ in range (1) of the GaN150v0 devices. A slightly more complicated model would be required here to capture the higher measured $\mathrm{R}_{\mathrm{Eq}}$.

\subsubsection{Analysis of Q-Factor}

In Figure III.27 and Figure III.28, the Q factor is plotted versus frequency. Referring to the equivalent model presented in Figure III.17, the $\mathrm{Q}$ factor can be evaluated by the following equation.

Equation (6) 


$$
Q=\frac{\operatorname{img}(Z(1,1))}{\operatorname{real}(Z(1,1))}=\frac{1}{(2 \cdot \pi \cdot f \cdot \text { Ceq.Req })}
$$
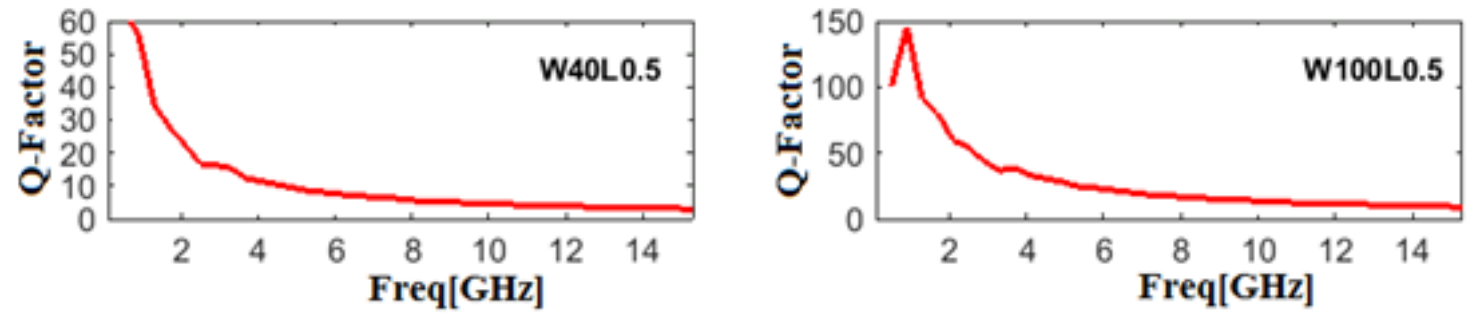

Figure III.27: Extracted $Q$ factor versus Frequency at $V_{G}=-3.5 \mathrm{~V}$ for GaN500v1
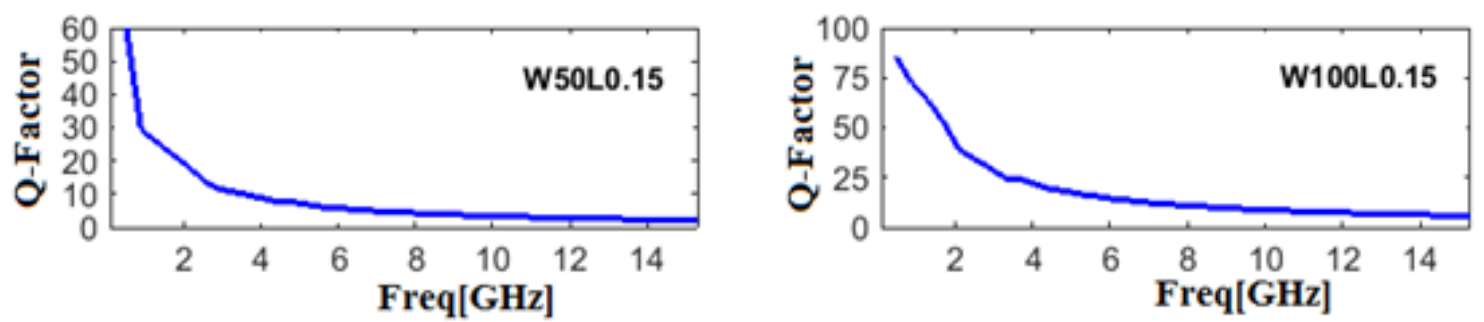

Figure III.28: Extracted $Q$ factor versus Frequency at $V_{G}=-3.5 \mathrm{~V}$ for GaN150vO

The $\mathrm{Q}$ factor decreases with frequency. It is notable that a minimum $\mathrm{Q}$ of $\sim 25$ and $\sim 50$ was achieved over a range of frequency below $2 \mathrm{GHz}$, respectively, for GaN500v1 and GaN150v0. Another most common used figure of merit for varactor is the cut-off frequency. The cut-off frequency is defined as the frequency at which the capacitive reactance is equal to the series resistance. Note that the cut-off frequency is also a function of voltage and that it achieves its maximum value at VB. This FOM indicates that the maximum operating frequency of the circuit is dependent on the series resistance and the equivalent capacitance. FOM should be as high as possible, which can be mathematically given as:

Equation (7)

$$
\text { Fcut_off }=\frac{1}{(2 \cdot \pi \cdot \text { Ceq.Req) }}
$$


At $\mathrm{f}=2.5 \mathrm{GHz}$, maximum values of $164.9 \mathrm{GHz}$ and of $419.2 \mathrm{GHz}$ were achieved, respectively, for W40L0.5 and W100L0.5 at $\mathrm{V}_{\mathrm{G}}=-5 \mathrm{~V}$. While for W50L0.15 and W100L0.15, the maximum obtained values are $770.6 \mathrm{GHz}$ and $335.9 \mathrm{GHz}$, respectively. These obtained values serve as figure of merit to compare with other varactor diode in terms of frequency operation capability. A comparison between the two GaN processes in terms of capacitor $\mathrm{C}_{\mathrm{Eq}}$ is presented in Table III.3.

Table III.3: Comparison of capacitance performances between GaN500 and GaN150

\begin{tabular}{|c|c|c|}
\hline & GaN500v1 & GaN150v0 \\
\hline Capacitance & $2.7 f F / u m$ & $1.5 f F / u m$ \\
\hline$C_{\text {MAX }} / C_{\text {MIN }}$ & 2.4 & 3.7 \\
\hline Tuning Voltage & -4.5 V to $-3.5 \mathrm{~V}$ & -4.5 V to $-3.5 \mathrm{~V}$ \\
\hline
\end{tabular}

\section{GaN500v2 Varactor Devices}

To conduct this second varactors modeling study, many samples with different sizes have been fabricated with the GaN500v2 process but only three of them are presented here (see Table III.4). The presented devices are W50L0.5, W100L0.5 and W200L0.5 (different fingers width).

Table III.4 : Presented GaN500v2 Devices Information

\begin{tabular}{|c|c|c|c|c|}
\hline $\begin{array}{c}\text { Device } \\
\text { Terminology }\end{array}$ & $\begin{array}{c}\text { Number of } \\
\text { Fingers }\end{array}$ & $\begin{array}{c}\text { Fingers } \\
\text { Width }\end{array}$ & $\begin{array}{c}\text { Fingers } \\
\text { Length }\end{array}$ & $\begin{array}{c}\text { Process } \\
\text { Terminology }\end{array}$ \\
\hline W50L0.5 & $\mathbf{2}$ & $\mathbf{5 0} \boldsymbol{\mu m}$ & $\mathbf{0 . 5 0} \boldsymbol{\mu m}$ & GaN500v2 \\
\hline W100L0.5 & $\mathbf{2}$ & $\mathbf{1 0 0} \boldsymbol{\mu m}$ & $\mathbf{0 . 5 0} \boldsymbol{\mu m}$ & GaN500v2 \\
\hline W200L0.5 & $\mathbf{2}$ & $\mathbf{2 0 0} \boldsymbol{\mu m}$ & $\mathbf{0 . 5 0} \boldsymbol{\mu m}$ & GaN500v2 \\
\hline
\end{tabular}

\subsection{Small Signal Measurement}

The on-wafer RF measurements were performed with an Agilent VNA Network Analyzer over a frequency range of 0.1-20 GHz with a fixed RF power of $15 \mathrm{dBm}$, while the control voltage at the gate of varactors, $\mathrm{V}_{\mathrm{G}}$, was swept from $0 \mathrm{~V}$ to $-10 \mathrm{~V}$ with a step of $-0.1 \mathrm{~V}$. Because it was observed that from $0 \mathrm{~V}$ to $-3 \mathrm{~V}$, the measured results are similar and also from $-5 \mathrm{~V}$ to $-10 \mathrm{~V}$ measured results are similar. So only measurements from $-2 \mathrm{~V}$ to $-6 \mathrm{~V}$ will be treated and analysed here. As can be 
seen in Figure III.29, Figure III.30 and Figure III.31, a good match between the experimental results and the simulation model results is generally achieved. For W100L0.5 and W200L0.5, agreement is nearly perfect, while for W50L0.5 there are some issues with this device at high frequencies. Here some parasitic inductances are not captured in the simple proposed equivalent model. As detailed previously, there are three main ranges that describe the change in the parameters of the varactor model. Range (1) and range (3) corresponding to the device's $\mathrm{C}_{\mathrm{MAX}}$ and $\mathrm{C}_{\mathrm{MIN}}$, respectively, while, range (2) corresponding to the tuning capacitance range. Table III.5 summarizes the parameter values of the equivalent model at three bias voltage, such as, $\mathrm{V}_{\mathrm{G}}$ of $-3 \mathrm{~V}$ (in range 1 ), $-4 \mathrm{~V}$ (in range 2) and $-6 \mathrm{~V}$ (in range 3 ).

Table III.5: Parameters values of the equivalent circuit

\begin{tabular}{|c|c|c|c|c|}
\hline Device & $\mathrm{V}_{\mathrm{G}}(\mathrm{V})$ & $\mathrm{L}_{\mathrm{para}}(\mathrm{pH})$ & $\mathrm{R}_{\mathrm{Eq}}(\Omega)$ & $\mathrm{C}_{\mathrm{Eq}}(\mathrm{fF})$ \\
\hline W50L0.5 & -3 & 5 & 7.9 & 466 \\
\cline { 2 - 5 } & -4 & 5 & 11 & 375 \\
\cline { 2 - 5 } & -6 & 5 & 9.5 & 279 \\
\hline \multirow{3}{*}{ W100L0.5 } & -3 & 16 & 5.3 & 870 \\
\cline { 2 - 5 } & -4 & 16 & 7.35 & 637 \\
\cline { 2 - 5 } & -6 & 16 & 6.5 & 501 \\
\hline W200L0.5 & -3 & 100 & 4.7 & 1810 \\
\cline { 2 - 5 } & -4 & 100 & 5.85 & 1403 \\
\cline { 2 - 5 } & -6 & 100 & 5.2 & 1016 \\
\hline
\end{tabular}




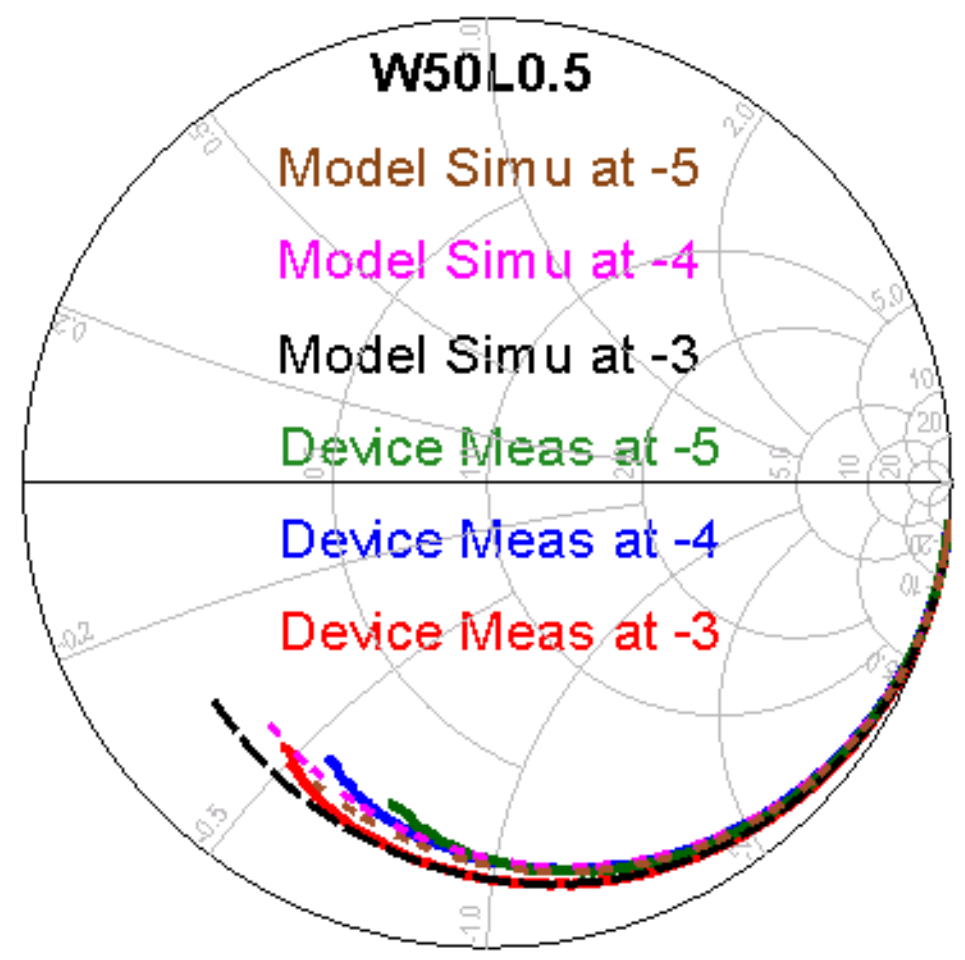

Figure III.29: Measured (lines) and Simulated (Dot) S11-parameter for W50L0.5

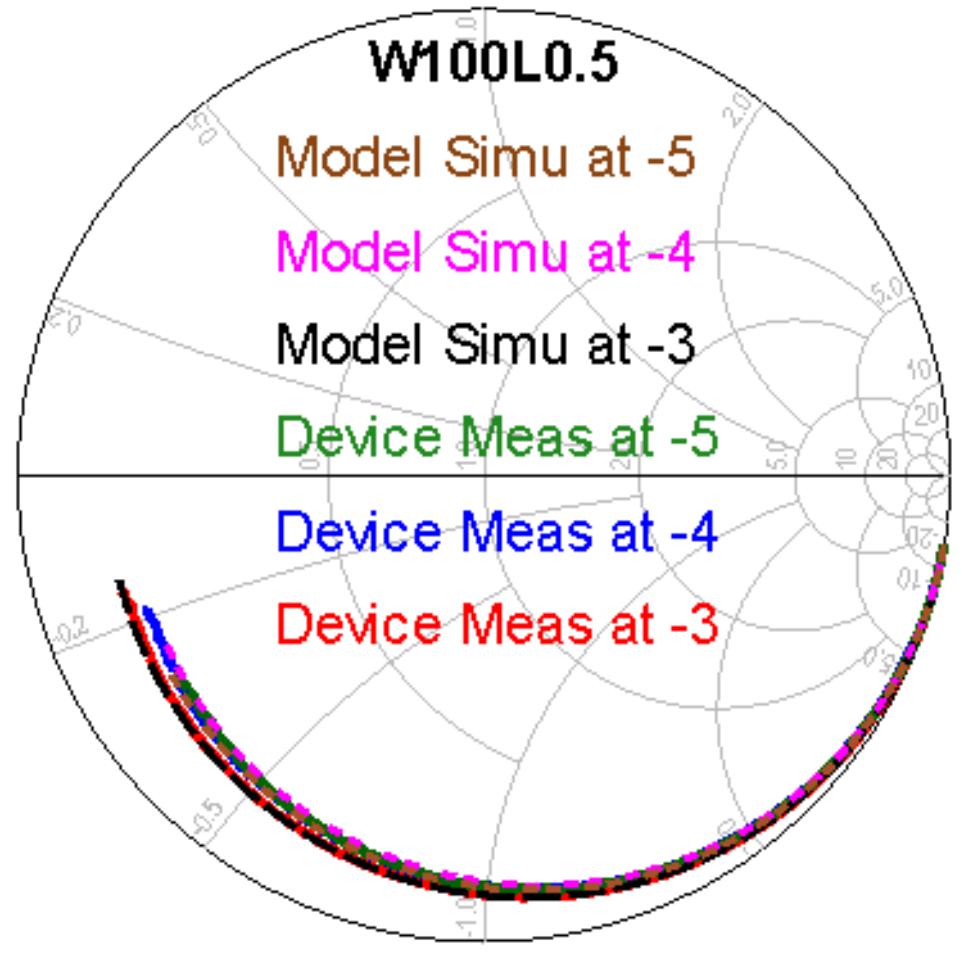

Figure III.30: Measured (lines) and Simulated (Dot) S11-parameter for G W100L0.5 


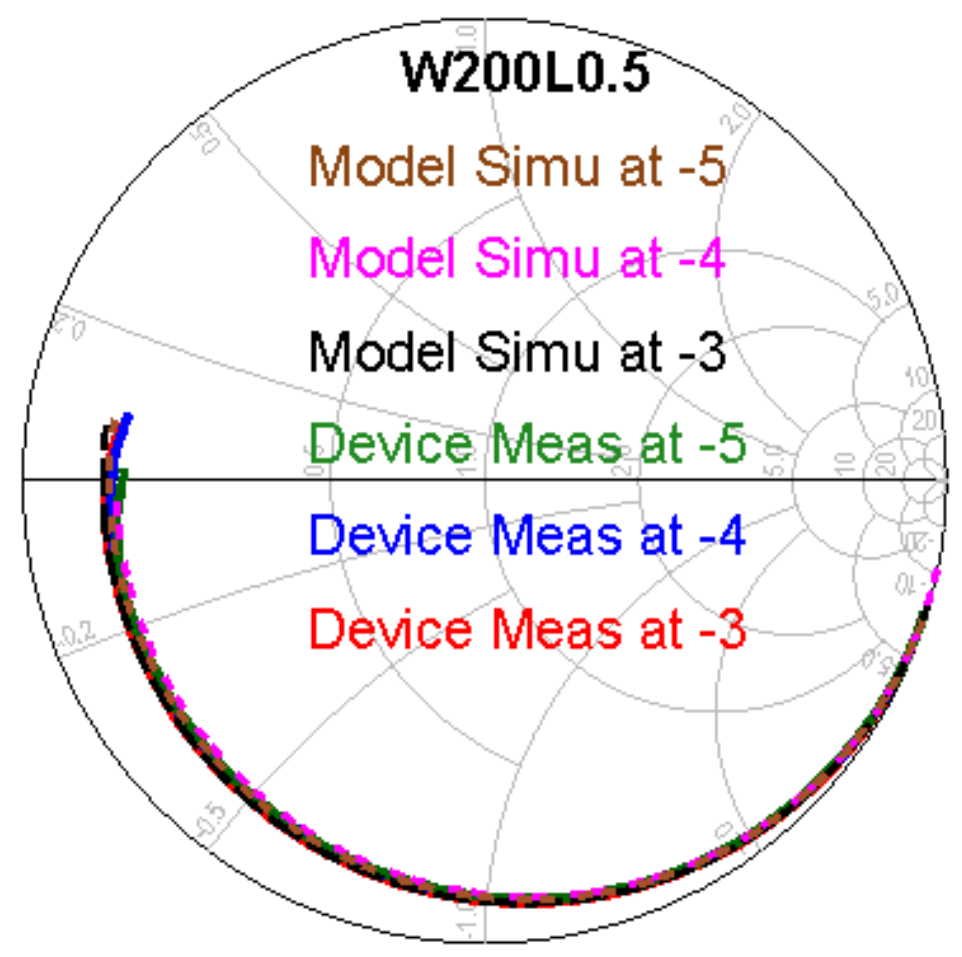

Figure III.31: Measured (lines) and Simulated (Dot) S11-parameter for G W200L0.5

\subsubsection{Analysis of Equivalent Capacitance}

The same analysis drawn before for GaN500v1 devices will be also used here to discuss and develop the extracted equivalent capacitance values for GaN500v2 devices.

As previously mentioned, the capacitance values were extracted from the Y-parameters using Equation (1). The obtained capacitance values versus frequency for one device (W50L0.5), are shown in Figure III.32. At $\mathrm{V}_{\mathrm{G}}=-3 \mathrm{~V}$, it is seen that the capacitance values remain almost constant over a range of frequency up to $8 \mathrm{GHz}$, and then the capacitance starts to decrease due to high frequency parasitic elements. This means that at very high frequency, the parasitic gate inductance, Lg, and other effects become self-resonant. 


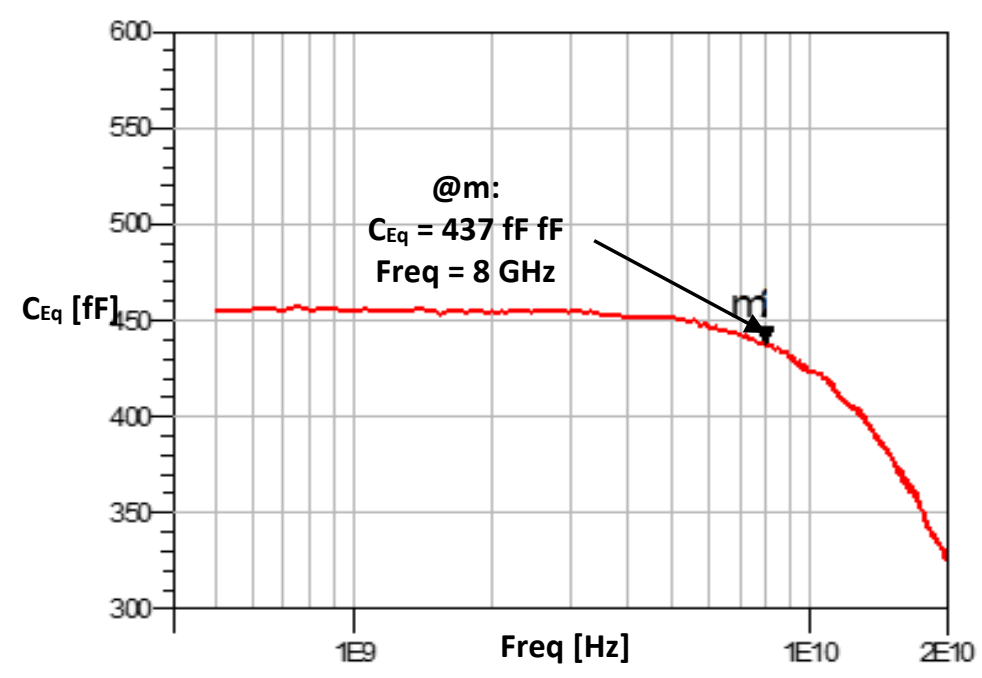

Figure III.32: $C_{E q}$ versus frequency for the W50L0.5 device at $V G=-3 \mathrm{~V}$

For all devices W50L0.5, W100L0.5 and W200L0.5, the extracted and the modeled capacitance values versus $\mathrm{V}_{\mathrm{G}}$ are, respectively, shown in Figure III.33, Figure III.34, and Figure III.35. These extracted values are valid over a frequency range frequency up to $8 \mathrm{GHz}$ where $\mathrm{C}_{\mathrm{Eq}}$ starts drops versus frequency (see Figure III.32). As can be seen from these three figures, it should be mentioned that poorer agreement for large $\mathrm{W}$ (larger device) is a model optimization issue. This means that a slightly more complicated model would be required here to capture the all the parasitic elements for larger devices.

It is noted that the Equation (3) presented before for GaN500v1 devices is used here to describe the behavior of $\mathrm{C}_{\mathrm{Eq}}$ versus $\mathrm{V}_{\mathrm{G}}$. It valids the general use of this proposed equation for GaN-based varactor devices. Only the parameters $M$ and $K$ were adjusted to properly fit the $\mathrm{C}_{\mathrm{Eq}}$ equation to the measured characteristics of an arbitrary varactor size. For GaN500v2, the values of $M$ and $K$ were re-optimized to fit the model with the measurements, respectively, are 66 and 2.4. As mentioned before, for GaN500v1 devices, this equation is dependent only on sample size (number, width and length of fingers) and on $\mathrm{V}_{\mathrm{G}}$. As can be seen, $\mathrm{C}_{\mathrm{Eq}}$ versus $\mathrm{V}_{\mathrm{G}}$ characteristics exhibit the same general behavior observed for GaN500v1 devices, which is separated into three regions as discussed before. 
The maximum value of capacitance (before the channel starts depleting) is observed for $-3.4 \mathrm{~V} \leq \mathrm{V}_{\mathrm{G}} \leq 0 \mathrm{~V}$ (range 1) and values of $466 \mathrm{fF}, 870 \mathrm{fF}$ and $1810 \mathrm{fF}$ were extracted, respectively, for W50L0.5, W100L0.5 and W200L0.5 designs. While the minimum value (the channel is completely depleted) is observed for $-10 \mathrm{~V} \leq \mathrm{V}_{\mathrm{G}} \leq-4.5$ $\mathrm{V}$ (range 3) and values of $278 \mathrm{fF}, 501 \mathrm{fF}$ and $1016 \mathrm{fF}$ were extracted for, respectively, W50L0.5, W100L0.5 and W200L0.5 designs. As a result, $\mathrm{C}_{\mathrm{MAX}} / \mathrm{C}_{\mathrm{MIN}}$ ratio about 1.7 is achieved for all devices. In addition, it was observed that $\mathrm{C}_{\mathrm{MAX}}$ and $\mathrm{C}_{\mathrm{MIN}}$ values remain almost constant over a range of frequency up to almost $8 \mathrm{GHz}$ and then starts to drop significantly due to high frequency parasitics. The $-3.4 \mathrm{~V}$ represents the pinched of voltage of this device where the channel starts depleting. As highlighted before for GaN500v1 devices, in range (2), the capacitance values change significantly and rapidly over a small DC bias range of $1 \mathrm{~V}$. This behavior is also observed for the devices using the GaN500v2.

Because we are essentially focused on the range (2) where capacitance values change significantly, the $\mathrm{x}$-axis of all $\mathrm{C}-\mathrm{V}$ plots is delimited from $-2 \mathrm{~V}$ to $-6 \mathrm{~V}$. Values from $0 \mathrm{~V}$ to -2 corresponding to $\mathrm{C}_{\mathrm{MAX}}$ are stable as well as ones from $-6 \mathrm{~V}$ to $10 \mathrm{~V}$ corresponding to $\mathrm{C}_{\mathrm{MIN}}$.

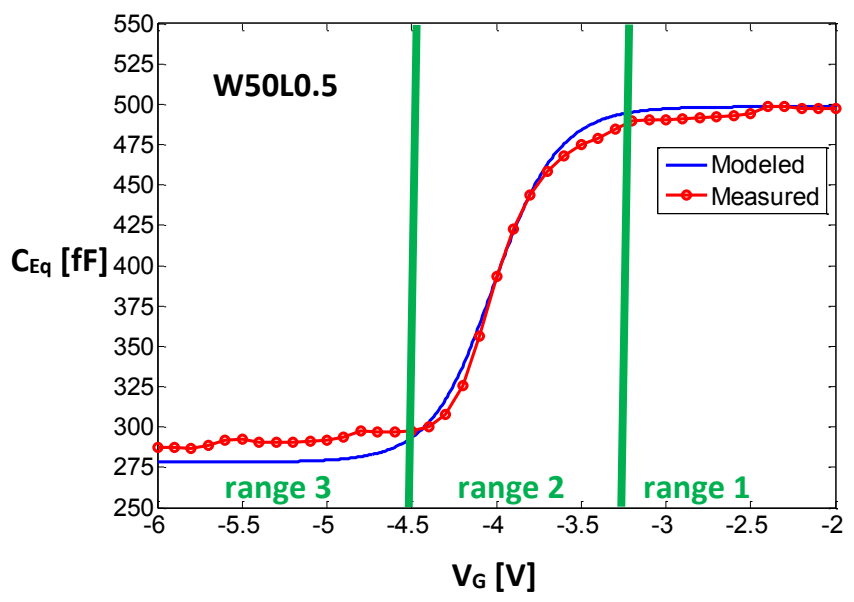

Figure III.33: Extracted and Simulated $C_{E q}$ versus $V_{G}$ for W50L0.5 


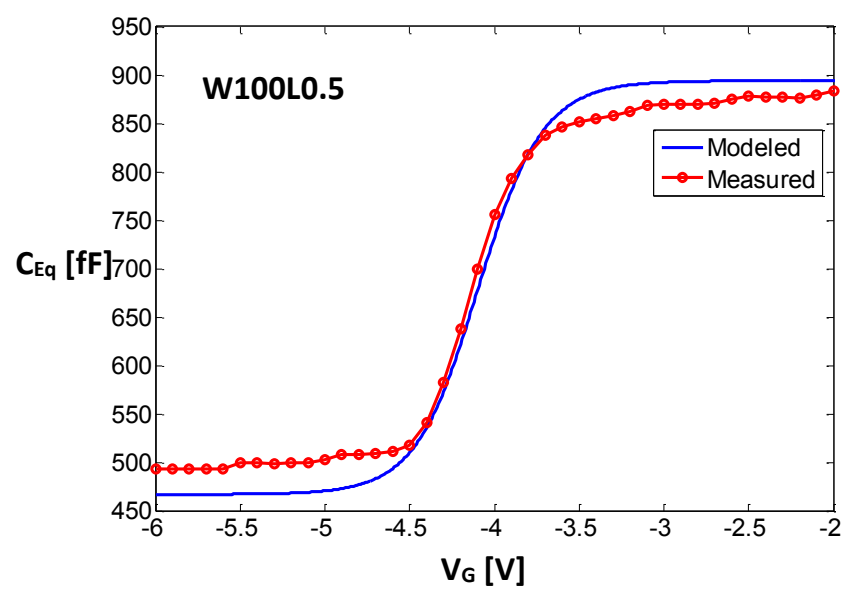

Figure III.34: Extracted and Simulated $C_{E q}$ versus $V_{G}$ for W100L0.5

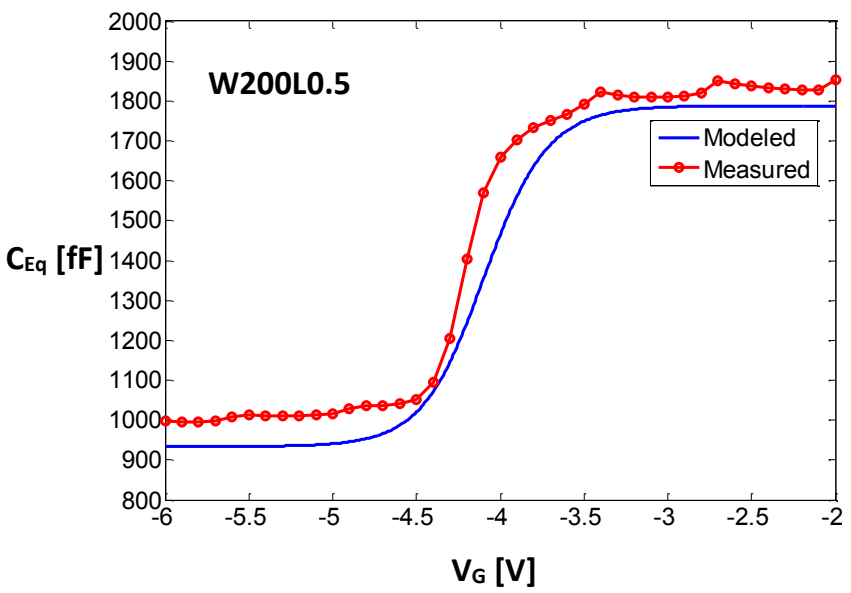

Figure III.35: Extracted and Simulated $C_{E q}$ versus $V_{G}$ for W200L0.5

By comparing equivalent capacitance values extracted from GaN500v1 samples and from GaN500v2 samples, it was revealed that the achieved capacitance values from a device size manufactured with GaN500v2 are at least twice larger than those achieved with the same device size fabricated with GaN500v1 process. However, the overall calculated $\mathrm{C}_{\mathrm{MAX}} / \mathrm{C}_{\mathrm{MIN}}$ ratios for GaN500v2 devices are smaller $(\sim 1.7)$ than ones calculated from GaN500V1 devices ( 2.4). This significant change in equivalent capacitance values is essentially due to the additional field plate layer added in GaN500v2. As discussed in the GaN500 design manual provided by NRC, it should be pointed out that all devices included in GaN500v1, do not employ field plate 
designs. However, the shape of the gates, using GaN500v1, results in better frequency performance, but slightly lower breakdown voltage compared to GaN500v2. For GaN500v1, values of $27 \mathrm{GHz}$ and $75 \mathrm{GHz}$ were reported by NRC, respectively, for $\mathrm{f}_{\mathrm{T}}$ and for $\mathrm{f}_{\mathrm{max}}$. The process is suitable for $20 \mathrm{~V}$ maximum drain voltage bias. While for GaN500v2 process, a field plated design is included in this version, and devices exhibit much lower gate leakage than in GaN500v1. However, RF performance frequency decreased; a $\mathrm{f}_{\mathrm{T}}$ of $12 \mathrm{GHz}$ and $\mathrm{f}_{\max }$ of 24 values were measured and reported for this version. The process version is suitable for $40 \mathrm{~V}$ maximum drain voltage, and yields power levels of $\sim 5 \mathrm{~W} / \mathrm{mm}$. We assume that this additional field plate layer is the main parameter behind the significant increase in the equivalent capacitance values.

\subsubsection{Analysis of Equivalent Series Resistance}

The equivalent series resistor extracted from measurement versus bias voltage is depicted in Figure III.36 for all studied devices. This series resistor is not impacted by the frequency, so these extracted values are valid at all frequencies. Here the same general behaviour observed before for GaN500v1 is also noticed for the devices fabricated with GaN500v2, expect that the values of the series resistor are significantly different from a process version to another one.

Unlike the GaN500v1 devices where an analytic expression was introduced to describe the behavior of $\mathrm{R}_{\mathrm{Eq}}$ versus $\mathrm{V}_{\mathrm{G}}$, here for GaN500v2 devices, as can be clearly seen the equivalent series resistor values do not change significantly and this is observed for all devices, so a mean value can be adopted as a fixed value to describe the $R_{E q}$ for a specific device size. In addition, it was observed than more the device size increases more the $R_{E q}$ decreases. The maximum peak value of $R_{E q}$ is observed on range (2) when the channel starts depleting because the depletion resistor $\left(R_{\text {dep }}\right)$ becomes slightly important and increases until the channel is close to be fully depleted. This behavior has been also observed for GaN500v1 process' devices. It should be pointed out the overall series resistor values extracted from GaN500v2 
devices are smaller than ones found with the previous process version (GaN500v1). In our point of view, this significant drop in series resistor can be due to the development occurred out on the stack of epitaxial layers and masks of the process to reduce the dynamic on-resistance and the Ohmic contact resistance that are constituting the series equivalent resistor $\left(\mathrm{R}_{\mathrm{Eq}}\right)$.

As mentioned in chapter II, the trapping electrons phenomena in AlGaN/GaN HEMTs structure is directly reducing the sheet electron density in the channel, leading to increase dynamic Ron and provoking drain current dispersion. Moreover, many efforts for high performance AlGaN/GaN HEMTs have been made to suppress this current dispersion issue and increased Ron, which are directly related to modification of surface/interface states in AlGaN/GaN heterostructures. These modifications are surface passivation [60], a thin AlN barrier layer in AlGaN/GaN interface [61], application of a field plate [62], surface treatments with chemicals or plasma [63], and postgate-annealing [64]. On the other hand, in spite of the rapid development and the studies of GaN-based HFETs, achieving a low Ohmic contact resistance value still one important issue concerning these devices [65]-[66] and has a great effect on the output signal during device operation. Low Ohmic contact resistance is crucial to realization of good $\mathrm{RF}$ and power performances of AlGaN/GaN HEMTs heterojunction devices. As a matter of fact, in terms of power consumption, high Ohmic contact resistance values cause high electrical power losses and increase self-heating problem. For example, if the contact resistor is decreased from $10^{-5}$ to $10^{-6} \Omega \mathrm{cm}^{2}$, a reduction of $60 \%$ of the power consumption could be achieved for devices with breakdown voltage of $600 \mathrm{~V}$ [67].

Due to the restricted information, we could not have information on how NRC group researchers have improved GaN500v2 process to reduce the Ohmic contact resistance or/and Ron. However, it is reported, by NRC in design manual of GaN500v2 [31], that a field plate is applied to all devices fabricated with GaN500v2. 


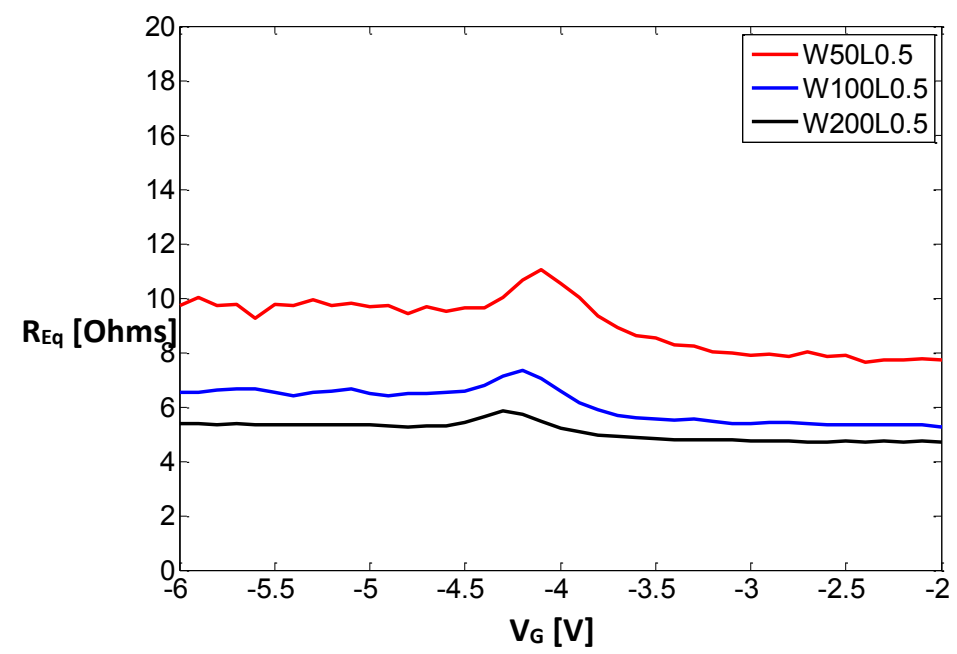

Figure III.36: Extracted $R_{E q}$ versus $V_{G}$ for GaN500v2 Devices

A comparison between the two GaN500 processes, in terms of model circuit parameters $\left(\mathrm{C}_{\mathrm{Eq}}, \mathrm{R}_{\mathrm{Eq}}\right.$ and $\left.\mathrm{L}_{\text {para }}\right)$ is presented in Table III.6.

Table III.6: Model Circuit Parameters Values

\begin{tabular}{|c|c|c|c|c|c|c|c|}
\hline \multirow[t]{2}{*}{ Device } & \multirow[t]{2}{*}{$\mathrm{V}_{\mathrm{G}}(\mathrm{V})$} & \multicolumn{3}{|c|}{ GaN500v1 Process } & \multicolumn{3}{|c|}{ GaN500v2 Process } \\
\hline & & Lpara $(\mathrm{pH})$ & $\operatorname{REq}_{\mathrm{Eq}}(\Omega)$ & $C_{E q}(f F)$ & Lpara $(\mathrm{pH})$ & $\operatorname{Req}_{\mathrm{Eq}}(\Omega)$ & $C_{E q}(f F)$ \\
\hline \multirow[t]{3}{*}{ W50L0.5 } & -3 & 25 & 17 & 205 & 5 & 7.7 & 466 \\
\hline & -4 & 25 & 50 & 190.7 & 5 & 9.95 & 375 \\
\hline & -6 & 25 & 15.5 & 82.7 & 5 & 9.2 & 279 \\
\hline \multirow[t]{3}{*}{ W40L0.5 } & -3 & 30 & 6.9 & 533 & 16 & 5.2 & 870 \\
\hline & -4 & 30 & 13.3 & 439 & 16 & 6.9 & 637 \\
\hline & -6 & 30 & 6.16 & 220 & 16 & 6.2 & 501 \\
\hline \multirow[t]{3}{*}{ W200L0.5 } & -3 & & & & 100 & 4.7 & 1810 \\
\hline & -4 & & & & 100 & 5.6 & 1403 \\
\hline & -6 & & & & 100 & 5.2 & 1016 \\
\hline
\end{tabular}




\subsection{Large Signal Measurements}

As we have just presented, the small signal analysis leading to propose a circuit model has been discussed. In addition, analytical expressions describing the general behavior of $\mathrm{C}_{\mathrm{EQ}}$ and $\mathrm{R}_{\mathrm{Eq}}$ for several $\mathrm{DC}$ bias were introduced. Following our original modeling and analysis of GaN-based varactor diodes, by using only a standard GaN HEMTs process; here, the effect of larger power signal is studied and presented to complete the analysis of $\mathrm{GaN}$ varactor small and large signal modeling. Unlike to GaN500v1 devices, the newly developed version of GaN500v2 is used for manufacturing the samples which have been measured in large signal measurements.

Here the measurement calibration has been done at a fundamental frequency of $5 \mathrm{GHz}$. The measurement method consists in performing periodic $\mathrm{CW}$ time-domain large-signal load-pull RF measurements using Large-Signal Network Analyzer (LSNA). The LSNA can measure the harmonics of the high voltages and high currents at the DUT planes from $1 \mathrm{GHz}$ up to $18 \mathrm{GHz}$ with a dedicated test bench. Thanks to on-wafer absolute and relative calibrations with absolute phase reference of LSNA, the time-domain RF voltages and currents (at fundamental and harmonic frequency [fo, $2 \mathrm{fO}$ and $3 \mathrm{fo}]$ ) and periodic $\mathrm{CW}$ power performance are obtained at the DUT planes. The choice of this frequency of $5 \mathrm{GHz}$ was made for the reason that the equivalent capacitance versus frequency (as shown in Figure III.32) do not change up to around $8 \mathrm{GHz}$, and because one of these studied varactor will be used for frequency reconfigurable antenna around $7 \mathrm{GHz}$. For this, large signal analysis will be conducted around $5 \mathrm{GHz}$ and we assume that this analysis is valid for all frequencies below $8 \mathrm{GHz}$. In addition, the RF power was swept from $-15 \mathrm{dBm}$ to $18 \mathrm{dBm}$ (maximum output power from the source) while the $\mathrm{V}_{\mathrm{G}}$ was also swept from - $3 \mathrm{~V}$ to 6 with a step of $-0.2 \mathrm{~V}$.

The aim of this section is to propose an accurate analytical large signal model for GaN varactor diodes that could be used as general model, which offers the 
ability to describe the behavior of the device at high power levels and for various bias points. The large signal analysis of the devices, which includes the impact of RF power on the $\mathrm{C}_{\mathrm{EQ}}$ and $\mathrm{R}_{\mathrm{EQ}}$ values is established and explained. In addition, the nonlinear model behavior of $\mathrm{C}_{\mathrm{EQ}}-\mathrm{V}_{\mathrm{G}}$ characteristics is described by a simple analytical expression based on empirical coefficients which are derived from the large signal measurements. This developed analytical equation is valid for a linear size-scaling and is introduced for the voltage and RF power dependency of $\mathrm{C}_{\mathrm{EQ}}$.

\subsubsection{Analysis of Equivalent Capacitance}

In order to describe the nonlinear behavior of $\mathrm{C}_{\mathrm{Eq}}$ impacted by the injected $\mathrm{RF}$ power, an analytical equation based on empirical coefficients is derived from the measurements. This developed equation (see Equation (8)) is based on the one (Equation (3)) proposed before to describe $\mathrm{C}_{\mathrm{EQ}}-\mathrm{V}_{\mathrm{G}}$ curve for small signal which was only $\mathrm{V}_{\mathrm{G}}$ dependency. However, the influence of $\mathrm{RF}$ power has been taken into account in Equation (8) to approximately describe $\mathrm{C}_{\mathrm{Eq}}$ versus $\mathrm{V}_{\mathrm{G}}$ for various power levels. It is seen that Equation (8) accurately describes the dependency of junction capacitance upon applied reverse bias as well as upon RF power for all characterized devices. The $\mathrm{C}_{\mathrm{Eq}}-\mathrm{V}_{\mathrm{G}}$ curve can be expressed by the following analytic equation:

Equation (8)

$$
C e q=\left(\frac{B}{C} A\right)\left[\left(1+\frac{1}{C}\right)\left(e^{\tanh [(V g+F)(G-H P i n)+I]}\right)+k\right]
$$

Where $\mathrm{A}$ is the finger area. The parameters $\mathrm{B}, \mathrm{C}, \mathrm{F}, \mathrm{G}, \mathrm{H}, \mathrm{I}$ and $\mathrm{K}$ were adjusted to properly fit the $\mathrm{C}_{\mathrm{Eq}}$ equation to the measured characteristics of an arbitrary varactor size. According to Matlab simulations, the values of these empirical coefficients can be fixed for all devices, respectively, to $67,44.4,4.1,1.5$, 0.0565, 0.4 and 2.8. Equation (8) is dependent only on three main parameters, which are: finger geometry (number, width and length of fingers), bias voltage $\left(V_{G}\right)$ and $R F$ power $\left(\mathrm{P}_{\text {in }}\right)$. Due to their small size and to avoid breaking down the devices, we did 
not use any driver power amplifier to apply larger RF signals. It was noticed that above $5 \mathrm{dBm}$, the value of $\mathrm{C}_{\mathrm{Eq}}$ starts being affected by the power level.

Figure III.37, Figure III.38 and Figure III.39 show the measured and the modeled equivalent capacitance values versus $V_{G}$ for various $R F$ power levels $\left(P_{\text {in }}\right)$, respectively, for W50L0.5, W100L0.5 and W200L0.5. It can be seen that all $\mathrm{C}_{\mathrm{Eq}}-\mathrm{V}_{\mathrm{G}}$ curves exhibit the same general behavior. Under small power levels up to $5 \mathrm{dBm}$, this behavior can be separated into three regions which are range (1) for $V_{G}$ above $3.4 \mathrm{~V}$, range (2) for $-4.5 \mathrm{~V} \leq \mathrm{V}_{\mathrm{G}} \leq-3.4 \mathrm{~V}$ and range (3) for $\mathrm{V}_{\mathrm{G}}<-4.5 \mathrm{~V}$. However, more the power level increases more range (2) is extended. For range (1) and range (3) corresponding to the device's maximum $\left(\mathrm{C}_{\mathrm{MAX}}\right)$ and minimum $\left(\mathrm{C}_{\mathrm{MIN}}\right)$ values, the capacitance values do not change significantly versus $V_{G}$. In range (2), the capacitance values change appreciably and rapidly over a small DC bias range from $-4.5 \mathrm{~V}$ to $-3.4 \mathrm{~V}$. This bias range extends as well as the power is increased. However, the $\mathrm{C}_{\text {MAX }}$ and the $\mathrm{C}_{\text {MIN }}$ values, respectively, decrease and increase as well while the input power increases. This means that the $\mathrm{C}_{\mathrm{MAX}} / \mathrm{C}_{\mathrm{MIN}}$ ratio drops significantly, resulting in device saturation and non-linearity at high power levels.
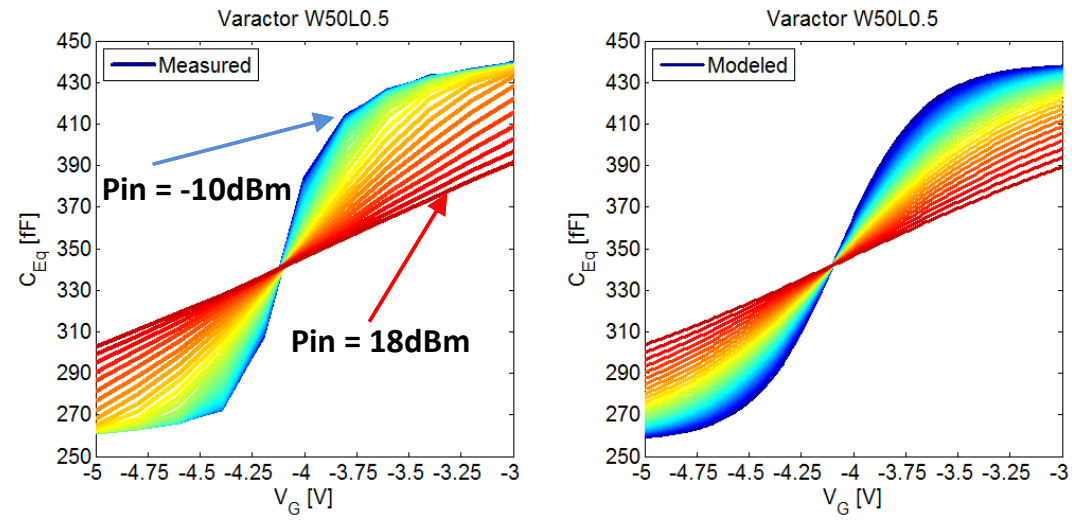

Figure III.37: Extracted (left) and Simulated (right) $C_{E q}$ versus $V_{G}$ for various power levels $[-10,18] \mathrm{dBm}$ for device W50L0.5 

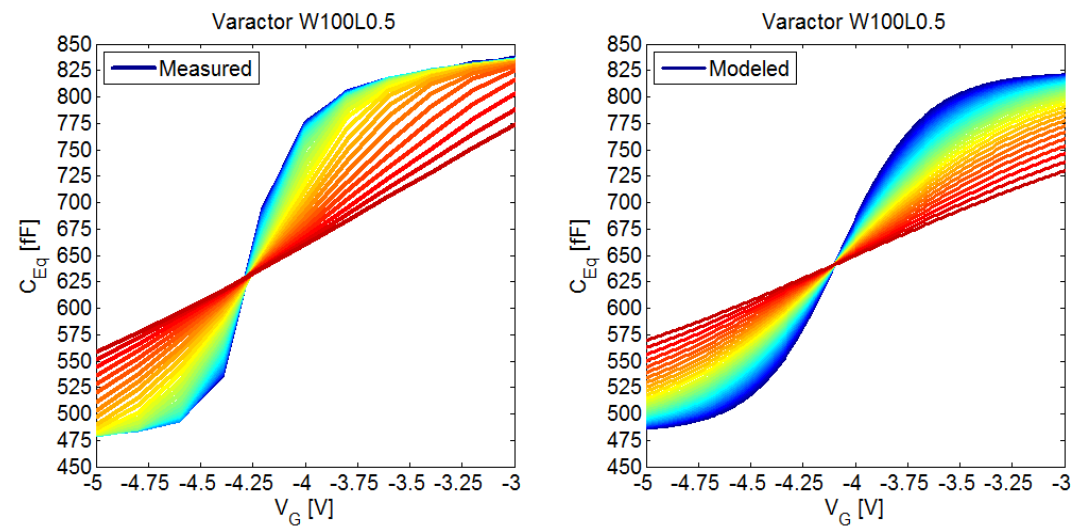

Figure III.38: Extracted (left) and Simulated (right) $C_{E q}$ versus $V_{G}$ for various power levels $[-10,18] \mathrm{dBm}$ for device W100L0.5
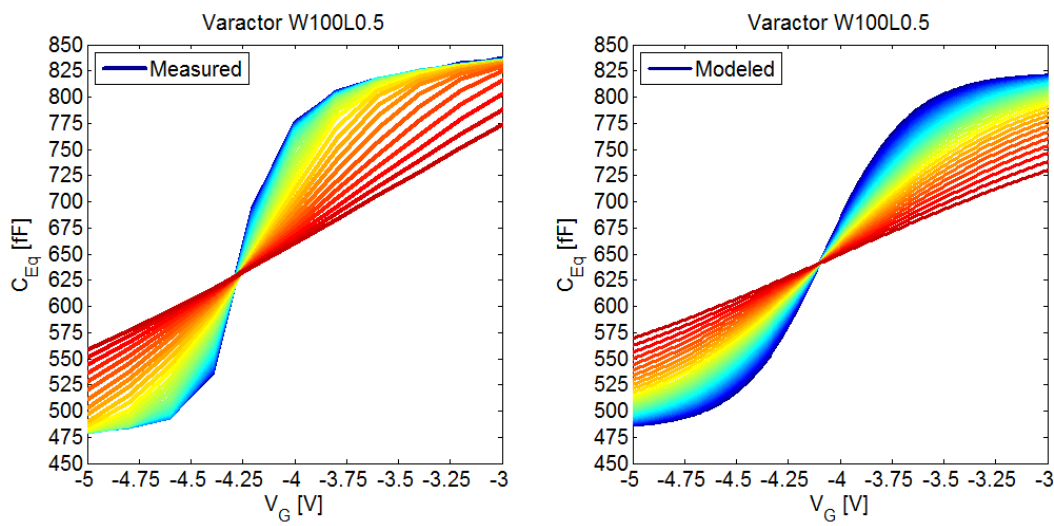

Figure III.39: Extracted (left) and Simulated (right) $C_{E q}$ versus $V_{G}$ for various power levels $[-10,18] \mathrm{dBm}$ for device W200LO.5

\subsubsection{Analysis of Equivalent Series Resistance}

$R_{\mathrm{Eq}}$ versus $\mathrm{V}_{\mathrm{G}}$ for various power levels are extracted and are depicted in Figure III.40. As can be seen from the $\mathrm{R}_{\mathrm{Eq}}-\mathrm{V}_{\mathrm{G}}$ characteristics, the extracted value of $\mathrm{R}_{\mathrm{Eq}}$ varies from $3 \Omega$ to $6 \Omega$ for W50L0.5, from $2.7 \Omega$ to $4.4 \Omega$ for W100L0.5 and from $3.4 \Omega$ to $4.4 \Omega$ for W200L0.5. So a mean value can be fixed to describe the behavior of the series resistor at any RF power level from $-10 \mathrm{dBm}$ to $18 \mathrm{dBm}$, as well as at any bias voltage $V_{\mathrm{G}}$. These values of $\mathrm{R}_{\mathrm{Eq}}$ are significantly smaller than those reported before for the GaN500 v1 devices. Due to these series resistor, a part of the RF available power $\left(\mathrm{P}_{\mathrm{av}}\right)$ is consumed by the devices. For example, for the W50L0.5 
varactor (the one has highest $R_{E q}$ ), Figure III.42 clearly illustrates this power consumption $\left(\mathrm{P}_{\text {con }}\right)$ which follows the variation of the $\mathrm{R}_{\mathrm{Eq}}$ and which slightly impacted by voltage $V_{G}$ and the RF power. Figure III.41 (left) meanwhile depicts the power at the input of the device $\left(\mathrm{P}_{\mathrm{av}}\right)$ after removing losses through the $\mathrm{RF}$ cable (between source and device under test) and Figure III.41 (right) illustrates the reflected power $\left(\mathrm{P}_{\mathrm{ref}}\right)$ by the varactor. $\mathrm{P}_{\text {src }}$ represents the power delivered by the source.
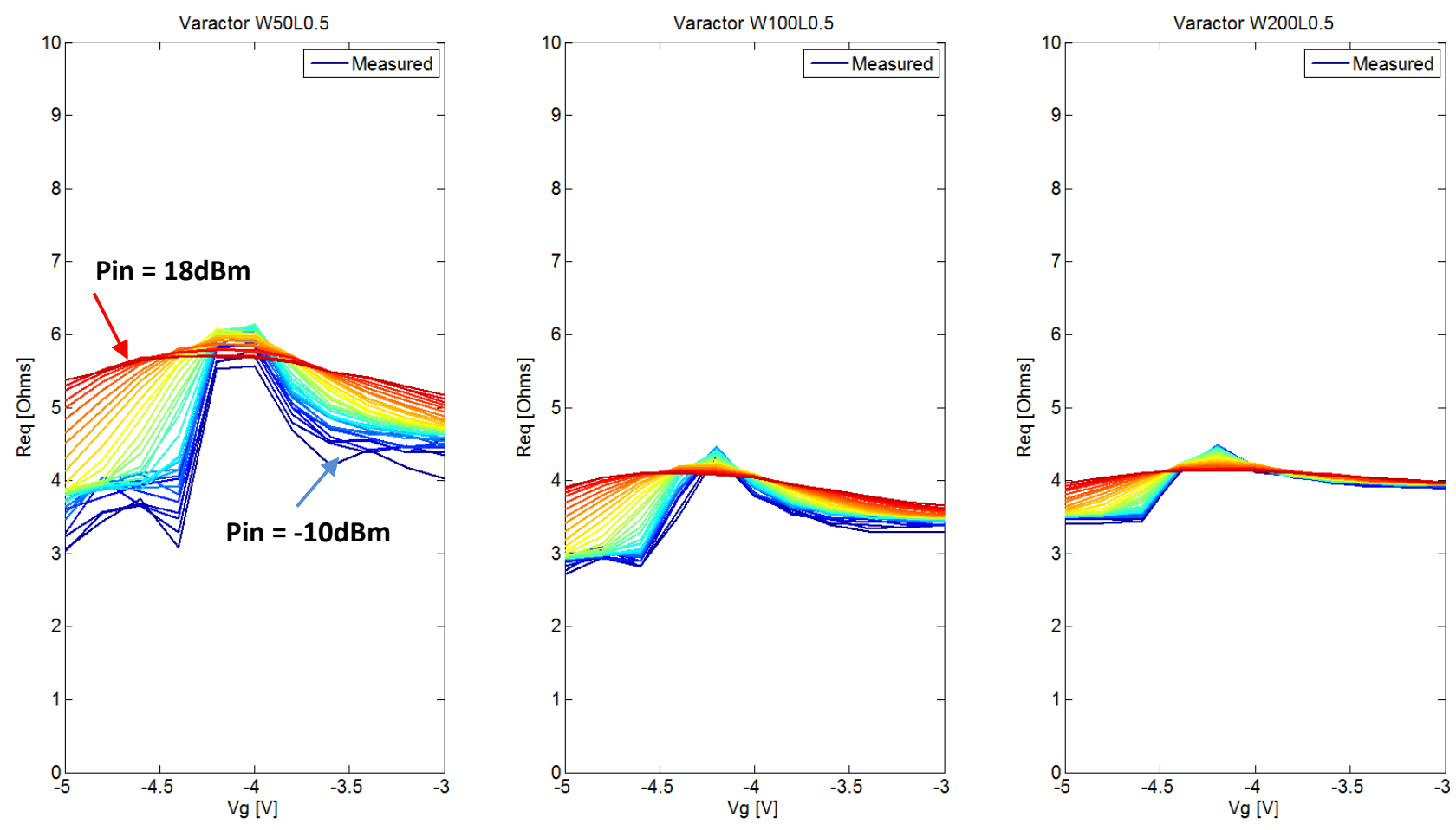

Figure III.40: Extracted $R_{E q}$ versus $V_{G}$ for various power levels [-10, 18] dBm for device W50L0.5 (left), for W100L0.5 (middle) and for W200L0.5 (right) 

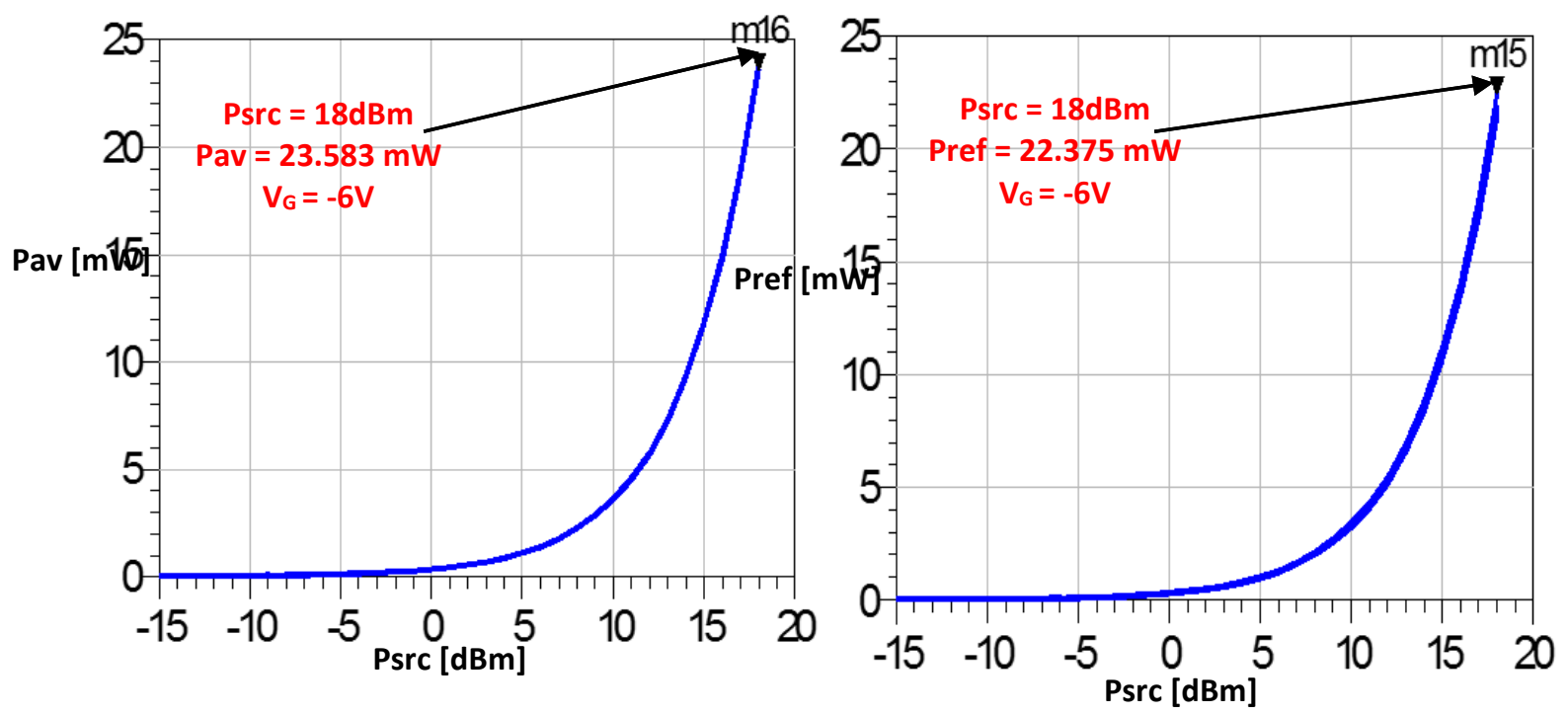

Figure III.41: Extracted Available (left) and reflected (right) RF Power versus

Power from Source for various DC Points for W50L0.5

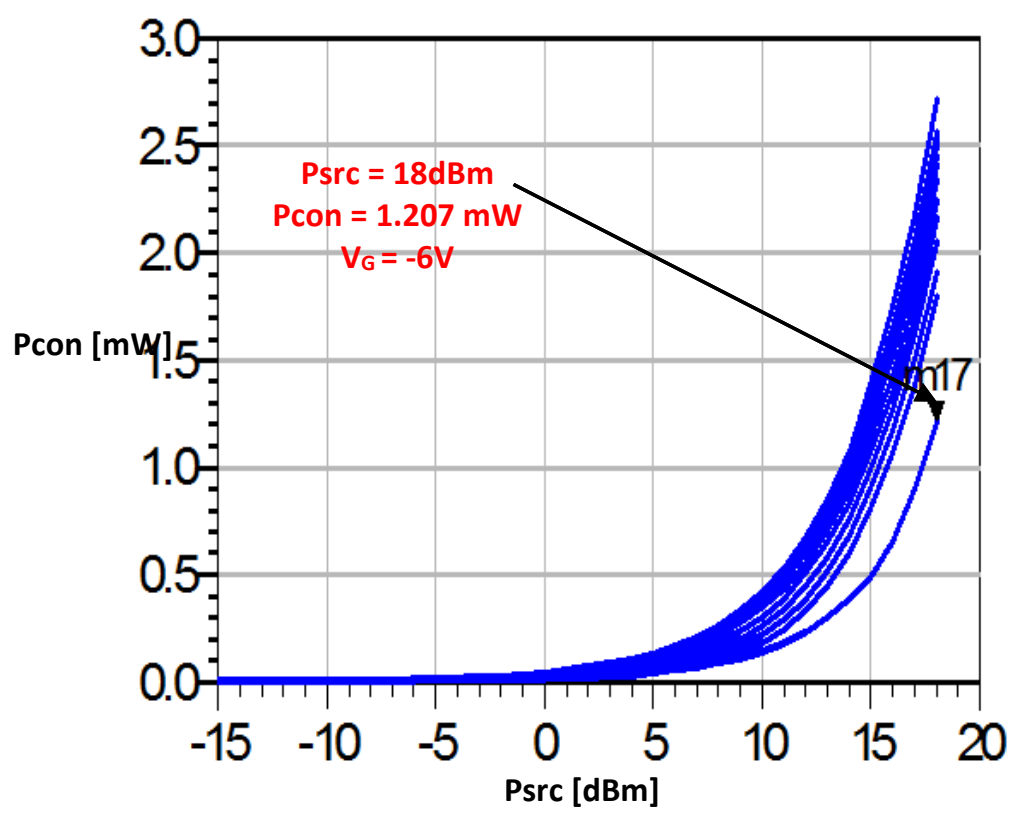

Figure III.42: Extracted Delivered RF Power versus Power from Source for various DC Points for W50L0.5 


\section{Summary}

The varactor devices structure studied here is based on NRC GaN processes. Three GaN HEMTs process, such as, GaN150v0 with a gate length of 0.15 um, GaN500v1 with gate length of $0.5 \mathrm{um}$ and GaN500v2 which is the developed version of GaN500v1, have been used for manufacturing the varactors diodes.

Small signal analysis has been done for all fabricated devices. Equivalent capacitance and series resistor behaviors have been detailed, and it was revealed that all $\mathrm{C}_{\mathrm{Eq}}$ versus $\mathrm{V}_{\mathrm{G}}$ exhibit the same general behavior for all devices. In addition, the capacitance tuning range is limited, and the region of variable capacitance exists only over a small reverse voltage of $1 \mathrm{~V}$. Furthermore, the capacitance value depends on the finger area. This means that larger is the device, higher are the capacitance values. Moreover, it was observed that the extracted capacitance values from varactors with GaN500v2 are at least twice larger than those extracted with the same device size fabricated with GaN500v1 process. This is due to the additional field plate layer in GaN500v2 process. It was, also, observed that the devices manufactured with GaN500v2 and GaN150v0 have lower series resistor value than those value reported with GaN500v1 devices. Two analytic equations describing the behavior of equivalent capacitance and series resistor versus bias voltage were proposed. These equations are only bias voltage and device geometry dependency.

Large signal analyses have been done only for devices manufactured with GaN500v2 process. The influence of RF power has been taken into account and an analytic expression was proposed to approximately describe equivalent capacitance versus bias voltage for various power levels. This equation accurately describes the dependency of junction capacitance upon applied reverse bias as well as upon RF power for all characterized devices.

This modeling work has led to one published journal paper [149] and one other submitted to IEEE Transactions on Device and Materials Reliability in August 
2016 [151]. A conference paper has been also presented in European Microwave Conferences in Paris in 2015 [150].

\section{GaN-based Switch Devices Modeling}

\section{Review of GaN HEMT for Power Switching Devices Applications}

As previously mentioned in the introduction section, GaN-based switch components is a part of the proposed thesis research. The main aim of this part is to conduct a complete modeling GaN-based switch study using a GaN HEMT process from NRC. Our investigation was planned to consider both small and large signal analysis. Here in this section, different sizes single standalone switches and switch structures such as single pole single throw (SPST) and single pole double throw (SPDT) were fabricated using NRC GaN500v2 and will be presented. Due to our incapability to have large signal measurement facilities, only small signal measurement will be presented and discussed. An accurate large signal model requires extensive laboratory equipment to extract all the effects related to high power GaN devices, such as thermal and trapping effects typically present in GaN devices. Carleton University is not as well equipped with these laboratory facilities as are the NRC or IETR.

The fabricated switch circuits will be integrated with antenna system to realize and to demonstrate the ability of using GaN devices for a steering beam antenna array that will be presented later in chapter IV.

As a promising high power handling alternative, GaN technology is expected to be a good candidate for the realization of high power HEMT-based switches [69]-[70]. The HEMTs suitability for switch usage stems from the fact that the RF path between source and drain is considered as a voltage-controlled resistor [71]. The DC bias applied to the gate controls the two states of switching "ON and 
OFF states", with no DC bias applied to source or drain. In addition to this controlledresistance there is also a capacitance associated with the HEMT structure. In other words, the equivalent circuit of a HEMT-based switch could be a simple variable resistor and capacitor in the path of the RF signal, as shown in Figure III.43. However, in practice a more complex model is used to model the GaN HEMTs-based switch. Subsection d explains in detail the modeling of the GaN switch.

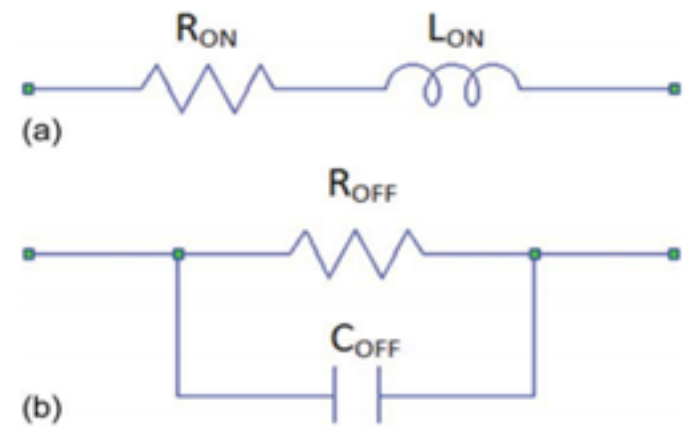

Figure III.43: Simple Model for a HEMT in the switch configuration (a) ON-state and (b) OFF-state mode

Over the last fifteen years, GaN HEMT-based switch circuits started appearing among the other semiconductor technologies, due to their improved material quality which gives GaN power switching devices an advantage for high power/frequency applications. The first preliminary investigation for RF switching devices using GaN has been suggested in 2001 [72]. Several reported works are based on simple standalone transistor or single pole double throw (SPDT) switch structures. They demonstrated an extremely linear power handling of $43 \mathrm{~W}$ (10 times higher than that of typical GaAs-based switch ICs) at $1 \mathrm{GHz}$ with excellent performance in terms of insertion loss (-0.26 dB) and isolation (-27 dB) [73]-[74]. Another AlGaN/GaN HEMT SPDT switch [75] has been realized and the measurements showed values of $-0.87,-0.96,-1 \mathrm{~dB}$ insertion loss and $-46,-42$ and $-41 \mathrm{~dB}$ isolation at $0.9,1.8$ and 2.1 $\mathrm{GHz}$ respectively. The switch also shows linear performance for power levels up to 1 Watt in the insertion mode and more than 2 Watts in the isolation mode. Due to its good characteristics, GaN technology could be extended to high frequency switch 
applications without the need for purely resonant operation. This leads to some advantages such as switch size and ease of design [76]. As a result, many SPDT switches have already been successfully demonstrated around X-band, Ku-band or higher frequency. One of these works is [77] where an AlGaN/GaN HEMT Ku-band SPDT switch has been reported with an insertion loss of $-1.4 \mathrm{~dB}$, isolation of $-35 \mathrm{~dB}$, and $36 \mathrm{dBm}$ output power at $18 \mathrm{GHz}$. Clearly, this work demonstrates the high power capabilities of GaN technology at high frequency for switch device.

Improving the isolation of $\mathrm{GaN}$ switch circuits over large frequency bands was a reason leading to the use of resonance inductors in parallel with the HEMTs. Based on this technique, [78] presents an X-Band GaN SPDT MMIC switch with over 25 Watt linear power handling. The achieved isolation, for this work, is around -35 $\mathrm{dB}$ in $\mathrm{X}$-band, while the insertion loss was high and was about - $3.5 \mathrm{~dB}$. This insertion loss is high due to the on-resistor value of the device. Continuing with the same concept, a PhD student, Tyler Neil Ross, from the Department of Electronics (DOE) at Carleton University, developed high-power broadband GaN HEMT SPST/SPDT switches based on resonance inductors and shunt-stacked transistors [79]. For the SPST an insertion loss less than $-3 \mathrm{~dB}$ and isolation better than $-13 \mathrm{~dB}$ from $\mathrm{DC}$ to $20 \mathrm{GHz}$ were achieved, while for SPDT an insertion loss better than $-2 \mathrm{~dB}$, an isolation higher than $-20 \mathrm{~dB}$ and a power handling at $1 \mathrm{~dB}$ compression $(\mathrm{P} 1 \mathrm{~dB})$ greater than $10 \mathrm{~W}$ were reported. Going higher in frequency, in [80] a Ka-Band high power GaN SPDT switch MMIC was demonstrated with unprecedented $49 \mathrm{dBm}$ at P1dB. Measured insertion loss is better than $-1.3 \mathrm{~dB}$ over $27-31 \mathrm{GHz}$, and the minimum insertion loss of - $0.8 \mathrm{~dB}$ has been achieved at higher frequencies. Measured isolation is greater than $-25 \mathrm{~dB}$ over $27-31 \mathrm{GHz}$, with the highest isolation of $-30 \mathrm{~dB}$ at higher frequencies.

Clearly, this literature review shows that GaN-based switches can achieve impressive high power handling capability beyond that of other wide band 
semiconductor technologies. In addition, excellent performances in terms of isolation and insertion loss can be achieved.

\section{NRC GaN Switch Modeling}

The AlGaN/GaN HEMTs switches investigated in this thesis work were fabricated by the National Research Council of Canada (NRC). In this process, there is considerable doubt that the provided models are accurate for switching applications because only transistors in common source configuration for power amplifier purposes are provided. In this section, a brief review of $\mathrm{GaN}$ modeling will be discussed. Then, a small-signal transistor model useful for analyzing and designing GaN switching circuits using this foundry will be presented.

\subsection{Proposed GaN Single Switch Model}

The modeling part is crucial in HEMT circuit designs. A good model certainly relies on a circuit simulation tool that provides the capability to determine circuit performance with high accuracy against prototype measurements. Also, it allows to carry out a good verification of all the different test structures, leading to prediction of the transistor's overall physical phenomena. However, to cover all the major effects influencing directly the HEMT's characteristics and performance, it is necessary to present a good model that is as general as possible.

For GaN HEMT technology, there has been interest in modeling transistors as basic building blocks. Actually, most of these efforts have focused on empirical [81]-[82] and semi-physical formulations. GaAs and GaN technology are commonly referred to as wide bandgap compound semiconductors, which lead to the fact that the extraction of GaN HEMT parameters mostly follows the same procedures as those for GaAs HEMTs, with a few exceptions. GaN HEMTs have much higher conduction bands than the corresponding GaAs HEMTs. As a result, GaN 
models are based on GaAs models, and are then extended or modified to better fit into the form of GaN device's properties.

Various types of models have been employed for HEMTs, most of them are semi-empirical. Therefore, models are today based only on formulas which, also, seem to work for HEMT devices. For example, the hyperbolic tangent function is employed to approximate drain current. For GaN HEMTs, the most important effects which should be considered in a nonlinear modeling are:

> Self-heating: Transistor's ability to predict the dynamic changes of its temperature during operation due to power dissipation [83].

> Nonlinear charge modeling, Qgs, Qds and Qgd, has been shown to be critical to accurately simulating bias dependence on all three terminal voltages (Gate, Drain and Source) [83].

$>$ Surface traps effects where gate-lag effects and drain-lag effects are included [84]-[85].

An accurate model is imperatively related to the measure of all reported effects. A mismeasurement or a lack of one affects the results by improperly extracting the device's parameters [86]-[87]. An overly simple model can have significant effect at high power, leading to fail the device's performance. They are three main types of GaN HEMTs device's equivalent models, which are Chalmers model [81]-[88], EEHMEMT model from Agilent and Curtice model [83]-[89]. Chalmers model is mostly used where many properties of transistor are included [84].

As mentioned, the devices presented in this study were manufactured by NRC in Ottawa. To accurately capture the performances of switch circuits, a complex model is needed to properly model the GaN HEMT-based switch. NRC's GaN500 process foundry does not provide a transistor model suitable for switch designs. As a matter of fact, a challenging task resided in developing an accurate GaN HEMT model for NRC's GaN500v2 process as a switch that it will be used for our purpose simulations. 
A linear model, as shown in Figure III.45, has already been presented by Tyler Ross in [[90] using NRC's GaN500v1 process. This model is inspired from [91] where a linear model for GaAs HEMT is reported (see Figure III.44). Based on this available model several SPST and SPDT switch structures and standalone transistors (common gate) with different sizes were submitted to NRC for fabrication in GaN500v2. The idea, for this thesis GaN switches section, was to redo the small signal modeling by using GaN500v2 process and then for the first time the large signal modeling using this foundry. Unfortunately, as already mentioned, due to our incapability to have large signal measurement facilities, we are not able to provide measured results or complete the characterization for large signal study as was planned.

Using GaN500v1, some performances advantages have been achieved in terms of high breakdown voltage, low on-resistor and low leakage current at the gate [[30]. This leads us to assume that the switch designs using GaN500v2 could have better performances compared to the ones fabricated using GaN500v1.

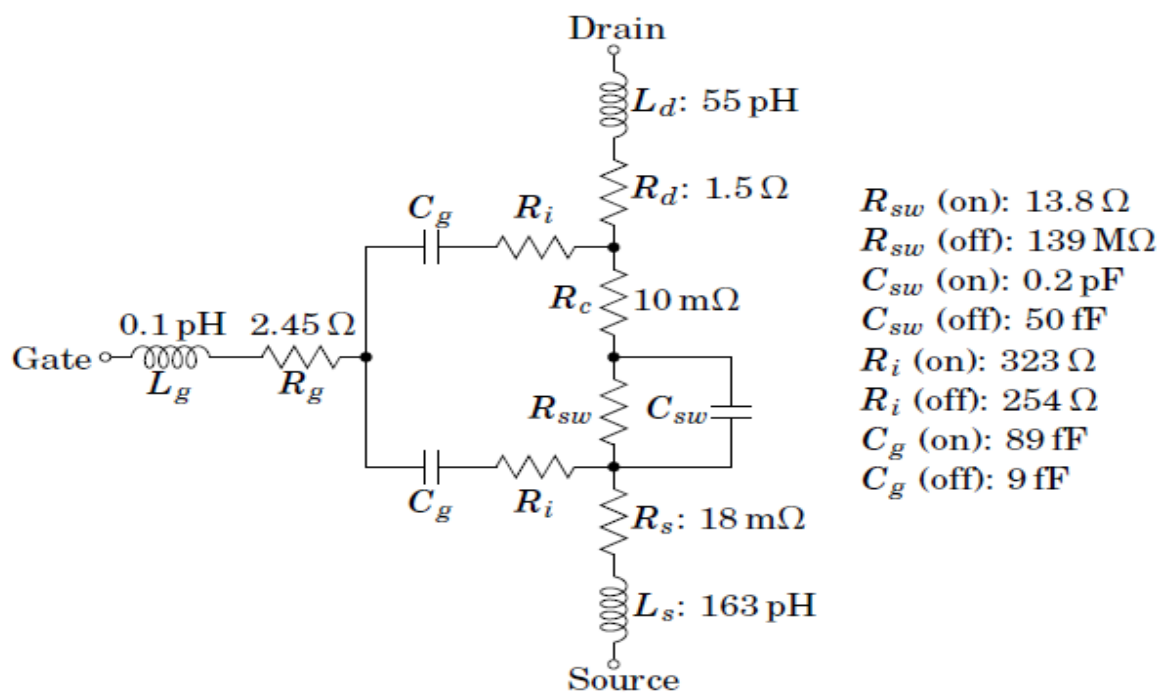

Figure III.44: Linear model used to model the switching GaN HEMT transistors $(4 x 40)[[90]$ 


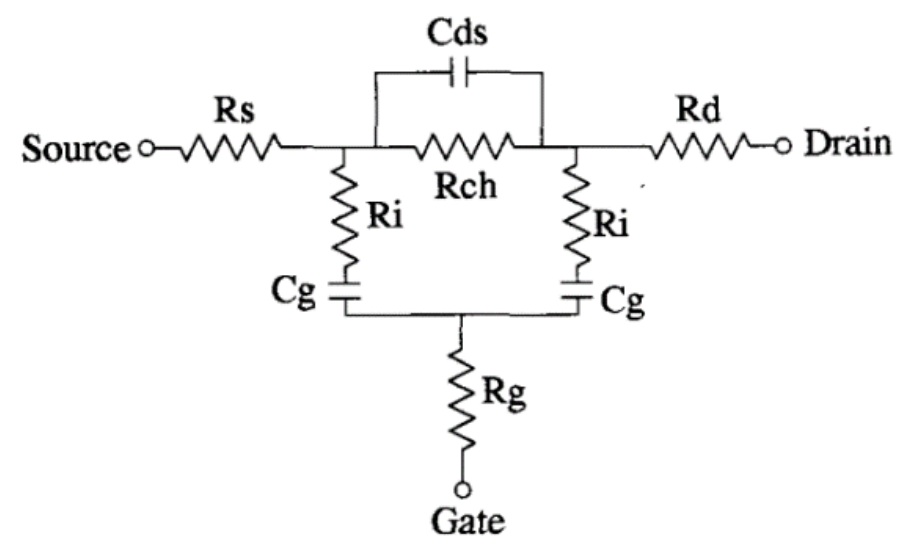

\begin{tabular}{ccc} 
Parameter & ON State & OFF State \\
\hline $\operatorname{Rs}(\Omega)$ & 0.4675 & 0.4675 \\
$\operatorname{Rd}(\Omega)$ & 0.4691 & 0.4691 \\
$\operatorname{Rg}(\Omega)$ & 33.97 & 33.97 \\
$\operatorname{Ri}(\Omega)$ & 11.85 & 82.95 \\
$\operatorname{Rch}(\Omega)$ & 1.58 & $39,500.00$ \\
$\operatorname{Cg}(\mathrm{pF})$ & 0.6962 & 0.06329 \\
$\operatorname{Cds}(\mathrm{pF})$ & 0.2405 & 0.16835 \\
Switch FET Model Parameters Values Normalized \\
to $1.0 \mathrm{~mm}$.
\end{tabular}

Figure III.45: Equivalent Linear Circuit Model of GaAs FET Switch [91]

The reported currently available simple linear model can be useful to estimate the power-handling capability of a switch circuit. The model presented can be easily integrated into the full circuit simulation, and the voltages across the transistor's terminals and the current through the device can be determined using a linear ac analysis available in virtually all simulators. As long as the input RF power does not induce a voltage swing across transistor's terminals that reaches the transistor's pinch-off voltage or breakdown voltage, the switch-based circuit should function largely as predicted from a linear S-parameter analysis. This simulation does not lend itself well to a quantitative prediction of the $1 \mathrm{~dB}$ compression point or input-referred third-order intercept point (IIP3). However, it does provide a designer with some useful information on the power-handling capability of the circuit, which can be refined once a large-signal model can be extracted, or when measurements are performed. 


\section{GaN500v2 Small Signal Switches Measurements}

\subsection{Different Sizes Standalone Signal Switch Devices}

Here the conventional measurements setup of a transistor in common gate configuration is described. The simplified schematic presented in Figure III.46 (left) is used as a test bench to characterize the standalone switches while the Figure III.46 (right) illustrates the manufactured design for one switch device. As the ability to control the current through the switch should be independent of the current flowing, then it is recommended to put a strong gate resistance, $R_{\text {bias }}$, for isolation between the control and RF signals. The value of this resistor was chose to be $10 \mathrm{~K} \Omega$. The onwafer RF measurements were performed with an Agilent VNA Network Analyzer over a frequency range of $0.1-10 \mathrm{GHz}$. The control voltage at the gate, $\mathrm{V}_{\mathrm{G}}$, was switched between $0 \mathrm{~V}$ and $-5 \mathrm{~V}$ to control the switch, respectively, for $\mathrm{ON}$ and $\mathrm{OFF}$ states.
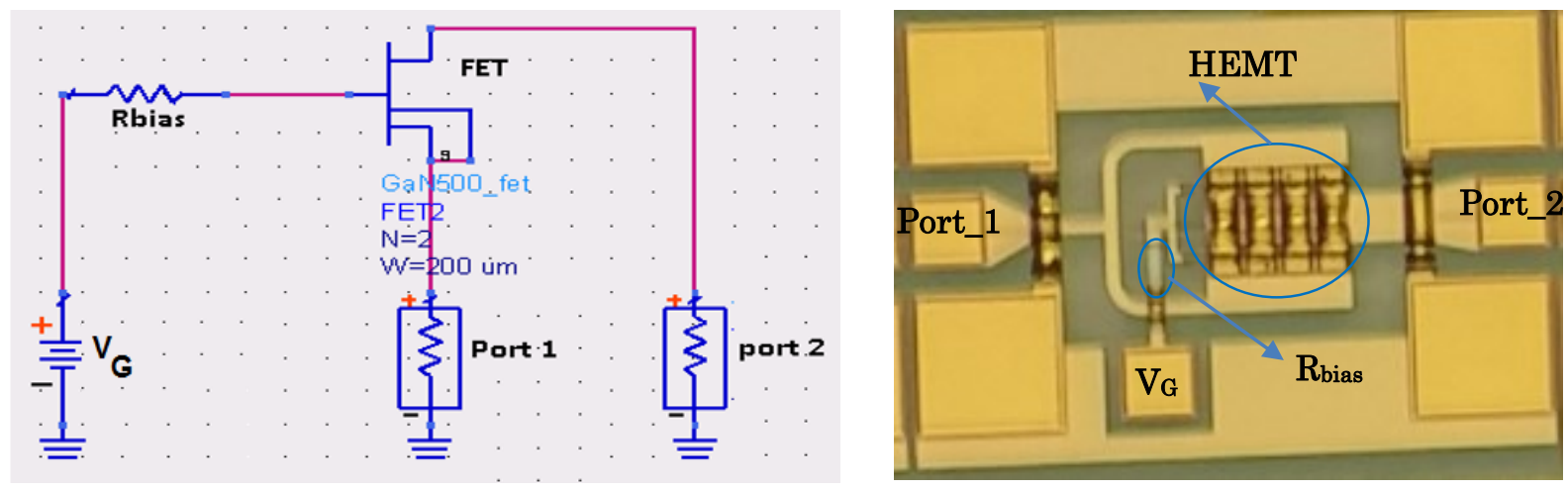

Figure III.46: Schematic (left) of Common Gate Configuration standalone switch 2x200_GaN500v2 and Device in Microwave probes measurement setup (right) to Evaluate RF behavior

Figure III.47, Figure III.48, Figure III.49 and Figure III.50 show, respectively, the insertion loss (ON state), the isolation (OFF state), and the return loss at $\mathrm{ON}$ state and at $\mathrm{OFF}$ state for the different sizes realized standalone single 
switches. The measurements were limited at $10 \mathrm{GHz}$ for two reasons. First, it was observed that the performances of the measured devices decrease at high frequency in terms of insertion loss and isolation. Second, the realized final structure is planning to be used for beam switching antenna at $2.45 \mathrm{GHz}$.

As can be seen, the isolation is good at low frequencies but quickly drops with increasing frequency. So, it can be said that the larger the device used, the lower the insertion loss is achieved but the poorer the isolation. These obtained results are in good agreement with the reported small signal analysis in [[90] using GaN500v1 from NRC.

A trade-off between insertion loss and isolation should be made to choose the single transistors that will be used for designing switch structure such as SPST and SPDT. Switch structures such as SPST and SPDT are very popular in semiconductor switches applications, they are used for improving the performances (insertion loss and isolation) over large frequency bands. Based on the investigated standalone switches, SPST and SPDT structures will be designed and measured result will be presented and discussed.

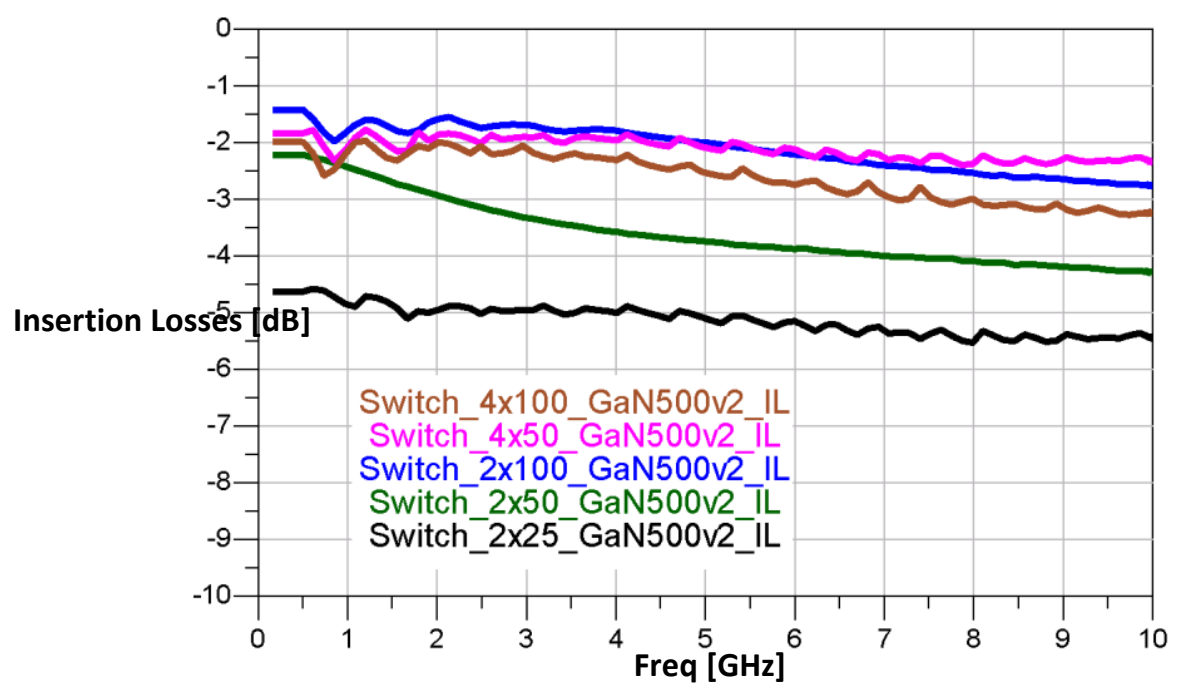

Figure III.47: Measured insertion loss of different transistor size in common gate, biased with $V_{G}=O V$ (ON state) 


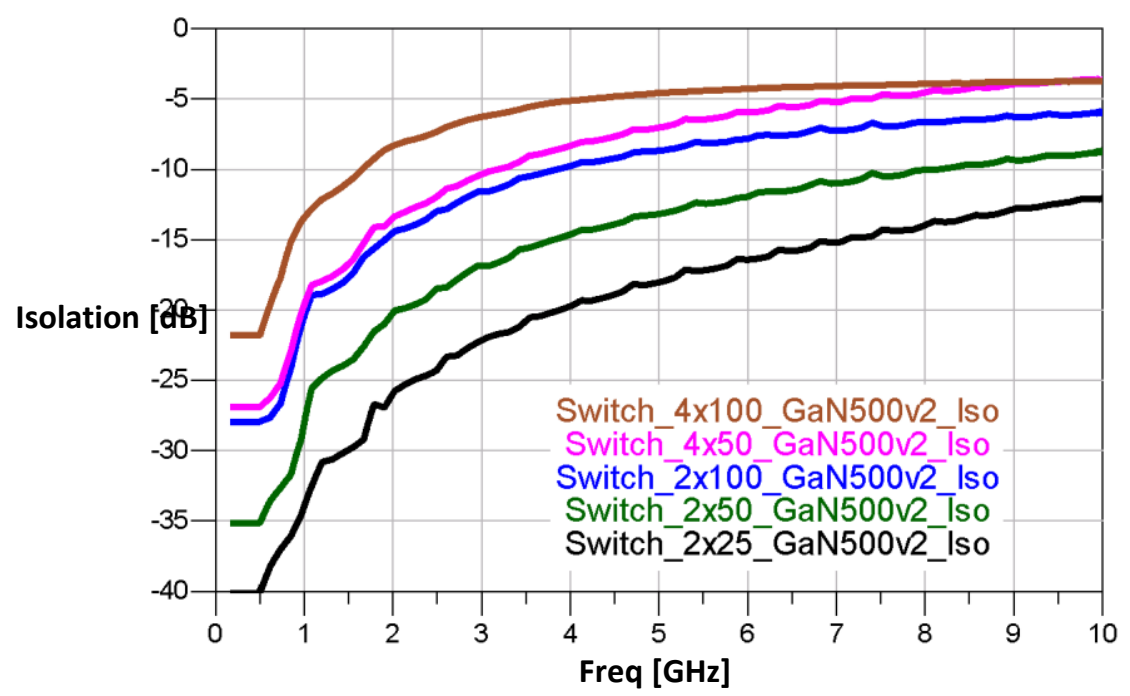

Figure III.48: Measured Isolation of different transistor size in common gate, biased with $V_{G}=-5 V(O F F$ state $)$

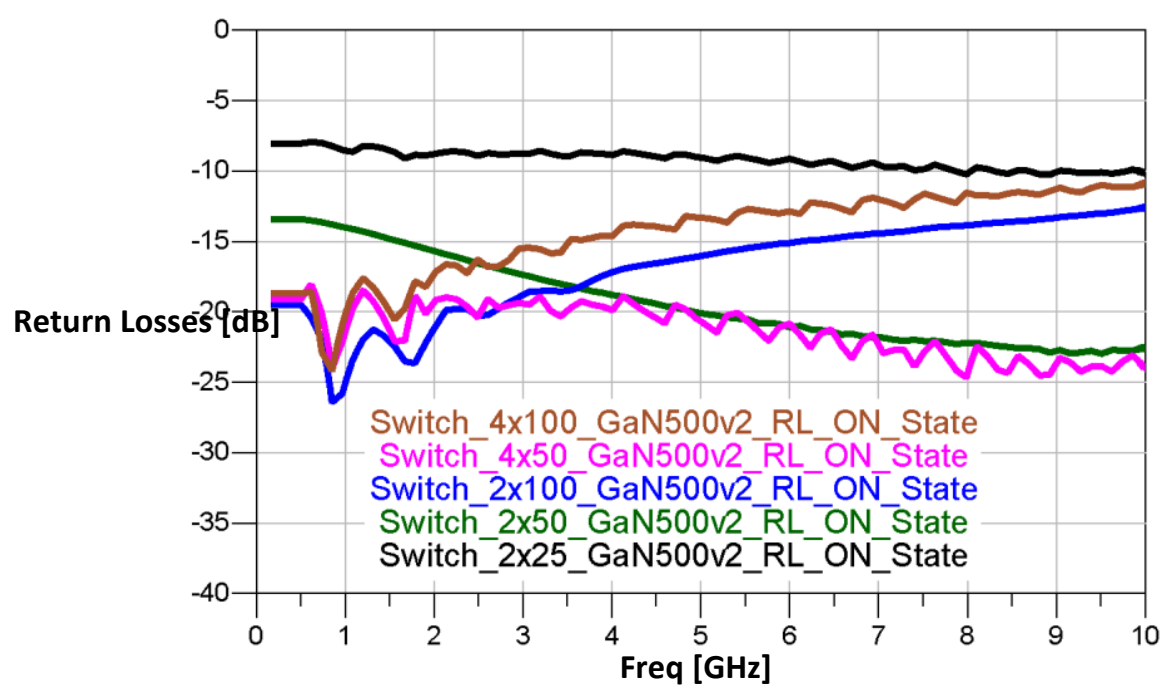

Figure III.49: Measured Return Loss (S11) of different transistor size in common gate, biased with $V_{G}=O V$ (ON state) 


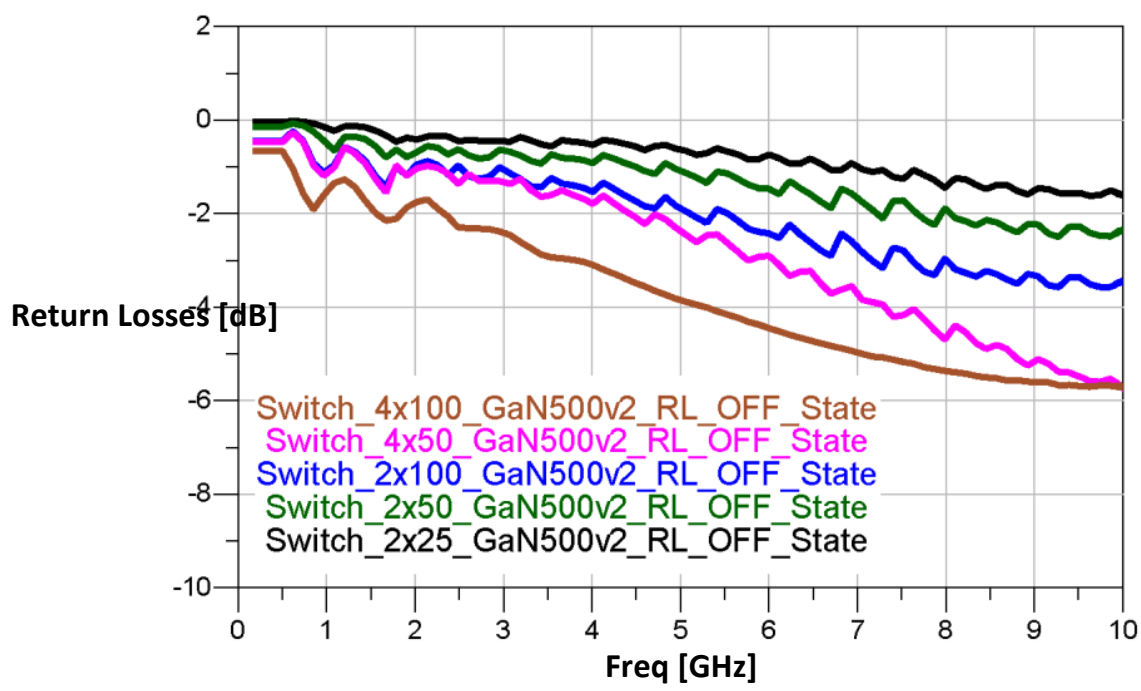

Figure III.50: Measured Return Loss (S11) of different transistor size in common gate, biased with $V_{G}=-5 V(O F F$ state $)$

Based on these small signal measurements, a model is introduced and detailed in Table III.7 and Figure III.51. It was observed that this linear model is the same to the one proposed in [[90] by Tyler Ross using GaN500v1 from NRC foundry. A comparison of the S-parameters of both the ON and OFF states is shown in Figure III.52 and Figure III.53, respectively. As shown, the fit between model and measurements is very good. In Table III.7, parameters corresponding to a single switch (2x25) with total gate width of $50 \mu \mathrm{m}$ and leaner scaleable with gate width are summarized. Cg, Ri, Rsw and Csw vary with gate bias as indicated.

Table III. 7: Model Circuit Parameters Values

\begin{tabular}{|c|c|c|}
\hline & ON State (OV) & OFF State (-5V) \\
\hline Gate Inductor (Lg) & $\mathbf{0 . 1 4} \mathrm{pH}$ & $\mathbf{0 . 1 4} \mathrm{pH}$ \\
\hline Gate resistor (Rg) & $3.92 \Omega$ & $3.92 \Omega$ \\
\hline Drain Inductor (Ld) & $65 \mathrm{pH}$ & $65 \mathrm{pH}$ \\
\hline Drain Resistor (Rd) & $\mathbf{7 . 7 4} \Omega$ & $7.74 \Omega$ \\
\hline Source Inductor (Ls) & $112 \mathrm{pH}$ & $112 \mathrm{pH}$ \\
\hline Source Resistor (Rs) & $\mathbf{2 6} \Omega$ & $\mathbf{2 6 \Omega}$ \\
\hline Rc & $16.6 \mathrm{~m} \Omega$ & $15.6 \mathrm{~m} \Omega$ \\
\hline Cg & $49.25 \mathrm{fF}$ & $36 \mathrm{fF}$ \\
\hline Ri & $178.8 \Omega$ & $396 \Omega$ \\
\hline Switch Resistor (Rsw) & $\mathbf{2 6 . 4 8 \Omega}$ & $\mathbf{2 7 7 . 4} \mathrm{M} \Omega$ \\
\hline Swicth Capacitor (Csw) & $\mathbf{0 . 3 9} \mathrm{pF}$ & $\mathbf{7 4 . 2 5} \mathrm{fF}$ \\
\hline
\end{tabular}




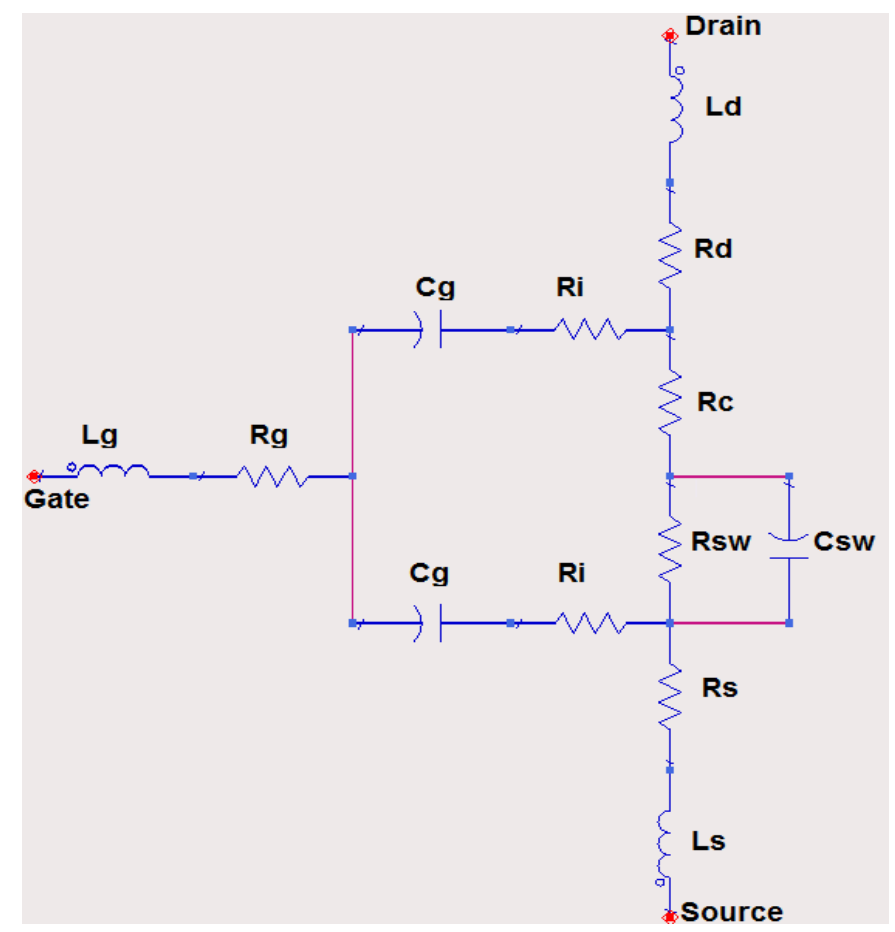

Figure III.51: Linear model used to model the switching GaN HEMT transistors.

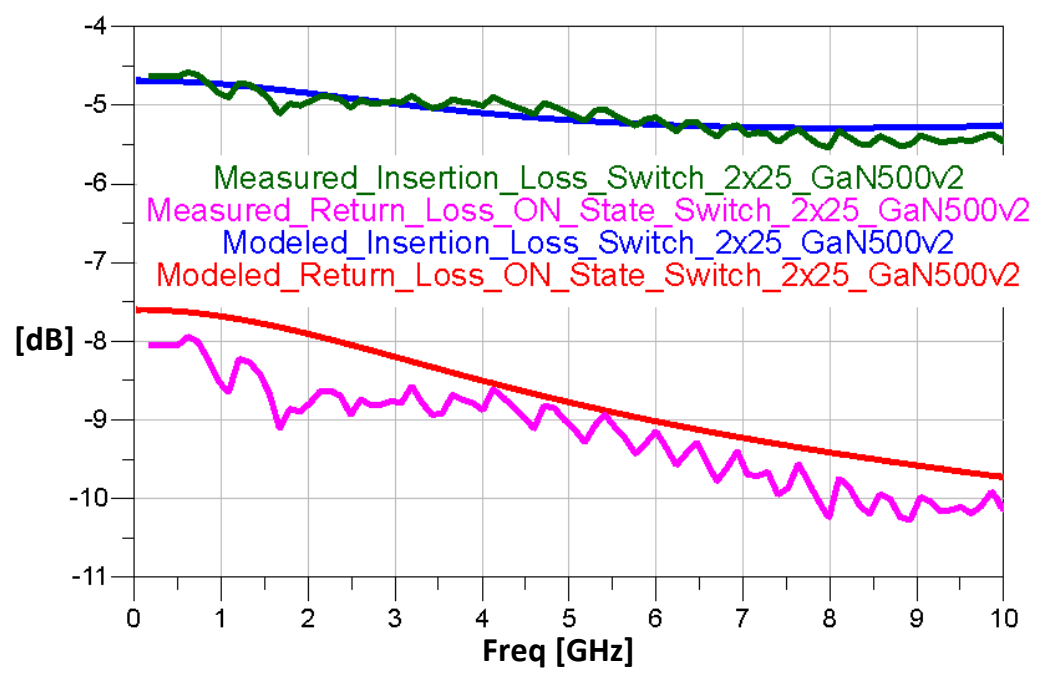

Figure III.52: Measured and Modeled Insertion Loss and Return Loss (S11) of switch $2 \times 25$ biased with $V_{G}=O V(O N$ state) 


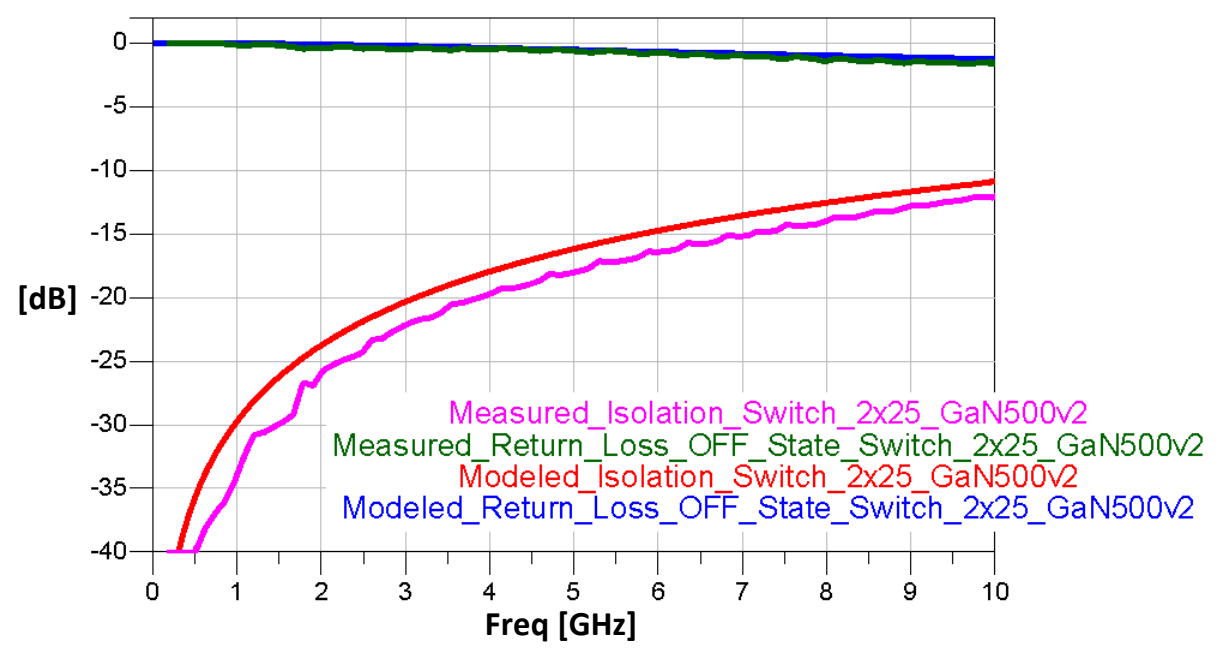

Figure III.53: Measured and Modeled Isolation and Return LosS (S11) of switch $2 \times 25$ biased with $V_{G}=-5 V(O F F$ state $)$

\subsection{SPST Switch Structure}

To improve the isolation and keep the insertion loss at its minimum, a SPST switch structure is proposed as in Figure III.54. A transistor with two fingers and a gate width of $100 \mu \mathrm{m}$ as used for the transmitted path to improve the insertion loss, while two transistors with 4 fingers and $50 \mu \mathrm{m}$ of gate width were used for the coupled path to improve the isolation. When the SPST switch operated in the ON state corresponding to $\mathrm{V}_{\mathrm{G} 1}=0 \mathrm{~V}$ and $\mathrm{V}_{\mathrm{G} 2}=-5 \mathrm{~V}$, FET1 is in the linear region as represented by a small on-resistor, and FET2 is in the pinch-off region, which can be presented as a small capacitor. Then the RF signal goes from the input port to the output port through the FET1 because input impedance is near $50 \Omega$. In the other hand, when the SPST switch is in the off-state, FET1 behaves as a small capacitor instead when a control voltage at $-5 \mathrm{~V}$ is applied for $\mathrm{V}_{\mathrm{G} 1}$, and FET2 can be treated as a small resistor by applying $0 \mathrm{~V}$ for $\mathrm{V}_{\mathrm{G} 2}$. Therefore, the input impedance is very high, and the input RF signals are completely reflected back to the input terminal. 

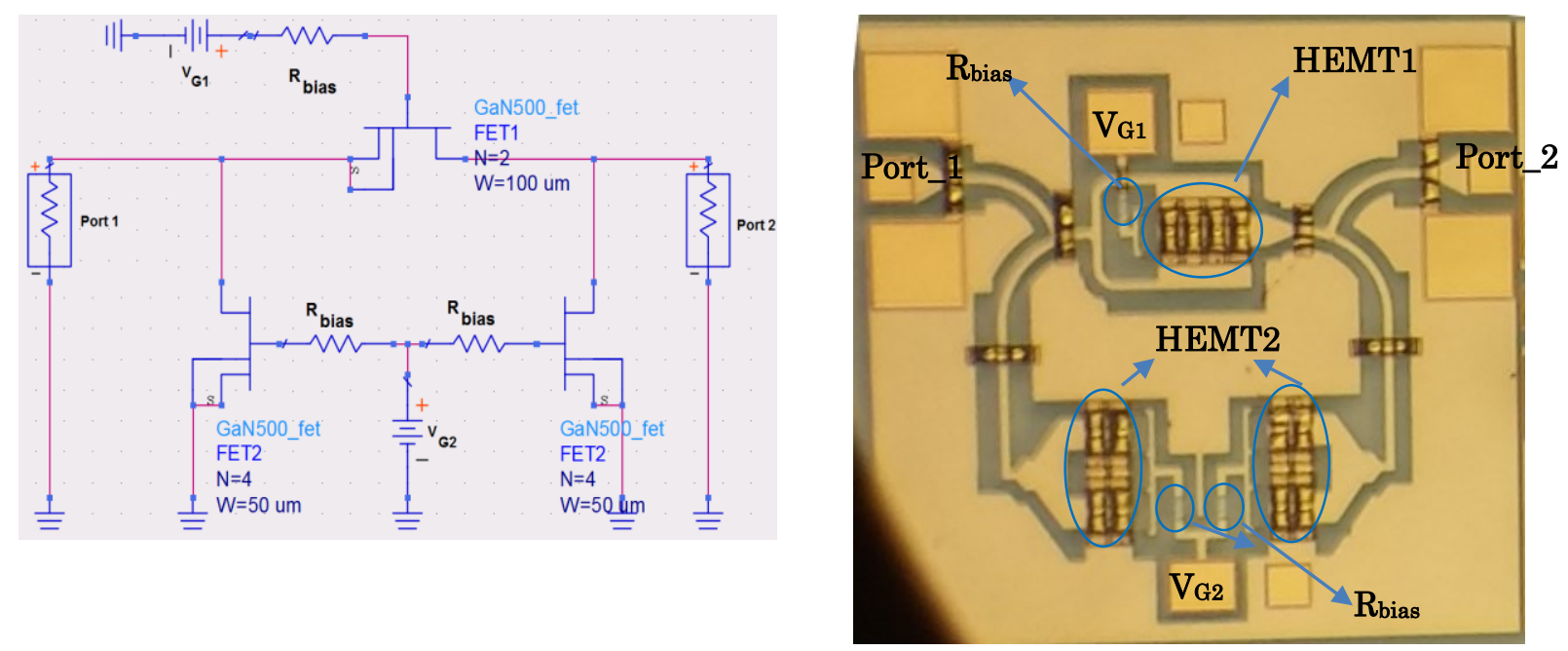

Figure III.54: Schematic (left) and Layout (right) of the Proposed SPST Switch Structure

As can be seen from Figure III.55, the isolation has been improved compared to the isolation of a $4 \times 50$ standalone switch (see Figure III.48). Up to 10 $\mathrm{GHz}$, an isolation of $-20 \mathrm{~dB}$ is still achievable while with $4 \times 50$ single switch an isolation of $-10 \mathrm{~dB}$ was achieved at $3 \mathrm{GHz}$. In addition, the insertion loss value achieved by this structure is better than $-3.5 \mathrm{~dB}$ up to $3 \mathrm{GHz}$ and then starts degrading. However, the insertion loss of $2 \times 100$ single switch was better than $-2 \mathrm{~dB}$ up to $5 \mathrm{GHz}$ (Figure III.47).

Comparing to the $2 \times 100$ standalone device, the isolation is improved while the insertion loss slightly dropped by $-1 \mathrm{~dB}$. With this proposed topology, it is still a challenge to meet good performances at higher frequency. Another topology based on SPDT is proposed to improve again the switch performances. 


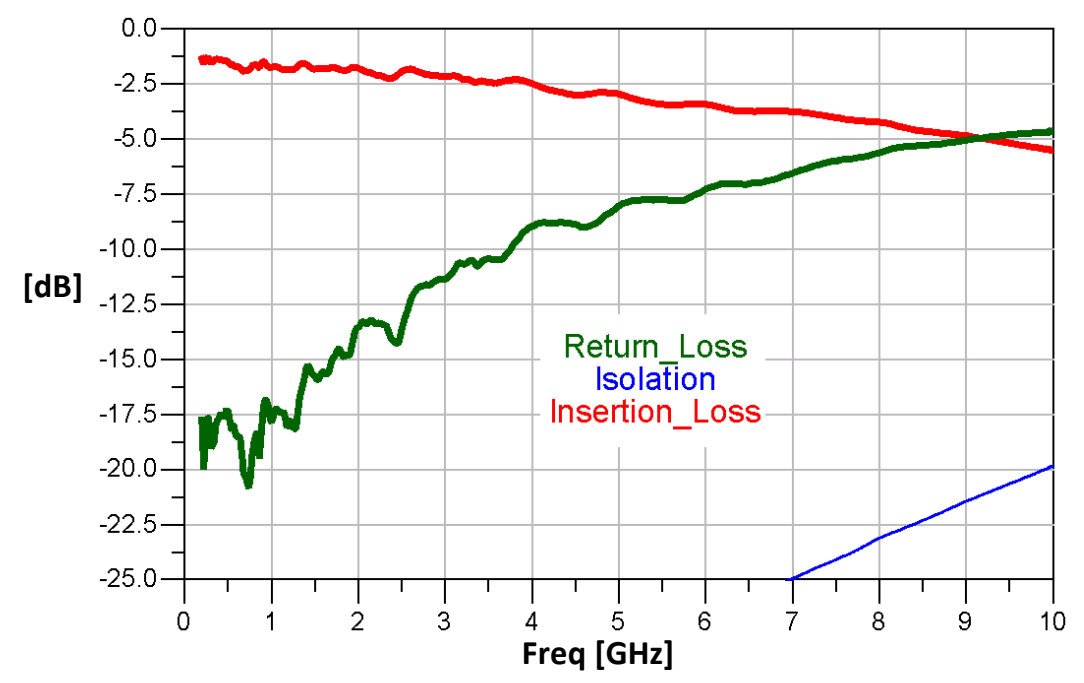

Figure III.55: Measured SPST performances

\subsection{SPDT Switch Structure}

A SPDT switch topology is proposed in order to improve the isolation at high frequencies. FETs are arranged in both series and shunt configurations. The series FETs provide a through-path for the ON-state, while the shunt FETs provide isolation for the OFF-state. The operation of the switch requires that series FETs and shunt FETs associated with each switch state have opposite (or complementary) conduction states and therefore opposite (or complementary) gate biases. Figure III.56 illustrates the SPDT topology. If the Port_1 to Port_2 path is ON and the Port_1 to Port_3 path is OFF, then FET1 and FET4 are biased ON, while FET2 and FET3 are biased OFF.

When the transistor FET1 as well as the FET4 are in the ON state corresponding to $\mathrm{V}_{\mathrm{G} 1}=0 \mathrm{~V}$ and $\mathrm{V}_{\mathrm{G} 2}=-5 \mathrm{~V}$, FET1 behave as a small resistor which allowing to the RF signal to go from port_1 to port_2. Verse-versa for the second state when the signal is from port_1 to port_2 

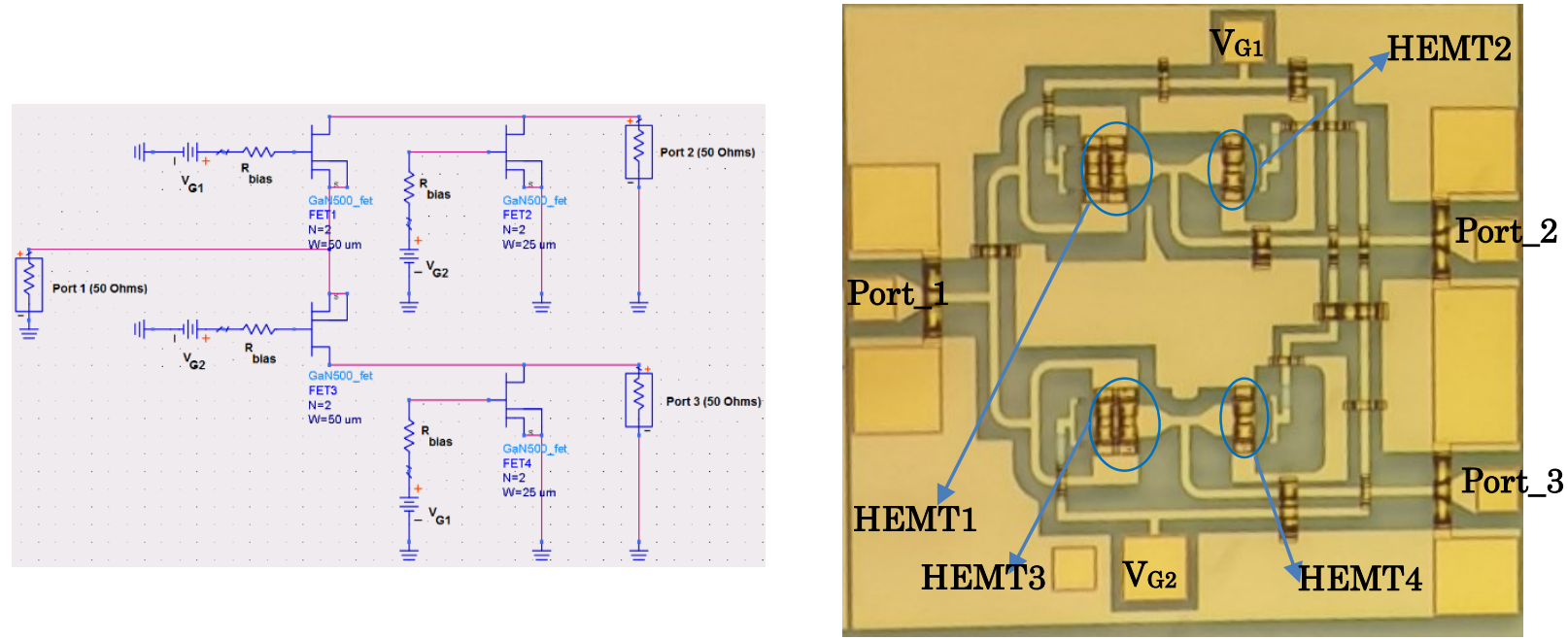

Figure III.56: Schematic (left) and Photograph (right) of the Proposed SPDT Switch Structure

As was expected with this topology, some improvement in isolation is achieved compared to SPST, while the insertion loss is increased by almost $1 \mathrm{~dB}$. For example, at $3 \mathrm{GHz}$ an isolation of $-25 \mathrm{~dB}$ and an insertion loss of $-3 \mathrm{~dB}$ were achieved. Unfortunately, the SPDT structure performances degrade with frequency. As we hope to go higher in frequency, around $10 \mathrm{GHz}$, with these realized switch topologies it will be impossible to achieve good performance. So, the SPDT structure which presents good overall performances compared to SPST will be used in a beam switched antenna system at $2.45 \mathrm{GHz}$. At this frequency, an isolation better than -25 $\mathrm{dB}$ and an insertion loss of $-3 \mathrm{~dB}$ are possible.

Due to the unavailability of having large signal equipment and also a $\mathrm{RF}$ probe of type GSGSG to measure the outputs of the SPDT either at IETR (University of Rennes 1) or DOE (Carleton University), we could not do the large signal measurement of this structure. However, as will be presented in Chapter IV (Section V), once this SPDT structure was mounted over a PCB by using wire-bonding technique, the large signal measurement have been conducted and results are presented and discussed in Chapter IV. In addition, by using ADS the SPDT small 
signal model can be built based on the small signal model of a single standalone switch. Here as shown in Figure III.57, only small signal measurements of this structure are reported.

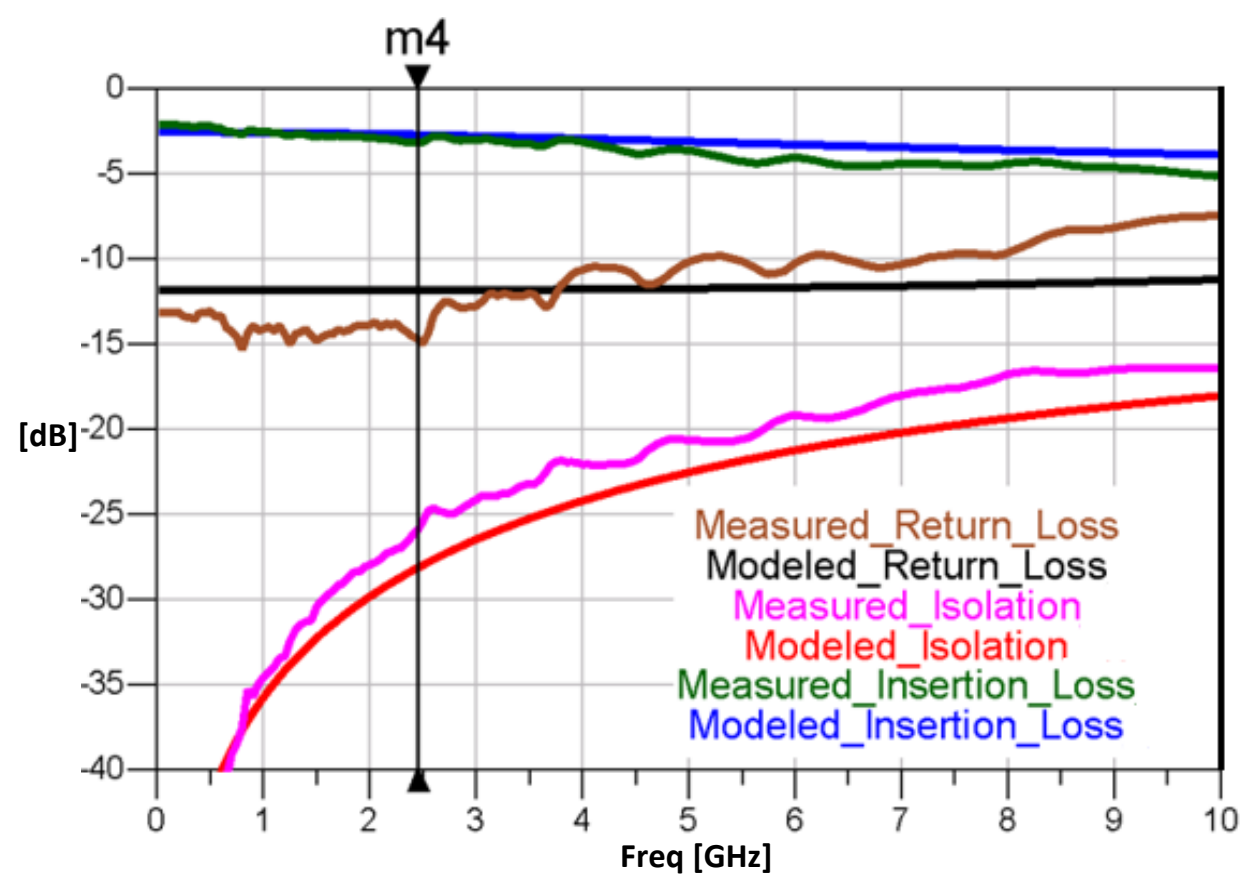

Figure III.57: Measured and Modeled Return Loss, Isolation, and Insertion Loss of SPDT Switch Structure

Table III.8: Performances of the SPDT at the Marker m4

\begin{tabular}{|c|c|}
\hline Performances & Values \\
\hline Modeled Insertion Loss & $\mathbf{- 2 . 7 2 3 ~ d B}$ \\
\hline Measured Insertion Loss & $\mathbf{- 3 . 1 6 4 ~ d B}$ \\
\hline Modeled Isolation & $\mathbf{- 2 8 . 1 5 8 ~ d B}$ \\
\hline Measured Isolation & $\mathbf{- 2 5 . 9 3 7 ~ d B}$ \\
\hline Modeled Return Loss & $\mathbf{- 1 1 . 8 4 7 ~ d B}$ \\
\hline Measured Return Loss & $\mathbf{- 1 4 . 6 9 7 ~ d B}$ \\
\hline
\end{tabular}




\section{Summary}

In this chapter, GaN HEMT-based varactor devices have been fully characterized and modeled for the first time, showing limited usefulness for high power applications due to their rapidly changing $\mathrm{C}-\mathrm{V}$ characteristic. These GaNbased varactors were designed in a standard GaN HEMT process, and were fabricated with two different gate lengths, $0.5 \mu \mathrm{m}$ (GaN500v1 and v2) and $0.15 \mu \mathrm{m}$ (GaN150v0). The varactor diode is realized by connecting together the drain and source terminations thus resulting in a single Schottky junction which can be considered as Planar Schottky Varactor Diodes (PSVDs). First of all, a small signal analysis has been conducted and by analyzing the extracted $\mathrm{C}_{\mathrm{Eq}}-\mathrm{V}_{\mathrm{G}}$ curves, mathematical expressions have been proposed to capture the nonlinear behavior that can be used to represent a variety of different GaN based varactor sizes. These equations maintain the simplicity of equivalent circuit modeling while offering good agreement with measurements. In addition, an equivalent simple circuit model was proposed and simulated, yielding good agreement between the simulated and the measured results. The obtained $\mathrm{Cmax} / \mathrm{Cmin}$ ratios and figures of merit compare favorably with recently reported GaN varactors.

Secondly, a general large signal model for GaN varactor diodes manufactured with gate lengths of $0.5 \mu \mathrm{m}$ (GaN500v2) has been presented. This model is based on an analytic expression which describes the $\mathrm{Ceq}-\mathrm{V}_{\mathrm{G}}$ curve and has shown to maintain a high level of accuracy, well into the large power range up to 18 $\mathrm{dBm}$. In addition, the proposed circuit model in small signal analysis is always valid for large signal by considering the new analytical equation of $\mathrm{C}_{\mathrm{Eq}}$ versus the input power $\left(\mathrm{P}_{\text {in. }}\right.$.) With reverse bias and $\mathrm{RF}$ power level included in the model, the junction capacitance ratio $\left(\mathrm{C}_{\mathrm{MAX}} / \mathrm{C}_{\mathrm{MIN}}\right)$ of the realized varactors was shown to be decreased from 1.73 to 1.25 . This ratio can be much further decreased, if the RF power level is increased further. From the presented results and given GaN's high breakdown voltage, these varactors would be very useful in various applications in receive mode, 
where power limiters could be completely excluded as protection circuitry from jammer signals.

Finally, in this chapter, different sizes single standalone switches and switch structures such as SPST and SPDT were fabricated and presented. As mentioned, only small signal measurement were reported. It was observed that, with single transistor in common gate configuration, the isolation is good at low frequencies but quickly drops with increasing frequency. In addition, it was observed that more the device is large, lower the insertion loss is achieved but poorer the isolation will be. A trade-off between insertion loss and isolation has been made to choose the single transistors that will be used for designing switch structures such as SPST and SPDT which could improve the performances at high frequencies. Comparing to the standalone device, it was revealed that with SPST structure, the isolation is improved while the insertion loss slightly dropped by $-1 \mathrm{~dB}$. With this proposed topology, it is still a challenge to meet good performance at our higher frequency. Another topology based on SPDT was proposed to improve more the performances. As was expected with this topology, some improvement in isolation is achieved compared to SPST, while the insertion loss is increased by almost $-1 \mathrm{~dB}$. For example, at $2.45 \mathrm{GHz}$ (frequency of interest) isolation better than $-25 \mathrm{~dB}$ and an insertion loss of $-3 \mathrm{~dB}$ were achieved. However, the SPDT structure performances degrade with frequency. Moreover, based on these small signal measurements, a model was introduced. It was observed that this linear model is the same to the one proposed in [[90] by Tyler Ross using GaN500v1 from NRC foundry. In addition, the fabricated SPDT switch structure will be integrated with antenna system to realize and to demonstrate the ability of using GaN devices for a beam switching antenna array. 


\section{Chapter IV}

\section{Realized GaN-Based Reconfigurable Circuits}

\section{Introduction}

As a result of the emerging need for multi-band and multi-services requirements within a single design structure, the electronically tunable or reconfigurable devices have experienced [92], over the last years, with a significant growth in radio frequency ( $\mathrm{RF}$ )/Microwave wireless front-end applications such as antennas, power amplifier, phase-shifters, oscillators and filters. Moreover, this has been the modern trends in technological of the new compact-structured RF transceivers [93].

Also, the use of wireless devices has increased dramatically over the last years. This increased utilization has led to a need for more efficiency RF circuits with 
more functionalities. Therefore, the continuing demands for highly portable, more reliable and more power efficient, low cost wireless systems require that future wireless devices must be compatible with different communication protocols and capable of operating over a wide frequency range. Furthermore, applications of such systems in personal and satellite communications impose the specifications for circuits miniaturized in size and weight. Thus, as wireless devices become more and more compact, the appeal of reconfigurable circuits is that they can decrease the number of components with identical specifications, decrease the number of input/output pins, reduce the size of the integrated circuit area, and reduce power consumption [94].

Tunable devices with a high performance are, also, more crucial for mobile devices and are for selective applications. Generally, an adaptive RF circuit must have the capabilities to handle different functionalities such as signal routing, impedance matching agility, adjustable gain amplification, tunable phase shifting [95], etc. In contrast, a tunable circuit with embedded tuning elements can carry out a switching function of several parallel circuits with a simple change to one of the elements. Figure III.1 illustrates this example for the case of duplexers in a tri-band system where a single tunable duplexer can replace three fixed duplexers [96].
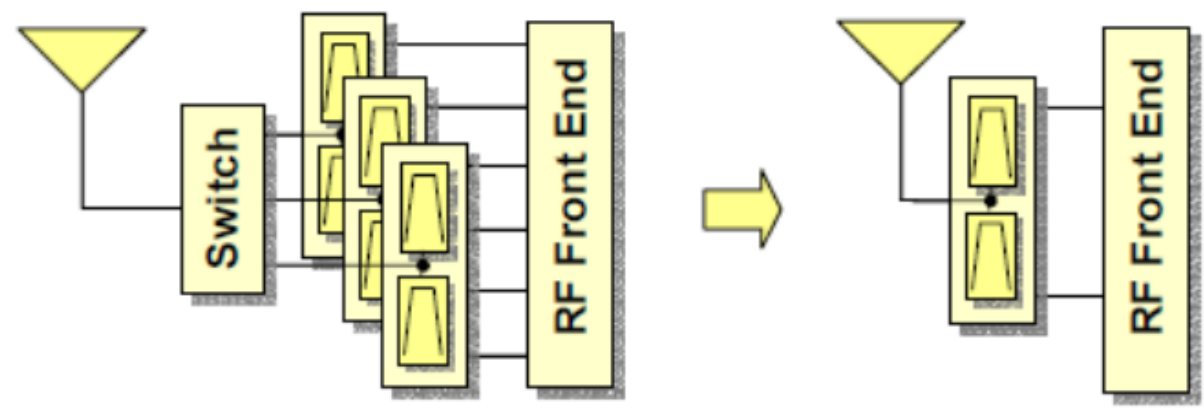

Figure IV.1: (left) Tri-band system with three fixed duplexers and (right) with a single tunable duplexer [96] 
Comparing the above architectures, a single tunable duplexer offers higher flexibility, more functionalities, lighter weight and denser integration. In addition, such a circuit tuning allows an easier controlling of systems through baseband signal processing, which can add attractive features of smartness of combined multiservices. Therefore, it is obvious that due to the convergence towards the integration of multiple standards and services into a single circuit and maintaining their expected performances at various configuration parameters, wireless systems are becoming more complex and highly smart.

Depending on the specific RF design where reconfigurable components are suitable, the result may be a saving in cost, reduction in size, better performance, and/or increased functionality. In order to implement these electronically reconfigurable components, there are currently various main techniques [95] that could be listed as follow:

- Ferrite materials

- Ferroelectric technology

- Semiconductor varactor diodes or switches

- Micro-electromechanical systems MEMS

During the last years, Monolithic Microwave Integrated Circuits (MMICs) based on semiconductors technology have become more attractive for electronic reconfigurability due to their small size, easiness integrations, possibly to be massproduced, etc. Most MMICs currently in production, such as GaAs, SiC, InP, etc, operate in the microwave and $\mathrm{mm}^{-\mathrm{w}}$ range with modest breakdown voltage and limited power handling capabilities. But there are increasing applications in the $\mathrm{RF} /$ microwave spectrum with higher power requirement.

As already presented in chapter II, GaN technology can be ideal for the design of high power devices for microwave and $\mathrm{mm}^{-\mathrm{w}}$ applications. In-depth research studies have been conducted to explore the performance of GaN technology, as well as reconfigurable RF/microwave circuits using GaN devices [97]. GaN HEMTs 
semiconductor devices are no longer limited to RF power amplifier applications, having been emerged to other applications such as LNAs, VCOs, mixers, and phase shifters.

For the above mentioned strong points of reconfigurable components, GaN technology can be considered as a real promising candidate semiconductors technology, capable of significantly reducing the number of components with identical specifications, size and cost of devices utilized in transmitter and receiver chains of front-end modules used in RF/microwave systems.

\section{Tunable Phase Shifters}

\section{Review}

Phase shifters are important components that find their use in many RF/microwave systems. For example, they can be used in electronic beam-scanning phased arrays, microwave instrumentation and measurement systems, power divider, and as modulators [97] in communication sub-systems.

Phase shifters can be analog or digital. Analog phase shifters provide a continuously variable phase, which can be controlled by a voltage. Electronically controlled analog phase shifters can be realized with varactor diodes that change capacitance with voltage, or nonlinear dielectrics. In the other hand, digital phase shifters provide a discrete set of phase states that are controlled by two-state "phase bits". They are characterized by a certain number of bits. This number defines straightforwardly the achieved phase. More bits there are, smaller phase step will be.

For example, a five bits phase shifter would have a $11.25^{\circ}\left(=\frac{360^{\circ}}{2^{5} \text { bits }}\right)$ as a phase shift step. This means that each bit allows to get a phase shift successively over $11.25^{\circ}$, $22.5^{\circ}, 45^{\circ}, 90^{\circ}$ and $180^{\circ}$. 
There are various design parameters that should be considered such as frequency range, total phase variance $(\Delta \phi)$, phase resolution, insertion loss, bandwidth, return loss, harmonics level, power handling and design area. This makes some challenges in the design and optimization of integrated phase shifters. In addition, there are five main phase shifter topologies and described as follow [98]:

- Switched line phase shifter

- Loaded line phase shifter

- Reflection type phase shifter

- All pass network phase shifter

- High Pass / Low Pass phase shifter

As highlighted in the $\mathrm{PhD}$ dissertation [[30] about GaN phase shifters, if phase shifters are implemented in a low power semiconductor technology, such as $\mathrm{Si}$ or GaAs, it may have to be placed in transmit and receive chains in RF front-end system, where it will not be damaged by large signals in any unexpected situation, thus resulting in relatively high cost and low integration density. Figure IV.2 shows a simplified RF front-end for a beamforming system. However, with recent developments in high power technology semiconductors such as GaN, it is possible to design phase shifters that can simplify the front-end system and make it more efficiently. An example of this is shown in Figure IV.3. Since the active devices in the phase shifter can handle both weak and strong signals, the circuit will operate properly and will not be damaged. As it is in the common path, only one phase shifter per antenna element is required as well, and the transceiver component count and cost can be drastically reduced. GaN-based Phase shifter with good high power handling and low loss hold the key to realize an affordable electronically steerable system, eliminating the need for active transceiver modules at every element. 


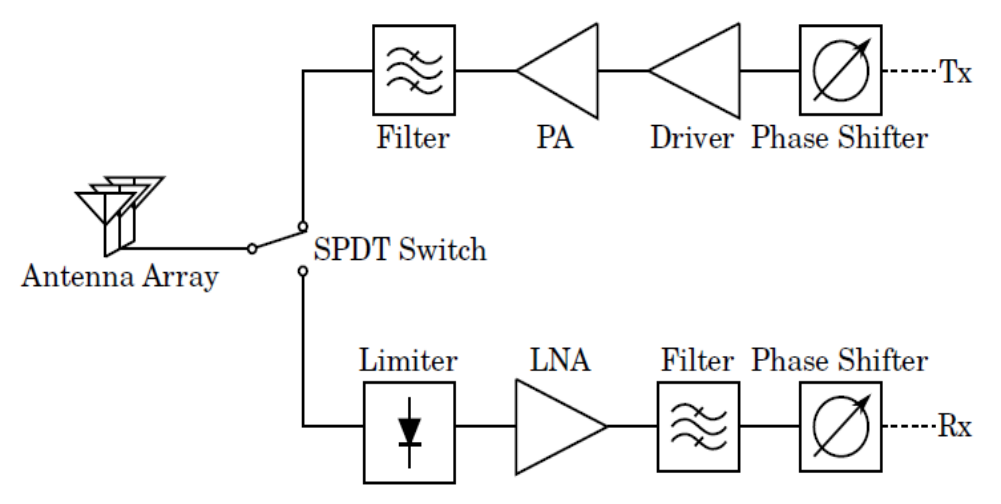

Figure IV.2: Simplified RF front-end for a typical beamforming system in a nonGaN technology [[30]

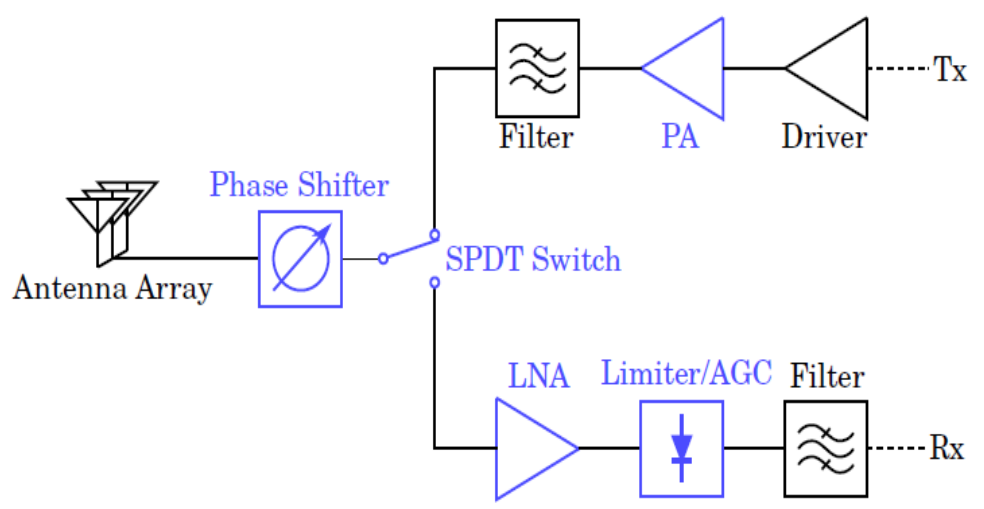

Figure IV.3: Example RF front-end possible with GaN with key components in blue [[30]

While there has been significant progress made in developing GaN technology for high-power amplification applications as explained before in Chapter II, there have been few studies to date on the use of GaN HEMTs for high-power $\mathrm{RF} /$ microwave phase shifters applications. In future $\mathrm{RF} /$ microwave systems that are based on GaN device technology, the GaN control elements, such as phase shifters, will often be conjointly placed together with high-power GaN amplifiers, thereby requiring an in-depth look at these control devices in term of their parameters design presented previously. 
Using GaN technology, a few phase shifters works have already been reported over the last few years. A new type of GaN HEMT based high power shifter at X-band permitting to switch between two states [99] was reported for the first time by a research group from Carleton University. As shown in Figure IV.4, this work is based on the high-pass/low-pass phase shifter or T-topology which has original advantage such as its superior power and phase-bandwidth capabilities compared with more conventional switched line, reflection, or loaded line phase shifters. As illustrated, the proposed phase shifter consists of two SPDT switches, a low pass network, and a high pass network. The $0 \% 45^{\circ}$ signal shift is achieved by switching between two filters, low pass and high pass networks. As shown in Figure IV.5 and Figure IV.6, where measured results are depicted, the proposed $0^{\circ} / 45^{\circ}$ high-pass/lowpass phase shifter exhibits low insertion loss $(2.5 \mathrm{~dB})$, good return loss, and amplitude variation lower than $0.5 \mathrm{~dB}$ for the two phase states over the entire operational bandwidth ranging from 6 to $13 \mathrm{GHz}$, while the measured phase difference between the two states varies from $45^{\circ}$ to $55^{\circ}$ over the design frequency range of 8-12 GHz (see Figure IV.7). This work has been demonstrated by using a $0.8 \mu \mathrm{m}$ gate GaN HEMT process from NRC foundry which is the first GaN HEMTs NRC developed process.

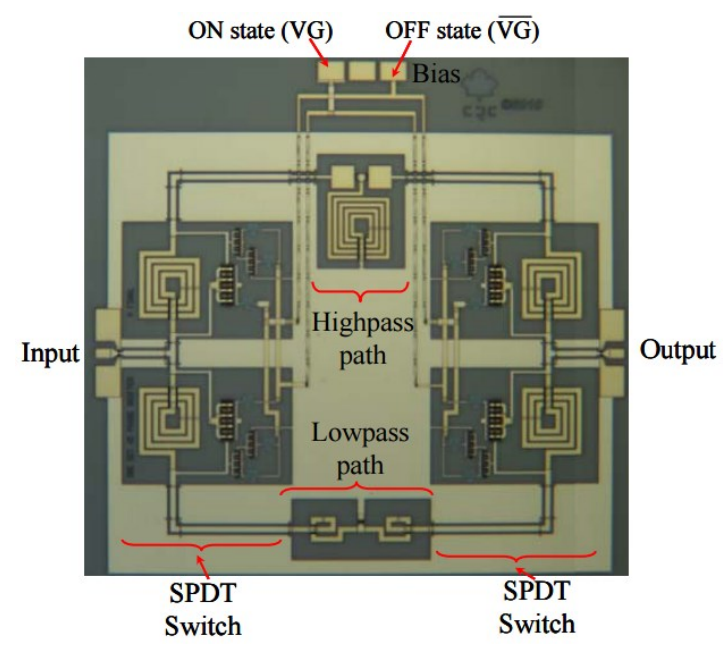

Figure IV.4: Photograph of a novel GaN HEMT based high power high-pass/lowpass phase shifter [99] 


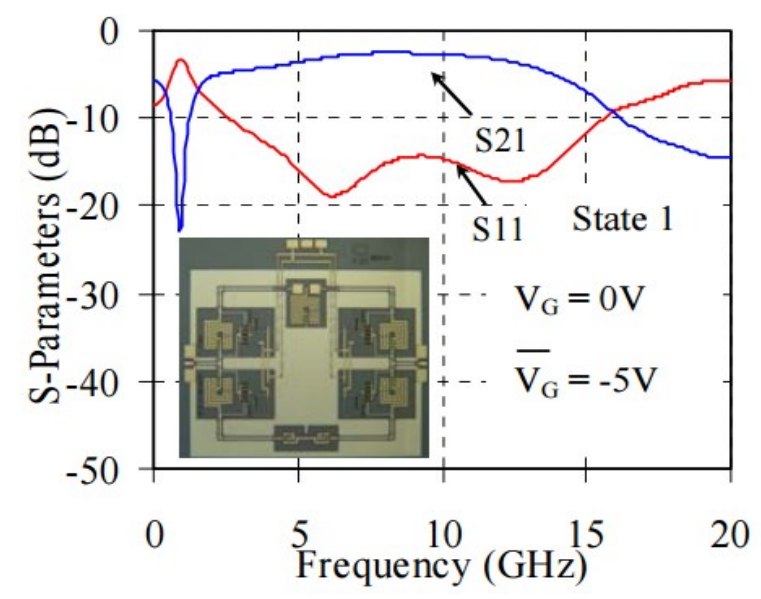

Figure IV.5: Measured insertion and return loss for high pass [99]

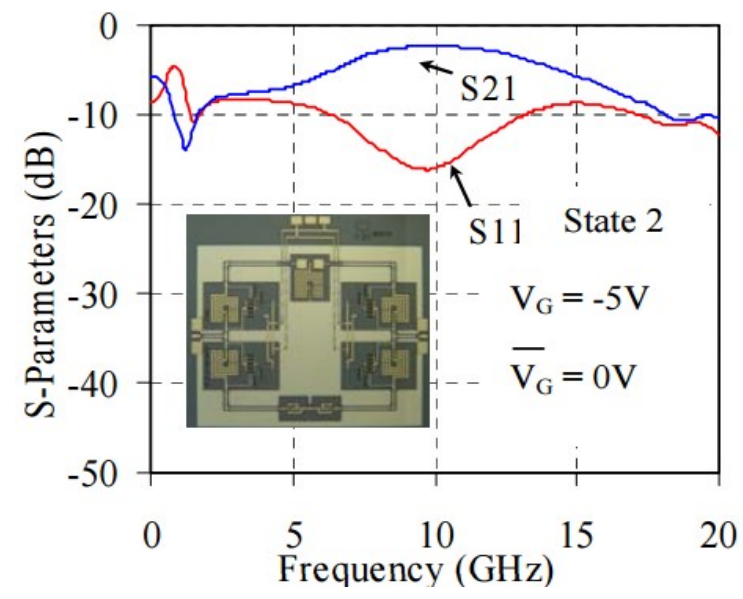

Figure IV.6: Measured insertion and return loss for low pass [99]

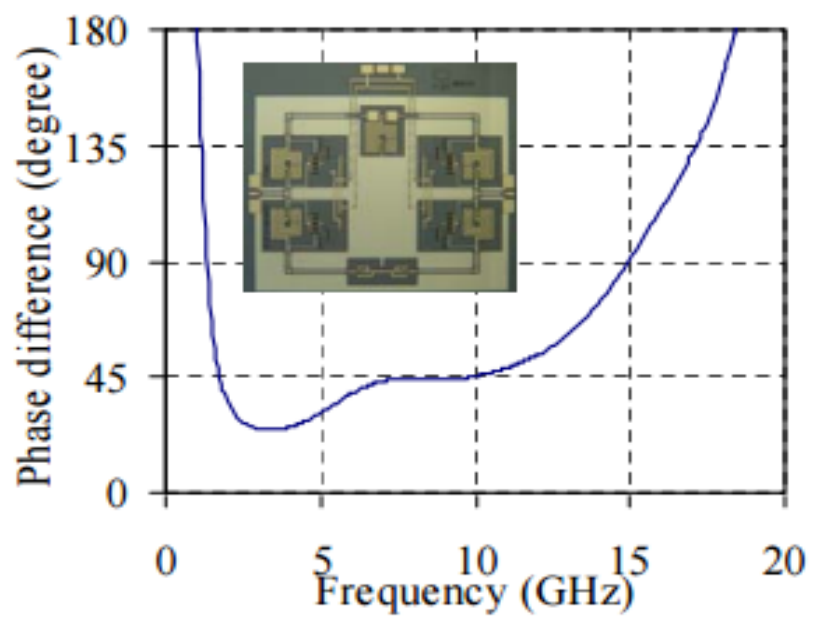

Figure IV.7: Measured phase difference between the two states [99] 
Later on 2014, the same authors have reported a novel high power X-band GaN phase shifter [100] based on switched-filter configuration by using a novel developed NRC GaN fabrication process which is a $0.5 \mu \mathrm{m}$ gate width. The use of this topology is widely found useful when compact circuits are required because less chip area is required compared with other phase shifter topologies. Figure IV.8 shows a microphotograph of the designed phase shifter. The manufactured $0^{\circ} / 22.5^{\circ}$ switched filter phase shifter has much wider bandwidth covering 8-16 GHz, while maintaining low insertion loss of $2 \mathrm{~dB}$, good return loss $>11.15 \mathrm{~dB}$ and an amplitude imbalance of less than $1.03 \mathrm{~dB}$ across X-band. Figure IV.9 presents the obtained phase shift while the measurements of linearity and power handling capability of the circuit are depicted in Figure IV.10. With the $0^{\circ}$ (bypass) state, the measured IIP3 was $46.2 \mathrm{dBm}$ while for the $22.5^{\circ}$ phase shift state had a higher IIP3 of $50.4 \mathrm{dBm}$. The obtained 1 $\mathrm{dB}$ compression point is well over $40 \mathrm{dBm}$.

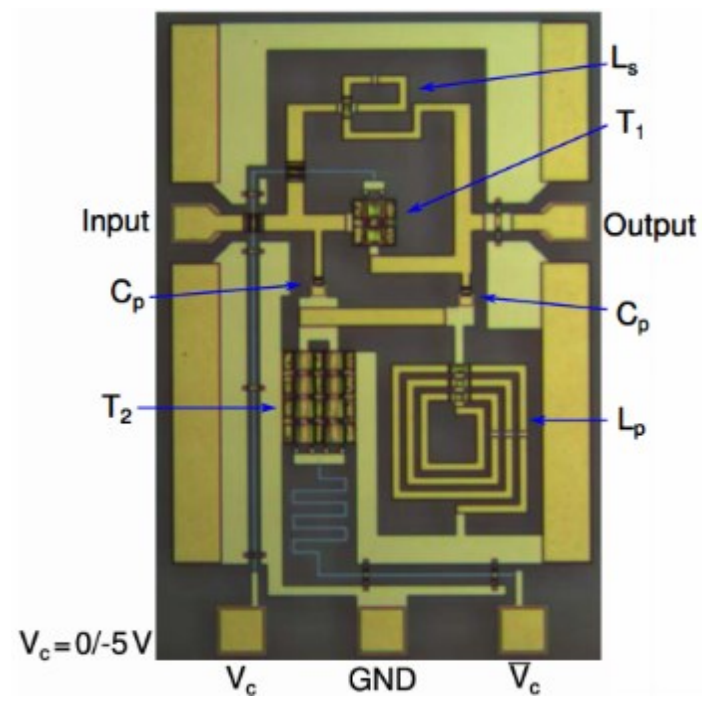

Figure IV.8: Microphotograph of the $0^{\circ} / 22.5^{\circ}$ GaN switched-filter phase shifter [100] 


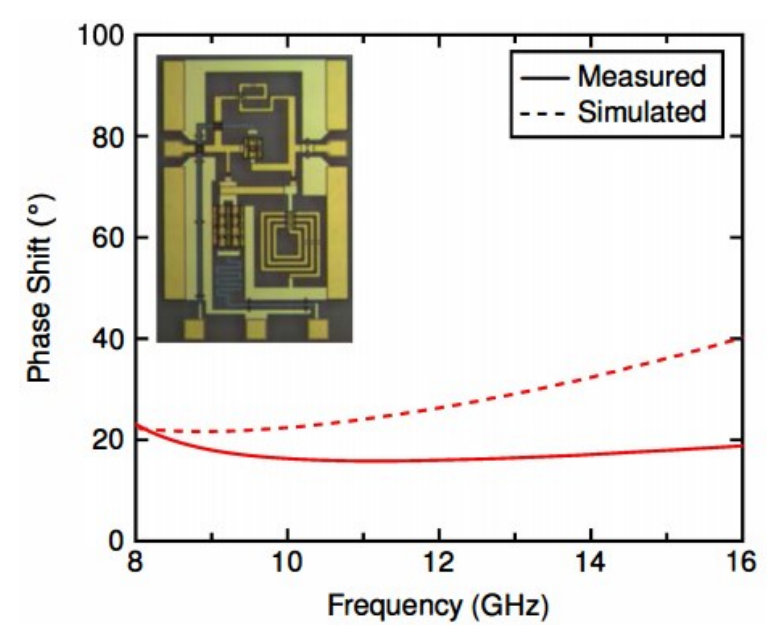

Figure IV.9: Measured and simulated phase shift [100]

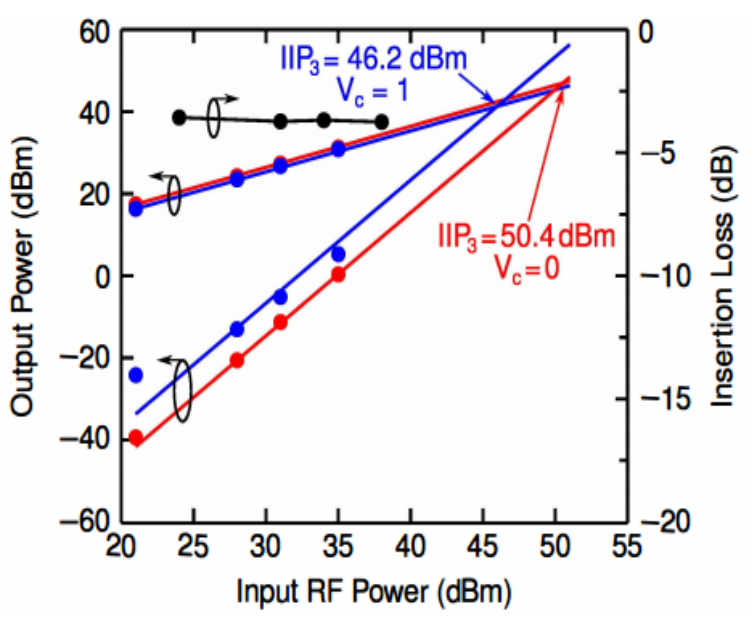

Figure IV.10: Linearity measurement [100]

Using the topology presented in [[30], an analog phase shifter using GaN Schottky diode is reported in [101] for $35 \mathrm{GHz}$ operation. This design reports fabrication and experimental characteristics of a $35 \mathrm{GHz}$ MMIC analog phase shifter using GaN Schottky diodes to enable continuous phase shifting. Figure IV.11 shows the schematic and photograph of the designed T-phase shifter. The reported design contains two series connected diodes and another diode in the shunt arm which is connected to a spiral inductor to achieve the optimum matching and phase shifting at $35 \mathrm{GHz}$. The phase shifter presents analog phase shifting capability up to $45^{\circ}$ with $\sim 7 \mathrm{~dB}$ insertion loss and a return loss of $-10 \mathrm{~dB}$ in the 32 to $38 \mathrm{GHz}$ range as 
illustrated in Figure IV.12. Good power handling capability and robust performance is demonstrated through large signal analysis and experimental characterization as explained in this paper.

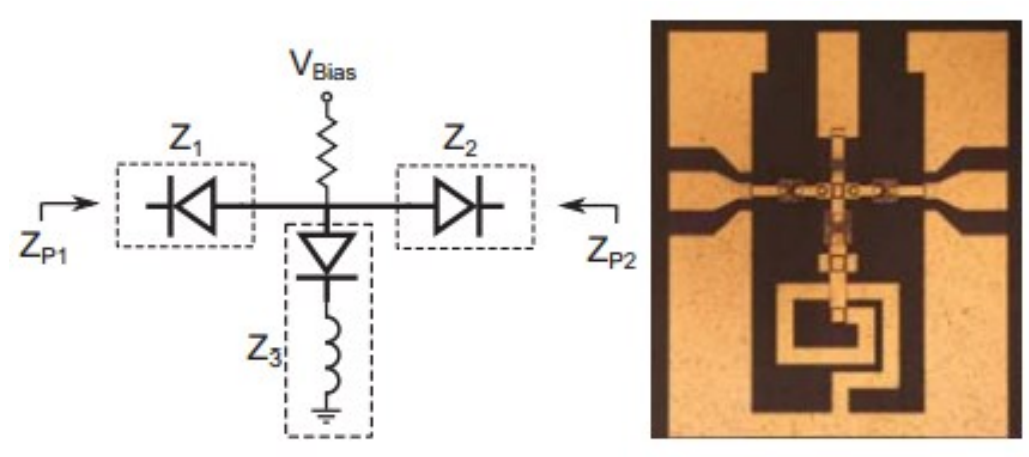

Figure IV.11: T-Phase shifter Circuit Configuration and microscope image [101]

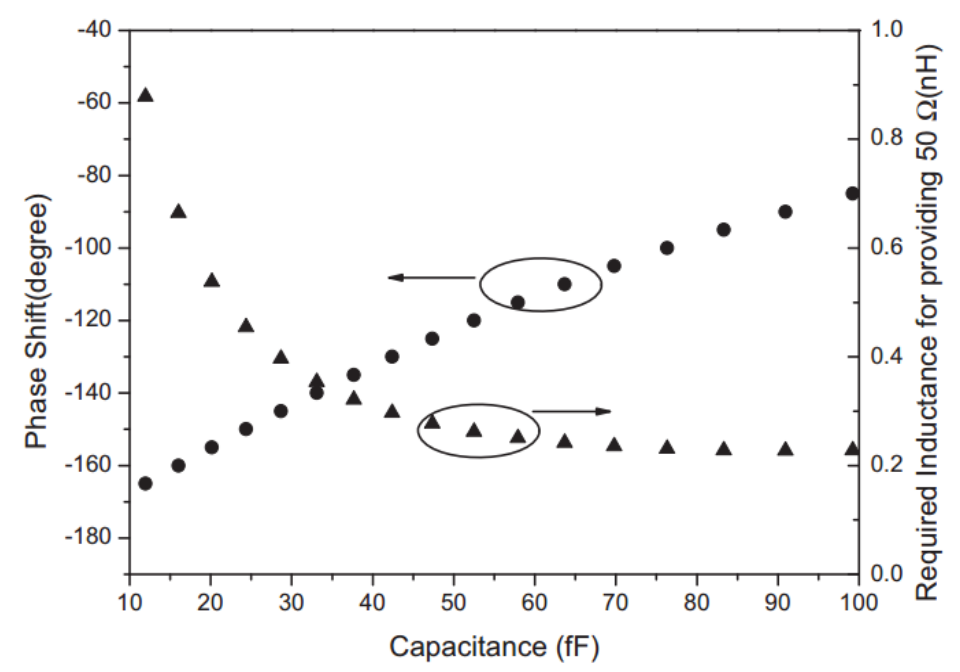

Figure IV.12: Phase shift of the high-pass T-network and the corresponding inductance for $50 \Omega$ impedance matching [101] 


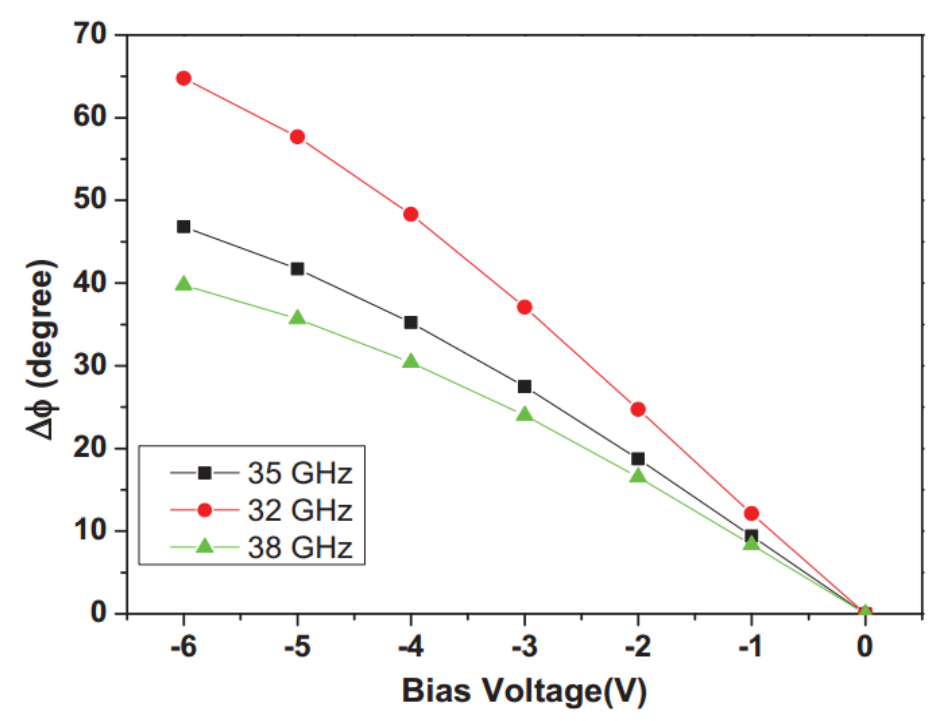

Figure IV.13: phase shift of the T-Phase shifter in the frequency range of $32 \mathrm{GHz}$ to $38 \mathrm{GHz}[101]$

\section{Design of Analog GaN-based Phase Shifter}

Analog phase shifter based varactor diodes structure is, first of all, very simple to design. Secondly, compared to a digital MMIC phase shifter, the power consumption is low and only a single input control voltage is required. Additionally, a continuous phase shift is easily achievable, thus allowing to provide any desired phase shift. Among the more useful topologies for analog phase shifters are the loaded line structure and the reflective structure employing quadrature $3-\mathrm{dB} 90^{\circ}$ hybrid coupler using either lumped or distributed elements with two reflective loads at terminations. One of the major drawbacks of reflection phase shifter structure is that the overall insertion loss of the circuit is usually high. These insertion losses are especially related to the equivalent series resistor presented by the used varactor and also the coupler's elements.

Using the first commercialized NRC GaN150v0 HEMTs process, a phase shifter based on quadrature reflection structure was designed and simulated using ADS Agilent simulator, along with the development of the reported small signal GaN 
varactor diodes model. Here this developed GaN varactor diode, already presented in Chapter III, is used as tuning load purpose. Moreover, at least two varactor diodes are required to achieve a theoretical phase shift of $180^{\circ}$ [102], making the device smaller and less expensive to fabricate compared to digital phase shifter; and the diodes are operated at reverse bias, having very fast response times and requiring voltage but no current consumption.

It is highly import to keep in mind that, to the author knowledge's, this the first developed GaN phase shifter based on quadrature reflection structure even if only simulation results will be presented.

Here, before presented the simulated results, a description of how this phase shifter structure works is giving. As shown in Figure IV.14 and Figure IV.15, basically, a phase shifter based reflection structure consists of a quadrature hybrid coupler that splits the incident signal into two separate signals of equal amplitude and $90^{\circ}$ phase difference over the two opposite ports (2 and 3), while isolating the adjacent one (port 4). These two signals reflect from the two tunable loads, GaN varactors connected to the coupled and thru ports of the hybrid, and add up in phase at the hybrid's isolated port if both of the loads are identical, and will ideally be isolated from the phase shifter's input signal. This means that the voltage at the output port can be written as [103]:

Equation (9)

$$
\text { Vout }=j . \Gamma . \text { Vin }
$$

Equation (10)

$$
\Gamma=|\Gamma| . e^{-j \phi}
$$

The phase angle, $\phi$, of the reflection coefficient, $\Gamma$, can be varied by changing the capacitance of the loading varactor diodes which are controlled by DC voltage and, theoretically, have to present a pure imaginary impedance. While $|\Gamma|$ 
must be unchanged and equal to 1 in order to not introduce any additional loss into the phase shifter. Practically, it is impossible to achieve perfect reflection from a load which means that some power will be dissipated in the load and $|\Gamma|$ will be less than one. Therefore, some loss will always occur between the input and output signals. By inserting varactors in the terminations, a continuous control of the phase shift is achieved in this manner.

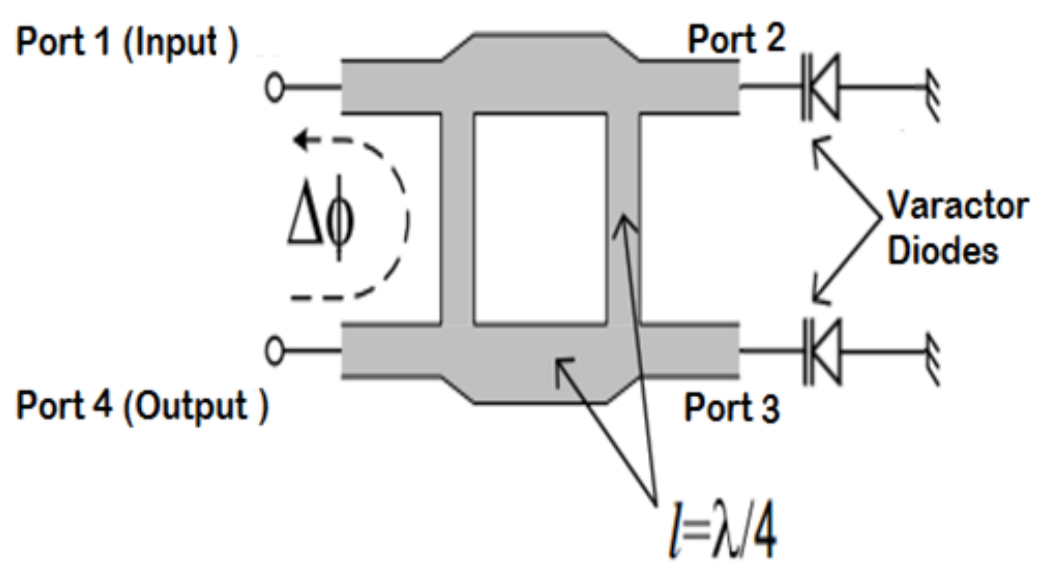

Figure IV.14: Phase shifter based on 3-dB/90० branch-line hybrid coupler using distributed elements

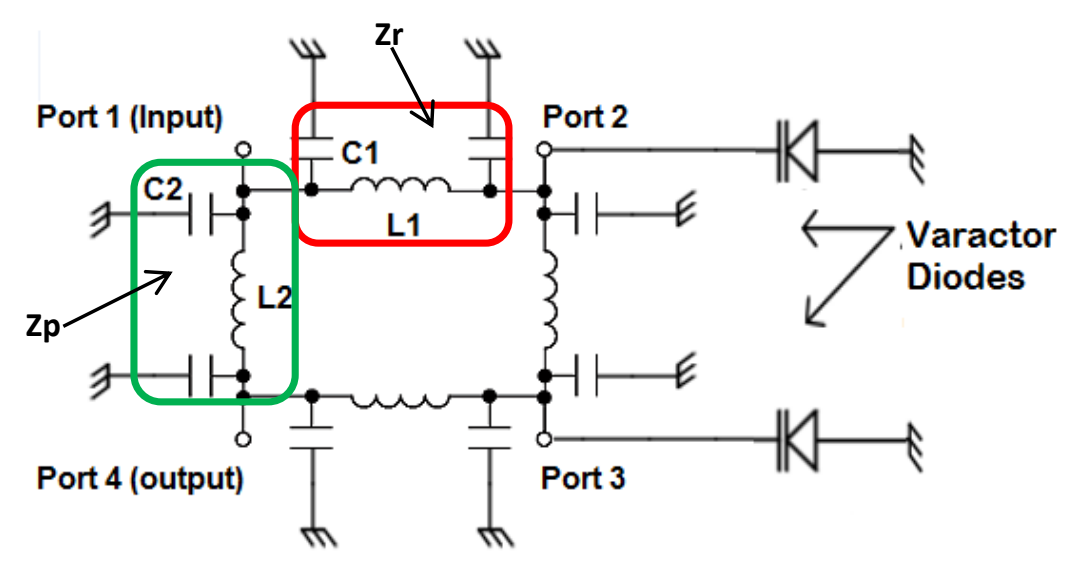

Figure IV.15: Phase shifter based on 3-dB/90० branch-line hybrid coupler using lumped elements 
To design this phase shifter topology, firstly, a 3-dB $90^{\circ}$ hybrid coupler has been designed and simulated. The simulated results will be discussed later in this section. Then, the two coupler's outputs ports have been loaded by GaN varactors.

The final phase shifter design presented in this section was designed and were planned to be manufactured by using GaN150v1 process. However, due to factors beyond our control, fabrication of this phase shifter at the CPFC-NRC could not proceed as planned. The design was planned to be submitted for fabrication on 7th September, 2015 and then four months later samples should be delivered. Since this design was the only application for $2 \times 2 \mathrm{~mm}^{2}$ space in this run fabrication referenced as " $1503 G K$ " that cost $\$ 70 \mathrm{~K} /$ run and because it is outside of our funds to purchase the entire run. The number of participants is not enough so the run of manufacturing has been completely cancelled. Due to this unexpected fabrication issue, only simulation results and design steps will be reported here

\subsection{Quadrature 3-dB 90 Hybrid Coupler}

Quadrature $3-\mathrm{dB} 90^{\circ}$ hybrid couplers are usually realized in some type of microstrip or stripline technology using distributed quarter-wavelength $(\lambda / 4)$ transmission lines (see Figure IV.14). They have been extensively progressed because of their good bandwidth of operation and of its straightforward design and reliability. However, this design approach could require several $\mathrm{mm}^{2}$ of area to be realized and renders their size relatively large even for $\mathrm{mm}$-wave frequencies due to the length of $\lambda / 4$ lines [104]. Thus it would consume considerable wafer space of the used semiconductor technology, and the design size gets much larger. Additionally, the most important issue of the distributed $\lambda / 4$ hybrid coupler in MMIC applications is the chip area which is directly related to the chip cost. Also the most significant challenge when dealing with a priced semiconductor technology especially GaN HFET technology is the high expense costs for technology access. Hence, there have 
been several attempts to reduce the size of coupled line coupler, the most important alternative solution is to use lumped elements instead of resonant $\lambda / 4$ transmission lines [104]. However, the bandwidth of the structure hybrid coupler is usually narrower than that of the typical $\lambda / 4$ structure. Another issue comes up is that this structure becomes inefficient at high frequency (i.e $\mathrm{mm}^{-} \mathrm{w}$ ) because of the inductors become very lossy and also tend to resonate at frequencies very close to the operating frequencies.

In this approach, the quarter-wave lines are approximated at operating frequency with lumped elements as depicted in Figure IV.15. Theatrically, the ABCD matrix of a single segment representing a perfect transmission line characterized by a characteristic impedance of $\mathrm{Zr}$ is written as follow:

Equation (11)

$$
\left(\begin{array}{ll}
A & B \\
C & D
\end{array}\right)=\left[\begin{array}{ll}
\operatorname{Cos} \theta & \mathrm{j} Z \mathrm{r} \operatorname{Sin} \theta \\
\mathrm{j} \frac{1}{\mathrm{Zr}} \operatorname{Sin} \theta & \operatorname{Cos} \theta
\end{array}\right]
$$

The equivalent of this matrix by using lumped elements can be written as follow:

Equation (12)

$$
\left(\begin{array}{ll}
A & B \\
C & D
\end{array}\right)=\left[\begin{array}{ll}
1 & 0 \\
j \omega C 1 & 1
\end{array}\right]\left[\begin{array}{ll}
1 & \mathrm{j} \omega \mathrm{L} 1 \\
0 & 1
\end{array}\right]\left[\begin{array}{ll}
1 & 0 \\
\mathrm{j} \omega \mathrm{C} 1 & 1
\end{array}\right]=\left[\begin{array}{ll}
1-\omega^{2} L 1 C 1 & \mathrm{j} \omega \mathrm{L} 1 \\
\mathrm{j} \omega \mathrm{C} 1\left(2-\omega^{2} \mathrm{~L} 1 \mathrm{C} 1\right) & 1-\omega^{2} L 1 C 1
\end{array}\right]
$$

From Equation (11) and Equation (12), we can drive the following equations:

Equation (13)

$$
\operatorname{Cos} \theta=1-\omega^{2} L 1 C 1
$$


Equation (14)

$$
\mathrm{ZrSin} \theta=\omega \mathrm{L} 1
$$

Equation (15)

$$
\frac{1}{\mathrm{Zr}} \operatorname{Sin} \theta=\omega \mathrm{C} 1\left(2-\omega^{2} \mathrm{~L} 1 \mathrm{C} 1\right)
$$

The combining between Equation 14 and Equation 15 gives the following equation:

Equation (16)

$$
\frac{1}{\mathrm{Zr}}=\sqrt{\frac{2 \mathrm{C} 1}{\mathrm{~L} 1}-(\omega C 1)^{2}}
$$

So the passive elements, L1 and C1, of the hybrid coupler can be calculated by the following driven equations:

Equation (17)

$$
L 1=\frac{\mathrm{ZrSin} \theta}{\omega}
$$

Equation (18)

$$
C 1=\frac{1}{\omega \mathrm{Zr}} \sqrt{\frac{1-\cos \theta}{1+\cos \theta}}
$$

By using the same previous procedures, the other passives elements, L2 and $\mathrm{C} 2$, can be, also, calculated as follow:

Equation (19) 


$$
L 2=\frac{\mathrm{Zp} \operatorname{Sin} \theta}{\omega}
$$

Equation (20)

$$
C 2=\frac{1}{\omega \mathrm{Zp}} \sqrt{\frac{1-\cos \theta}{1+\cos \theta}}
$$

So these previous equations give theoretically first guess values of the lumped elements of hybrid coupler.

Based on this topology, we report here a simulation analysis of a new fully embedded lumped element GaN MMIC 3-dB/90 ${ }^{\circ}$ hybrid coupler at X-band that it will be later used to conduct an analysis of a phase shifter design. A three-dimensional layout view of the coupler, showing embedded metal and vias layers, of the designed coupler is displayed in Figure IV.20.

The built block of the $3-\mathrm{dB} / 90^{\circ}$ hybrid coupler using lumped spiral inductors and metal-insulator-metal (MIM) capacitors is very small in size $\left(0.85 \times 1 \mathrm{~mm}^{2}\right)$. It consists of lumped multi-turn spiral inductors and MIM capacitors from NRC GaN150 PDK components library [105]. The mutually coupled inductors turns are rectangular spiral inductor type and are implemented with reinforced between two metals, metal 2 (2ME) on metal 1 (1ME) by using via connection (VIA2), which results in an inductor having a higher $\mathrm{Q}$-factor and size reducing. The current carrying capability of this inductor is limited by the width of the air-bridges. Higher currents can be sustained by increasing the width of the air-bridges, with a commensurate increase in the gap spacing between the inductor turns. A sample layout for a square inductor is shown in Figure IV.16, while in Figure IV.17 an inductor equivalent circuit is presented which is used to estimate the inductance $\left(\mathrm{L}_{\mathrm{S}}\right)$, series resistance $\left(R_{S}\right)$ and first resonance frequency $F_{R E S}$.

The MIM capacitors are formed by $2 \mathrm{ME}$ on top of $1 \mathrm{ME}$ without a VIA2 connection. A layer of silicon nitride, also used to provide insulation, is used as the 
dielectric layer. The dielectric constant of the silicon nitride is 6.5 and the thickness is $0.3 \mathrm{um}$ [105]. The capacitance density of the MIM structure is $0.19 \mathrm{fF} / \mu \mathrm{m}^{2}$. Figure IV.19 illustrates the capacitor layout.

At $10 \mathrm{GHz}$, the optimized values of the four lumped elements, L1, L2, C1 and $\mathrm{C} 2$, constituting the hybrid coupler are, respectively, $6.7 \mathrm{nH}, 8.45 \mathrm{nH}, 369 \mathrm{fF}$ and $136 \mathrm{fF}$. These values, firstly, have been determined by using the above equations (Equation (17, Equation (18, Equation (19 and Equation (20) and then have been optimized in ADS to achievegood performances. So, these values are corresponding to parameters presented in Table IV.1 and Table IV.2.

Table IV.1: Optimized Values of Inductors L1 and L2

\begin{tabular}{|c|c|c|}
\hline & Inductor L1 & Inductor L2 \\
\hline Value $(\mathrm{nH})$ & $\mathbf{6 . 7}$ & $\mathbf{8 . 4 5}$ \\
\hline Ns $($ turns $)$ & $\mathbf{1 0}$ & $\mathbf{1 0}$ \\
\hline $\mathrm{L} 1(\mu \mathrm{m})$ & $\mathbf{1 2 0}$ & $\mathbf{1 5 5}$ \\
\hline $\mathrm{L} 2(\mu \mathrm{m})$ & $\mathbf{1 2 5}$ & $\mathbf{1 2 5}$ \\
\hline $\mathrm{L} 3(\mu \mathrm{m})$ & 110 & 135 \\
\hline $\mathrm{Ln}(\mu \mathrm{m})$ & $\mathbf{2 0}$ & $\mathbf{2 0}$ \\
\hline $\mathrm{W}(\mu \mathrm{m})$ & 15 & 15 \\
\hline $\mathrm{S}(\mu \mathrm{m})$ & $\mathbf{5}$ & $\mathbf{5}$ \\
\hline $\mathrm{Wb}(\mu \mathrm{m})$ & 10 & 10 \\
\hline $\mathrm{Ab}$ & 90 & 90 \\
\hline $\operatorname{Be}(\mu \mathrm{m})$ & $\mathbf{3 0}$ & $\mathbf{3 0}$ \\
\hline
\end{tabular}

Table IV.2: Optimized Values of Capacitors C1 and C2

\begin{tabular}{|c|c|c|}
\hline & Capacitor C1 & Capacitor C2 \\
\hline Value $(\mathrm{fF})$ & 369 & 136 \\
\hline W $(\mu \mathrm{m})$ & 43.86 & 26.63 \\
\hline $\mathrm{L}(\mu \mathrm{m})$ & 43.86 & 26.63 \\
\hline Wc $(\mu \mathrm{m})$ & 15 & 10 \\
\hline
\end{tabular}




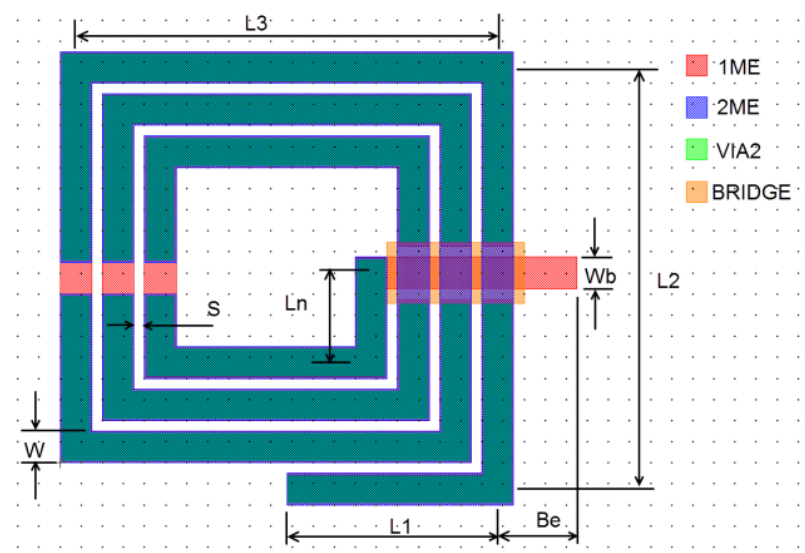

Figure IV.16: Layout of a Spiral Inductor Generated from NRC GaN150 PDK [105]

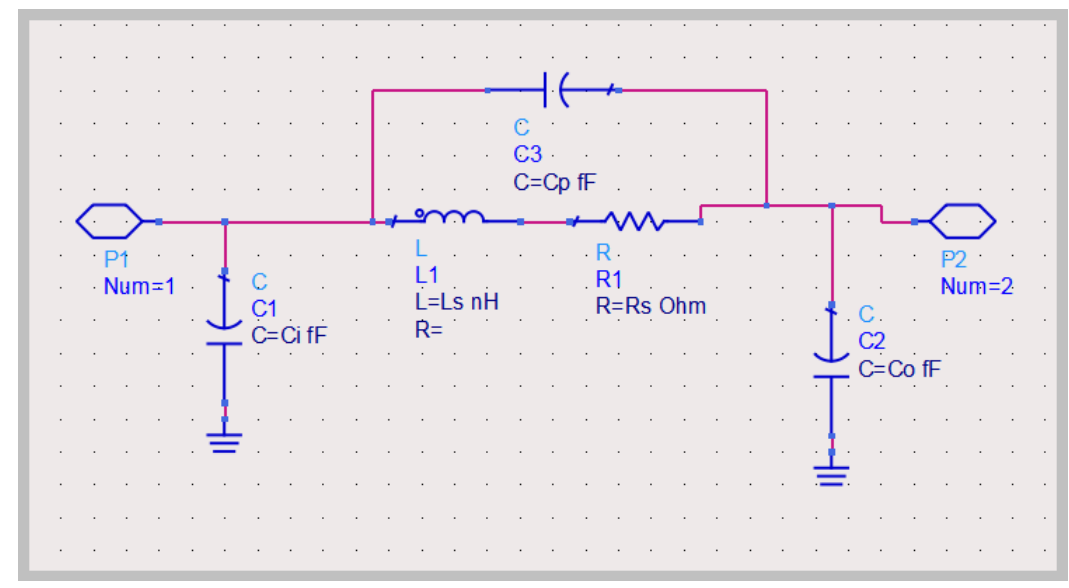

Figure IV.17: An Equivalent Circuit to Estimate LS, RS and Fres [105]

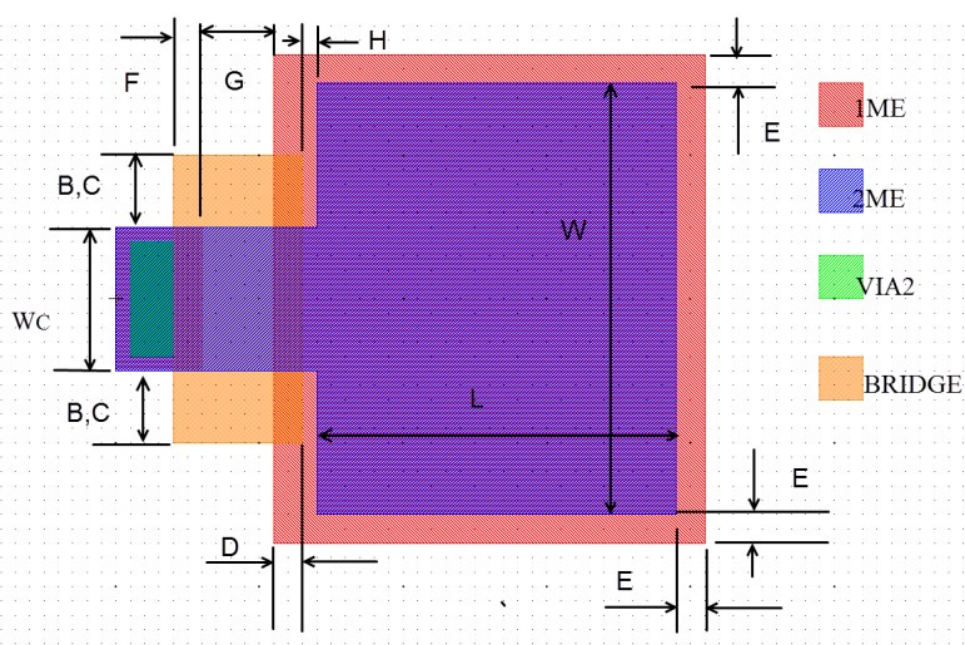

Figure IV.18: Layout of A MIM Capacitor [105] 
For the presented hybrid coupler, as indicated in Figure IV.19, port 1 is designed as the input port, port 2 as the transmitted port, port 3 the coupled port and port 4 the isolated port. Since this is a symmetrical network, any port can be used as the input port and the function of the other ports appropriately defined. Through wafer vias (TWV) are used for connection to the backside ground wafer metal. The ports 1 and 4 consist of G-S-G pads that have a pitch of $150 \mu \mathrm{m}$ to be compatible with $\mathrm{RF}$ probes of the same geometry.

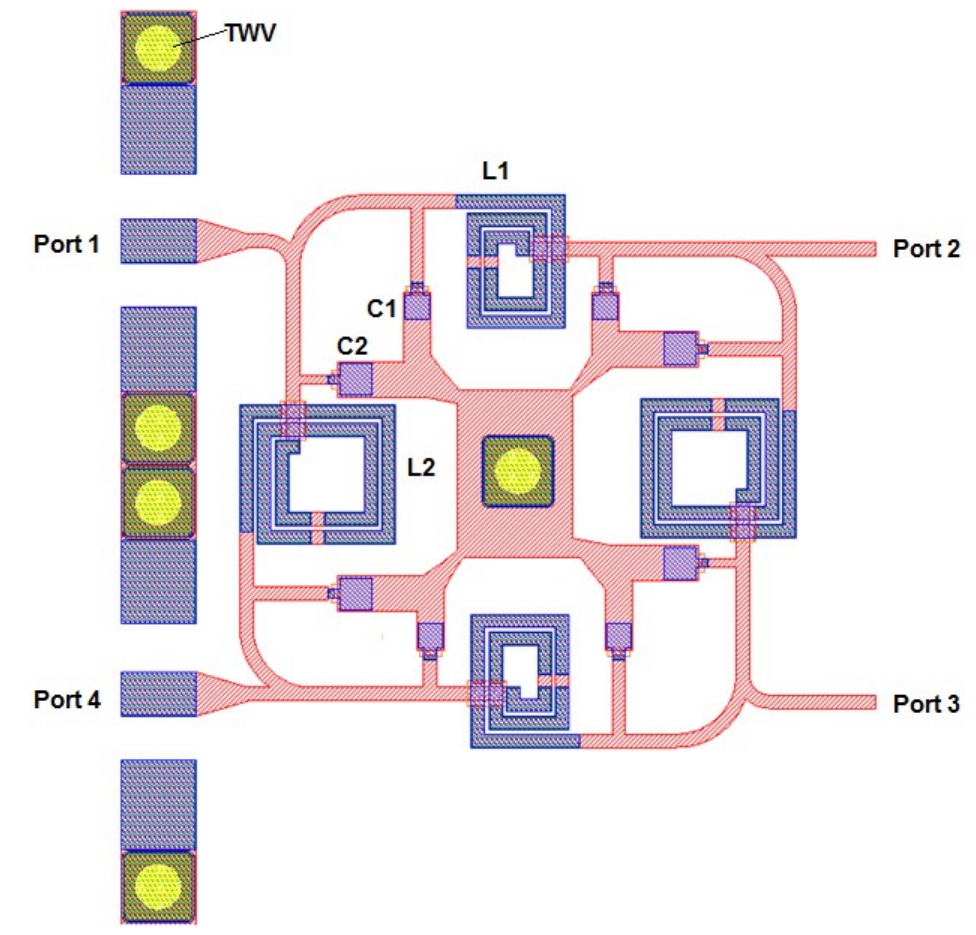

Figure IV.19: Layout of the designed hybrid coupler 


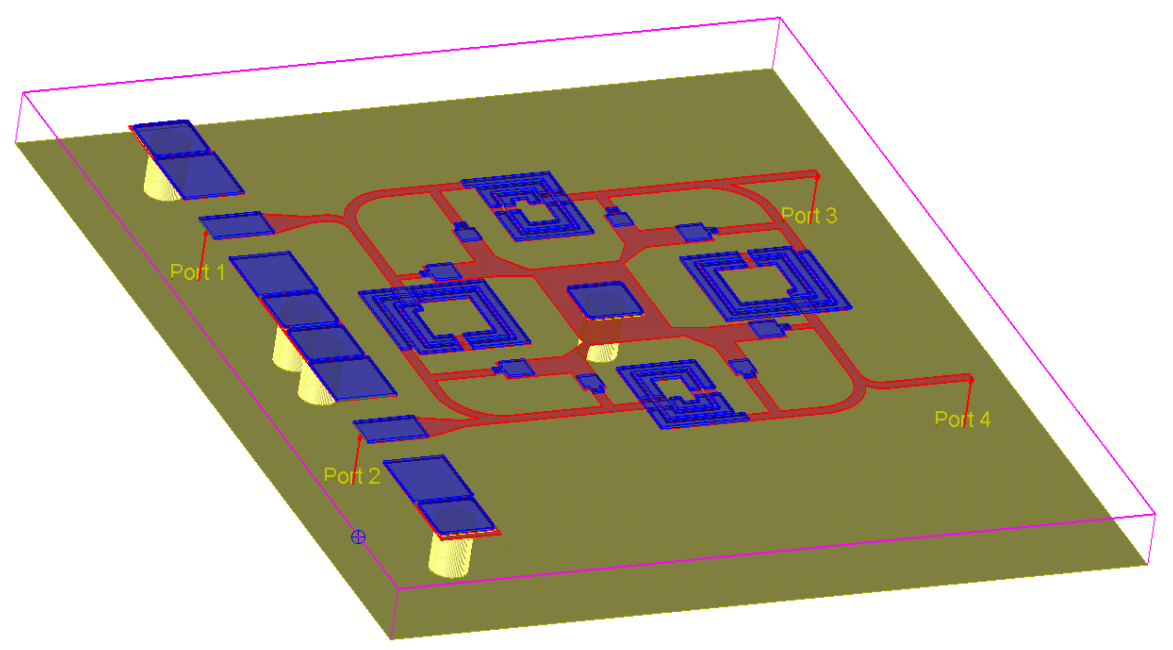

Figure IV.20: 3-D Layout view of the designed hybrid coupler

\subsubsection{Simulated Results and Discussion}

The ADS co-simulated layout results for the complete hybrid coupler are plotted in Figures from Figure IV.21 to Figure IV.24. The insertion loss at port 2 and port 3 are $-0.7 \mathrm{~dB}$ at $10 \mathrm{GHz}$ and has a maximum variation of $-0.4 \mathrm{~dB}$ over a $1 \mathrm{GHz}$ bandwidth (see Figure IV.21). The amplitude imbalance at the center frequency is almost $0 \mathrm{~dB}$. The phase difference (see Figure IV.24) between the outputs of ports 2 and 3 is about $89.5^{\circ}$ at $10 \mathrm{GHz}$ and varies by only $0.5^{\circ}$ over a $1 \mathrm{GHz}$ bandwidth. The return loss is better than $-20 \mathrm{~dB}$ at $10 \mathrm{GHz}$ (see Figure IV.22) and the isolation between the ports is better than -35dB (see Figure IV.23).

This compact $3-\mathrm{dB} 90^{\circ}$ GaN MMIC hybrid coupler based on lumped elements has been successfully designed by using NRC GaN150v1 process. The advantages of size reduction and good performance of the proposed $3-\mathrm{dB} 90^{\circ}$ hybrid coupler at X-band have been confirmed through the simulated results. Comparing to a reported GaAs hybrid coupler using distrubuted elements [106] with a size of $3.1 \times 2.3 \mathrm{~mm}^{2}$, this design (size of $0.85 \times 1 \mathrm{~mm}^{2}$ ) has shown a size reduction of $70 \%$ in the circuit area at $10 \mathrm{GHz}$. These results indicate that the proposed hybrid coupler is very promising for high density and tunable MMIC applications. Moreover, the hybrid coupler is used for designing a highly miniaturized and tunable phase shifter. 


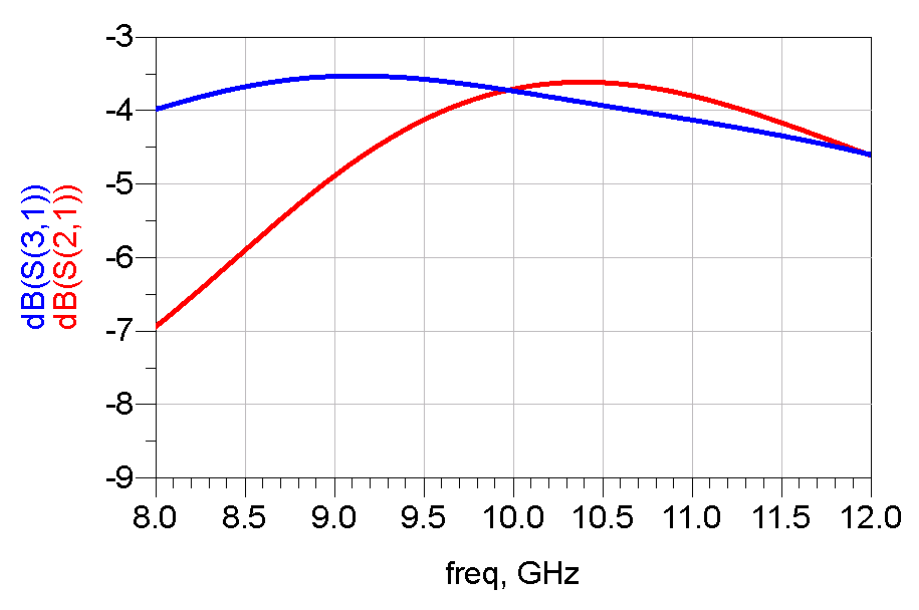

Figure IV.21: Insertion Loss versus frequency

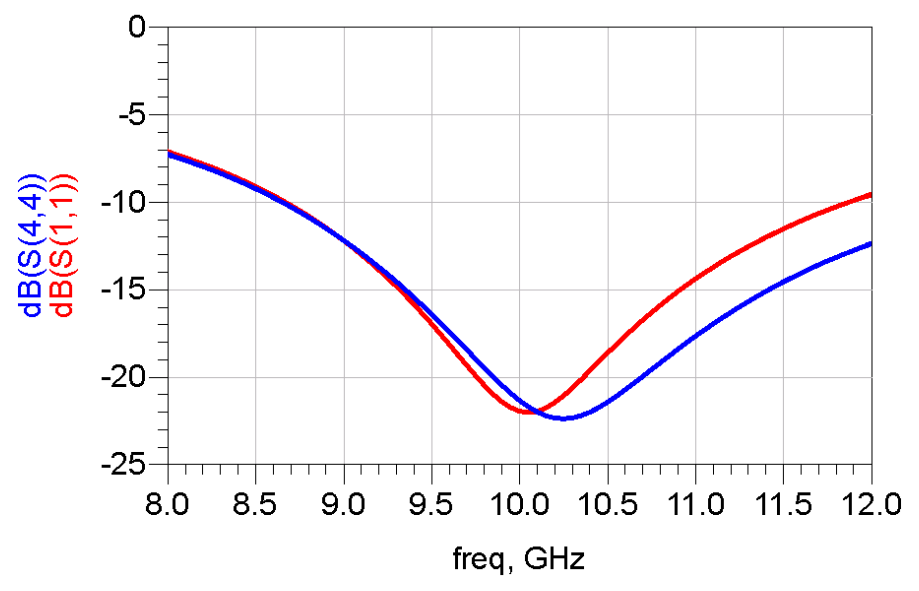

Figure IV.22: Return Loss versus frequency

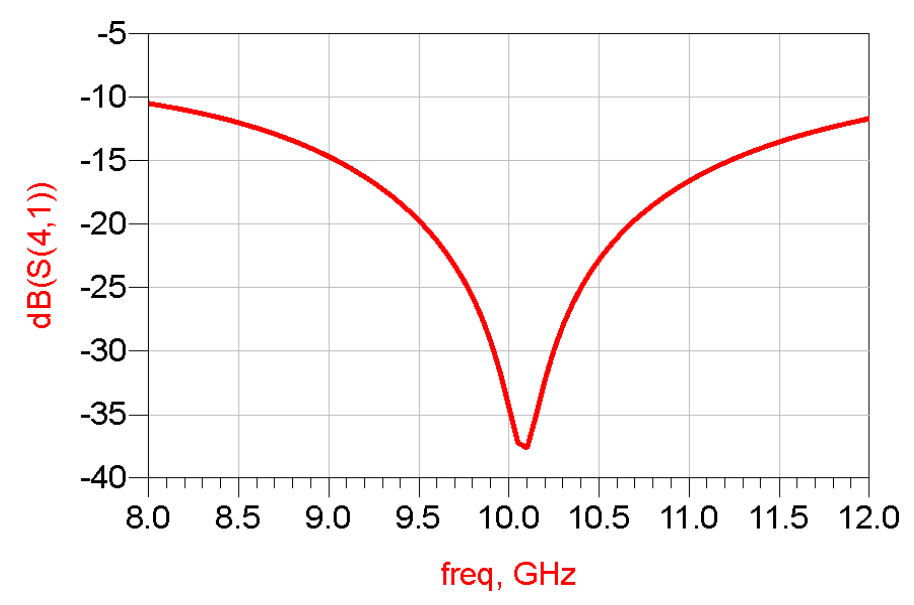

Figure IV.23: Isolation versus frequency 


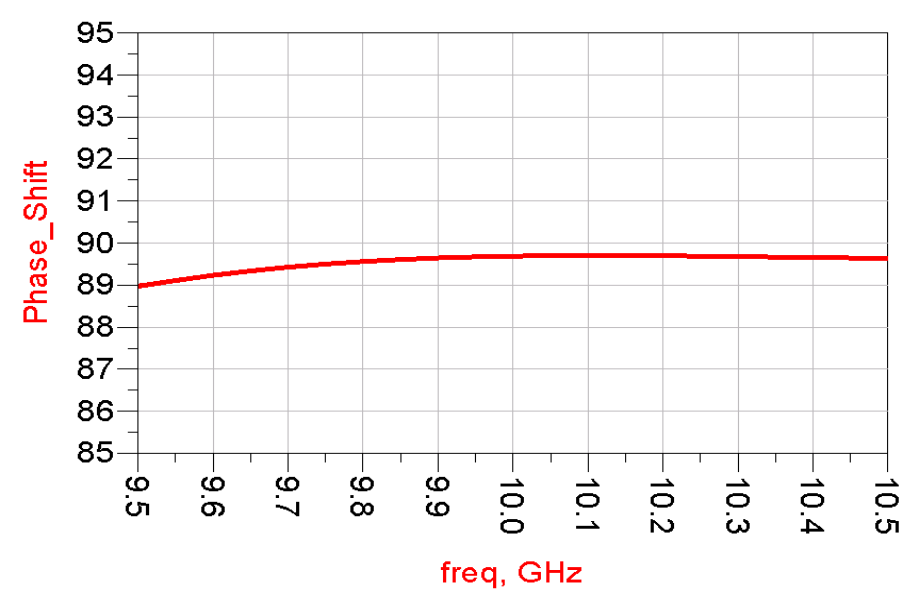

Figure IV.24: Phase Shift Difference versus frequency

\subsection{Phase Shifter Design}

As previously stated, the developed hybrid coupler will be used to design a phase shifter. This section discusses the design analysis of X-band GaN-based phase shifter. As depicted in Figure IV.25 and Figure IV.26, a layout photo as well as a 3-D view of the phase shifter are presented.

Here two identical GaN varactors were used as tuning loads purpose and placed at the transmitted and coupled ports of the hybrid coupler. These devices have a gate width of $200 \mu \mathrm{m}$ and two fingers for each. The actual used GaN device model is scaled from the two characterized original gate width GaN devices of 50um and $100 \mu \mathrm{m}$ model described previously in Chapter III. DC biasing is provided to the GaN varactors via DC probes with external bias tees. The circuit was simulated in Agilent's Advanced Design System (ADS) and Momentum was used to model the passive circuitry. The circuit was optimized to provide a maximum continuous phase shift at $10 \mathrm{GHz}$. 


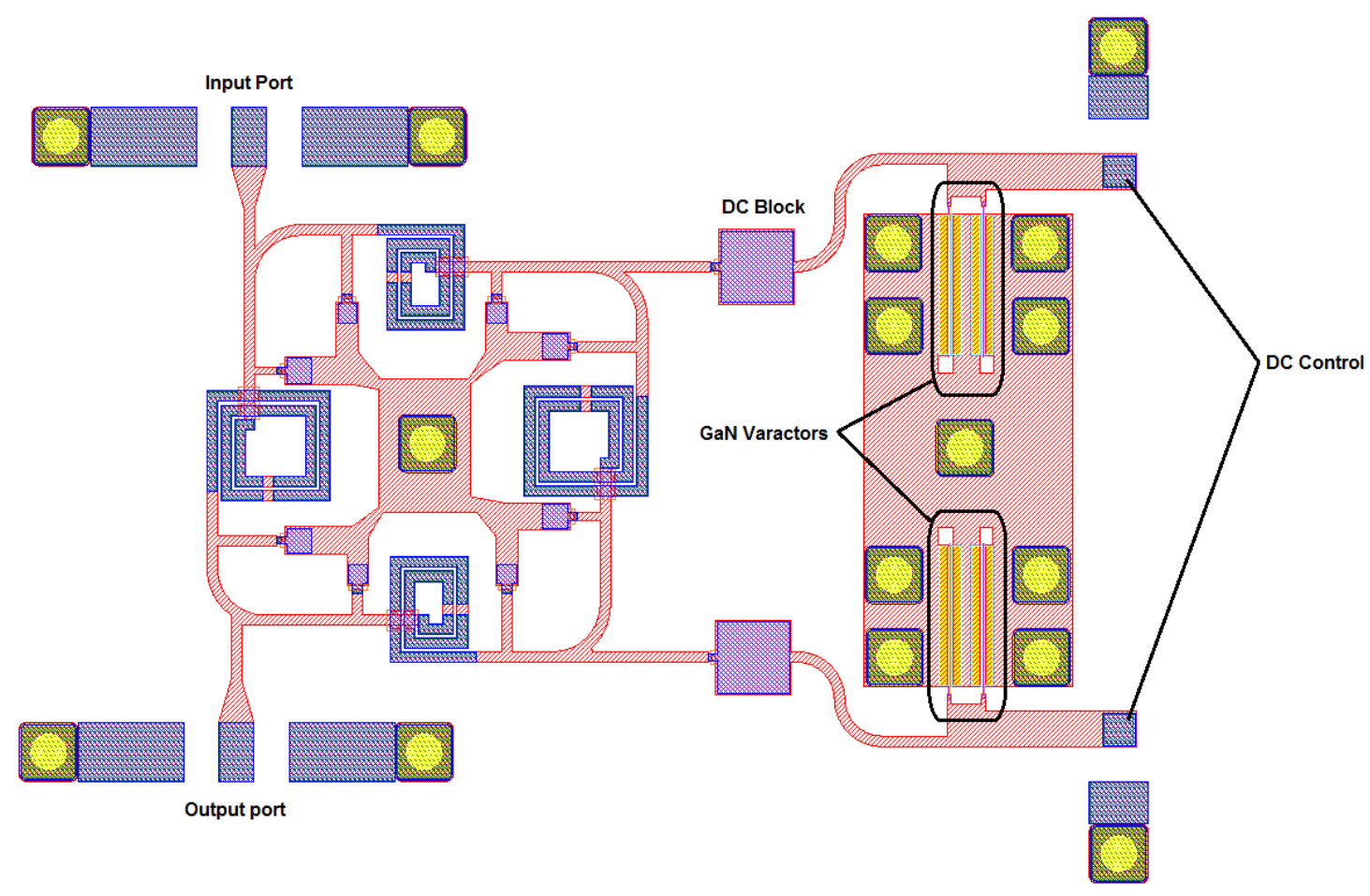

Figure IV.25: Layout of the designed Phase Shifter

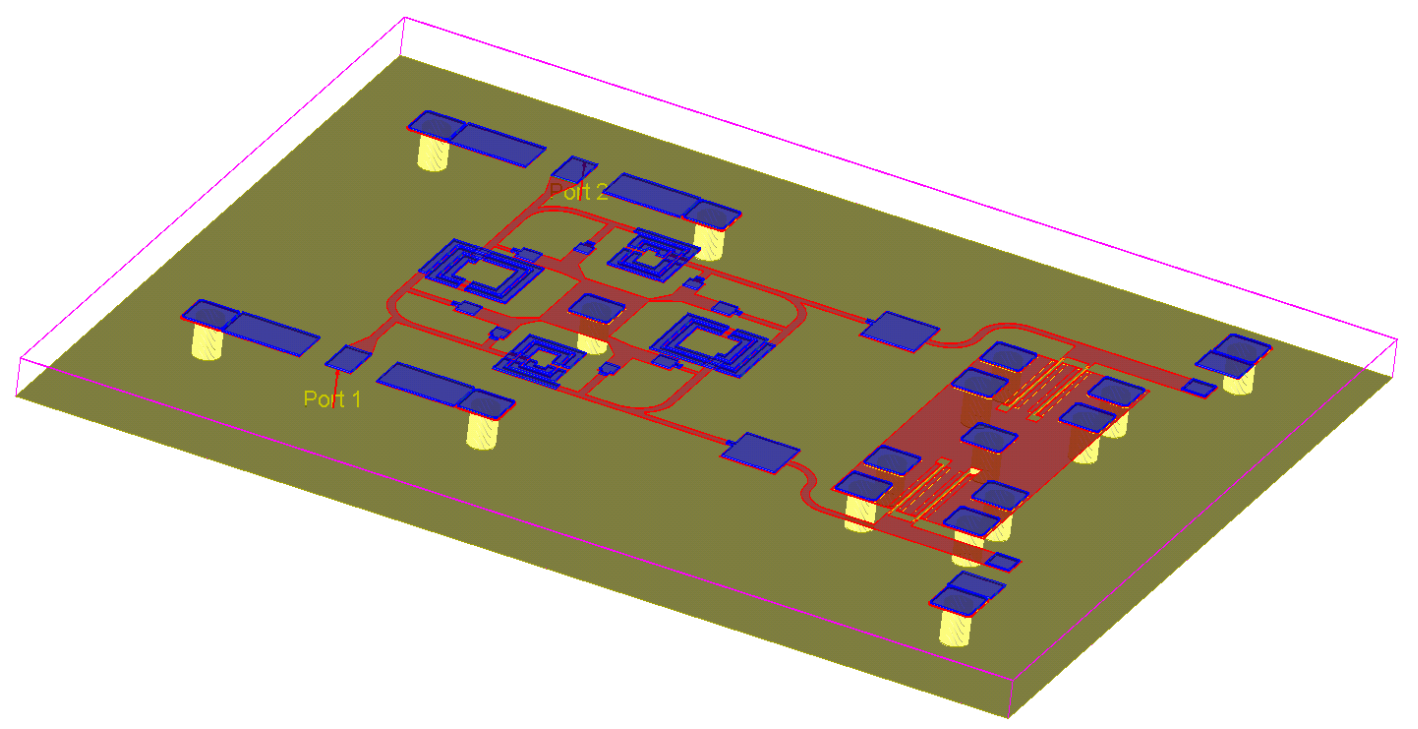

Figure IV.26: 3-D Layout view of the designed Phase Shifter 


\subsubsection{Simulated Results and Discussion}

In Figure IV.27, the return loss of the phase shifter under different bias conditions is presented and is better than $20 \mathrm{~dB}$ across a $1 \mathrm{GHz}$ bandwidth centered at $10 \mathrm{GHz}$. As clearly can be seen, a good match is achievable over this range of 9.510.5 $\mathrm{GHz}$ for all bias point varying from $-3.5 \mathrm{~V}$ to $-5.5 \mathrm{~V}$ which corresponding to the tuning range of the developed GaN varactor as stated previously in Chapter III.

Figure IV.28 shows the insertion loss of the phase shifter in different bias level from $-3.5 \mathrm{~V}$ to $-5.5 \mathrm{~V}$. At $10 \mathrm{GHz}$ worst case of the insertion loss is about $-3 \mathrm{~dB}$ and occurs at a bias point of $-4.3 \mathrm{~V}$. This value could be due to the higher series resistor value, $9 \Omega$, of the used varactor (i.e W100L0.15) at this bias voltage as reported in Chapter III (see Figure III.26). The amplitude imbalance between the two extreme DC bias states (i.e $-3.5 \mathrm{~V}$ and $-5.5 \mathrm{~V}$ ) is about $1.07 \mathrm{~dB}$ at $10 \mathrm{GHz}$, and is therefore better than $1.2 \mathrm{~dB}$ over the extended band from 9.5 to $10.5 \mathrm{GHz}$.

Figure IV.29 presents the phase shift obtained from the circuit versus DC bias at $10 \mathrm{GHz}$. Therefore, a continuously variable phase-shift range of $80^{\circ}$ is achieved as shown. This phase shift range versus DC bias exhibits the same general behavior as for equivalent capacitor of varactor versus DC bias as discussed in Chapter III in Figure III.24, which can be separated into three regions: range (1) for $\mathrm{V}_{\mathrm{G}}$ above $-3.5 \mathrm{~V}$ corresponding to the minimum phase shift value $\left(-80^{\circ}\right)$, range (3) for $\mathrm{V}_{\mathrm{G}}<-5 \mathrm{~V}$ corresponding to the maximum phase shift value $\left(0^{\circ}\right)$ and range (2) for $-5 \mathrm{~V}$ $\leq \mathrm{V}_{\mathrm{G}} \leq-3.5 \mathrm{~V}$ where the phase shift values change significantly and rapidly.

Maximum loss figure-of-merit (FOM) of this phase shifter of $74.76 \% \mathrm{~dB}$ at $10 \mathrm{GHz}$ and $66.66^{\circ} / \mathrm{dB}$ in the range of $9.5-10.5 \mathrm{GHz}$ were achieved. The loss FOM can be expressed as:

Equation (21)

$$
F O M=\frac{|\Delta \phi|^{\left({ }^{\circ}\right)}}{\alpha_{\max }{ }^{(d B)}}
$$


Where $\Delta \phi$ and $\alpha_{\max }$ are the differential phase shift and maximum insertion loss, respectively.

Based on this phase shifter structure, one varactor diode placed at the two coupler's output ports could enough to get a theoretical phase shift of $180^{\circ}$ as explained in [102]-[107]. However, varactor diodes are not perfect. This mean that there are losses due to their equivalent series resistor also these diode have a limited tuning capacitance range, thus makes it hard to achieve a $180^{\circ}$ complete phase shift.

The overall simulated performance and operating frequency range of this phase shifter are compared favorably with those of the reflection phase shifters using GaAs MMIC technology [108]-[109]-[110]. In [108] a phase shift of $109^{\circ}$ with an insertion loss of $1.8 \mathrm{~dB}$ over $16-18 \mathrm{GHz}$ was achieved by only placing one inductor and varactor at each coupler's output port. This inductance increases more the overall impedance (varactor and inductor) tuning range. It is possible to achieve a $360^{\circ}$ phase shifter range with $4.2 \mathrm{~dB}$ insertion loss in the same frequency range by connecting three phase-shifter chips in series as reported in this same work [108]. Using GaAs MMIC technology, another work [109] was presented where a phase shift of $210^{\circ}$ with a loss of $4.9 \mathrm{~dB}$ and a $1-\mathrm{dB}$ input compression point of higher than $5 \mathrm{dBm}$ was measured at $6.2 \mathrm{GHz}$. Based on the same structure, a miniaturized $\mathrm{Ku}$-band GaAs MMIC phase shifter using two quarter-wave-length transmission lines was reported in [110]. A phase shift of more than $180^{\circ}$ and an insertion loss of $3.6 \mathrm{~dB}$ are obtained at the frequency range from 12 to $14 \mathrm{GHz}$. The chip size of the experimental MMIC phase shifter is less than $3.0 \mathrm{~mm}^{2}$. 


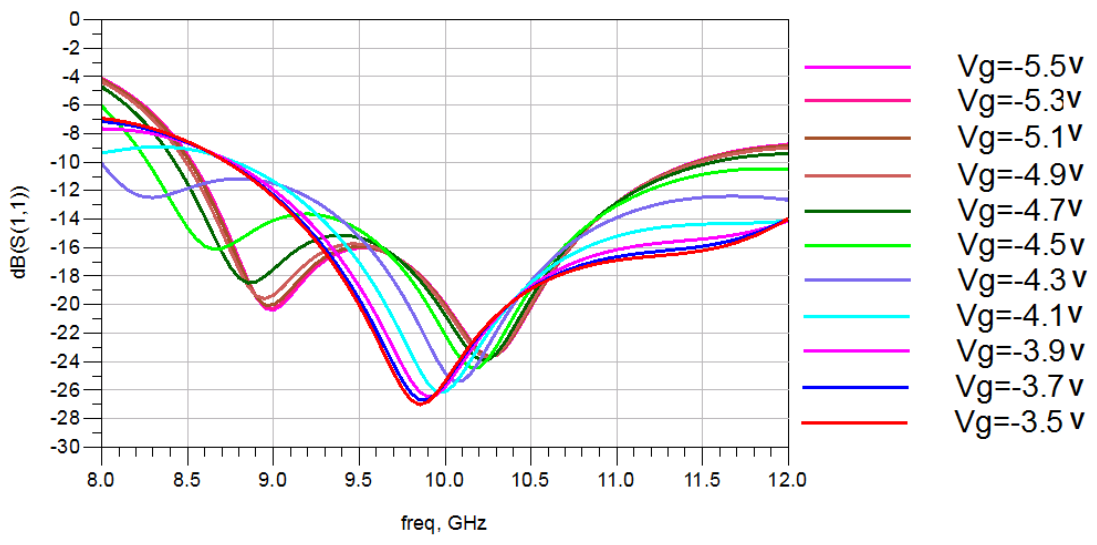

Figure IV.27: Return Loss at several DC Bias from $-3.5 \mathrm{~V}$ to $-5.5 \mathrm{~V}$

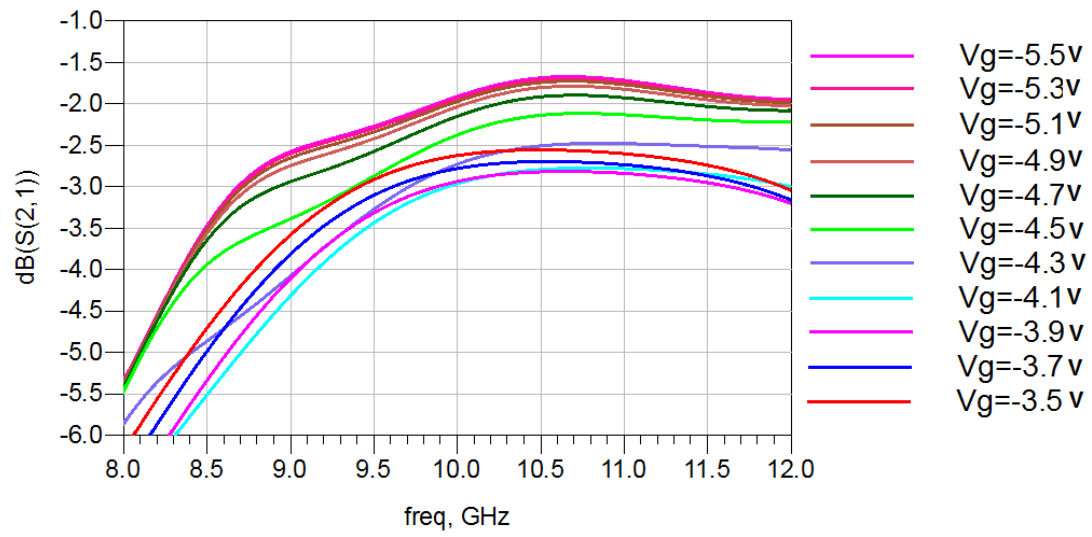

Figure IV.28: Insertion Loss of the Phase Shifter at several DC Bias from -3.5 V to $5.5 \mathrm{~V}$

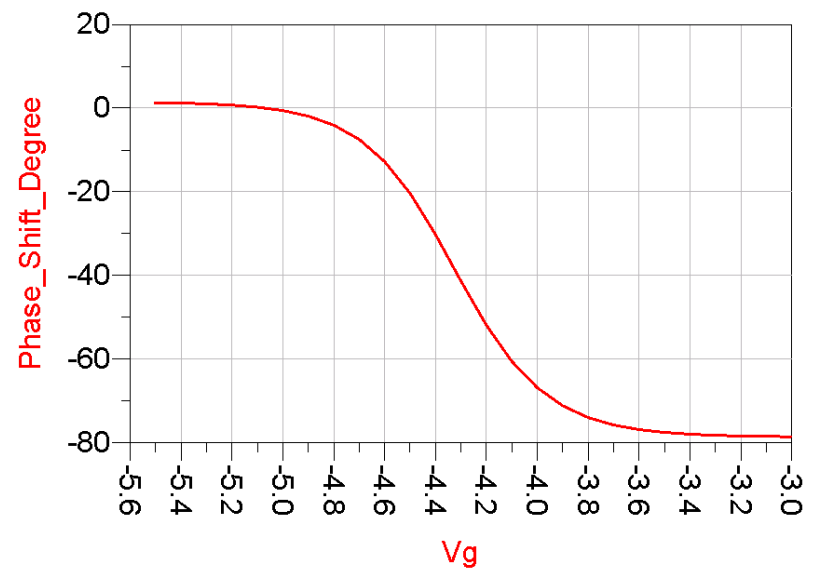

Figure IV.29: Phase Shift versus DC Bias at $10 \mathrm{GHz}$ 
In this section, a GaN phase shifter design based on reflective topology has been presented for high $\mathrm{RF}$ power source application. First, the $3-\mathrm{dB} 90^{\circ}$ hybrid coupler has been designed and simulated using NRC GaN150 PDK. Then two developed GaN varactors were paced on the two coupler's output ports as loads.

Under different bias conditions varying from $-3.5 \mathrm{~V}$ to $-5.5 \mathrm{~V}$, the return loss of the phase shifter is better than $20 \mathrm{~dB}$ across a $1 \mathrm{GHz}$ bandwidth centered at $10 \mathrm{GHz}$, while good match is achievable over this range of 9.5-10.5 GHz. Worst case

of the insertion loss is about $-3 \mathrm{~dB}$ at $10 \mathrm{GHz}$ and occurs at a bias point of $-4.3 \mathrm{~V}$ where the equivalent resistor of the used varactors is significantly high. Therefore, a continuously variable phase-shift range of $80^{\circ}$ is achieved at $10 \mathrm{GHz}$. These achieved performances were compared favorably with other works that use GaAs technology.

\section{GaN Reconfigurable 3-dB/90 Hybrid Coupler}

\section{Review}

Based on the previous presented $10 \mathrm{GHz}$ GaN hybrid coupler, a frequency reconfigurable $3-\mathrm{dB} 90^{\circ}$ hybrid coupler is designed. In order to achieve frequency reconfigurability, varactors diodes are used instead of the four fixed shunt lumped capacitors $\mathrm{C}_{1}$ of the previous static frequency hybrid coupler (see Figure IV.19). In this configuration, an electronically frequency tuned is achieved by changing the bias voltage applied across the varactors. This means that changing the resonance frequency of branch-line (LC parallel resonator) hybrid of the coupler, we can provide the different operating frequency bands. Additionally, the tuned frequency of the coupler is defined as the frequency at which high isolation and low return loss are achieved, while realizing phase shift of $90^{\circ}$ between the isolated and the coupled ports. The novel tunable GaN hybrid coupler employs the developed GaN varactors elements to provide the reconfigurability from $10 \mathrm{GHz}$ to $16 \mathrm{GHz}$. 
Recently, considerable work in tunable couplers consist of MMIC-based active couplers and varactor diode based passive couplers have been reported in the literature which most of them are operating below X-band frequency. However, most of these reported couplers exhibit, in general, a small frequency tuning range. It is still a challenging task to design tunable couplers with tuning ranges wide enough to cover desired frequency bands. For instance, in [111] a tunable hydrid coupler is reported using GaAs technology. This presented tunable GaAs MMIC directional coupler design uses tunable capacitances to achieve a tunable coupling coefficient $(|\mathrm{S} 31|)$ at $2 \mathrm{GHz}$. Over a bias range of $0 \mathrm{~V}$ to $-1.5 \mathrm{~V}$ the measured S-parameters for the coupled port demonstrate a tuning range of $-6.6 \mathrm{~dB}$ to $-60 \mathrm{~dB}$. The input return loss is better than $-12 \mathrm{~dB}$ for all bias voltage levels with isolation better than $-17 \mathrm{~dB}$. This design is implemented in a $0.5 \mu \mathrm{m}$ GaAs process with Schottky varactor diodes, and occupies an area of $2.2 \times 1.4 \mathrm{~mm}^{2}$. In [112] a frequency tunable broadband MMIC active directional coupler is discussed. The coupler provides various coupling gains such as $3 \mathrm{~dB}, 0 \mathrm{~dB},-3 \mathrm{~dB}$, and $-6 \mathrm{~dB}$, also a tuned frequency from $5.5 \mathrm{GHz}$ to $7.8 \mathrm{GHz}$ was achieved by applying different bias voltages. Using varactors for tunability purpose, a novel compact dual-band reconfigurable power divider is presented in [113] for smart antenna applications. Measured results show that, by biasing one varactor and keeping the other one at $0 \mathrm{~V}$, good performance are achieved at the center frequency $2.45 \mathrm{GHz}$, while this frequency can be tuned to $5.25 \mathrm{GHz}$ by only switching the bias state of varactors. Also, any value of power ratio can be selected from 1:0 to 0:1 for both the center frequencies $2.45 \mathrm{GHz}$ and $5.25 \mathrm{GHz}$, maintaining an average insertion loss better than $-1.5 \mathrm{~dB}$ and $-3 \mathrm{~dB}$ in the $2.45 \mathrm{GHz}$ and $5.25 \mathrm{GHz}$ bands, respectively, along with a return loss better than $-15 \mathrm{~dB}$ in both frequency ranges. Measured isolation at port 2 is always better than $-20 \mathrm{~dB}$ in both frequencies. 


\section{Design of Reconfigurable $3-\mathrm{dB} / 90^{\circ}$ Coupler}

In this context, here we report a continuous broadband frequency tunable GaN hybrid coupler. The achieved tuning range is from $10 \mathrm{GHz}$ to $16 \mathrm{GHz}$ corresponding to a bandwidth of $46 \%$. As far as the authors know, this proposed frequency reconfigurable hybrid coupler is the first reported work that uses GaN HEMTs technology. Figure IV.30 and Figure IV.31 show the tunable layout design version of the coupler, where the shunt MIM capacitors are replaced by the developed GaN varactors model. The varactors provide the function of frequency tunability by adjusting their voltage level. This varactor device, W200L0.15, is a two fingers and 200 um gate length and is fabricated using GaN150 process.

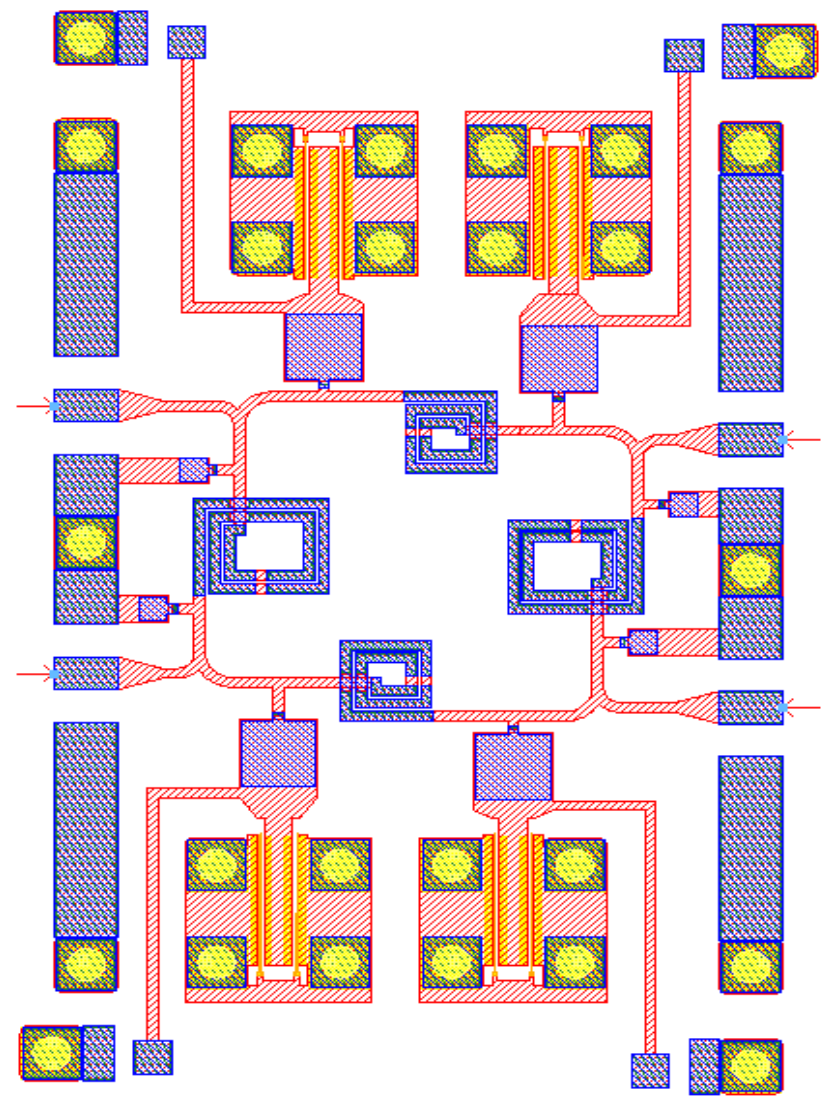

Figure IV.30: Layout of the designed hybrid coupler 


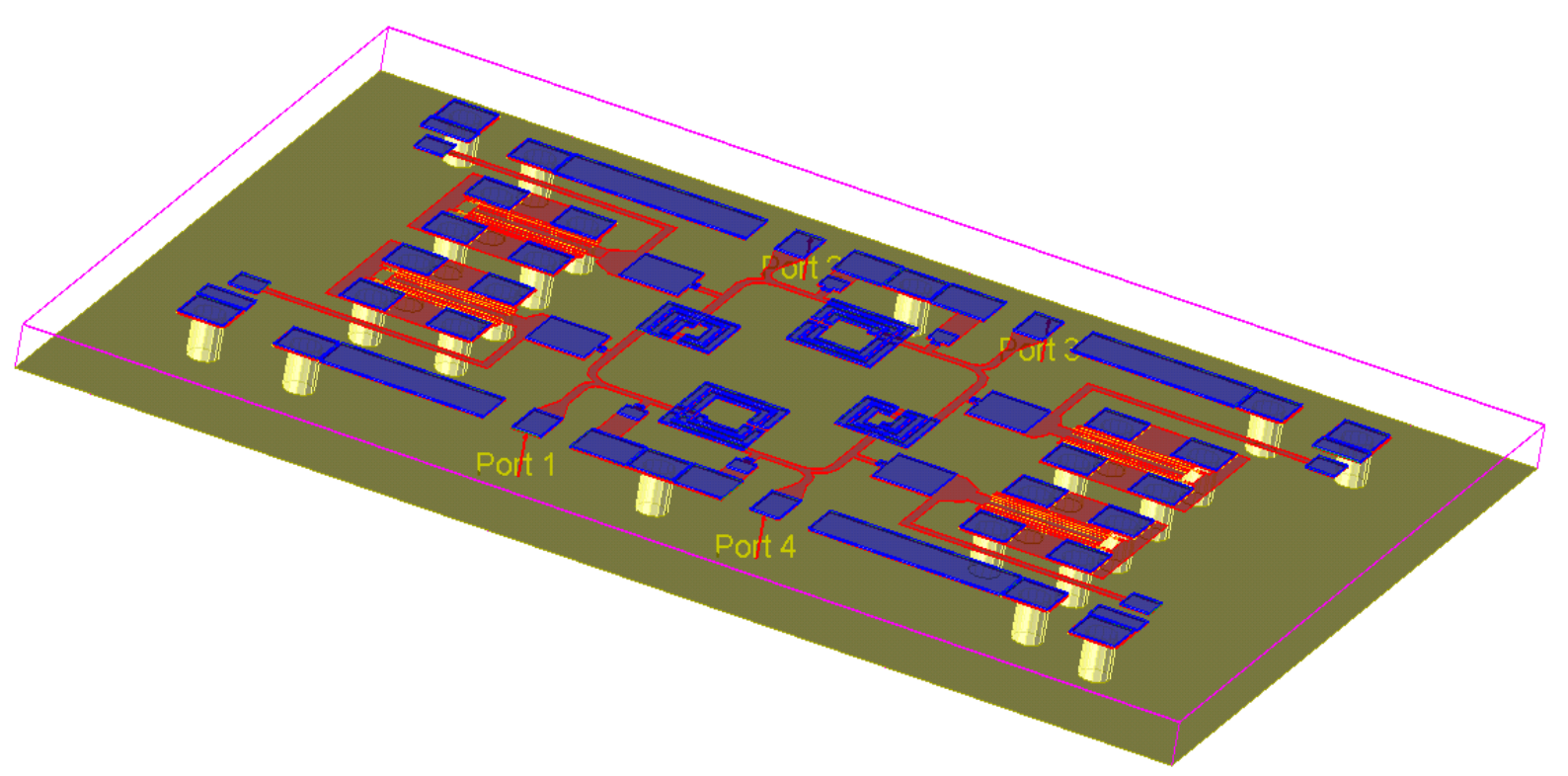

Figure IV.31: 3-D Layout view of the designed hybrid coupler

\subsection{Simulated Results and Discussion}

Simulation results, which are shown in Figure IV.32, Figure IV.33, Figure IV.34, Figure IV.35 and Figure IV.36, respectively, are illustrating, at several DC level voltage for return loss, isolation, power division at transmitted port and loss insertion at coupled port.

The overall return loss depicted in Figure IV.32 is better than $-13.5 \mathrm{~dB}$ over all the tuning range from $-3.7 \mathrm{~V}$ to $-4.8 \mathrm{~V}$. This worst case of $-13.5 \mathrm{~dB}$ is achieved at a $\mathrm{DC}$ bias of $-3.7 \mathrm{~V}$ corresponding to the maximum value of varactor capacitance. In Figure IV.33, the isolation is clearly below than $-14 \mathrm{~dB}$ over the full tuning range.

The simulated magnitude of S21 parameter, as illustrated in Figure IV.34, is close to $-3.5 \mathrm{~dB}$ and is getting poorer as varactor capacitance values decreases. $\mathrm{A}$ worst value of $-5 \mathrm{~dB}$ is achieved at the minimum capacitance value. While at port 3 , the S31 parameter (see Figure IV.35) behaves as S21 but in a reverse way. This means the worst case which is about $-3.7 \mathrm{~dB}$ achieved when the varactor capacitance 
is at its maximum. As a result, the amplitude imbalance is varying from $-0.2 \mathrm{~dB}$ at $10 \mathrm{GHz}$ and $-2 \mathrm{~dB}$ at $16 \mathrm{GHz}$.

A minimum well-balanced quadrature phase of $86.5^{\circ}$ is obtained between the coupled port 2 and the through port 3 as plotted in Figure IV.36. This phase is increasing and getting closer to $90^{\circ}$ as varactor capacitor values are decreasing. The tunable quadrature coupler has $46 \%$ center-frequency tunable range from approximately 10 to $16 \mathrm{GHz}$.

There are four DC blocking capacitors on each port to block the DC bias from the output. This simplified bias network aids in reducing the overall size and complexity of the circuit.

Unfortunately, as for the phase shifter, this design has not been manufactured because the run of manufacturing on GaN150 process has not been done in time by NRC.

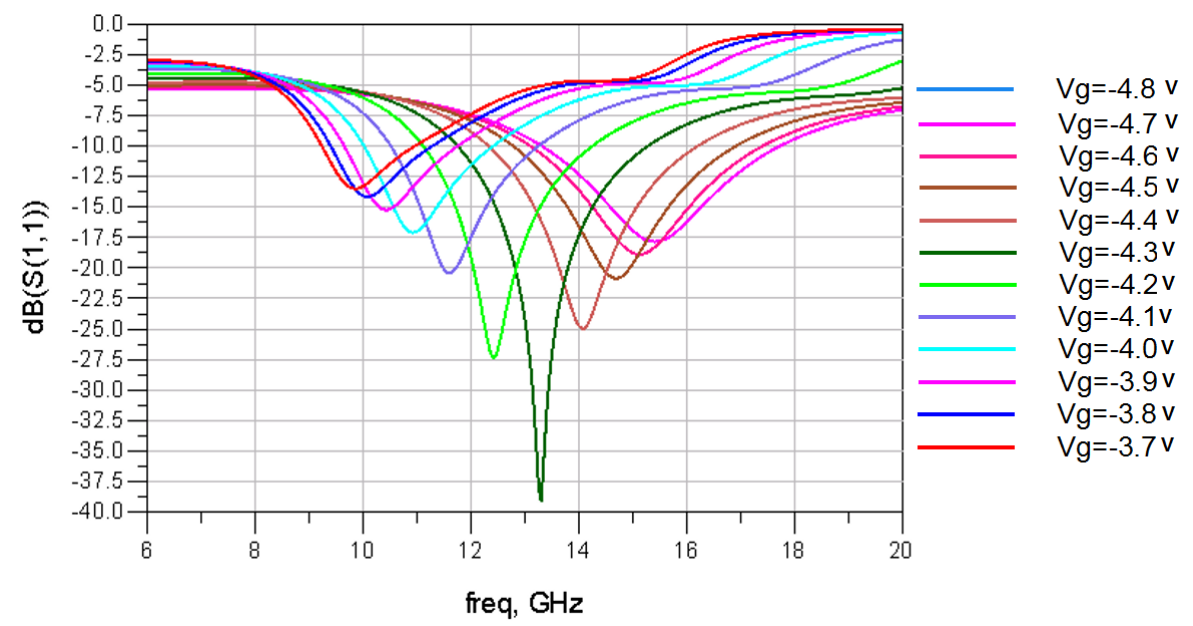

Figure IV.32: Return Loss at several DC Bias from $-3.7 \mathrm{~V}$ to $-4.8 \mathrm{~V}$ 


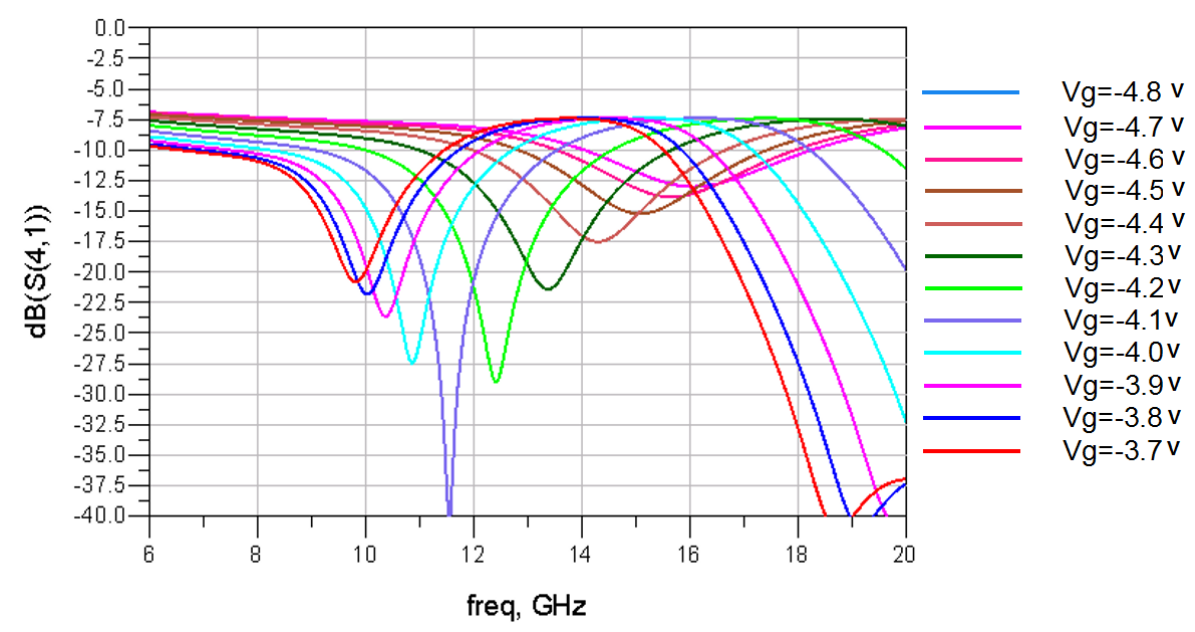

Figure IV.33: Isolation at several DC Bias from $-3.7 \mathrm{~V}$ to $-4.8 \mathrm{~V}$

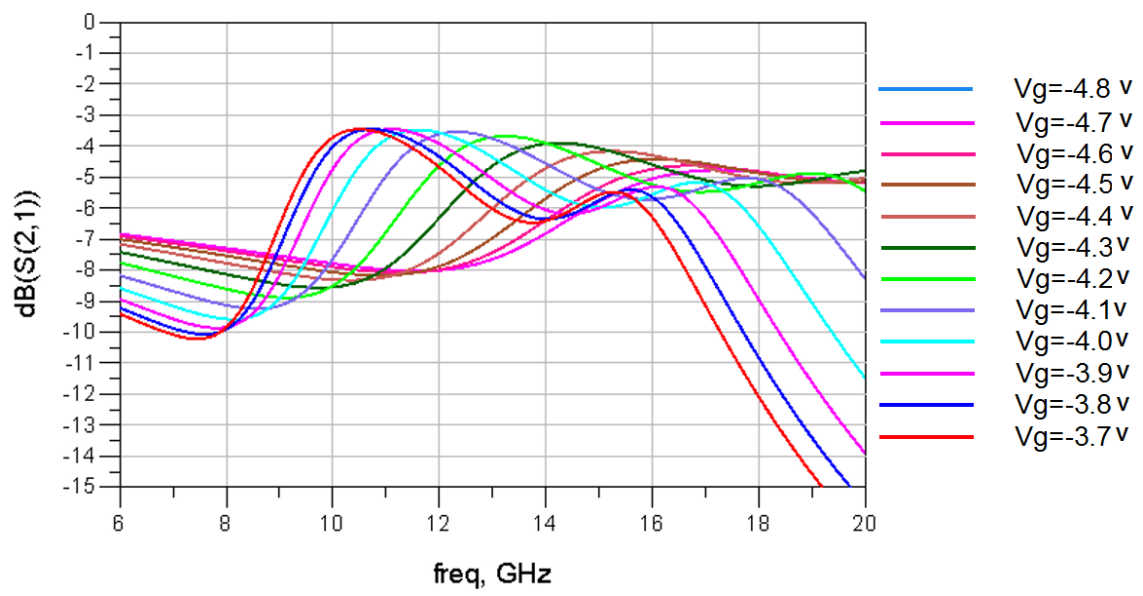

Figure IV.34: Level of S21 at several DC Bias from $-3.7 \mathrm{~V}$ to $-4.8 \mathrm{~V}$

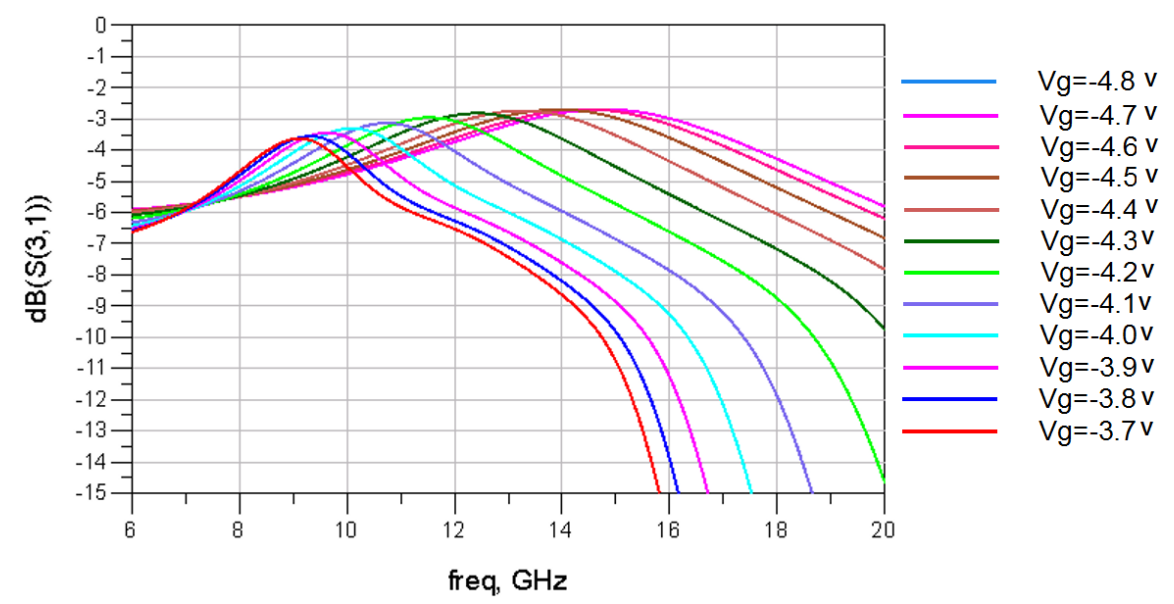

Figure IV.35: Level of S31 at several DC Bias from $-3.7 \mathrm{~V}$ to $-4.8 \mathrm{~V}$ 


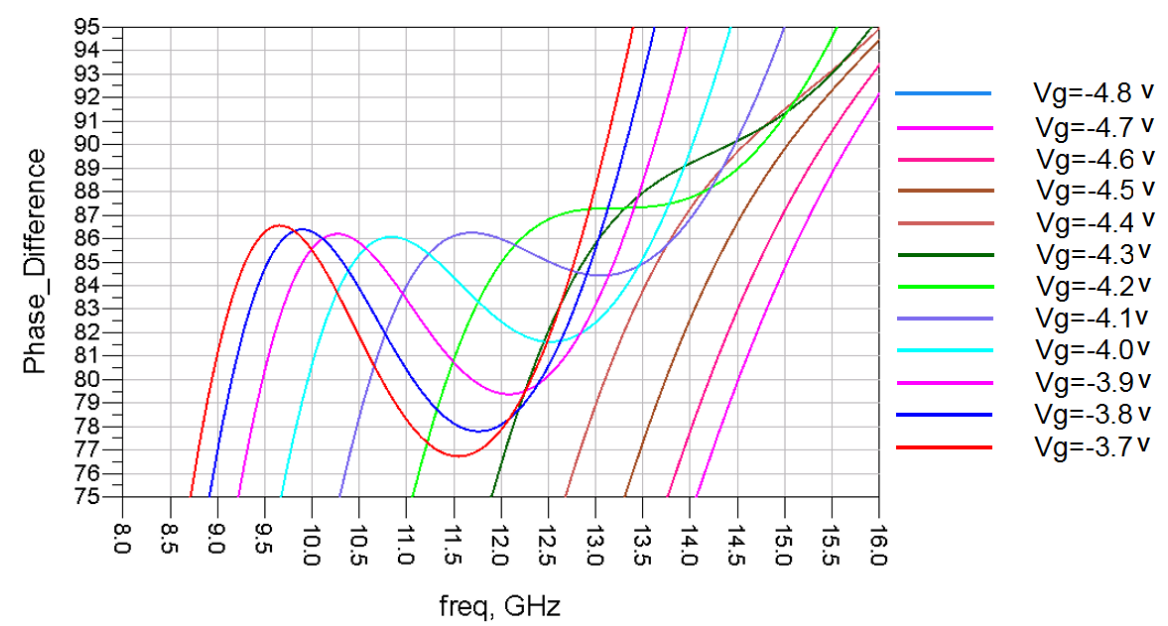

Figure IV.36: Phase Difference at several DC Bias from $-3.7 \mathrm{~V}$ to $-4.8 \mathrm{~V}$

A varactor tunable broadband quadrature hybrid coupler has been investigated and simulated from $\mathrm{X}$-band to $\mathrm{Ku}$-band frequencies applications. First, a static coupler has been designed to meet the specifications of a typical quadrature hybrid coupler (3- $\mathrm{dB}$ insertion loss, good return and isolation loss, and $90^{\circ}$ phase difference between coupled and through ports) at $10 \mathrm{GHz}$. Then, a tunable broadband coupler has been designed by inserting varactor diodes as shunt capacitance elements in order to control the operating frequency. This design is implemented in a $0.15 \mu \mathrm{m}$ GaN process from NRC, and occupies an area of $1.35 \times 0.9 \mathrm{~mm}^{2}$. Good simulation results have been demonstrated.

This novel varactor reconfigurable hybrid coupler is proposed to overcome the need of high power tunable $\mathrm{RF} /$ microwave circuits which are required for multistandard and multiservice applications. By changing the bias voltage of the varactor to control the resonance condition of parallel LC resonator, this proposed hybrid coupler can provide the desired operating frequency from $10 \mathrm{GHz}$ to $16 \mathrm{GHz}$. 


\section{GaN-based Oscillator}

\section{Review}

Microwave oscillators are widely used in wireless communication systems. An oscillator is an electronic circuit which can generate a periodic signal by converting DC power to RF power. Two approaches, such as feedback oscillator and negative resistance oscillator [114], are commonly used for the design of oscillators. In the feedback topology, the oscillator is constituted of an amplifier with a positive feedback network (see Figure IV.37) that are forming a loop. The condition for oscillation is that a part of output signal is combined in phase with the input signal and the amplitude of the loop gain is larger than unity. This topology is used to design two oscillators in this thesis.

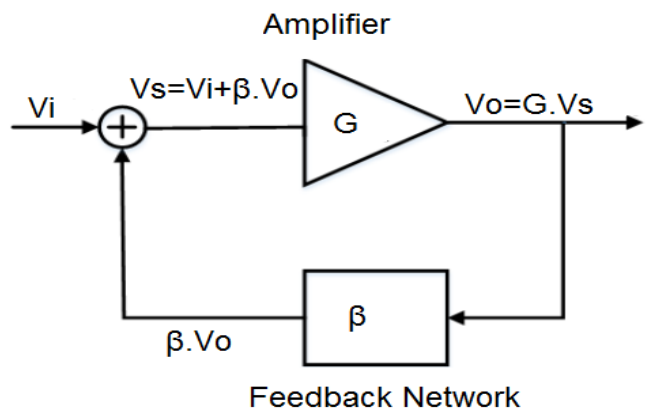

Figure IV.37: Block diagram of a feedback oscillator

In state-of-the art most of low-phase noise oscillators are designed in mature semiconductor technology such as GaAs, InP and SiGe, with key property for good low phase noise. However, one of the drawbacks of such oscillators is that the output power is limited by the relatively low breakdown voltage. In the other hand, GaN HEMTs technology with significantly higher breakdown voltage is become more and more used in oscillators that potentially reach relatively low phase noise [115], very high power handling capabilities and good linearity. GaN oscillators also have the advantage of not needing an additional output buffer amplifier stage between 
them and a load, thus simplifying the circuit. As a result, a GaN-based system will be less complex, and thus smaller.

Currently, a number of published works have been devoted to improve the high power performances of oscillators by adopting GaN HEMTs technology with high power capabilities advantages. Most of these reported GaN-based oscillators are designed mainly for low frequency such L-band [116]-[117], S-band [118]-[119]- [120], Cband [121]-[122]-[123]-[124]-[125]-[126]-[127]-[128] and X-band [129]-[130]- [131]- [132][133]- [134], while there are a few reported works for millimeter-wave frequency such as Q-band [135] and V-band [136].

Earlier in 2001, the first report of AlGaN/GaN HEMT-based VCOs operating at $6 \mathrm{GHz}$ is presented in [121]. An output power of $27 \mathrm{dBm}$ and phase noise level of $-92 \mathrm{dBc} / \mathrm{Hz}$ at $100 \mathrm{KHz}$ offset were achieved. This achieved phase noise value was favorably equivalent to the single-sideband noise of a GaAs FET-based oscillator [137]. It is also clearly shown in [121] that GaN oscillators with large tuning range can achieve a phase noise of $-123 \mathrm{dBc} / \mathrm{Hz}$ at $1 \mathrm{MHz}$, whereas those fixed frequency ones can achieve a phase noise as low as $-135 \mathrm{dBc} / \mathrm{Hz}$ [125] at $1 \mathrm{MHz}$. To the authors' knowledge of [125], this GaN-based oscillator achieves the lowest phase noise compared to other GaN HEMT based integrated oscillators. An output power of 21 $\mathrm{dBm}$ is achieved at $7.9 \mathrm{GHz}$ when the device is biased at Vgs of $-3 \mathrm{~V}$ and $\mathrm{Vds}$ of $28 \mathrm{~V}$. Another design is presented in [122], which used an in-house GaN HEMT facility. The phase noise of the designed $5 \mathrm{GHz}$ oscillator can be kept more or less as low, -105 $\mathrm{dBc} / \mathrm{Hz}$ at $100 \mathrm{kHz}$, as the ones $(-112 \mathrm{dBc} / \mathrm{Hz}$ at $100 \mathrm{kHz})$ designed in GaAs [138] while an output power of more than $20 \mathrm{dBm}$ is achieved. Still over the C-band, differential GaN oscillators are reported in [123]. An output power level of $23 \mathrm{dBm}$ at $4.16 \mathrm{GHz}$ is reported, while phase noise is $-86 \mathrm{dBc} / \mathrm{Hz}$ at offsets of $100 \mathrm{kHz}$ and $-115.7 \mathrm{dBc} / \mathrm{Hz}$ at offsets of $1 \mathrm{MHz}$. The second and third harmonic suppression are equal to $-45 \mathrm{~dB}$ and -70dB respectively. High power single-ended Colpitts-type GaN oscillators using field-plated HEMTs are described in [126]. Both output power and operation bias can 
be significantly increased to $32.8 \mathrm{dBm}$ and $V d s=40 \mathrm{~V}$ with an output power density of $3.8 \mathrm{~W} / \mathrm{mm}$ at $5 \mathrm{GHz}$. The DC-to-RF-conversion efficiency amounts to $21 \%$, while the measured phase noise at $1 \mathrm{MHz}$ offset from the carrier amounts to $-132 \mathrm{dBc} / \mathrm{Hz}$. A recent C-band work is reported in [128] where a MMIC GaN HEMT VCO with high tuning linearity and low phase noise is presented. The designed VCO is based on a balanced Colpitts topology and is tunable between $6.45-7.55 \mathrm{GHz}$ with good tuning linearity, constant output power of $2 \mathrm{dBm}$ at a bias condition of $\mathrm{Vd}=6 \mathrm{~V}$ and an associated Id of $33 \mathrm{~mA}$, and a good phase noise with little variation over the tuning range. The measured phase noise is $-98 \mathrm{dBc} / \mathrm{Hz}$ at $100 \mathrm{kHz}$ and $-132 \mathrm{dBc} / \mathrm{Hz}$ at $1 \mathrm{MHz}$ offset frequency.

Over the X-band frequency, some GaN-based oscillators have been implemented. In [129] a VCO with an output power of $35 \mathrm{dBm}$ between 8.5 and $9.5 \mathrm{GHz}$ is presented. Output power density of $2.1 \mathrm{~W} / \mathrm{mm}$ is reached at $V d s=30 \mathrm{~V}$. The technology uses GaN HEMTs with a gate length of $0.15 \mu \mathrm{m}$. The phase noise is estimated to be $-87 \mathrm{dBc} / \mathrm{Hz}$ at $100 \mathrm{kHz}$ offset. Another interesting X-band GaN HEMTs-based oscillator is also designed in an in-house facility and is presented in [130]. The VCO exhibits a frequency tuning range from 9.11 to $9.55 \mathrm{GHz}$, an average output power of $3.3 \mathrm{dBm}$ with phase noise of $-82 \mathrm{dBc} / \mathrm{Hz}$ at $100 \mathrm{kHz}$ offsets and -110 $\mathrm{dBc} / \mathrm{Hz}$ at $1 \mathrm{MHz}$. These performances are investigated under a $5 \mathrm{~V}$ drain bias instead of utilizing large device with high drain bias to achieve the high output power. Although the phase noise of this oscillator still needs to be further improved, it opens up a new examination on the design of oscillators in GaN HEMT technology for low voltage, moderate output power as well as low phase noise. A design of an X-band GaN oscillator is discussed in [131]. At $9.9 \mathrm{GHz}$, an output power of $20 \mathrm{dBm}$ is measured, with a phase noise level around $-105 \mathrm{dBc} / \mathrm{Hz}$ at $100 \mathrm{kHz}$ offset frequency, but with a poor $2 \mathrm{nd}$ harmonic found at $-10 \mathrm{dBc}$, while the third harmonic is rejected more than $-30 \mathrm{dBc}$ from the carrier. An X-band oscillator is presented in [132] using common-gate balanced Colpitts oscillator topology. A commercial Triquint's $0.25 \mu \mathrm{m}$ GaN MMIC HEMT process is used. Using the same commercial GaN HEMT process 
as in [132], a recent X-band ultra-low phase-noise GaN HEMT oscillator is described in [134] by the same authors of [132]. The designed oscillator is based on a GaN HEMT MMIC reflection amplifier and a rectangular aluminum cavity. It oscillates at 9.9 $\mathrm{GHz}$ with an output power of $5 \mathrm{dBm}$ and an excellent phase noise of $-145 \mathrm{dBc} / \mathrm{Hz}$ at $100 \mathrm{kHz}$ and $-160 \mathrm{dBc} / \mathrm{Hz}$ at $400 \mathrm{kHz}$. These are the best reported phase noise values so far by using GaN HEMT devices. Going higher in frequency, a low phase noise power-efficient MMIC GaN-HEMT Oscillator at $15 \mathrm{GHz}$ based on a Quasi-lumped onchip resonator is reported in [133]. An excellent phase noise of -106 dBc/Hz and $133 \mathrm{dBc} / \mathrm{Hz}$, respectively, at $100 \mathrm{kHz}$ and $1 \mathrm{MHz}$ offsets and a low output power of -6 $\mathrm{dBm}$ are experimentally demonstrated.

A Q-band MMIC oscillator (39GHz) is presented in [133]. An output power of $25 \mathrm{dBm}$ at $V d s=25 \mathrm{~V}(\mathrm{Id}=156 \mathrm{~mA})$ is reported with a low-phase noise level of -120 $\mathrm{dBc}$ at $1 \mathrm{MHz}$ offset from the carrier. The $\mathrm{DC}-$ to RF-conversion efficiency is about $12 \%$. Around $53 \mathrm{GHz}$, the same authors report a new V-band GaN MMIC VCO [136] realized on a gate length of $0.2 \mu \mathrm{m} \mathrm{AlGaN/GaN} \mathrm{HEMT} \mathrm{process.} \mathrm{The} \mathrm{delivered} \mathrm{output}$ power is around $11 \mathrm{dBm}$ at $53 \mathrm{GHz}$ with an estimated phase noise of $-97 \mathrm{dBc} / \mathrm{Hz}$ at 1 $\mathrm{MHz}$ offset with bias conditions of $\mathrm{Vd}=15 \mathrm{~V}$ and $\mathrm{Vg}=-3 \mathrm{~V}$. These reported works [135]-[136] demonstrate the potential of using GaN technology for military and commercial applications that require high frequency, high power, and low phase noise frequency sources.

\section{Design of GaN-based Oscillators}

In this perspective, using GaN HEMT technology, two X-band oscillators are presented in this section: a fixed frequency oscillator (FFO) and a tunable frequency oscillator (TFO). The two oscillators are designed based on feedback oscillator topology and are fabricated. The GaN HEMT based FFO is designed to operate around $10 \mathrm{GHz}$ while the GaN HEMT based TFO is tunable between 7.8-8.6 $\mathrm{GHz}$, corresponding to a tuning range close to $10 \%$. The frequency reconfigurability 
of the designed TFO oscillator is based on the in-house developed GaN varactor detailed in chapter III.

\subsection{Feedback Oscillator Topology}

Oscillator based on feedback topology using amplifier approach is introduced in this section. The GaN PA design used here is presented in next section. A frequency-selective feedback network printed on Neltec NX9300 substrate is designed to return part of the output signal to the amplifier input. From Figure IV.37, it can quickly derive the expression of the transfer function of the feedback oscillator as:

Equation (22)

$$
\frac{V o}{V i}=\frac{G}{1-\beta \cdot G}
$$

This design topology offers some very attractive features, including a straightforward design, a relatively pure sine-wave output, and a very stable frequency.

\subsection{Power Amplifier Design}

As reviewed in Chapter II, many GaN-based PAs have been reported.. In this thesis, a single frequency power amplifier operating at $\mathrm{X}$-band is designed and realized, using a GaN discrete bare die transistor from Cree. This PA will be used to conduct the complete analysis of the reconfigurable frequency oscillator using GaN HEMT technology at X-band.

\subsubsection{PA Design Procedure}

\section{a) Cree's CGHV1J025D GaN Descrete Transistor Die}

Cree's CGHV1J025D GaN discrete transistor die is chosen as the active device to design the PA as well as the fixed frequency and the tunable frequency 
oscillators. The manufacturer provides a nonlinear model for the device, so DC and small signal analysis can be easy done using one of commercially available electromagnetic simulation tool. Again for this design, ADS Agilent was used as EM and schematic simulator.

From the datasheet [139] provided by Cree, the total gate length of the CGHV1J025D is $5 \mathrm{~mm}$ and specified power density of the device is around $5 \mathrm{~W} / \mathrm{mm}$. Therefore, it can provide approximately $25 \mathrm{~W}$ output power with $10 \mathrm{~dB}$ power gain in 9-11 GHz frequency band. However, these values are measured using on-wafer measurements so they do not include any circuit loss. A photo of the transistor is shown in Figure IV.38.

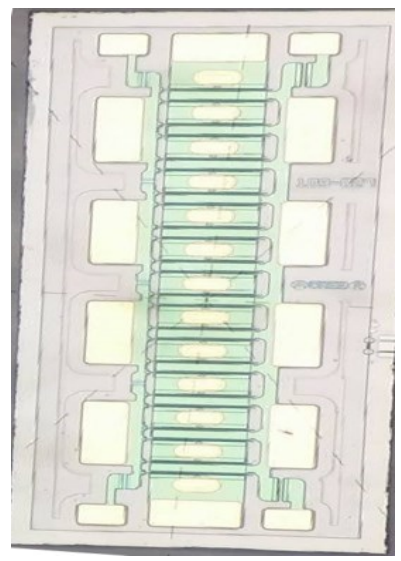

Figure IV.38: Photo of the CGHV1JO25D GaN Transistor Die

Choosing this device has been made on some purposes which are summarized as follow:

- The maturity of using this device in previous GaN PA works

- Its performances around X-band appears very interesting

- The cost is relatively moderate (\$75 US/Unit)

The GaN PA is implemented with matching circuits on a Neltec NX9300 substrate which its proprieties are: 
- Dielectric Constant of 3

- Thickness of $0.786 \mathrm{~mm}$

- Loss Tangent of 0.0006 to $0.0011 @ 10 \mathrm{GHz}$

- Thermal Conductivity of $0.381 \mathrm{Watts} / \mathrm{m} / \mathrm{K}$

By selecting a material with a $\mathcal{E r}$ of 3 allows to design a $50 \mathrm{Ohms}$ TL of width of $2 \mathrm{~mm}$ at $10 \mathrm{GHz}$ which falls in Cree's device's width, $1.9 \mathrm{~mm}$. Since wire-bond technique will be used between transistor die and matching network substrates to provide connection, a TL of width of $2 \mathrm{~mm}$ will be good to dispatch symmetrically the wires bond over the entire surface (see Figure IV.39).

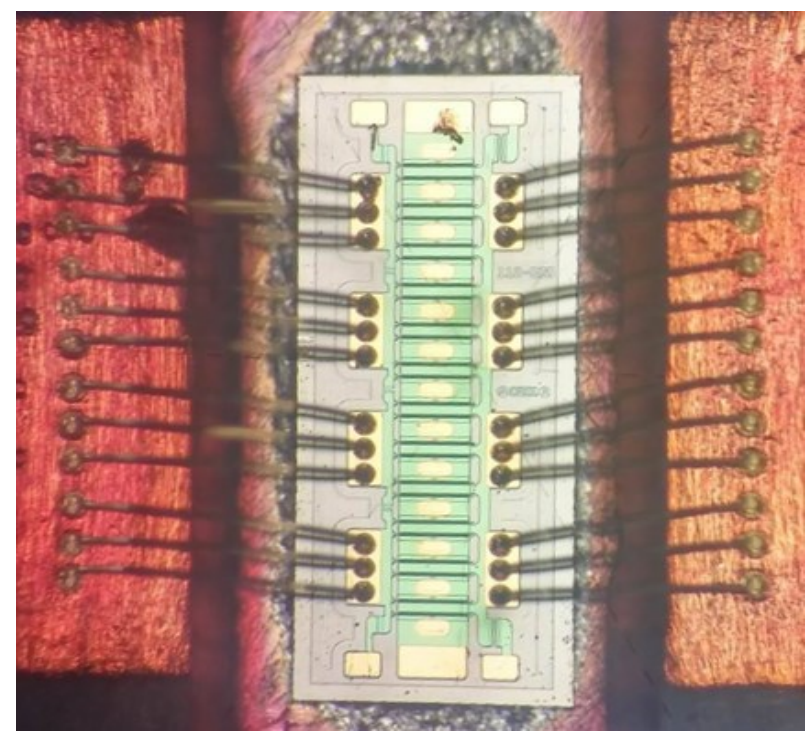

Figure IV.39: 3D Bond wire model.

\section{b) PA Operating class, DC and Stability Analysis}

Since a nonlinear model of the transistor is available, this simplifies the design procedures such as DC analysis, stability analysis and simulations. Based on the provided model, the PA is designed and biased in class $\mathrm{AB}$ for $\mathrm{Vds}$ of $40 \mathrm{~V}$ and Vgs of $-2.5 \mathrm{~V}$ corresponding to a drain quiescent current Idq of $240 \mathrm{~mA}$. As already said, the designed PA will be used in a feedback frequency reconfigurable oscillator. This topology of oscillator is mainly based on a PA that may be biased in class A, B, 
$\mathrm{C}$ or $\mathrm{AB}$ since a gain more than $1 \mathrm{~dB}$ is reached, this allowing to satisfy the first oscillation condition. For this $\mathrm{PA}$, the class $\mathrm{AB}$ operation was chosen.

Figure IV.40 shows the simulated I-V curve of the device. The FET transistor is biased at $\mathrm{Vgs}=-2.5 \mathrm{~V}$ for class $\mathrm{AB}$ operation with a bias current which is approximately $10.2 \%$ of maximum Ids. The high Vds allowed by Cree is about $40 \mathrm{~V}$ and the device's breakdown voltage is about $125 \mathrm{~V}$. As reported in datasheet [139], the active device is unconditionally stable above $12 \mathrm{GHz}$ and potentially unstable otherwise. Therefore, a series gate resistor of a small value around $0.175 \Omega$ is required by Cree to get device stable at $10 \mathrm{GHz}$. Since, S-parameter response of the device is function of the bias points, stability factors must be simulated at the operating bias conditions. This step in designing the PA is to determine with the small signal model whether the FET transistor is unconditionally stable or potentially unstable. This can be easily analyzed using the stability factor (K-factor) and stability measure delta $(\Delta)$. $\Delta>0$ and $K>1$ are necessary and sufficient conditions for stability. The equations for $\mathrm{K}$-factor and $\Delta$ are defined as follow:

Equation (23)

$$
K=\frac{1+|\Delta|^{2}-|S(11)|^{2}-|S(22)|^{2}}{2|S(12) S(21)|}
$$

Equation (24)

$$
\Delta=S(11) S(22)-S(12) S(21)
$$

However, there is an inverse proportionality between $\mathrm{k}$-factor and maximum available gain (MAG) so that $\mathrm{k}$-factor must be barely higher than 1 in the frequency band of interest but as large as possible out of band to have high gain and to prevent out of band oscillations. Stability analysis must be done from very low frequencies to very high frequencies to observe potentially unstable regions. The simulated K-factor and $\Delta$ results are shown in Figure IV.41. 
So a series resistor of $1.45 \Omega$ [139] was required for this design to get device stabilized. Therefore, this stability resistor is thought as a part of the input matching circuit.

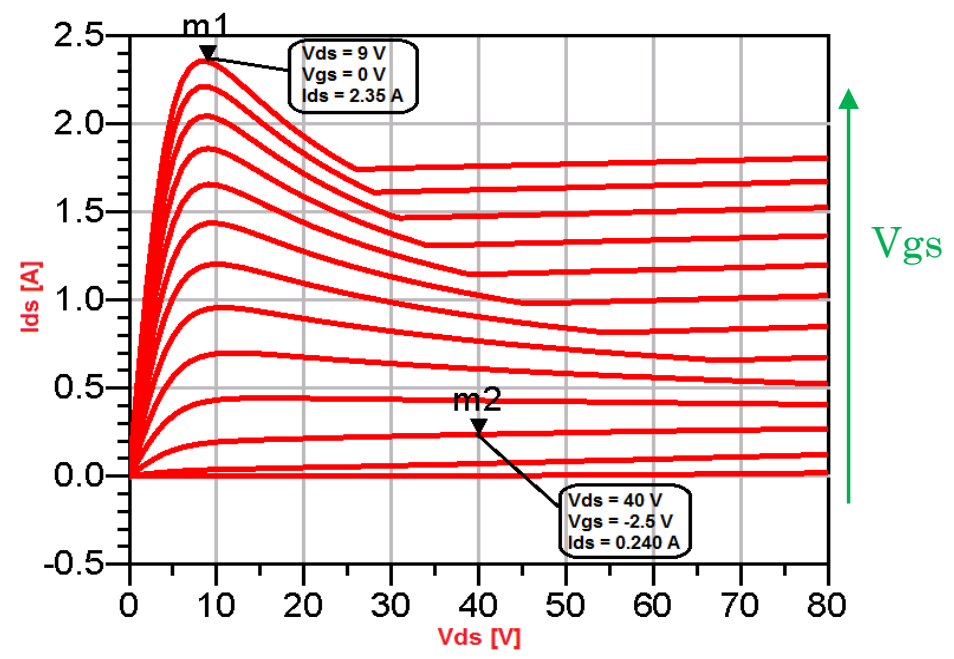

Figure IV.40: Simulated I-V Curves of the transistor

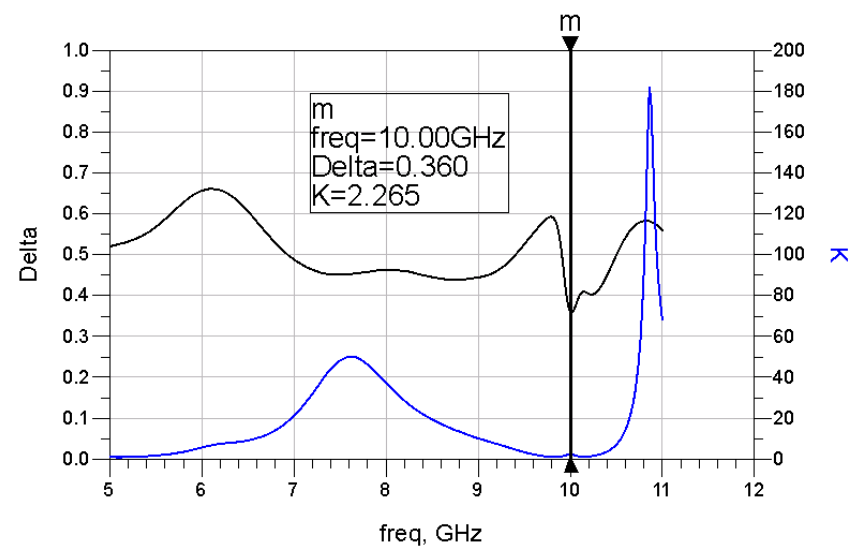

Figure IV.41: K-factor and Delta

\section{c) Bias Network}

The bias network is designed by using a quarter-wavelength ( $\mathrm{X} / 4)$ associated with a radial stub instead of a decoupling capacitor to avoid more wires bond. Radial stub should be properly designed at the operating frequency to provide a very low input impedance which is equivalent to $R F$ short circuit, and then this 
short circuit will be transformed to $R F$ open circuit by a properly designed $\lambda / 4$ transmission line. This means the DC voltage can go through the $\lambda / 4$ transmission line to bias the transistor, while $\mathrm{RF}$ signal at $10 \mathrm{GHz}$ see an open circuit due to the $\lambda / 4$ transmission line thus allowing decoupling the circuit from the DC supply (Figure $I V$.43). In this PA design, two bias networks can be cascaded in series to achieve more RF to DC decoupling (see Figure IV.43).

Some decoupling capacitors have to be placed to isolate both bias DC at drain and gate (Vds and Vgs). For this, GX03 ultra broadband decoupling capacitors from the market were chosen. This capacitor has good performances in term of insertion loss and return loss over a broad band range from $16 \mathrm{KHz}$ to $40 \mathrm{GHz}$. At 10 $\mathrm{GHz}$ this capacitor has an insertion loss of $-0.1 \mathrm{~dB}$ and a return loss value better than $-25 \mathrm{~dB}$.

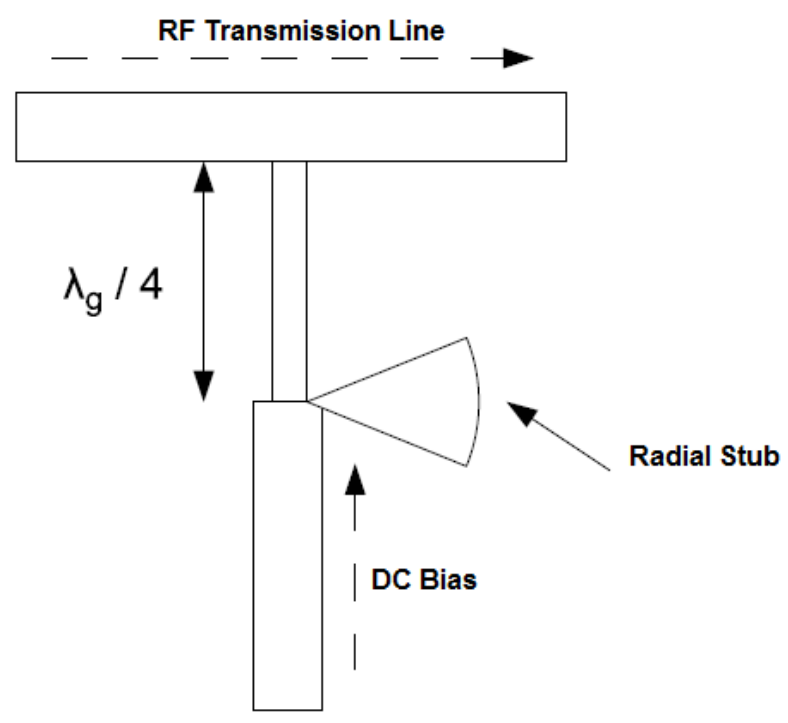

Figure IV.42: A typical distributed bias network with radial stub

\section{d) Matching Network}

As given in datasheet and verified with simulations, optimum load and source impedances of the Cree's active device at $10 \mathrm{GHz}$ for $\mathrm{Vds}$ of $40 \mathrm{~V}$ and Idq of $240 \mathrm{~mA}$ are, respectively, 0.866-j0.461 $\Omega$ and 3.08-j8.705 $\Omega$. In addition, the matching 
networks were designed by adding parallel open stubs at the input and output of PA. These open stubs transform both optimum load and source impedances to provide maximum gain matching. Table IV.3 summarizes the dimensions of the used stubs. The fabricated PA prototype is shown in Figure IV.43. GaN power device are soldered on heat-sink plate with high thermal conductivities. It allows to transfer the heat generated by PA and provides an infrastructure to mount the PCB and the RF connectors. Copper was used for the reason that it has a higher thermal conductivity then other ordinary material such as aluminum, however copper makes the prototype more heavier. Also the transistor was mounted directly to the $\mathrm{Cu}$ plate using an epoxy glue.

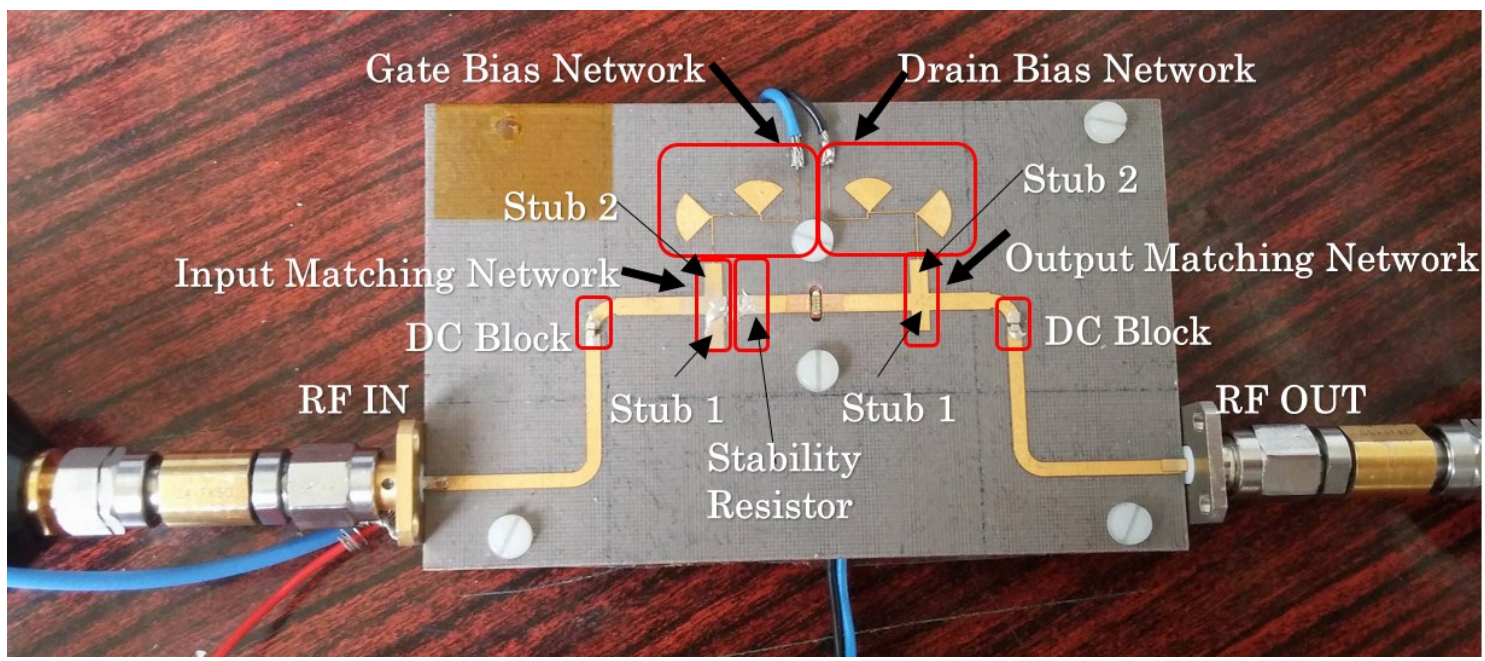

Figure IV.43: Fabricated PA under Test

Table IV.3: Dimensions of Stubs

\begin{tabular}{|c|c|c|c|}
\hline & & Input Matching Network & Output Matching Network \\
\hline \multirow[b]{2}{*}{ Stub 1} & Length & $3.8 \mathrm{~mm}$ & $2.24 \mathrm{~mm}$ \\
\hline & Width & $2 \mathrm{~mm}$ & $2 \mathrm{~mm}$ \\
\hline \multirow[b]{2}{*}{ Stub 2} & Length & $3.66 \mathrm{~mm}$ & $3.75 \mathrm{~mm}$ \\
\hline & Width & $2 \mathrm{~mm}$ & $2 \mathrm{~mm}$ \\
\hline
\end{tabular}

\subsubsection{PA Simulated Results}

Since this PA design will used as a feed-back oscillator, large signal simulation will not be conducted. So Small signal simulation results of the PA are 
given in Figure IV.44. It is showing that gain of the amplifier is around $6 \mathrm{~dB}$ which satisfy the first oscillation condition (> $1 \mathrm{~dB}$ ) at $10.1 \mathrm{GHz}$. Moreover, input and output return loss value of the PA is better than $-12 \mathrm{~dB}$ this clearly shows that a good matching is achieved. In addition, $\mathrm{S} 12$ is lower than $-12 \mathrm{~dB}$.

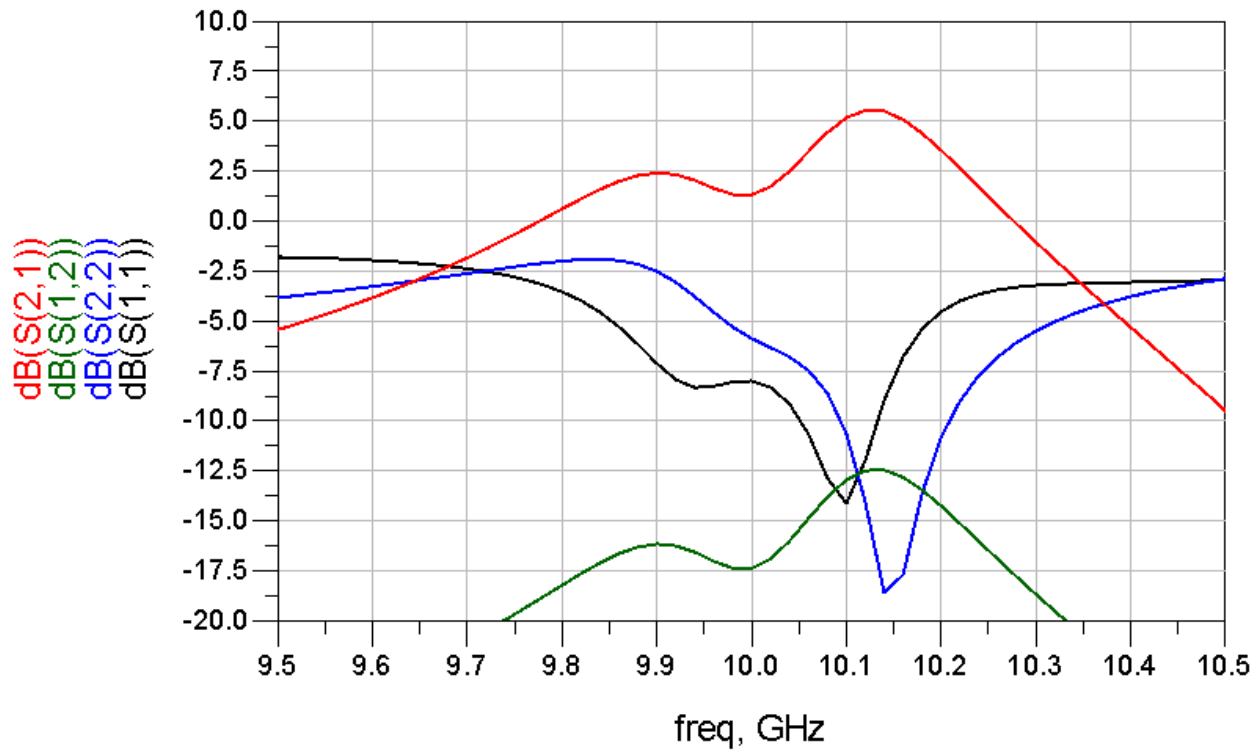

Figure IV.44: Simulated Small Signal S-Parameters

\subsubsection{PA Measured Results}

As a recall during the measurements, the $\mathrm{PA}$ is biased in Class $\mathrm{AB}$ mode whose quiescent drain current about $240 \mathrm{~mA}$ should be achieved for Vgs of $-2.5 \mathrm{~V}$ and Vds of 40 V.

Figure IV.45 represents the small signal measurement results of the fabricated PA. It is observed that the PA does not have any gain, a value of $-5.3 \mathrm{~dB}$ was observed for $\mathrm{S} 21$ at $9.6 \mathrm{GHz}$, even good match is achieved at the input ( $\mathrm{S} 11=-16.4 \mathrm{~dB})$ as well as the output ( $\mathrm{S} 22=-11.6 \mathrm{~dB}$ ) as can be seen. It seems that this PA does not works as expected. As shown in the picture illustrated in Figure IV.46, the desired quiescent drain current of $260 \mathrm{~mA}$ is achieved but at a lower Vds of $18 \mathrm{~V}$.

During the measurement, Vgs was fixed at $-2.5 \mathrm{~V}$ and then we start increasing slightly the Vds to get the wanted Ids current. Once the Vds gets higher than $19 \mathrm{~V}$, it was 
observed that the Vgs starts getting changed alone randomly. The Vds starts to control the Vgs value and we lost completely the control over the Vgs. We could not very understand this weird behaviour. It is known that when there is a drop value in gain this means that the PA is not stable. Maybe a little bit more complicated stability circuits than a simple series resistor recommended by CREE is required to make sure that the device is stable.

The transistor model of the CGHV1J025D provided by CREE Inc. only has one input port and one output port, despite the fact that the transistor itself is constituted from twelve cascaded single transistors, so CGHV1J025D has twelve input ports and output ports. Unfortunately a distributed transistor model that can allow to check if all the single devices work well was not available, also it was impossible to check if the mounded device was damaged or not. Due to our limited funds, we could not offer another fabrication prototype. In addition, to redo another fabrication will be considered as very time consuming.

Since this PA is planned to be used as a feed-back power oscillator, it will be very difficult to achieve our objective and make the oscillator works well. Nevertheless, the study of the two oscillators has been done and presented in the following section.

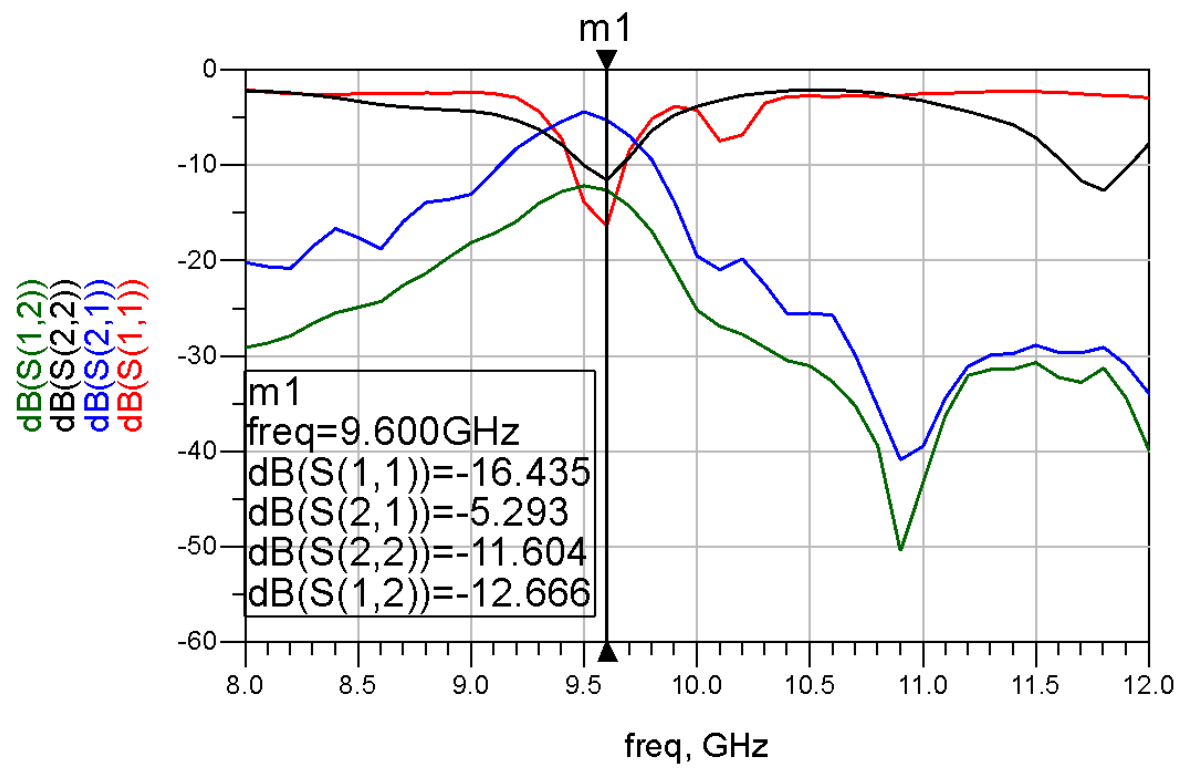

Figure IV.45: Measured Small Signal S-Parameters 

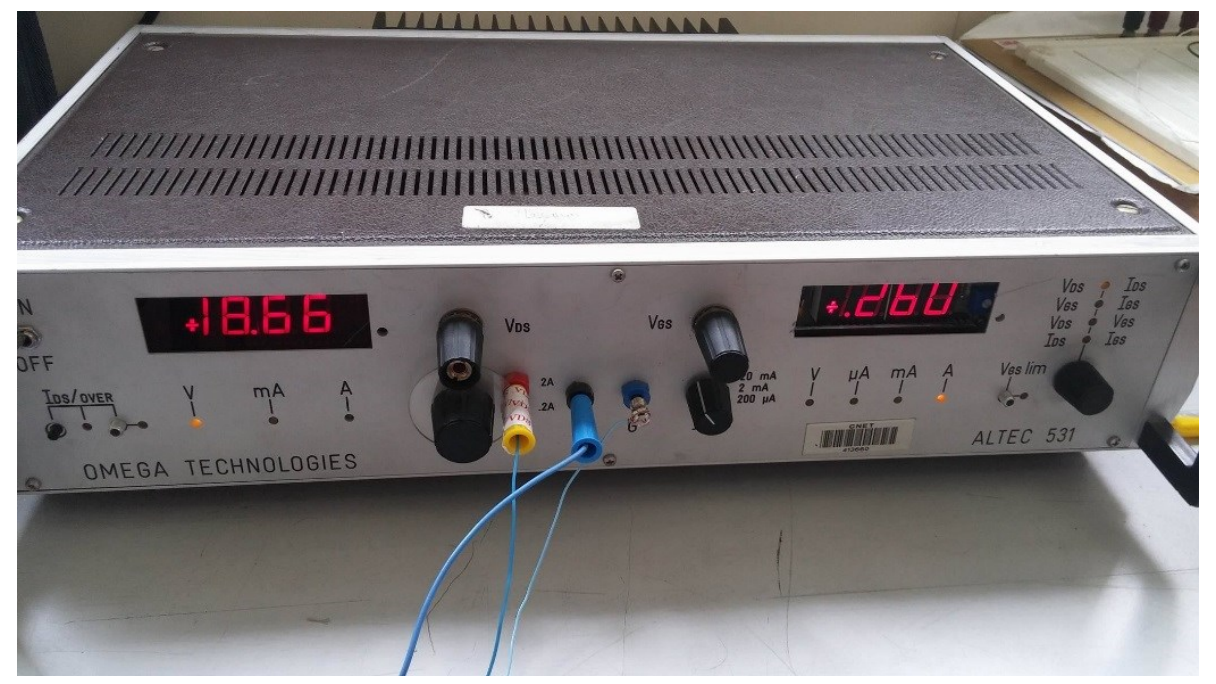

Figure IV.46: Ids of $260 \mathrm{~mA}$ at $\mathrm{Vds}$ of $18.6 \mathrm{~V}$ and $\mathrm{Vgs}$ of $-2.5 \mathrm{~V}$

\section{Oscillator Design}

\subsection{Start-up Oscillation Condition}

Under proper bias conditions, a feedback oscillator start to oscillate from the RF noise level without a real input RF signal. This noise presents at the input of the oscillator or generated by the active and passive devices in the oscillator, is responsible for starting-up the oscillation regime. As the power supply is turned on, noises will be shown up and then oscillation will grow in amplitude. Although, this noise is characterized by a broadband spectrum but only noise at the oscillation frequency will be amplified. The gain of the amplifier and feedback loop must be equal or greater than one at the desired oscillation frequency, while the phase shift through the loop must be zero or some integer multiple of $2 \pi$. These are known as Barkhausen Criterion [140] of oscillations and are required to get the oscillating regime process ongoing.

Usually the designed loop gain magnitude is slightly larger than unity at the desired frequency of oscillation because if exact unity is designed, a slight 
reduction in gain would result in oscillations that decays to zero. So the oscillation conditions in open loop can be summarized as:

$$
\begin{aligned}
& S_{21} \gg 1 \\
& S_{11}<<1 \\
& \left.\operatorname{Arg}\left(S_{21}\right)=0 \_ \text {or_[n. } 2 \pi\right]
\end{aligned}
$$

When designing this kind of oscillator topology, it is preferred to proceed firstly by get the matching done $\left(S_{11}<<1\right)$, then secondly the gain and the phase can be optimized to satisfy the oscillation conditions.

To ensure that the design meets the necessary conditions for oscillation, a small signal gain analysis has to be done before connecting the feedback network to the amplifier's input. This analysis is mostly called open-loop analysis where gain and phase have to satisfy Barkhausen Criterion of oscillations. In addition, when dealing with oscillator designs, closed-loop analysis is also required to explore the oscillator's performances such as frequency and power of oscillation as well as phase noise. This analysis is conducted once the feedback loop is connected to the amplifier's input terminal. Oscillator's performances at both open-loop and closedloop are discussed here for FFO as well as for TFO.

The goals of the two discussed oscillators designs is firstly to fulfill the indepth progress of GaN oscillator design, and to achieve an high oscillator output power at $10 \mathrm{GHz}$ with low phase noise. Also a frequency reconfigurability over X-band is another goal of this conducted study. The configuration of both the FFO and TFO designs is shown, respectively, in Figure IV.47 and Figure IV.54. The circuits was simulated using ADS. Small signal and harmonic simulations were performed. 


\subsection{Phase Noise in Oscillator}

Phase noise is one of the most important figures of merit of a signal generating device and it is often the limiting performance metric. The basic concept of phase noise involves frequency stability, which is defined as the degree to which an oscillating source produces the same frequency throughout a specific period of time. Phase noise is generally specified in $\mathrm{dBc} / \mathrm{Hz}$ at a given offset frequency for a particular carrier. Therefore, phase noise can be found by measuring the ratio of the power spectral density $(1-\mathrm{Hz}$ bandwidth) at a given offset frequency to the total power at the carrier frequency. In the literature, it is common practice to refer to the values of phase noise as "close-in" for the frequency offset range of $1 \mathrm{~Hz}$ to $100 \mathrm{~Hz}$. That is, "close in phase noise" refers to the phase noise close to the carrier, less than $1 \mathrm{kHz}$ away. Similarly, "far out" phase noise commonly refers to values $1 \mathrm{MHz}$ or more from the carrier. That is, "far out phase noise" refers to the phase noise far from the carrier, more than $100 \mathrm{kHz}$ away. The mid-range region, $1 \mathrm{kHz}$ to $100 \mathrm{kHz}$ offset, especially for signal generator phase noise, is sometimes referred to as the pedestal region.

It is well known that depending on the application, the offset frequency is selected. Here in this designed oscillator, phase noise is simulated at three offset frequencies, such as $10 \mathrm{KHz}, 100 \mathrm{KHz}$ and $1 \mathrm{MHz}$. As mentioned, the idea behind the design of this oscillation is to verity the performance of using the developed GaN varactors, so these offset frequencies were selected only because most of the reported GaN oscillator designs found in the literature also use these three selected offset frequencies, and we assumed that for a good comparison, the noise phase should be simulated also at the same offset frequencies. 


\subsection{Fixed Frequency Oscillator (FFO)}

\subsubsection{Simulation Results}

Figure IV.47 illustrates the final realized FFO prototype. As can be seen in Figure IV.48, the open-loop analysis has been done and simulated results are displayed. An open-loop gain around $6 \mathrm{~dB}$ with a good matching at the input is achieved at $10.12 \mathrm{GHz}$. While the signal phase is around $0^{\circ}$ at this frequency. These values satisfy the required oscillation conditions.

Once the open-loop simulations are done, the feedback network has been connected to the input and a $50 \Omega$ port was used as oscillator's output to conduct the second analysis, closed-loop simulation. Figure IV.49 shows the simulated spectrum for the FFO providing $34.7 \mathrm{dBm}$ output power at $10.01 \mathrm{GHz}$ oscillation frequency. The circuit is biased at Vds of $40 \mathrm{~V}$, Ids of $240 \mathrm{~mA}$, and Vgs of $-2.5 \mathrm{~V}$. The power levels of the second and third harmonics appear in same Figure IV.49. The second harmonic was typically better than $40 \mathrm{dBc}$ while the third one was so small as $50 \mathrm{dBc}$. In Figure IV.50 and Figure IV.51 a pure sinusoid was generated at the output of the FFO. An output voltage of $17.3 \mathrm{~V}$ was achieved.

Figure IV.52 shows the results of phase noise at $10 \mathrm{KHz}, 100 \mathrm{KHz}$ and 1 $\mathrm{MHz}$ offset where values of $-103 \mathrm{dBc} / \mathrm{Hz},-110 \mathrm{dBc} / \mathrm{Hz}$, and $-143 \mathrm{dBc} / \mathrm{Hz}$ were simulated, respectively. 


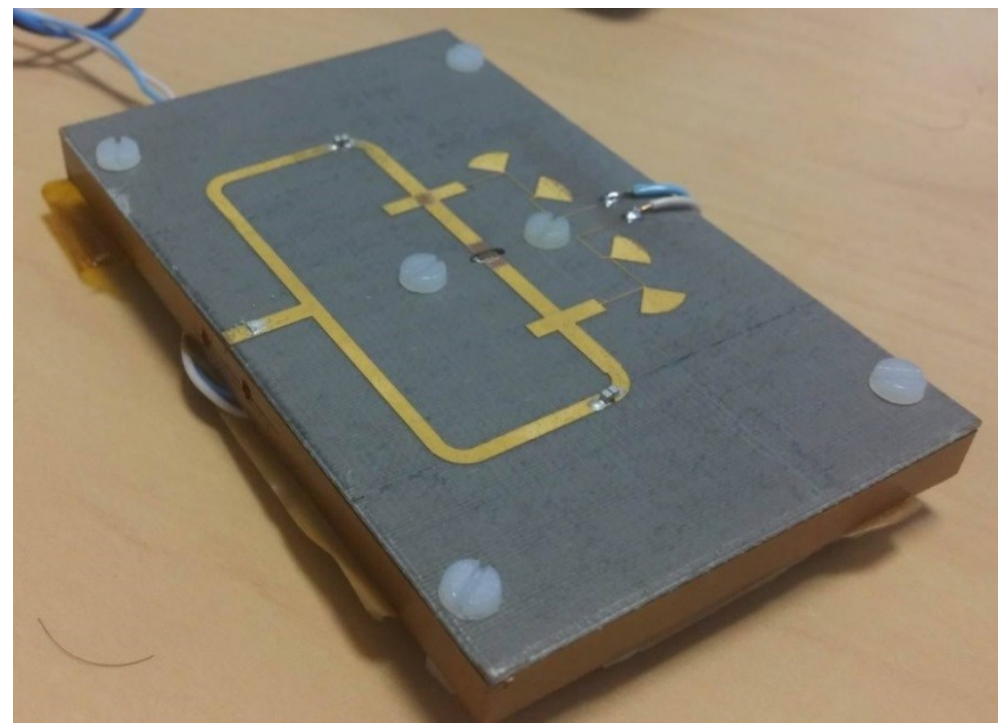

Figure IV.47: Fixed Frequency Oscillator Fabricated Prototype

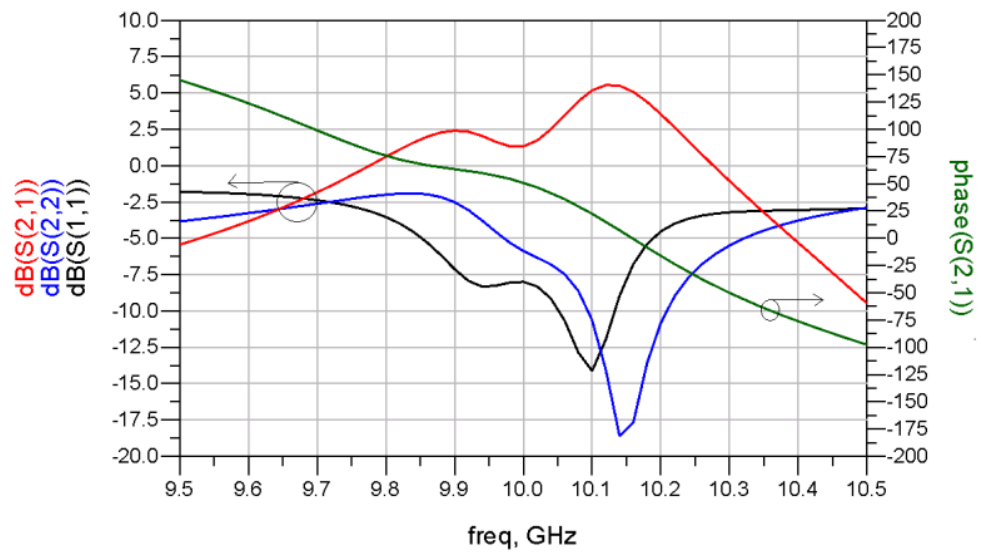

Figure IV.48: Small Signal Simulation Results (Open-Loop)

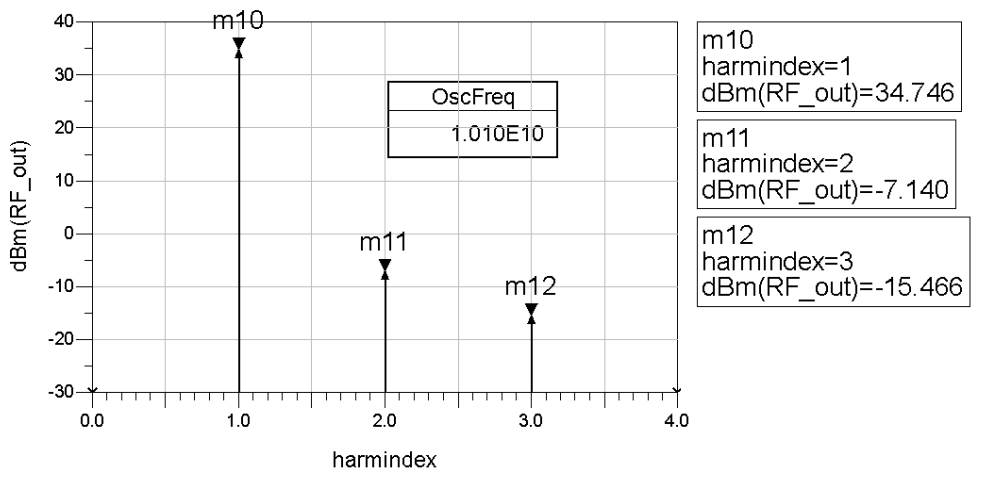

Figure IV.49: Harmonics Power Simulation of the FFO 


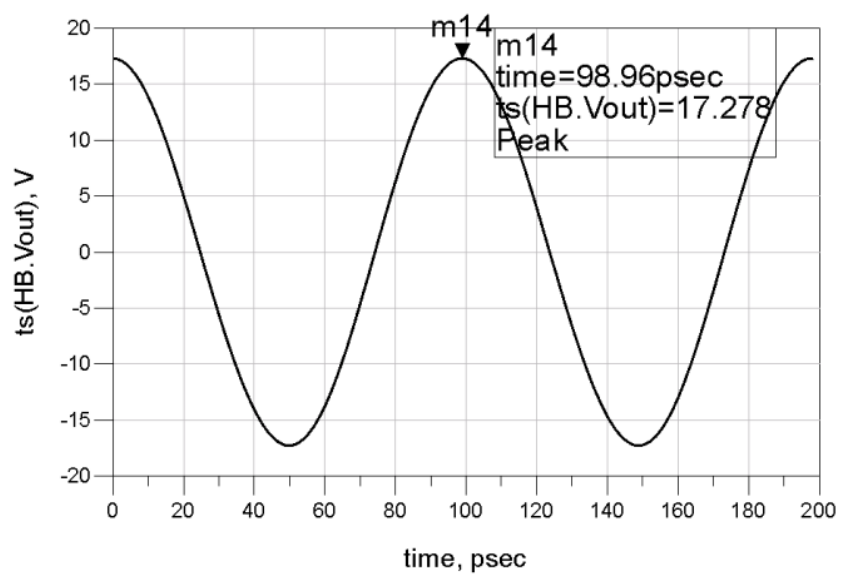

Figure IV.50: Pure Voltage Sinusoid at the Output of the FFO

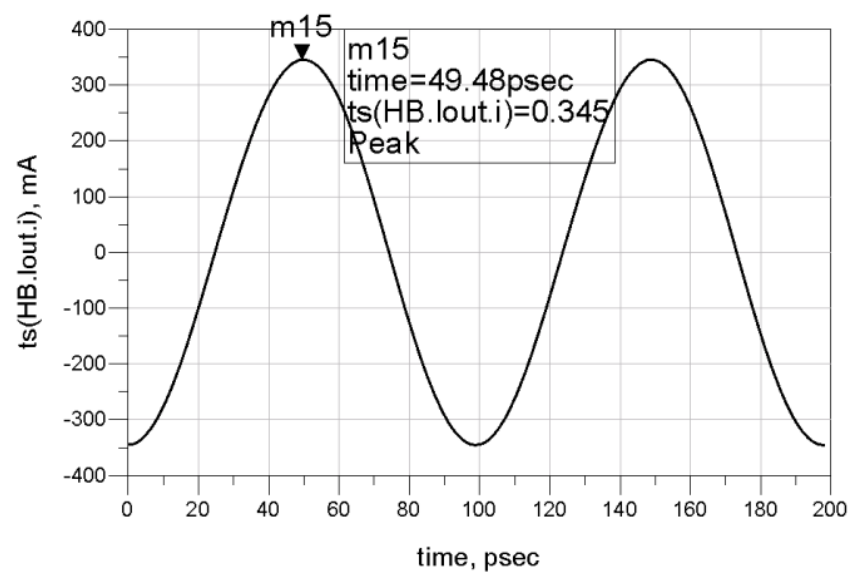

Figure IV.51: Pure Current Sinusoid at the Output of the FFO

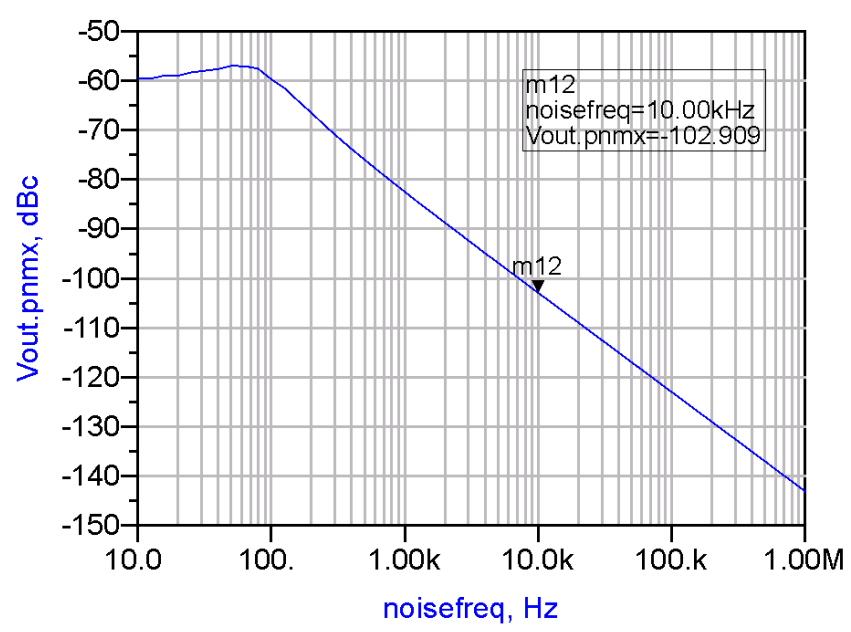

Figure IV.52: Simulated Phase Noise of the FFO 


\subsubsection{Measurements Results}

First of all, to prevent damage on laboratory equipment a $20 \mathrm{~dB}$ attenuator was added at the input of the spectrum analyzer. With Matlab, this attenuated power was recovered and real measured oscillation power is depicted in Figure IV.53.

As can be seen there are a lot of oscillation frequencies below $10 \mathrm{GHz}$, this means that the feedback network do not filter these frequencies. As already reported, the PA that is used here for this oscillator did not work as intended from simulation, so it is obvious that the FFO does also not work as expected. At the operating frequency of $10 \mathrm{GHz}$, it is observed that a peak of a power of $-10 \mathrm{dBm}$ is measured while the highest power peak of $26 \mathrm{dBm}$ was observed at very low frequency around $500 \mathrm{MHz}$ as reported in Figure IV.49. These results are recorded at bias points of Vgs of $18 \mathrm{~V}$ and $\mathrm{Vgs}$ of $-2.5 \mathrm{~V}$ where an Ids current of $260 \mathrm{~mA}$ was achieved. The same problems observed for the PA with the biasing are also observed during the FFO measurements. It was noted that the Vds starts controlling the Vgs.

Due to the fact that the design did not work as intended from simulations, measurements of the power levels of the second and third harmonics as well as of the phase noise are not reported.

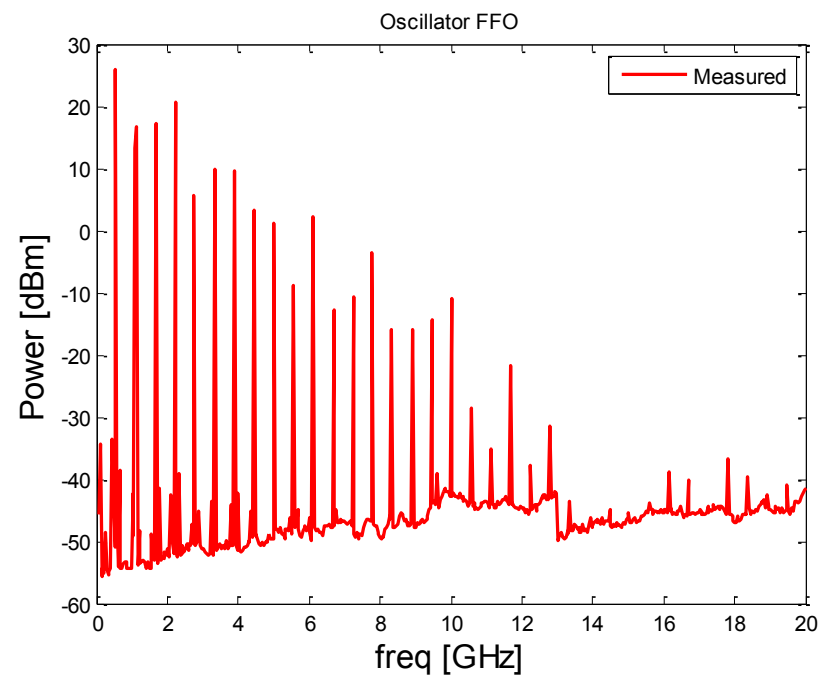

Figure IV.53: Harmonics Power Measurement of the FFO 


\subsection{Tunable Frequency Oscillator (TFO)}

\subsubsection{Simulation Results}

In order to tune the oscillation frequency, the feedback loop of the previous FFO design was symmetrically loaded by two GaN varacator diodes to design a frequency reconfigurability GaN oscillator. A small signal model of these varactors has been developed and presented in Chapter III. Varactor diodes are commonly used in $\mathrm{RF} /$ microwave oscillators, enabling the tuning of the oscillation frequency which is controlled by the reverse applied voltage. The tuning voltage across the varactor is swept from $-5 \mathrm{~V}$ to $-3.7 \mathrm{~V}$. In Figure IV.54 the fabricated TFO is shown.

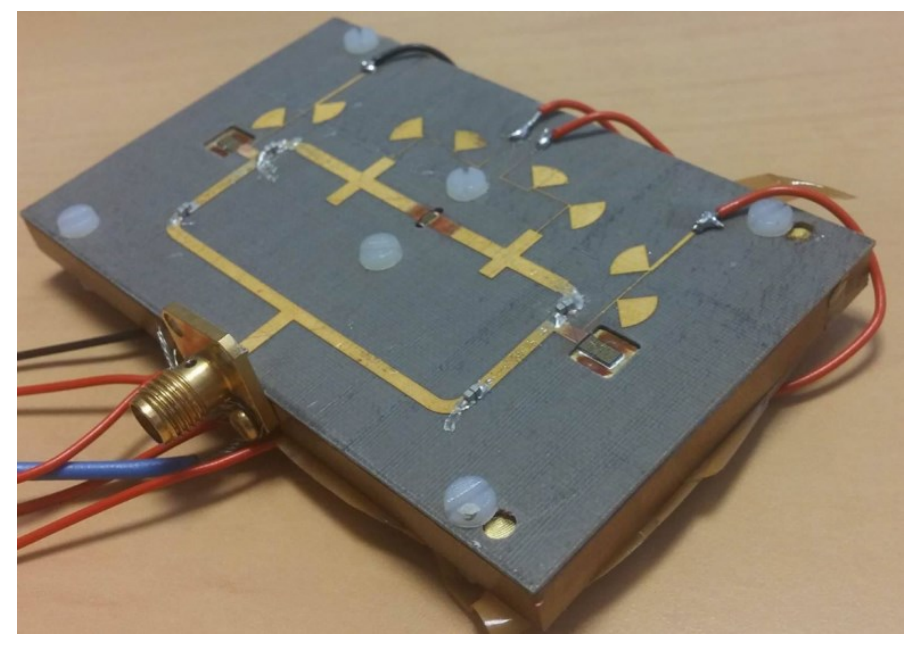

Figure IV.54: Tunable Frequency Oscillator fabricated Prototype

Figure IV.55 presents the oscillation frequency versus varactor bias voltage $V_{G}$ of the TFO. In terms of variation, it is seen that the frequency oscillation versus bias voltage curve exhibits an inverse behavior of $\mathrm{C}_{\mathrm{EQ}}$ versus $\mathrm{V}_{\mathrm{G}}$ presented in chapter III. A minimum oscillation frequency of $7.8 \mathrm{GHz}$ was achieved when $\mathrm{C}_{\mathrm{EQ}}$ is at its maximum over range (1) for $\mathrm{V}_{\mathrm{G}}$ above $-3.5 \mathrm{~V}$, and a maximum oscillation frequency of $8.6 \mathrm{GHz}$ was simulated when $\mathrm{C}_{\mathrm{EQ}}$ is at its minimum over range (3) for $\mathrm{V}_{\mathrm{G}}<-4.5 \mathrm{~V}$. While, in range (2) from $-5 \mathrm{~V}$ to $-3.7 \mathrm{~V}$, the oscillation frequency values change significantly and a tuning bandwidth of $0.8 \mathrm{GHz}$ is obtained, corresponding 
to a tuning range about $10 \%$. This rapid change corresponds to the variation of $\mathrm{C}_{\mathrm{EQ}}$ over range (2).

In Figure IV.56 the output power versus bias voltage $\mathrm{V}_{\mathrm{G}}$ of the TFO is illustrated. It can be seen that the power is about $20.5 \mathrm{dBm}$ and almost stable over all bias levels with a slight variation over range (2) where $\mathrm{V}_{\mathrm{G}}$ is between $-5 \mathrm{~V}$ and $3.7 \mathrm{~V}$. This drop in power variation can be due to the variation in equivalents series resistor of varactor as presented in previous Chapter III. Compared to the output power of the FFT which is about $34.7 \mathrm{dBm}$, the output power of TFO is decreased to 20.5. This can be due the RF losses of varactors used for tuning purpose.

Simulated phase noise versus bias voltage across varactors at three offset frequencies for the TFO is shown in Figure IV.57. For all bias points, phase noise values of $-92 \mathrm{dBc} / \mathrm{Hz},-112 \mathrm{dBc} / \mathrm{Hz}$ and $-132 \mathrm{dBc} / \mathrm{Hz}$ are met at $10 \mathrm{KHz}, 100 \mathrm{KHz}$ and $1 \mathrm{MHz}$, respectively. Also, due to the variation of $\mathrm{R}_{\mathrm{EQ}}$, a slight variation of phase noise is noticed from $-5 \mathrm{~V}$ to $-3.7 \mathrm{~V}$.

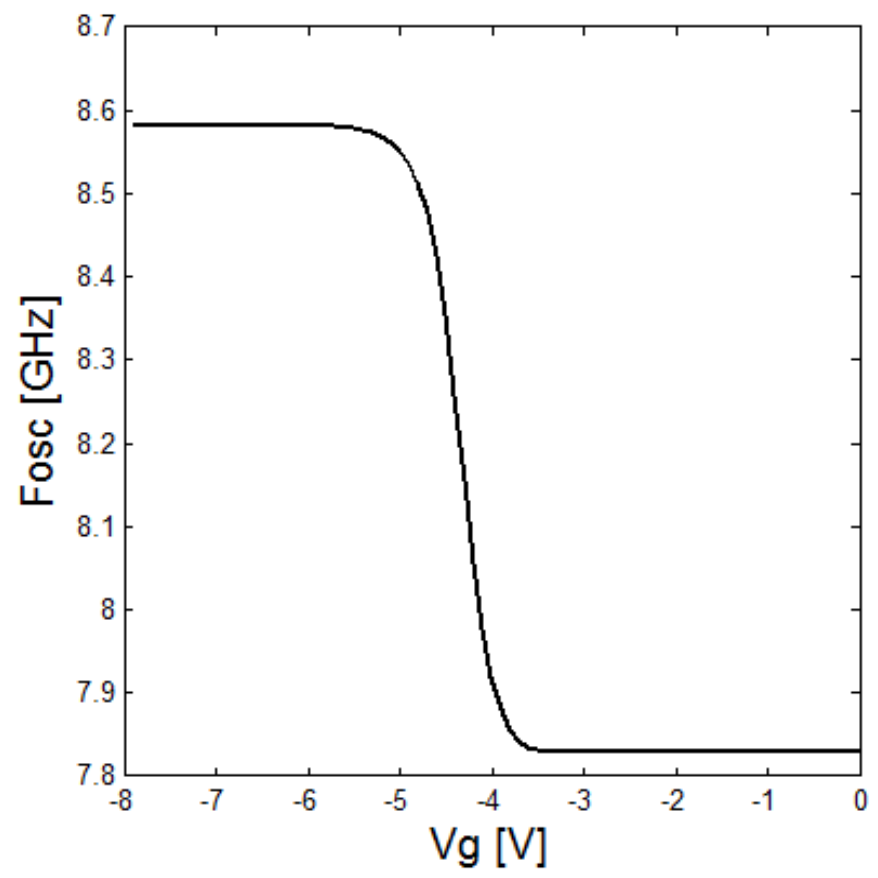

Figure IV.55: Oscillation frequency versus gate voltage 


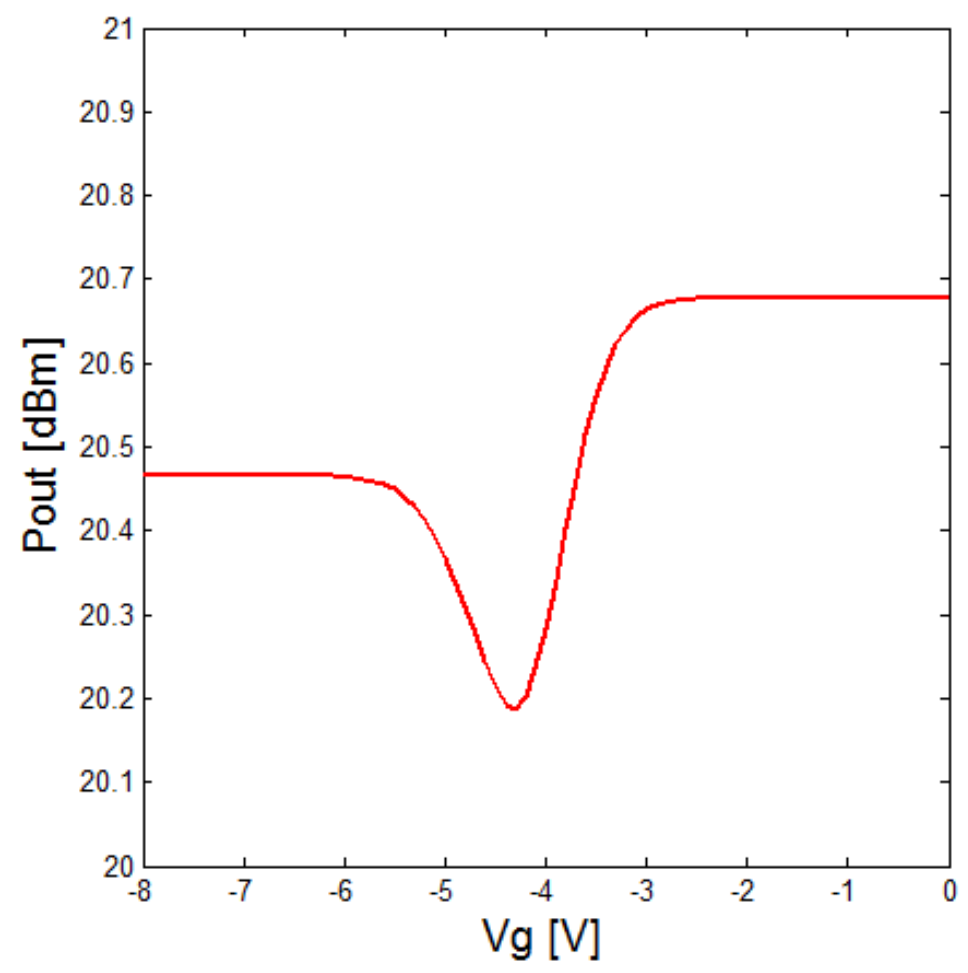

Figure IV.56: Output power versus gate voltage at fundamental frequency

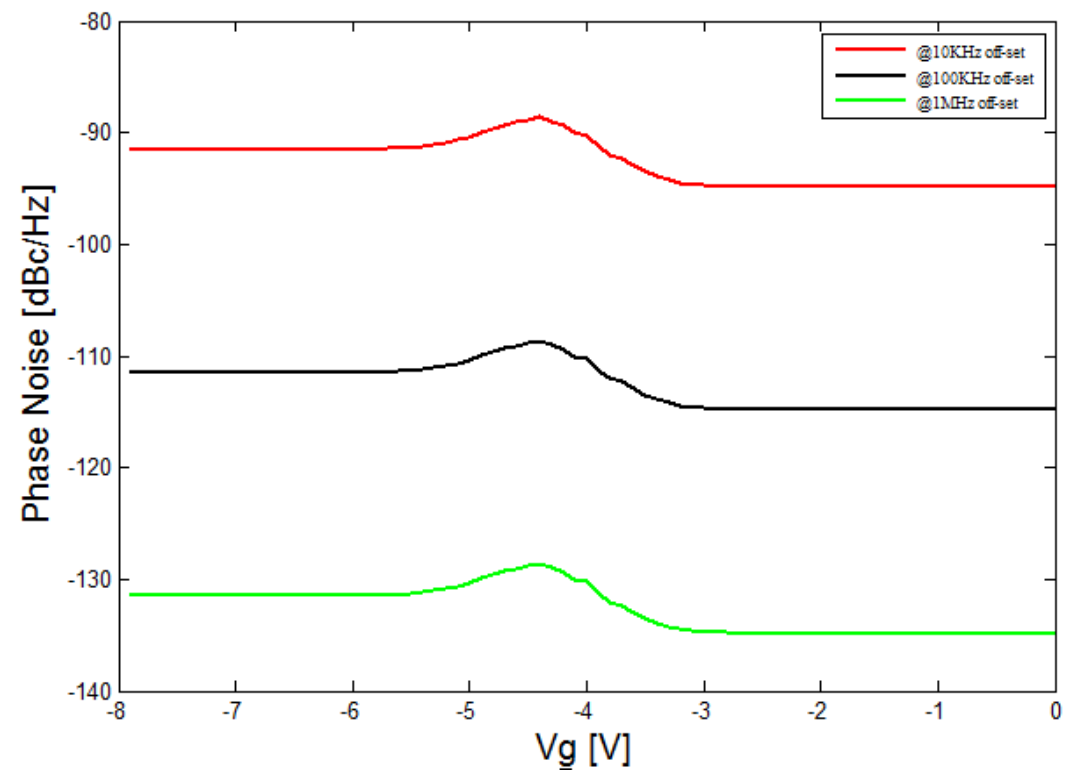

Figure IV.57: Phase Noise versus gate voltage at $10 \mathrm{KHz}, 100 \mathrm{KHz}$, and $1 \mathrm{MHz}$ 


\subsubsection{Measurement Results}

For the measurements part, the same remarks as for the PA and the FFO have been observed for the TFO design.

In this section, two GaN oscillator designs based on feedback topology have been presented for high RF power source. First, the power amplifier and optimized feedback loop were designed and fabricated using a Cree's discreet active FET device and NELTIC9300 substrate for the PCB design. The amplifiers has an open-loop gain of $6 \mathrm{~dB}$, good return loss at input and output better than $-12.5 \mathrm{~dB}$, and phase of $0^{\circ}$ at $10.12 \mathrm{GHz}$.

Secondly, the feedback loop was closed to design the first oscillator (FFO). Phase noise about - $110 \mathrm{dBc} / \mathrm{Hz} @ 100 \mathrm{kHz}$ was simulated at the bias Vds of $40 \mathrm{~V}$ and Ids of $240 \mathrm{~mA}$ while the output power obtained for the FFO is $34.7 \mathrm{dBm}$ at center frequency of $10.12 \mathrm{GHz}$. The second and third harmonics were very low and typically better than $40 \mathrm{dBc}$. These simulations results are favorably compared to the best reported performances of high power GaN oscillator [129]-[134]. In [129] Using a GaN HEMTs with a gate length of $0.15 \mu \mathrm{m}$ process, an output power of $35 \mathrm{dBm}$ between 8.5 and $9.5 \mathrm{GHz}$ was achieved at $V d s=30 \mathrm{~V}$ while the phase noise was estimated to be $-87 \mathrm{dBc} / \mathrm{Hz}$ at $100 \mathrm{kHz}$ offset.

Finally, a tunable frequency GaN oscillator (TFO) is designed by loaded symmetrically the feedback loop. For tuning purpose, GaN varactor were used. The oscillation frequency values change significantly and a tuning bandwidth of $0.8 \mathrm{GHz}$, corresponding to a tuning range about $10 \%$ was achieved. The output power is about $20.5 \mathrm{dBm}$ and almost stable over all bias levels with a slight variation over range (2) while a good phase noise value of $-112 \mathrm{dBc} / \mathrm{Hz}$ is met at $100 \mathrm{KHz}$. However, the output power was not impressive compared to FFO design, being only $20.4 \mathrm{dBm}$ and $20.9 \mathrm{dBm}$ at best. This drop of power can be explained as there is a RF power consumption of varactors due to the presented high equivalent series resistor. 
These two reported oscillators prove clearly that GaN HEMT technology, with its high breakdown voltage, is a good promising choice for the high RF power sources. Unfortunately the measurements did not work as expected from simulation, this may be due to a difficulty to bias the active device and/or do not have the same ground at drain and gate.

\section{Frequency Reconfigurable Active Antennas}

\section{Review}

Active integrated antennas (AIAs) researches have shown unprecedented development along with growing interest for wireless systems applications [141]. The designs of AIAs are towards the designs that are more and more small, have the capability of operating at multi-frequencies [142] and of handling significant high power levels, which has been the greatest challenge. There are a plenty of techniques [141] in which antennas can be frequency tuned such as using switches, varying substrate properties [143] and using PIN diodes. Using varactors diodes [144] integrated with printed antennas can be another way to tune the antenna operating frequency. This technique based on varactors could be found in various types of reconfigurable active integrated antennas systems. However, active device such as varactor diodes presents some non-linear behaviors with high power RF signals that influence the performances of antenna.

As a result of advanced GaN based HEMT semiconductor devices such as good linearity, the designs of AIAs with high power handling capability and high frequency operation are now achievable. GaN-based HEMT semiconductor devices are no longer limited to radio frequency integrated circuit (RFIC) [145], having now been more attractive to AIAs systems [146].

Furthermore, reconfigurable AIAs, with high power/frequency, and excellent efficiency, are in great demand. In this chapter, firstly, a novel frequency 
tunable annular slot antenna using GaN HEMT varactor is presented for the first time. However, using GaN varactor requires a high voltage DC biasing. In addition, GaN varactor presents a rather high insertion loss caused by its series equivalent resistance resulting on reducing the radiation efficiency of antenna. A second frequency tunable aperture coupled slot antenna is then investigated to improve the gain.

Thirdly, a radiation pattern reconfigurable antenna is studied using different oriented printed antenna arrays and integrated with GaN HEMT switches to choose the beam direction of the complete antenna system.

\section{Reconfigurable Annular Slot Antenna using GaN Varactor}

\subsection{Antenna Design}

The used planar antenna is a simple element annular slot coplanar antenna. The structure of the realized antenna is illustrated in Figure IV.58. This antenna was already presented in [147]. The whole size of antenna is about $50 \times 50 \times 0.7$ $\mathrm{mm}^{3}$ and is printed onto glass substrate with $\mathrm{Er}$ of 5.7 and $\tan \delta$ of 0.006 at $2 \mathrm{GHz}$. CST Studio simulator was used as an EM simulator to design and simulate the antenna. First, the antenna itself was simulated and results are presented and discussed. Then the developed GaN varactor model was added to load the antenna. This allows to electronically adjust the antenna frequency by varying the varactor bias voltage applied at the gate. As will be shown, measurement results of the frequency tuning range showed good agreement with the simulations.

The frequency tunability of this antenna has been investigated firstly by loading a GaAs varactor diode bought from the market [147], after that the developed GaN varactor diode (W200L0.5) using GaN500v2 process is used instead. Since, the used GaN varactor provides various capacitive load to the antenna, the resonant 
frequency of the antenna can be tuned electrically by implementing the GaN varactor between radiation element and ground as it is shown in Figure IV.58. The varactor junction capacitance varies as a function of bias voltage as discussed in chapter III. These different capacitive loadings correspond to various electrical lengths of the antenna, and thus different resonant frequencies.

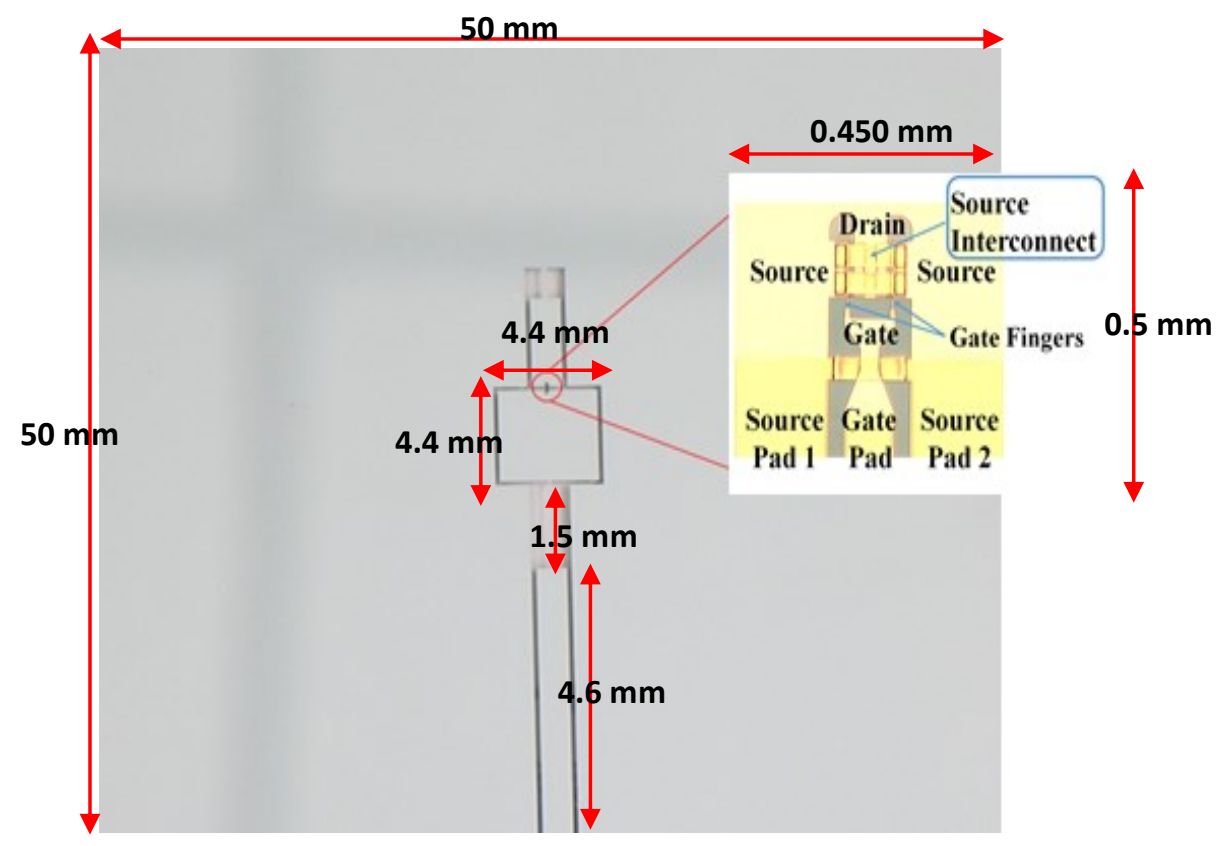

Figure IV.58: Printed antenna prototype with GaN varactor load.

\subsection{Simulated Return Loss and Radiation Pattern}

The antenna itself without the diode was firstly simulated and good matching was obtained at resonant frequency around $11 \mathrm{GHz}$ as shown in Figure IV.59. At this frequency, the simulated gain was about $4.6 \mathrm{~dB}$ as displayed in Figure IV.60. The radiation pattern of the antenna is simulated in the E-plane and H-plane for co-polarization (copol) at $11 \mathrm{GHz}$ and results are plotted in Figure IV.61. The E-plane and H-plane are normalized to the maximum gain of the antenna. Because the antenna structure is a coplanar simple element, it radiates in two opposite directions. In E-plane, the maximum beam direction occurs symmetrically around $30^{\circ}$ and $-30^{\circ}$ where a max gain was observed, this can be due to the diffraction 
of the limited size of the ground conductor and also some perturbation caused by the SMA connector used for the antenna excitation. The presented results of the antenna will be later experimentally verified and rediscussed.

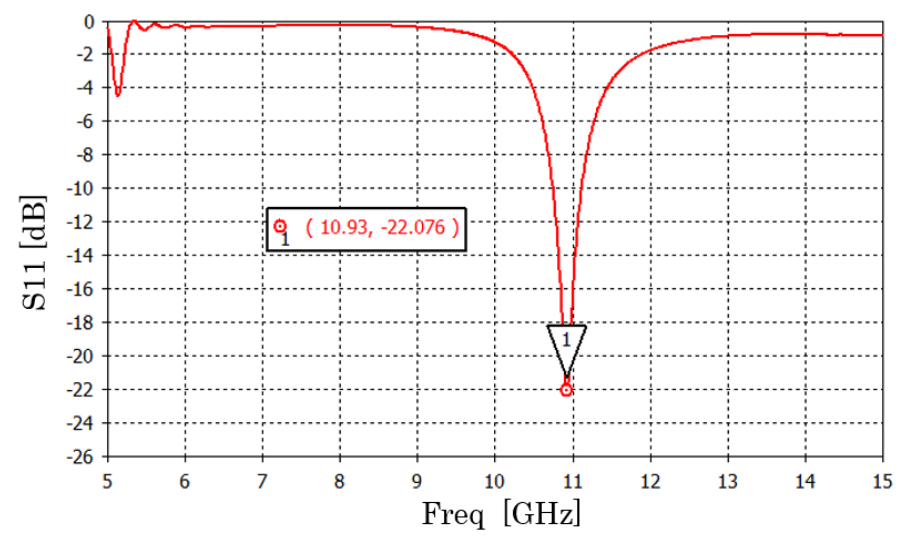

Figure IV.59: Simulated return loss of the antenna alone

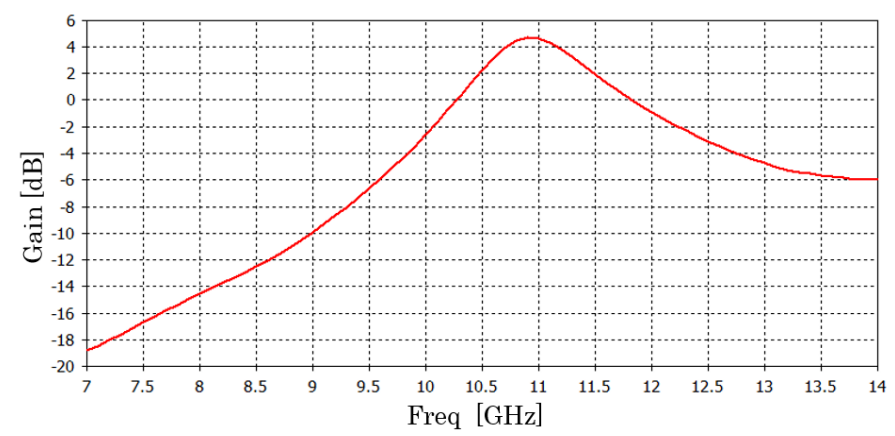

Figure IV.60 : Simulated antenna gain versus frequency 


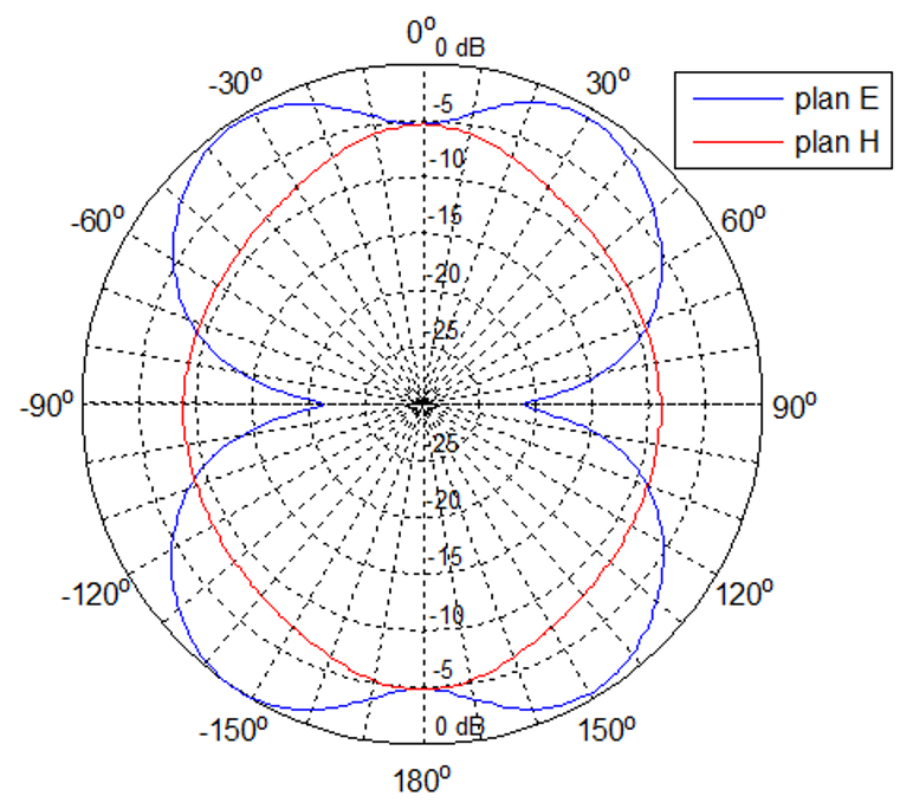

Figure IV.61 : Simulated normalized radiation pattern of the antenna at $11 \mathrm{GHz}$

Once the antenna alone was simulated and good performances were achieved, then the developed model of the W200L0.5 varactor device was integrated with the antenna to realize the matching reconfigurability operation. The simulated return loss of the AIA under various DC bias from $-3.2 \mathrm{~V}$ to $-5 \mathrm{~V}$ is presented in Figure IV.62. As can be seen, the minimum and the maximum values of the simulated return loss are, respectively, $-10 \mathrm{~dB}$ and $-6 \mathrm{~dB}$ over the bandwidth from $7.53 \mathrm{GHz}$ to $7.85 \mathrm{GHz}$ corresponding to a tunability up to $4 \%$ narrow bandwidth inside. It is clearly demonstrated that the S11 is not good as for the first simulation with only the antenna itself; here the overall impedance presented by the varactor is significantly impacting the matching of the antenna. As said before this antenna was designed and optimized to be integrated with a GaAs varactor diode. Because the antenna was already fabricated and prototypes were available in the laboratory (IETR), we kept the same design and only integrate the model of $\mathrm{GaN}$ varactor and the simulations were relunched. This is why we could not re-optimize the antenna design to achieve a good matching once the GaN varactor is integrated. However, the idea here was to validate the developed $\mathrm{GaN}$ varactor model and demonstrate the use of $\mathrm{GaN}$ varactor for reconfigurable AIA. This can be seen from the simulated S11 results where the 
resonant frequency of the antenna is electronically controlled by the DC voltage applied over the varactor model.

The structure of this used antenna is discussed in [147] where the design procedures, fabrication steps and details are presented. Maximum return loss occurred when the GaN varactor presents a high $\mathrm{R}_{\mathrm{Eq}}$ at some bias DC as plotted in chapter III.

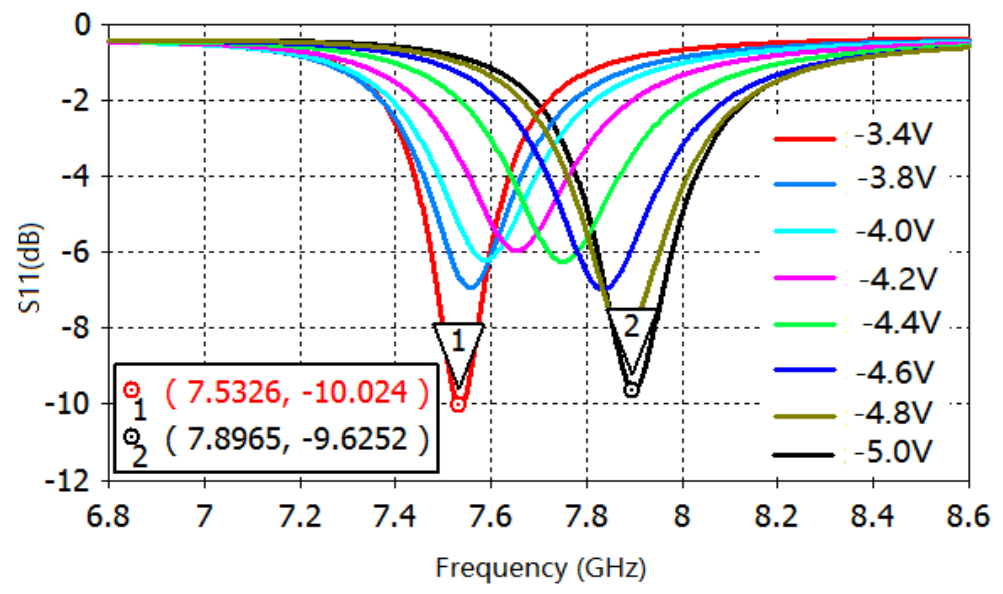

Figure IV.62: Simulated return loss of the loaded antenna for different reverse-bias voltages

\subsection{Measured Return Loss and Radiation Pattern}

In this section, we provide experimental results of the proposed active integrated antenna. In order to verify the tunability of the antenna experimentally, the GaN varactor was integrated with the antenna using the wire banding technique and then the whole design was measured.

Firstly, the measured return loss was measured and it can be seen from Figure IV.63, the $\mathrm{S} 11$ varies from $-7.13 \mathrm{~dB}$ to $-6.9 \mathrm{~dB}$ in the band from $7.53 \mathrm{GHz}$ to $7.83 \mathrm{GHz}$ which corresponds to the same simulated tunable bandwidth. Therefore, as can be seen from both Figure IV.62 and Figure IV.63, the simulation results match quite well with the actual prototype measurement even though the minimum return loss has been decreased slightly from the simulation results that can be probably 
caused by the effects of wires-bonding that connecting the GaN varactor terminals to the antenna and some parasitic resistance and inductance that is not captured in the whole equivalent proposed model. Then, in an anechoic chamber, the gain and the normalized radiation pattern of the fabricated AIA were measured in the E-plane and H-plane for several DC bias and results are, respectively, depicted in Figure IV.65 and Figure IV.66. Both E-plane and H-plane are normalized to the maximum gain value obtained at bias point of $-6 \mathrm{~V}$. The maximum beam direction occurs around $-6^{\circ}$ for the H-plane as well as for E-plane. However no diffraction in E-plane is observed unlike to what it was observed in simulations. It can be seen that in both planes, the fabricated prototype antenna generates a low cross-polarization level in the mainlobe direction about $-20 \mathrm{~dB}$ and $-25 \mathrm{~dB}$. The gain has been measured under various external applied DC bias voltages, and results are depicted in Figure IV.64. The antenna exhibits a very poor gain values from $-6.5 \mathrm{~dB}$ to $-4.7 \mathrm{~dB}$ at the frequency range from $7.55 \mathrm{GHz}$ to $7.9 \mathrm{GHz}$. The gain value decreases as the antenna resonant frequency becomes lower, which is due to the electrically downsizing effects of the antenna [148]. In addition, the relative higher $R_{\mathrm{Eq}}$ values of the GaN varactor contributes to reduce the gain values. This $\mathrm{R}_{\mathrm{Eq}}$ was discussed before in chapter III.

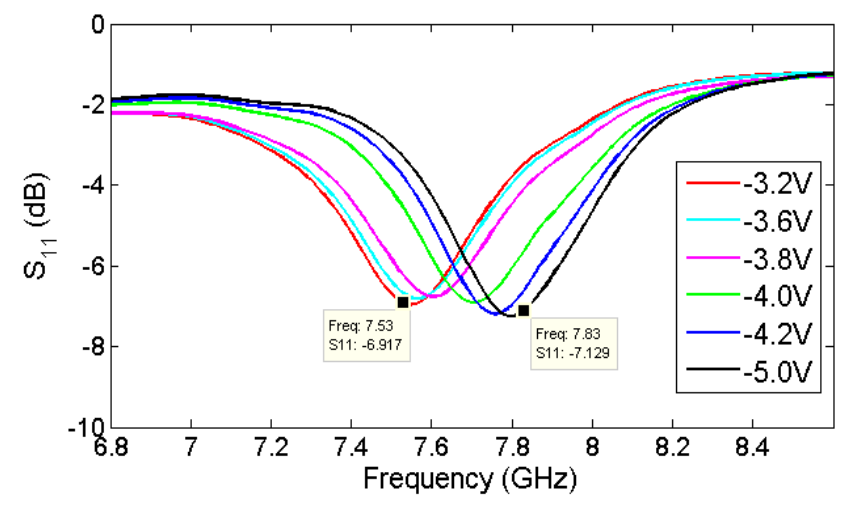

Figure IV.63: Measured return loss of the loaded antenna for different reverse-bias voltages 


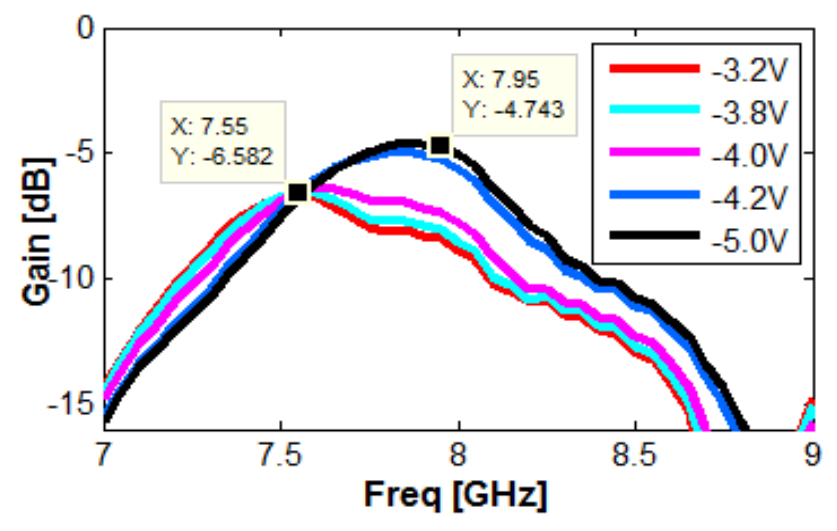

Figure IV.64: Measured loaded antenna gain versus frequency for various DC bias

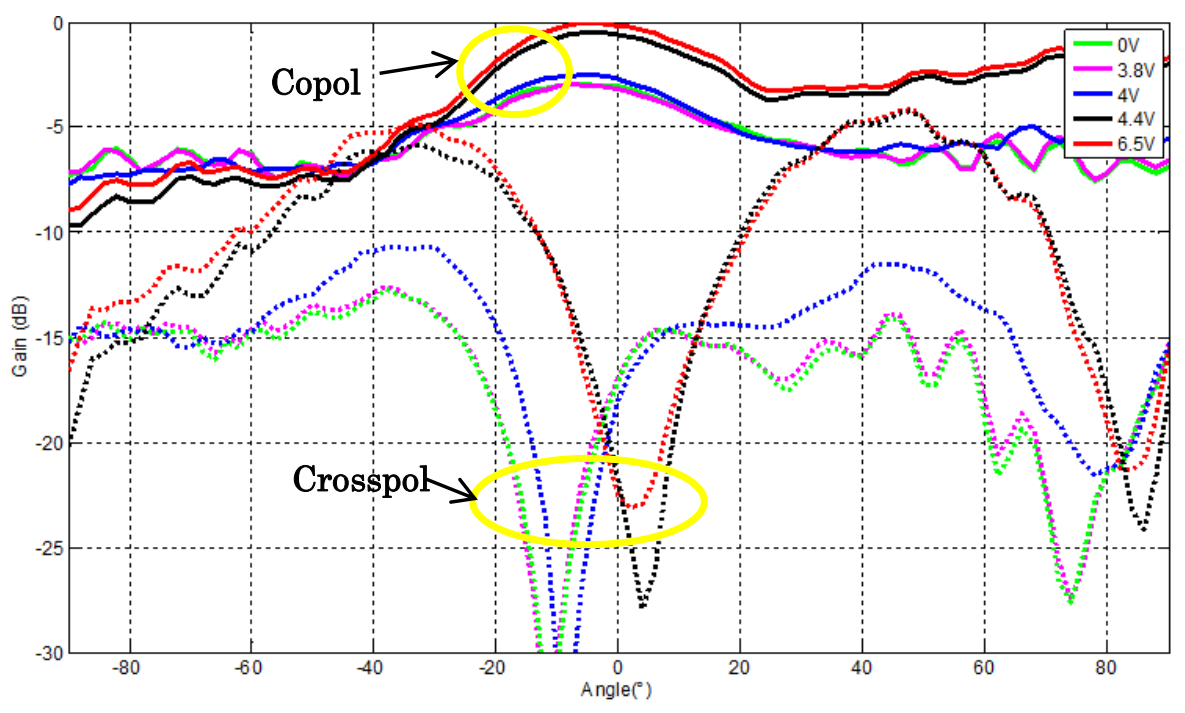

Figure IV.65 : Measured radiation pattern in H-plane of the loaded antenna under various $D C$ bias 


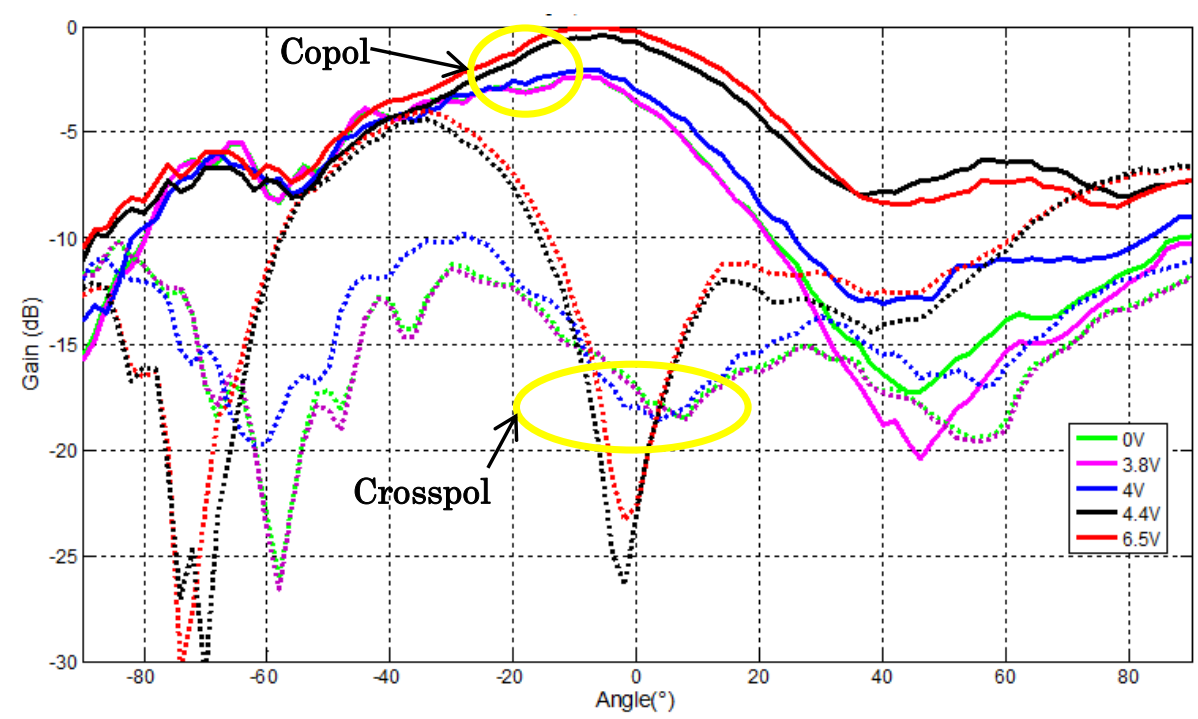

Figure IV.66 : Measured radiation pattern in E-plane of the loaded antenna under various $D C$ bias

The performance of GaN varactor has limitations for the antenna radiation efficiency due to its high $R_{E q}$ values, which is quite difficult to avoid. In addition, bandwidth is the limiting factor in using GaN varactor because of its quick variation of $\mathrm{C}_{\mathrm{Eq}}$ over a limited DC bias range as discussed in chapter III and shown in figures $\mathrm{C}_{\mathrm{Eq}}$ versus DC. Also because, we could not re-optimize the antenna design to achieve good matching over the tuning range of the varactor and good antenna gain performances, another antenna structure will be proposed to improve the performances and demonstrate the use of GaN varactor in reconfigurable matching antenna. This structure of antenna will be presented and discussed in the next section. 


\section{Reconfigurable Aperture Coupled Slot Antenna using GaN Varactor}

\subsection{Antenna Design}

In order to improve the previous proposed antenna gain and to validate the use of the developed GaN varactor diodes, another structure based on aperture coupled microstrip patch antenna feed technique is proposed. The structure of the aperture-coupled microstrip patch antenna is depicted in Figure IV.67. The antenna structure with overall dimensions $\mathrm{L}$ of $50 \mathrm{~mm}$ and $\mathrm{W}$ of $50 \mathrm{~mm}$ consists of two dielectric layers: a slot layer and a simple element patch layer and between them an air-gap layer of $1 \mathrm{~mm}$. This air-gap is obtained by using a Rohacell foam with a thickness of $1 \mathrm{~mm}$ and a relative permittivity of 1 . The square microstrip patch size is $13 \times 13 \mathrm{~mm}^{2}$ is placed above the top surface of the coplanar waveguide (CPW) slot layer with relative permittivity $\mathcal{E}$ r_p of 2.2 and thickness Tsub_p of $0.8 \mathrm{~mm}$. This means, the bottom surface of the slot layer is without metallization. The ground plane of the microstrip patch is created by the top metallization of the slot layer which is a CPW, thus resulting to improve the overall gain. The microstrip patch is fed by the slot fabricated on the bottom substrate with relative permittivity $\varepsilon_{-} \_s$ of 6.15 of and thickness Tsub_s of $0.64 \mathrm{~mm}$. The slot width is $0.3 \mathrm{~mm}$ and the slot length is $10 \mathrm{~mm}$. In order to simplify the understanding the geometry of the antenna, Figure IV.68 illustrates the stack up of the different layers. The patch is placed symmetrically to the center of the slot.

To achieve tunable frequency operation, the developed GaN-based varactor (W2000L0.5) is used and placed in the middle of the slot as zoomed and depicted in Figure IV.67. Here, the idea here is again to verify the developed GaN varactor by integrating them with a simple frequency antenna design and to design and predict the performance of reconfigurable antennas with the embedded GaN varactor. 


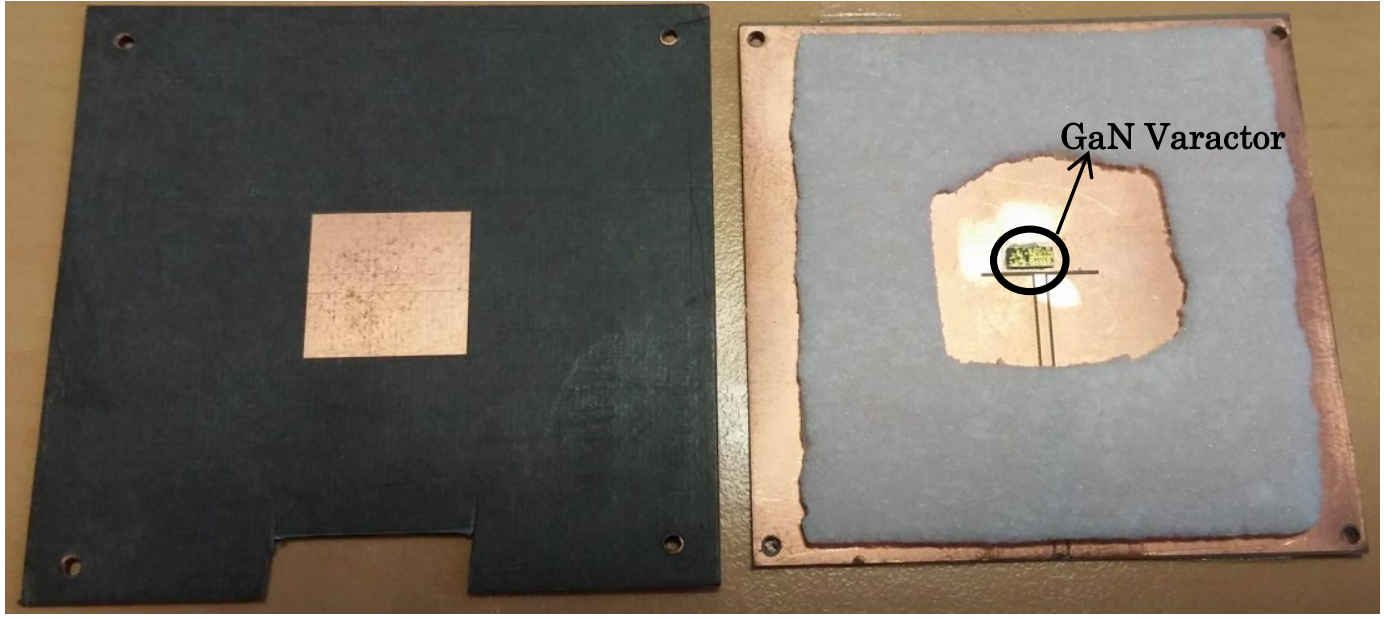

Figure IV.67 : (left) patch antenna (right) slot feeding loaded with GaN varactor

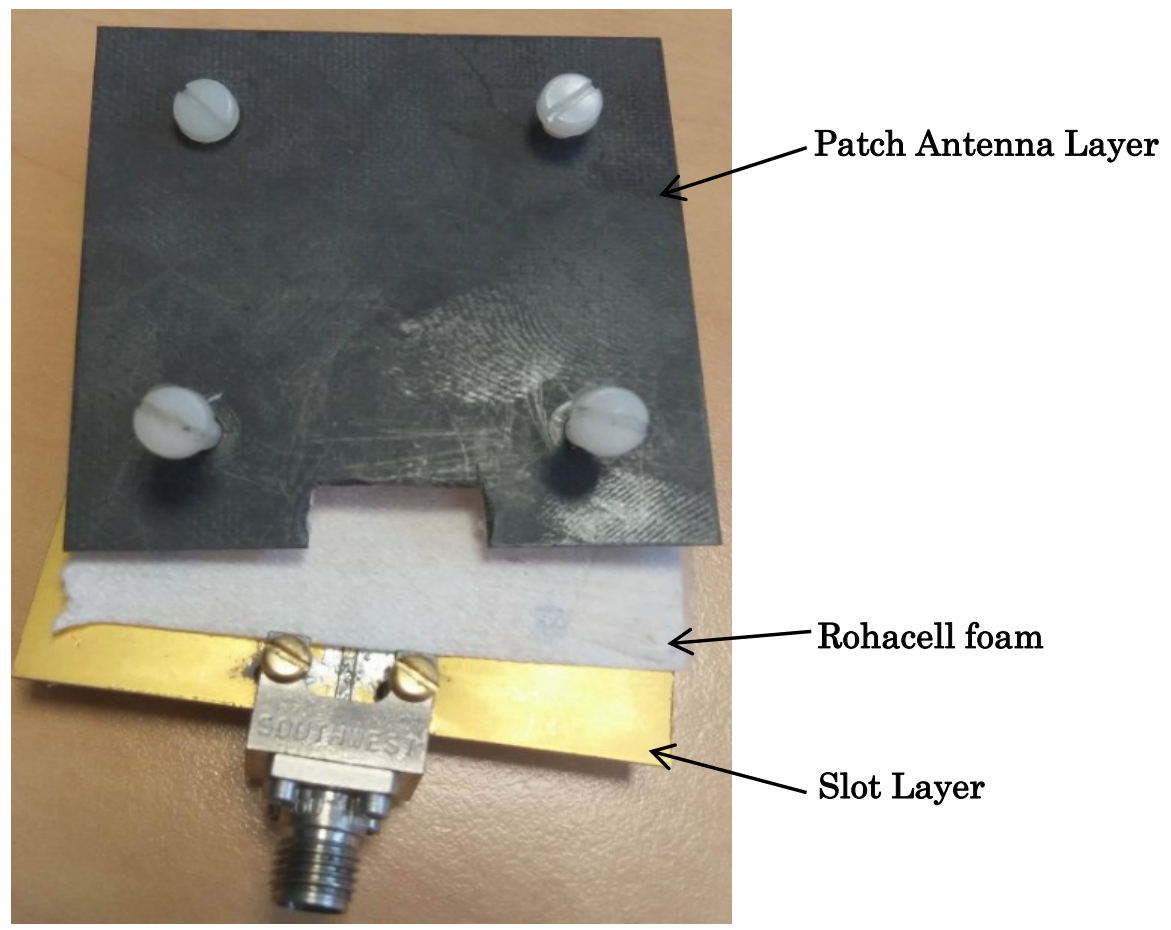

Figure IV.68 : Stuck-up whole antenna system structure

\subsection{Simulated Return Loss and Radiation Pattern}

The planar antenna is firstly simulated without integrating the developed varactor diode model. By this way, the simulated results related to the antenna itself are presented. As is shown in Figure IV.69, the simulated return loss of the antenna 
is around $-5.65 \mathrm{~dB}$ at $11.35 \mathrm{GHz}$. Once the varactor model will be added, the return loss of the antenna will be more improved and resulting to a good matching of the antenna. The maximum gain of the antenna is around $7.1 \mathrm{~dB}$ at the frequency of 11.35 GHz as seen in Figure IV.70. This is an ordinary gain value of a single patch antenna. In Figure IV.71, the normalized radiation pattern (co-polarization) of the patch antenna is simulated in $\mathrm{H}-$ plane and E-plane at $11.35 \mathrm{GHz}$ and is presented; it corresponds to a classical radiation pattern of a microstrip patch antenna in air. A very low cross-polarization level in the main lobe direction is simulated which is about $-30 \mathrm{~dB}$ in both the E-plane and in the H-plane while a back lobe level of - $12 \mathrm{~dB}$ is achieved.

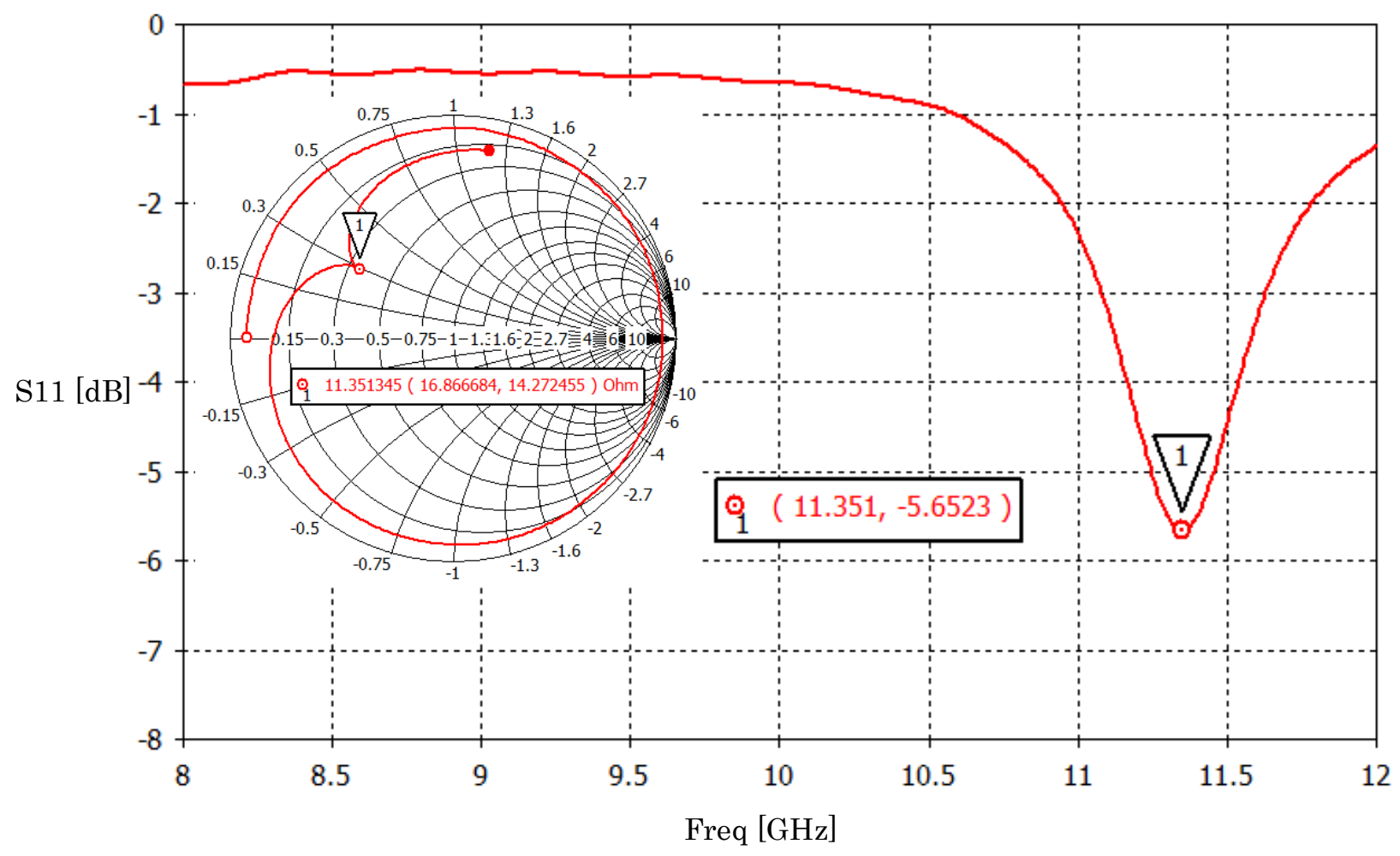

Figure IV.69: Simulated return loss of the antenna alone 


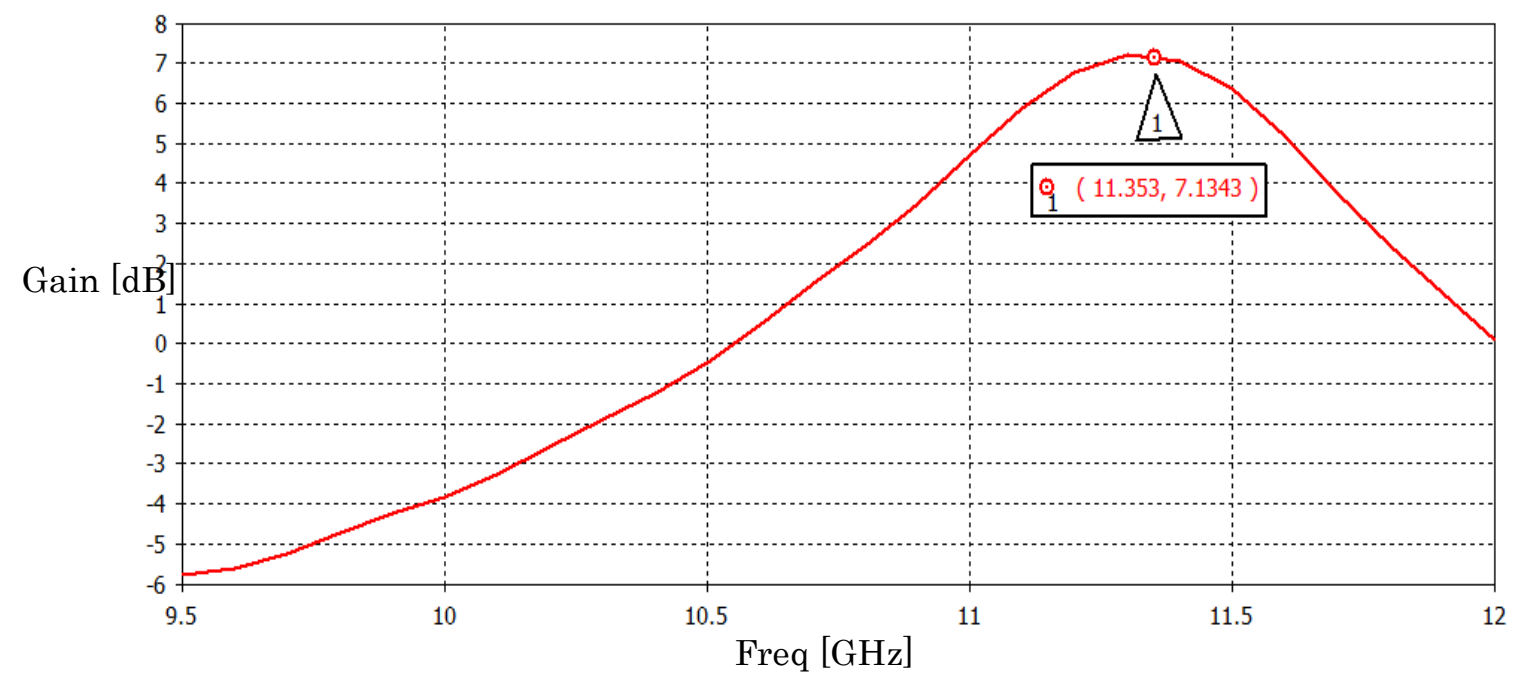

Figure IV.70 : Simulated antenna gain versus frequency

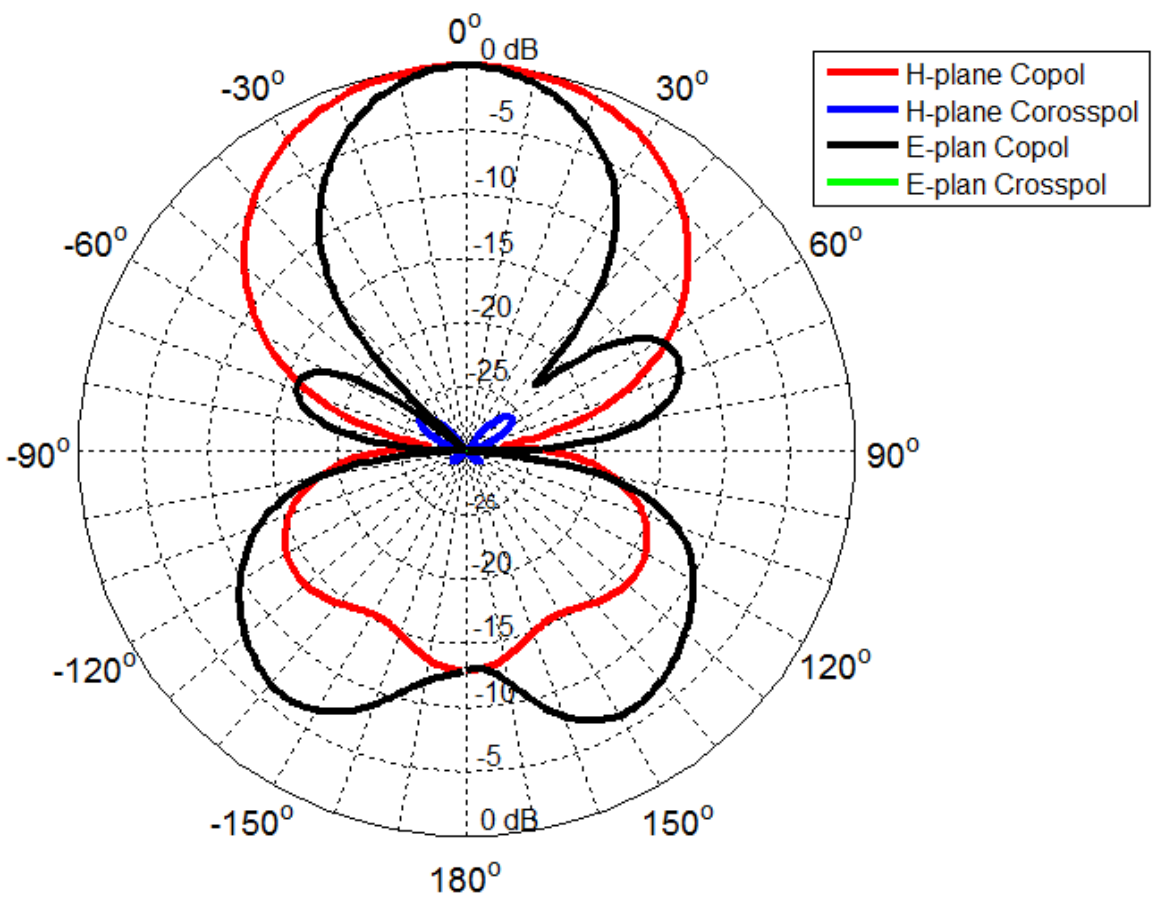

Figure IV.71 : Simulated normalized radiation pattern of the antenna at $11.35 \mathrm{GHz}$ 
The developed varactor diode was integrated with the antenna design, and the whole active antenna system was simulated. Figure IV.72 presents the variation of the return loss of the antenna versus frequency for different values of the varactor diode bias voltage. As can be seen, the minimum and the maximum values of the simulated input return loss are, respectively, $-46 \mathrm{~dB}$ and $-11.4 \mathrm{~dB}$ over a narrow bandwidth from $9.34 \mathrm{GHz}$ to $9.5 \mathrm{GHz}$. Also it can be observed that, once the varactor model was added, the antenna achieves a good matching for all the DC bias voltage better than the simulated matching for the first proposed antenna (annular slot antenna). However, the tunability of the achieved bandwidth is two times narrower. It is about $1.7 \%$ of bandwidth from $9.34 \mathrm{GHz}$ to $9.5 \mathrm{GHz}$.

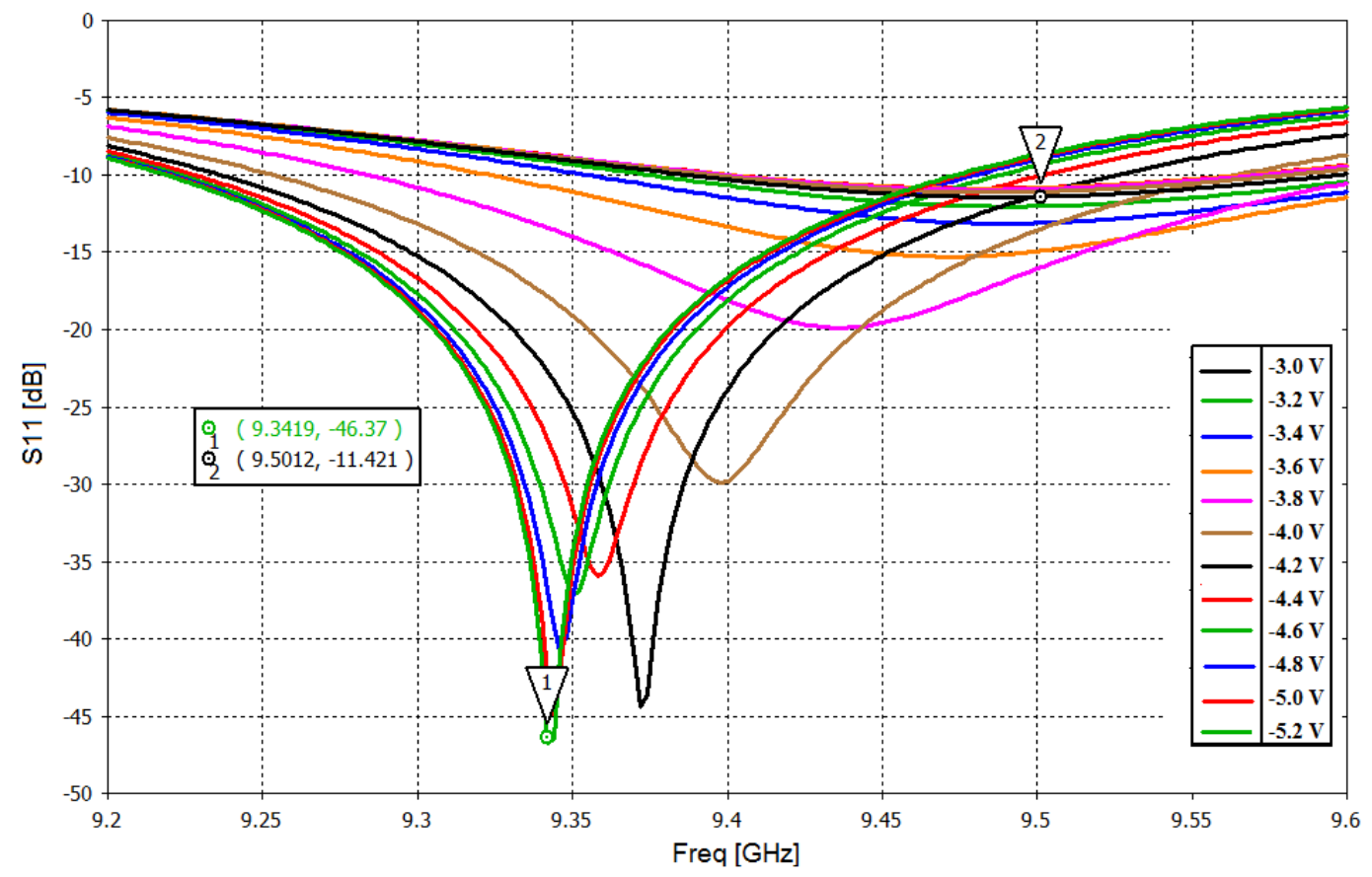

Figure IV.72: Simulated reflection coefficient (S11) of the loaded antenna for different varactor reverse-bias voltages 


\subsection{Measured Return Loss and Radiation Pattern}

To experimentally verify the simulated results of the antenna, the GaN varactor was wire-bonded over the slot layer as depicted in Figure IV.67. The first step is measure the return loss of the antenna at various DC bias value. Unfortunately, when varying the DC bias nothing change on the input reflection coefficient. We spent a lot of time to figure out where this problem comes, but we could not understand. This can be due to misconnect of the varactor with the antenna or/and the varactor device was broken during the wire-bonding steps. However, the reflection coefficient, gain and radiation pattern have been measured at $0 \mathrm{~V}$. As is shown in Figure IV.73, the measured return loss of the antenna is around $-7.3 \mathrm{~dB}$ at 10.6 GHz. Normally, at this bias point of $0 \mathrm{~V}$, as was expected with simulation, the return loss should be $-11.4 \mathrm{~dB}$ at $9.5 \mathrm{GHz}$. There a shift of almost $1 \mathrm{GHz}$, this can be due to the presence of the varactor even if it does not work as expected. The antenna gain versus frequency at this bias point is displayed in Figure IV.74. A gain value of $5.6 \mathrm{~dB}$ is achieved at $10.6 \mathrm{GHz}$, while the simulated gain value for the antenna alone at $11.35 \mathrm{GHz}$ was around $7.1 \mathrm{~dB}$. This gain difference can be due to the wires-bonding, the SMA connector used for exciting the antenna, slightly higher dielectric losses of the used substrates, and measurement uncertainty (e.g. a direction error) that were not included in the simulation. The normalized radiation pattern in the E-plane and $\mathrm{H}-$ plane at $10.6 \mathrm{GHz}$ is depicted in Figure IV.75. In both planes, the antenna array generates a cross-polarization level about $-15 \mathrm{~dB}$ in the main lobe direction which is two time larger to the simulated value. The simulated back side lobe in the main lobe direction is about $-10 \mathrm{~dB}$ which is closely corresponding to the simulated value (-12 $\mathrm{dB})$. With the measurement of the radiation pattern, no side lobes are observed unlike the simulation. This can be due to the presence in the E-plane of the connector that excites the antenna. The simulation has been done without this connector.

It was a depth disappointment that the varactor cannot work as expected to verify the simulation results. If so, this simple antenna structure would, no doubt, 
have been a good application to validate our developed GaN varactor and also to demonstrate the use of $\mathrm{GaN}$ device in reconfigurable antenna systems.

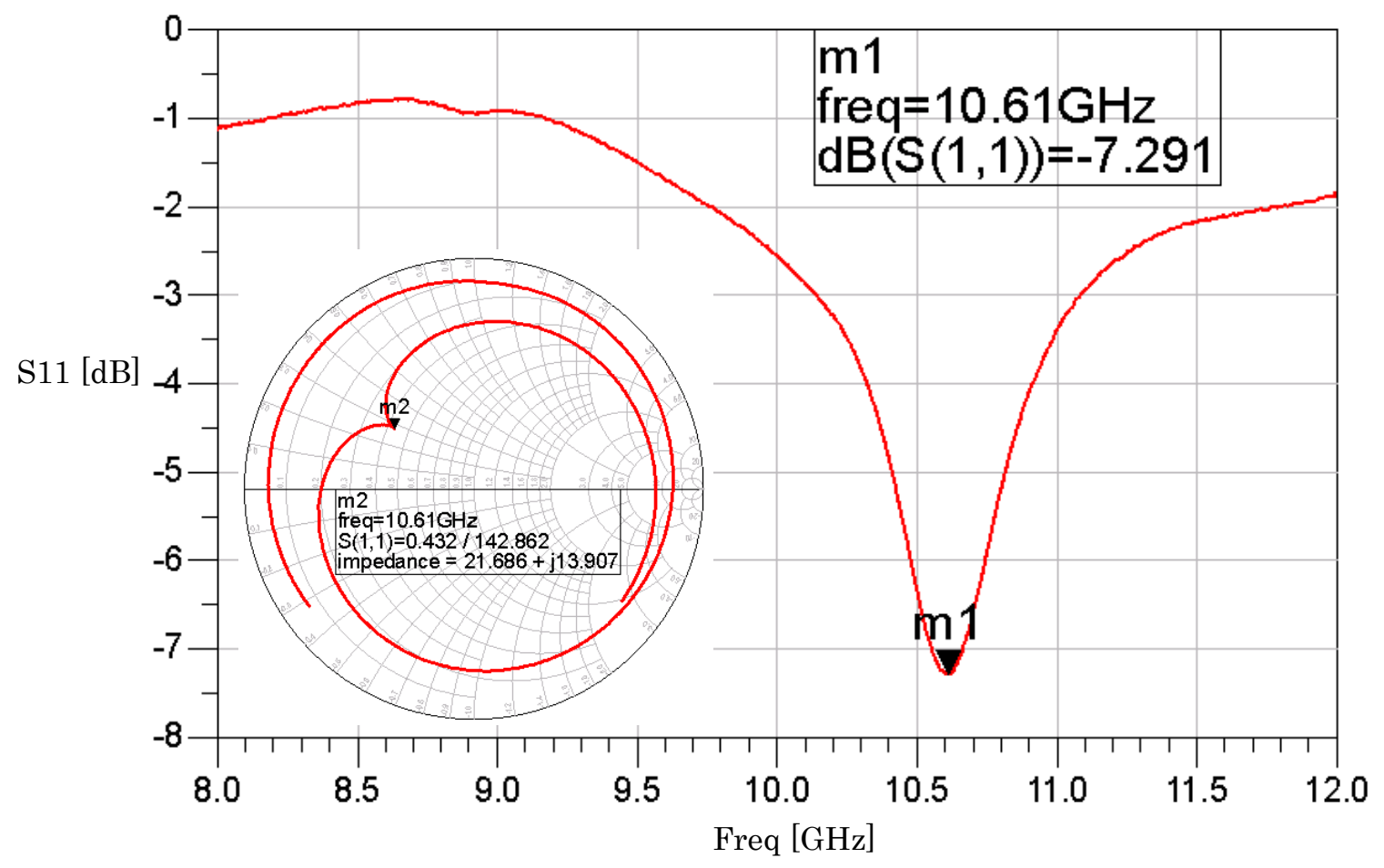

Figure IV.73: Measured return loss (S11) of the loaded antenna at no DC bias applied 


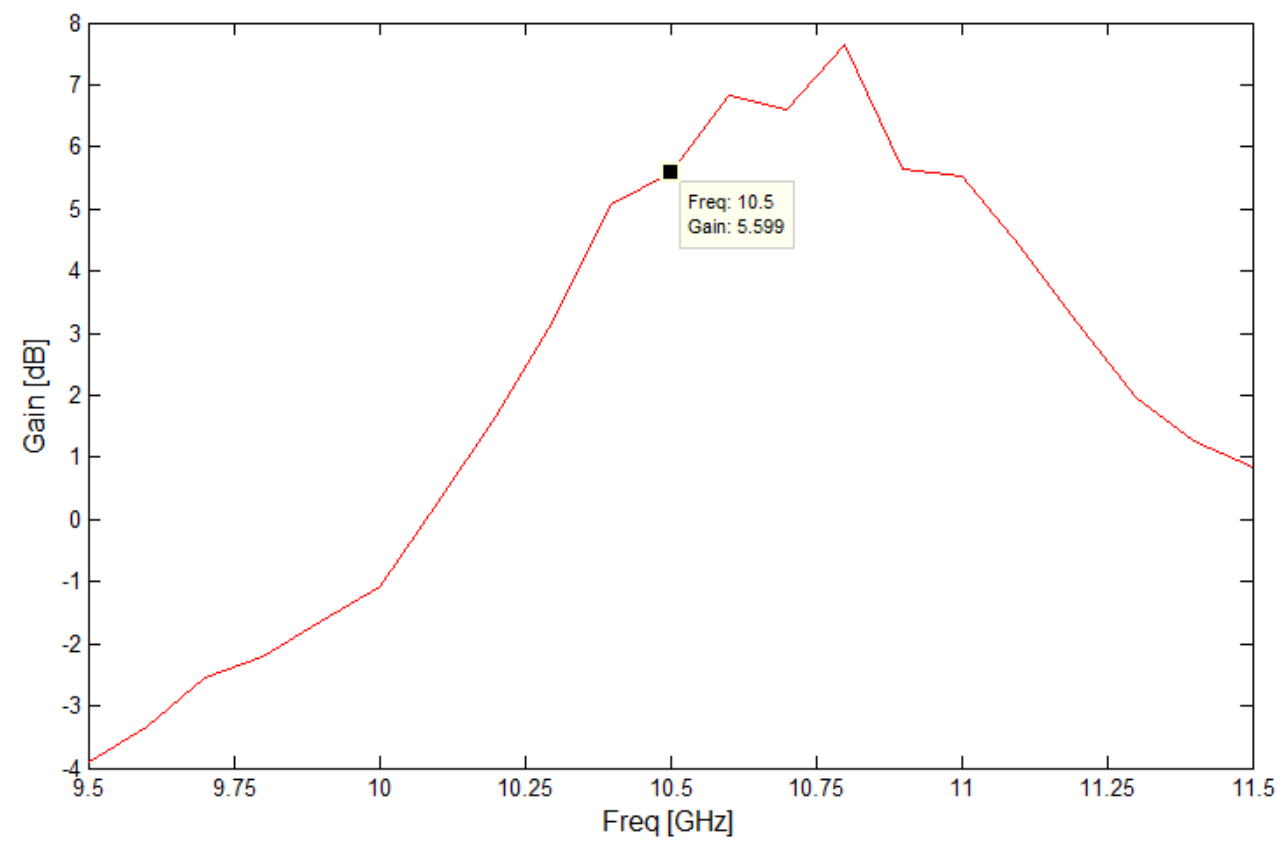

Figure IV.74: Measured loaded antenna gain versus frequency at no bias DC applied

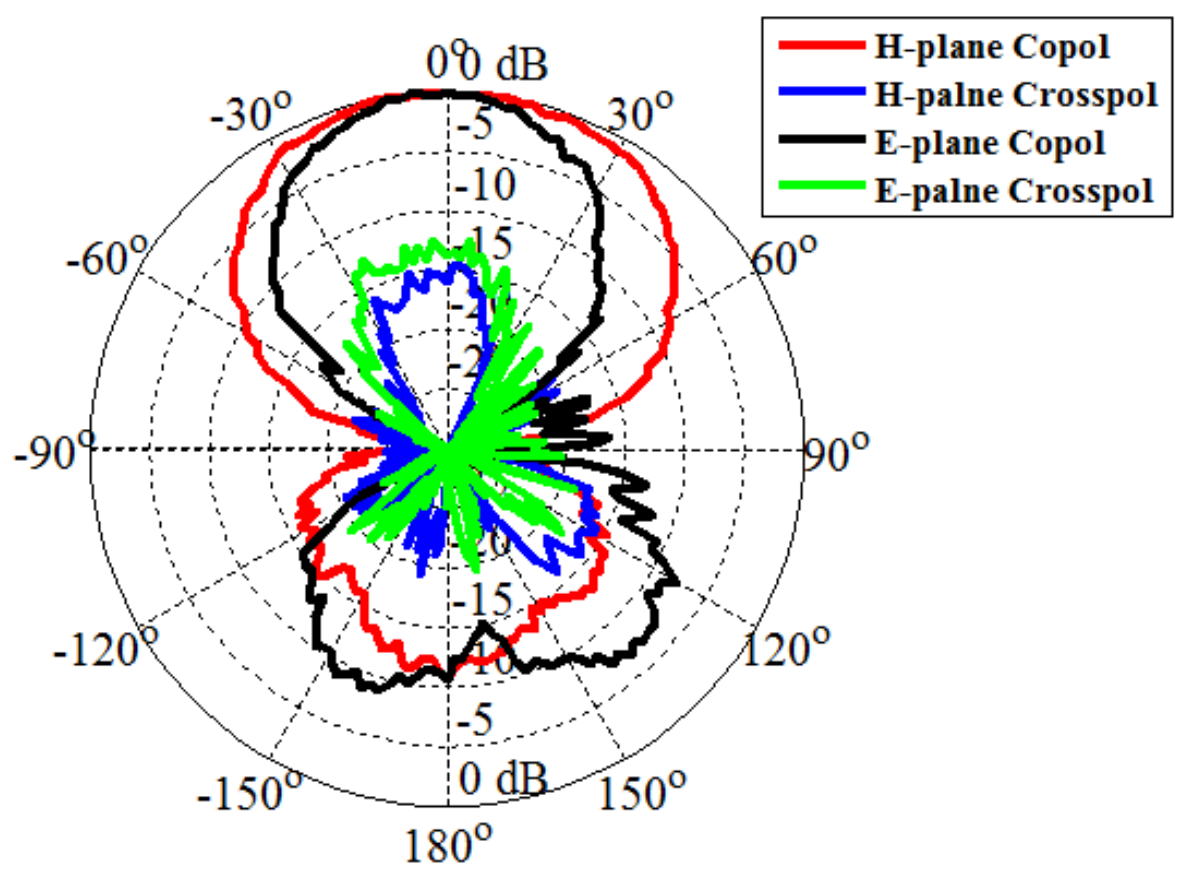

Figure IV.75:Measured normalized radiation pattern of the loaded antenna at 10.6 GHZ with no bias DC applied 


\section{Beam Steering Patch Antenna Arrays using GaN SPDT Switch Structure}

\subsection{Antenna Design}

In this section, an electronically beam steering antenna using the developed SPDT switch structure is proposed for applications that operate at 2.45 $\mathrm{GHz}$ frequency. Again, the idea here is to validate the developed GaN SPDT switch structure fabricated in NRC GaN500v2 process, for this a global system with four rectangular $4 \mathrm{x} 1$ patch array antenna is designed to achieve a beam steering over the $\mathrm{H}$-plane for four $\Phi$ possibilities such as, $0^{\circ}, 90^{\circ}, 180^{\circ}$ and $270^{\circ}$. With the use of this structure, the global array antenna can steer its beam in H-plane by a single control DC voltage (Vc_1, Vc_2, Vc_3 and Vc_4) applied at the different single switches constituting the SPDT structure. In E-plane the beam is stable for all the SPDT switch structures possibilities. As discussed in the section about SPDT switch structure, the RF performances of this switch structure degrade versus frequency, so at this frequency of $2.45 \mathrm{GHz}$, an isolation better than $-25 \mathrm{~dB}$ and an insertion loss around $-3 \mathrm{~dB}$ have been predicted from the measurements of the single switches. In addition, the antenna system was designed on Neltec substrate 9300 having $\mathcal{E}$ r of 3.5 and a thickness t of $1.6 \mathrm{~mm}$. Due to its high thickness, this substrate is very rigid and make the vertical system easier to be mounted. Therefore a cooling plate was cut from a piece of aluminium (Al), and is mounted on the backside (ground plane) of each $4 \mathrm{x} 1$ patch arrays antenna and of the feeding system design to transfers the heat generated by GaN switch structure and provides an infrastructure to mount the whole antenna system, the supply circuits and the RF connectors. The 3D model of the designed antenna system is illustrated in Figure IV.76. The whole concept size is $14 \times 14 \times 29 \mathrm{~cm}^{3}$. As shown in Figure IV.76, the four sides rectangular $4 \mathrm{x} 1$ patch array antennas with series feed are placed perpendicular over the feeding system as illustrated in Figure IV.77. Here 4 elements are used and each elements has the same 
dimensions with a length of $32 \mathrm{~mm}$ and a width of $38 \mathrm{~mm}$ in order to achieve higher gain that can be still higher to 0 once the $\mathrm{RF}$ signal goes through all the losses due to the SPDT (around - $6 \mathrm{~dB}$, wire-bonding and the substrate). In this design, the patch elements are connected using microstrip line with a length around half-wavelength $(\mathrm{Ag} / 2)$ in series feed and have been designed to operate around $2.45 \mathrm{GHz}$ with input impedance of $50 \Omega$. The length and the width of the each connecting microstrip line are, respectively, $37.7 \mathrm{~mm}$ and $1 \mathrm{~mm}$.

The SPDT switch structure are connected to the feeding system illustrated in Figure IV.78 by using wires-bonding technique as zoomed and depicted in Figure IV.79 while in Figure IV.80 a zoomed picture of the wires bonding is displayed to show the connected pads of the SPDT switch. Moreover, to facilitate the wire bonding connection between the feeding system design and the SPDT circuit, a gold layer over the copper metal was added as seen in Figure IV.78.

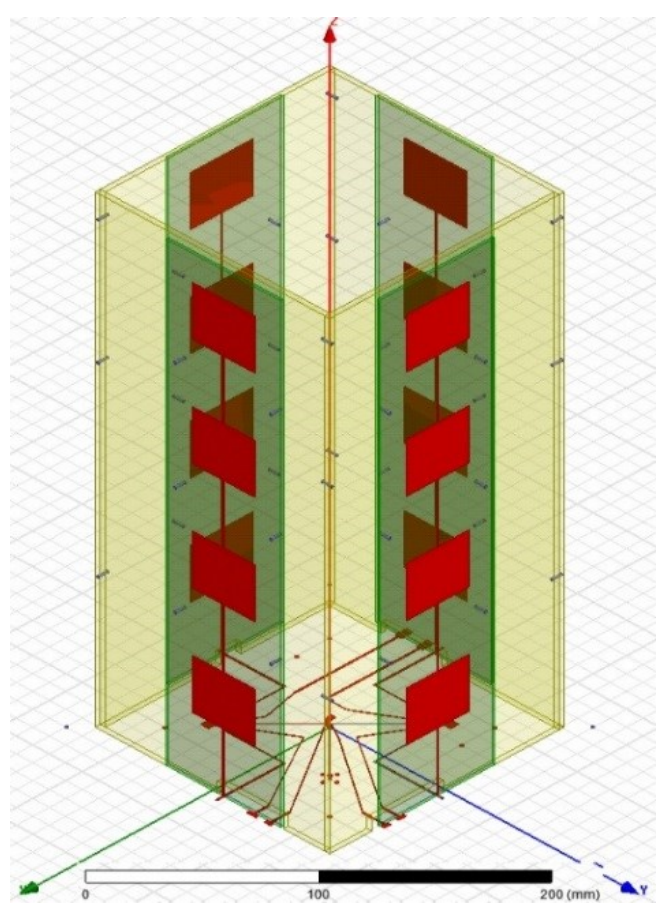

Figure IV.76: 3D model of designed antenna system. 


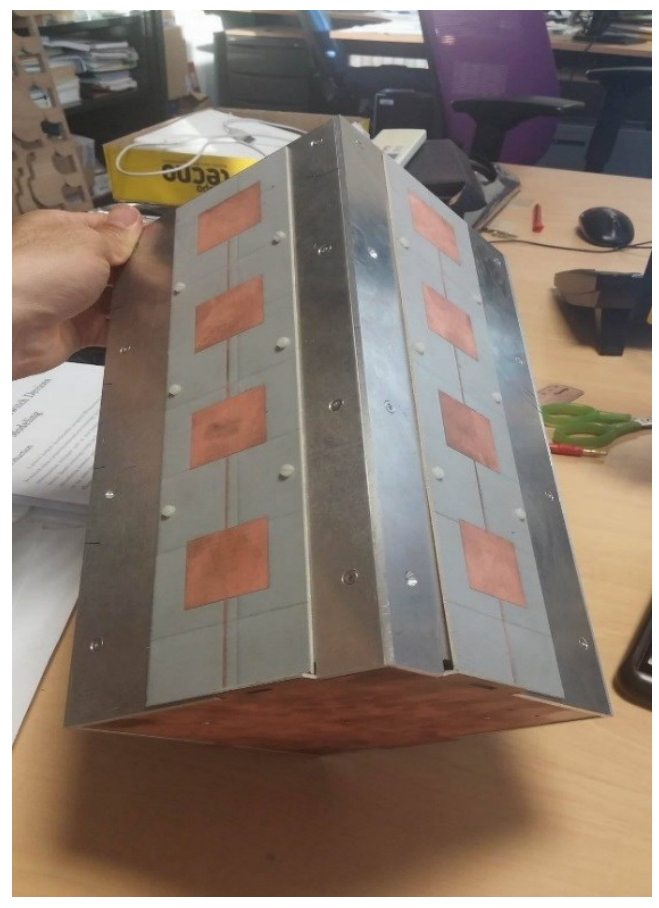

Figure IV.77: Fabricated prototype of the antenna

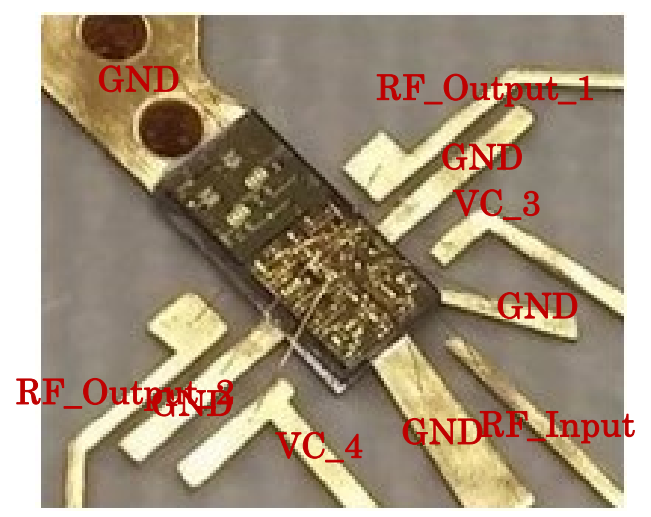

Figure IV.79: Zoom on SPDT_1 switch

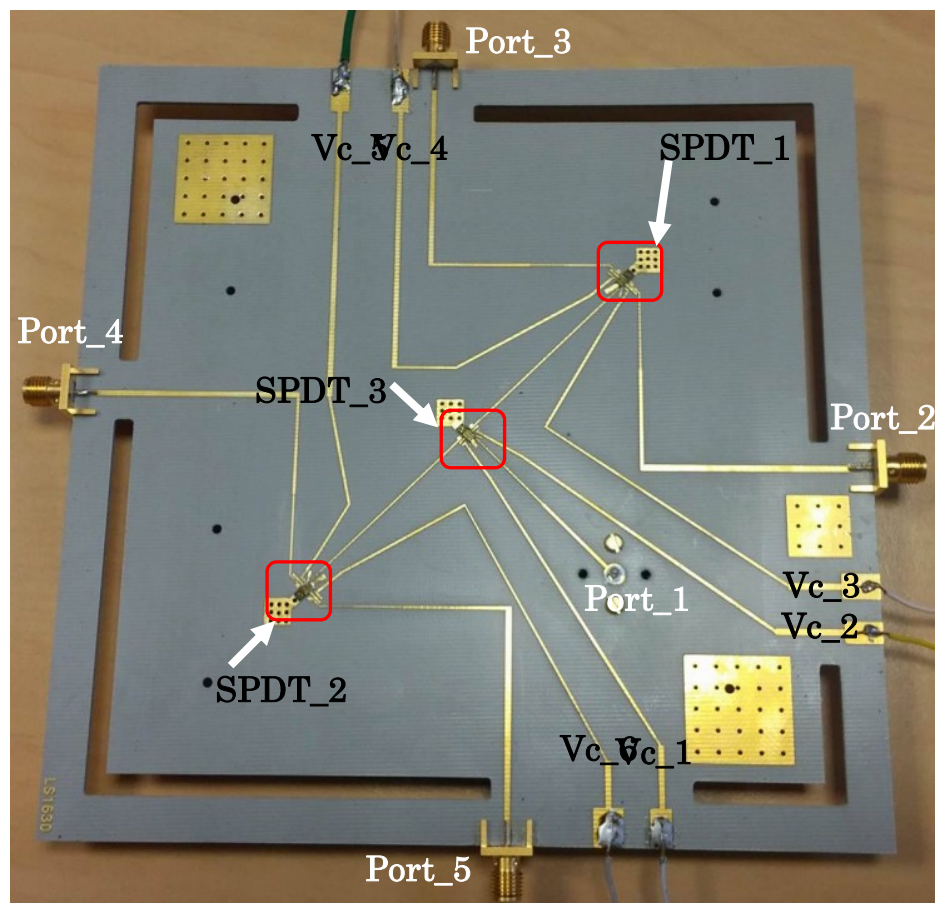

Figure IV.78: The feeding system including the three SPDT switch structures

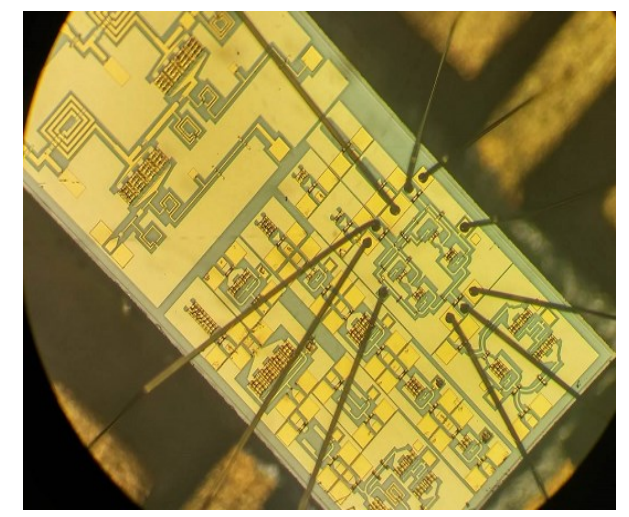

Figure IV.80: Zoom on the wires bonding connecting the SPDT_1 to The feeding system 
For a better understanding of the antenna geometry, in Figure IV.77 the fabricated final prototype is shown. One of the four $4 \times 1$ patch array antenna is depicted in the Figure IV.81. The four $4 \times 1$ patch arrays antenna are fed by four separated horizontal strips that are placed over a separated design using same Neltec substrate (see Figure IV.78) along with the bias DC control network of the SPDT switch structure. This design is called the feeding system. The four excitation strips are joined together by matching circuit including the SPDT and the $4 \times 1$ patch antenna array to $50 \Omega$. This matching circuit is based on quarter-wavelength $(\mathrm{Ag} / 4)$ matching technique. It depends of the switching states of the SPDT ports, the predicted impedances are summarized in Table IV.4. The coaxial probe is located at joint point of all the four excitation strips, which acts as antenna feed.

Table IV.4: Predicted different impedances of the SPDT ports.

\begin{tabular}{|c|c|c|}
\hline & $\begin{array}{c}\text { RF_Output_1 Port is ON } \\
\text { and } \\
\text { RF_Output_2 Port is OFF }\end{array}$ & $\begin{array}{c}\text { RF_Output_1 Port is OFF } \\
\text { and } \\
\text { RF_Output_2 Port is ON }\end{array}$ \\
\hline Impedance at RF_Input Port & $75-\mathrm{j}^{*} 20.6(\Omega)$ & $75-\mathrm{j}^{*} 20.6(\Omega)$ \\
\hline Impedance at RF_Output_1 Port & $77.05-\mathrm{j}^{*} 18.6(\Omega)$ & $60.10-\mathrm{j}^{*} 9.85(\Omega)$ \\
\hline Impedance at RF_Output_2 Port & $60.10-\mathrm{j}^{*} 9.85(\Omega)$ & $77.05-\mathrm{j} * 18.6(\Omega)$ \\
\hline
\end{tabular}

As depicted in Figure IV.78, three SPDT switches were placed between the four patch antenna arrays and the matching circuit. The first one was placed to steer the beam for $0^{\circ}$ and $90^{\circ}$, the second one to scan the beam for $180^{\circ}$ and $270^{\circ}$, while the third one was placed between the main feeding transmission line (connected to the cable coaxial) and the two first SPDT to switch the RF signal to a one of them at once. Due to unavailability of a complete model of the SPDT switch structure, as mentioned in Chapter III in SPDT switch section, depending upon the state of the SPDT switch a short circuit or open circuit was used between the corresponding patch antenna array and the matching circuit when necessary to conduct the EM simulation. 


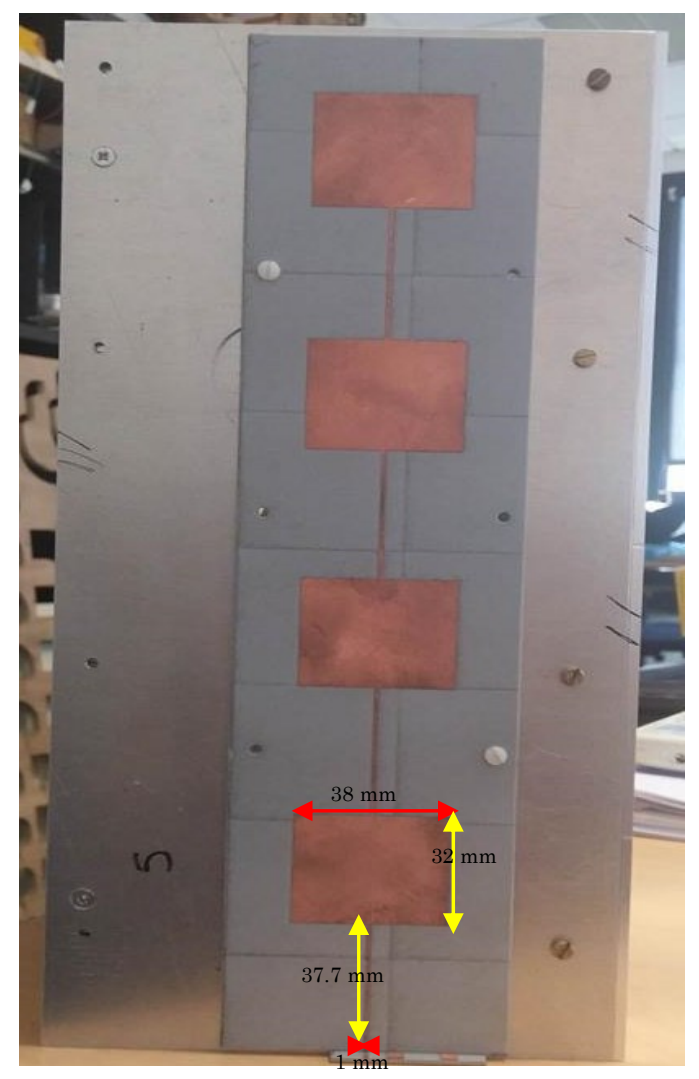

Figure IV.81: Front view of the fabricated antenna

\subsection{Simulated Return Loss and Radiation Pattern}

The EM simulations allowing to get the return loss and the radiation pattern were performed by using HFSS. Table IV.5 summarizes the four cases or the four beam steering possibilities corresponding to the three SPDT switches states.

Table IV.5: Switching configuration to achieve radiation pattern reconfigurability.

\begin{tabular}{|c|c|c|c|c|c|c|c|}
\hline & \multicolumn{2}{|c|}{ SPDT_1 } & \multicolumn{2}{c|}{ SPDT_2 } & \multicolumn{2}{c|}{ SPDT_3 } & (deg) \\
\cline { 2 - 8 } & RF_Output_1 & RF_Output_2 & RF_Output_1 & RF_Output_2 & RF_Output_1 & RF_Output_2 & \\
\hline Case_1 & ON & OFF & OFF & OFF & OFF & ON & $0^{\circ}$ \\
\hline Case_2 & OFF & ON & OFF & OFF & OFF & ON & $90^{\circ}$ \\
\hline Case_3 & OFF & OFF & ON & OFF & ON & OFF & $180^{\circ}$ \\
\hline Case_4 & OFF & OFF & OFF & ON & ON & OFF & $270^{\circ}$ \\
\hline
\end{tabular}


As highlighted in one $4 \times 1$ patch arrays antenna return loss which is shown in Figure IV.82, an impedance bandwidth of approximately $40 \mathrm{MHz}(2.45 \mathrm{GHz}$ to 2.41 $\mathrm{GHz}$ ) is achieved, with a resonance frequency sensing at approximately $2.43 \mathrm{GHz}$. At this frequency, a return loss value better than $-30 \mathrm{~dB}$ is obtained in simulation for one $4 \times 1$ patch arrays antenna. Because the return loss of the four $4 \mathrm{x} 1$ patch arrays antenna are similar, here we report only return loss for one which corresponding to one case.

The radiation pattern in $\mathrm{H}$-plane corresponding to the four cases possibilities is shown in Figure IV.84. A shift of $90^{\circ}$ between the four cases in the radiation pattern is clearly achieved. The $3-\mathrm{dB}$ beam width where the four beam configuration cases intersect for each case is found to be approximately $85^{\circ}$.

For a better understanding of beam switching, the antenna radiation pattern is tilted towards the positive $\mathrm{z}$-axis having maximum at $\Phi=90^{\circ}, \theta=0^{\circ}$, here switches are configured as mentioned in Table IV.5 for case 1, is tilled towards the positive $\mathrm{x}$-axis when switches are configured as mentioned in case 2 having maximum at $\Phi=0^{\circ}, \theta=90^{\circ}$, is tilled towards the negative $\mathrm{z}$-axis when switches are configured as mentioned in case 3 having maximum at $\Phi=90^{\circ}, \theta=-180^{\circ}$ and for the fourth case it is tilled towards the negative $\mathrm{x}$-axis when switches are configured as mentioned in case 4 having maximum at $\Phi=-180^{\circ}, \theta=-90^{\circ}$.

The overall gain of one $4 \mathrm{x} 1$ patch arrays antenna is found to be above 10.9 $\mathrm{dB}$ at the operating frequency of 2.43 as depicted in Figure IV.83. It is important to keep in mind that in the EM simulation only short and open were used instead of the model of the SPDT, this means that almost -3dB of losses of one SPDT switch structure has to be considered. So this will results on the maximum antenna gain value that would decrease with almost $-6 \mathrm{~dB}$ because the $\mathrm{RF}$ signal go through two SPDT switch structures as explained in Table IV.5 . Furthermore, there will be also other losses due to the substrate and the wires-bonding connecting the SPDT to the 
transmission lines. The overall losses is measured for every case and will be presented later in this section.

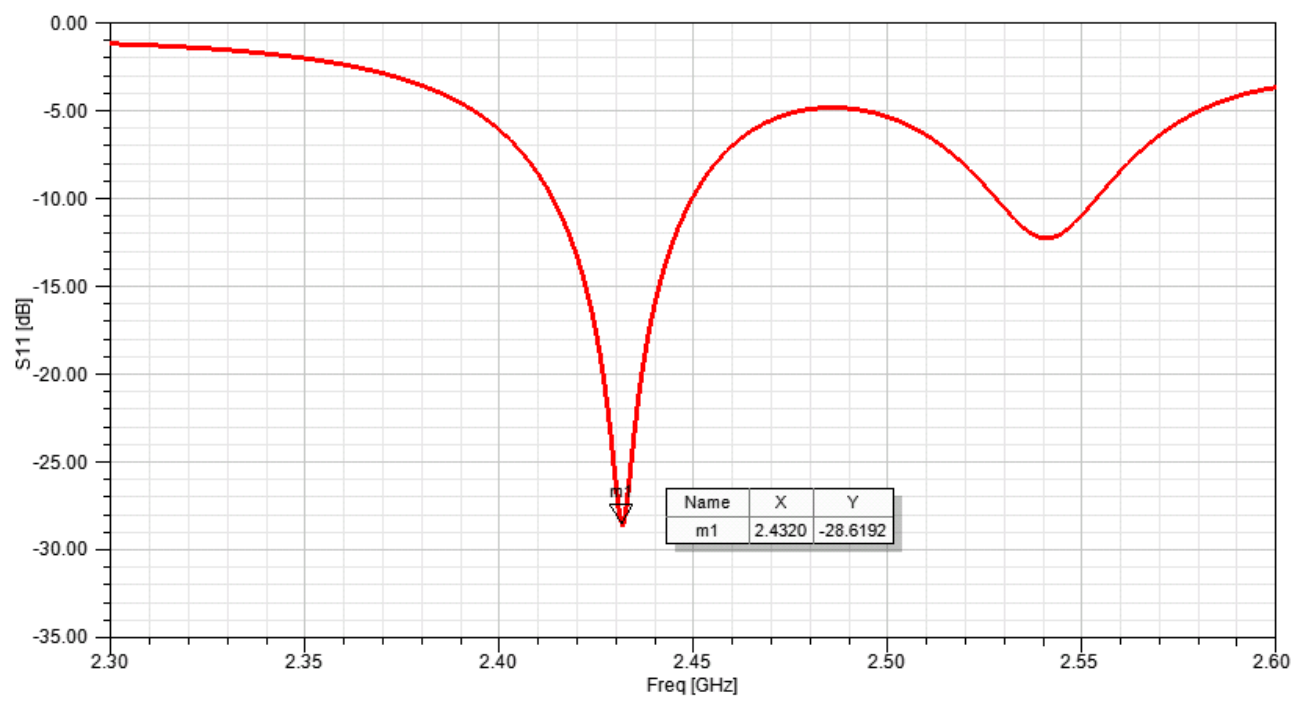

Figure IV.82: Simulated return loss of the antenna

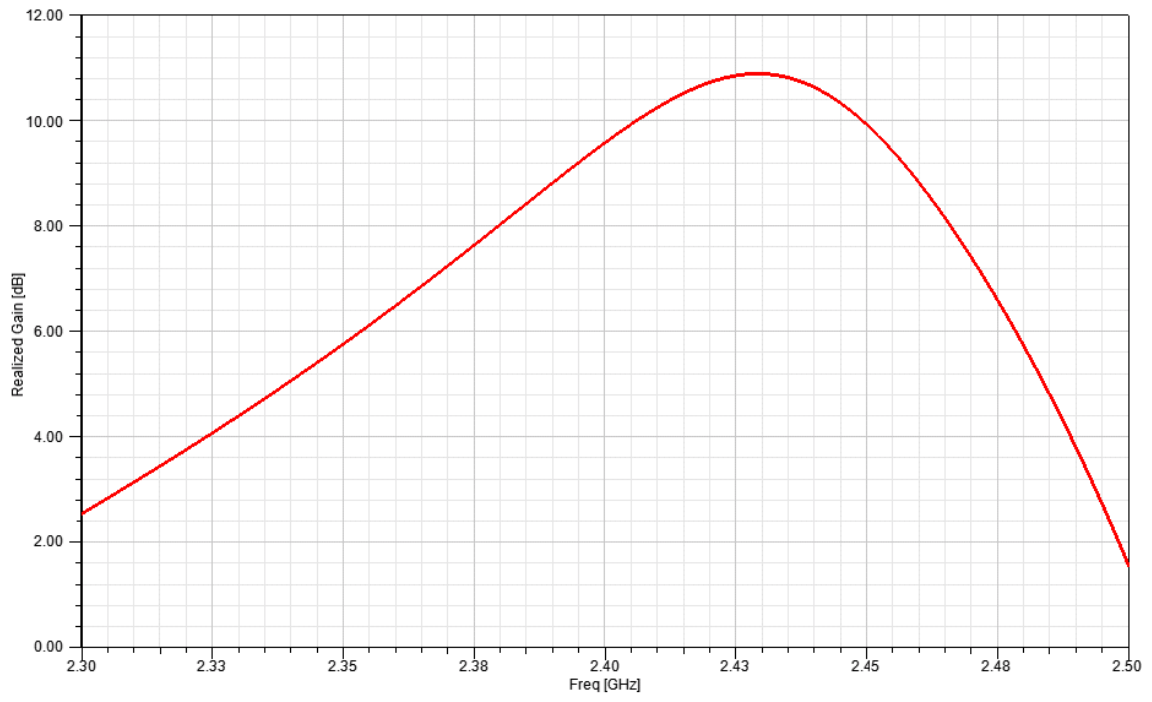

Figure IV.83: Simulated antenna gain versus frequency 
Case 1

Case 2

Case 3

Case 4

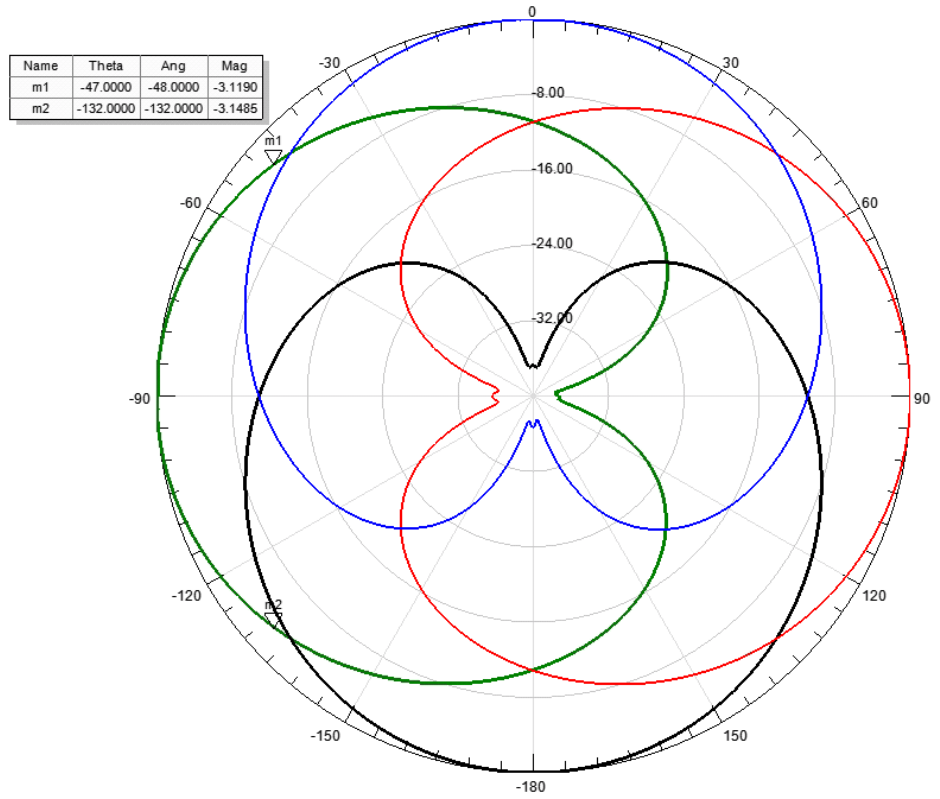

Figure IV.84: Simulated normalized $\mathrm{H}$-plane at $2.43 \mathrm{GHz}$ for four cases mentioned in Table IV.4

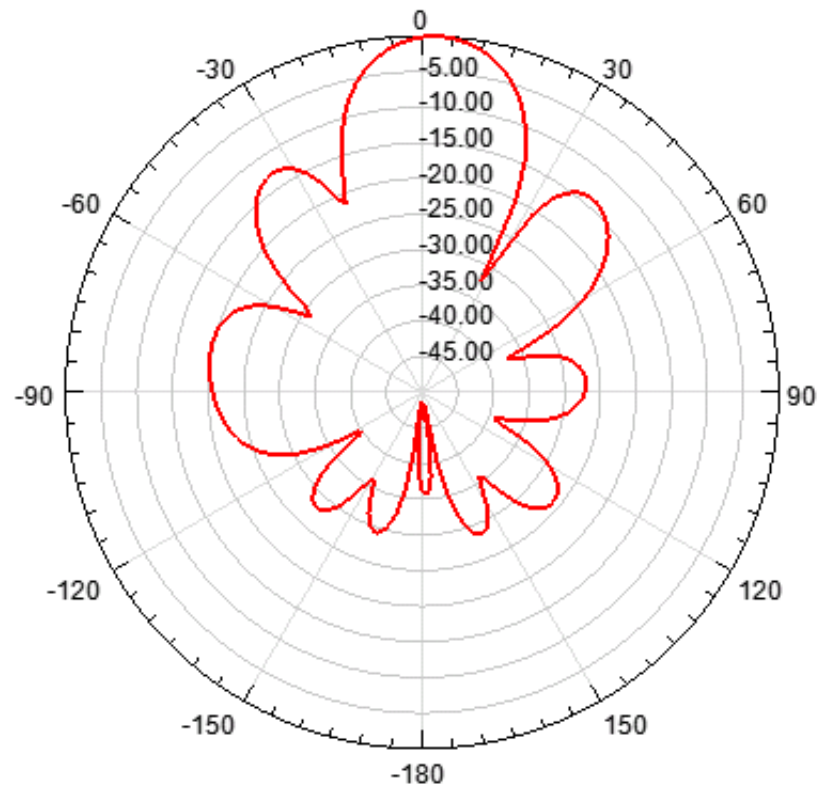

Figure IV.85: Simulated normalized E-plane at $2.43 \mathrm{GHz}$ 


\subsection{Measured Return Loss and Radiation Pattern}

Here, the experimentally measurements are presented and results are discussed. These measurements will verify the simulation results. Figure IV.86 shows the return loss of one fabricated $4 \mathrm{x} 1$ patch arrays antenna prototype. It indicates that the $4 \times 1$ patch arrays antenna presents a good match with $|\mathrm{S} 11|$ of $25.5 \mathrm{~dB}$ at a resonant frequency of $2.43 \mathrm{GHz}$ which is completely corresponding to what was achieved with simulation where a $|\mathrm{S} 11|$ of $-28.6 \mathrm{~dB}$ was obtained at 2.43 $\mathrm{GHz}$. This value of the return loss was verified for all four fabricated $4 \mathrm{x} 1$ patch arrays antenna, and here we report only return loss for one which corresponding to one case. The antenna gain versus frequency is displayed in Figure IV.87. A gain value of $10.1 \mathrm{~dB}$ is achieved at $2.43 \mathrm{GHz}$, while the simulated gain value for the antenna alone at this frequency was around $10.9 \mathrm{~dB}$. Conductor losses, slightly higher dielectric losses of the used substrate, also some set up errors during the measurement, can be the main reasons of the $0.8 \mathrm{~dB}$ gain difference. As was expected, this measured gain of $10.1 \mathrm{~dB}$ decreased by almost $-7 \mathrm{~dB}$ once the measurement with the SPDT structure is conducted. $-6 \mathrm{~dB}$ corresponding to the losses of the two SPDT structures as explained in Chapter III in Figure III.57 while the $-1 \mathrm{~dB}$ is due to the substrate losses, the transmission lines and the wire banding.

The simulated radiation pattern of the patch array antenna in both $\mathrm{H}^{-}$ plane and E-plane has been verified by measurement at $2.43 \mathrm{GHz}$. Satisfactory agreement with the simulation was achieved as shown in Figure IV.88 where the measured radiation pattern in the $\mathrm{H}$-plane for four cases is illustrated while in Figure IV.89 depicts the measured radiation pattern in the E-plane. It is seen that the beam steering operation of the radiation pattern in H-plane is achieved over the expected four direction. The radiation pattern shows the cross-pol level in the broadside direction lower than $-30 \mathrm{~dB}$ in the $\mathrm{H}$-plane and $-25 \mathrm{~dB}$ in the E-plane and a low side lobes of $-15 \mathrm{~dB}$ in the E-plane. 


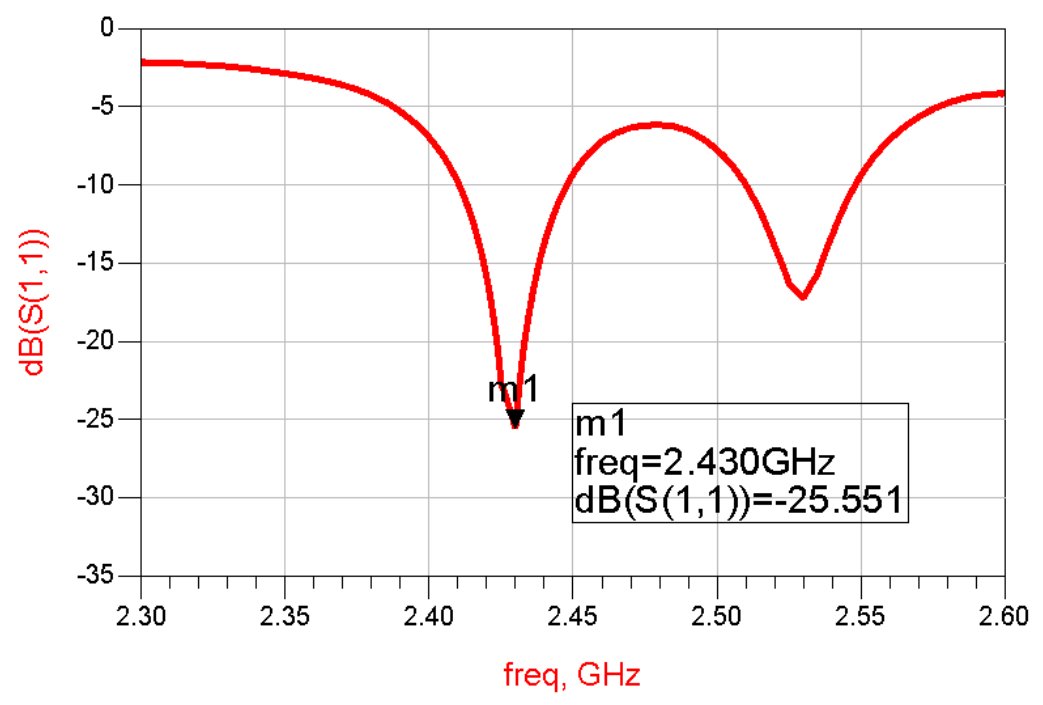

Figure IV.86: Measured return loss of the antenna

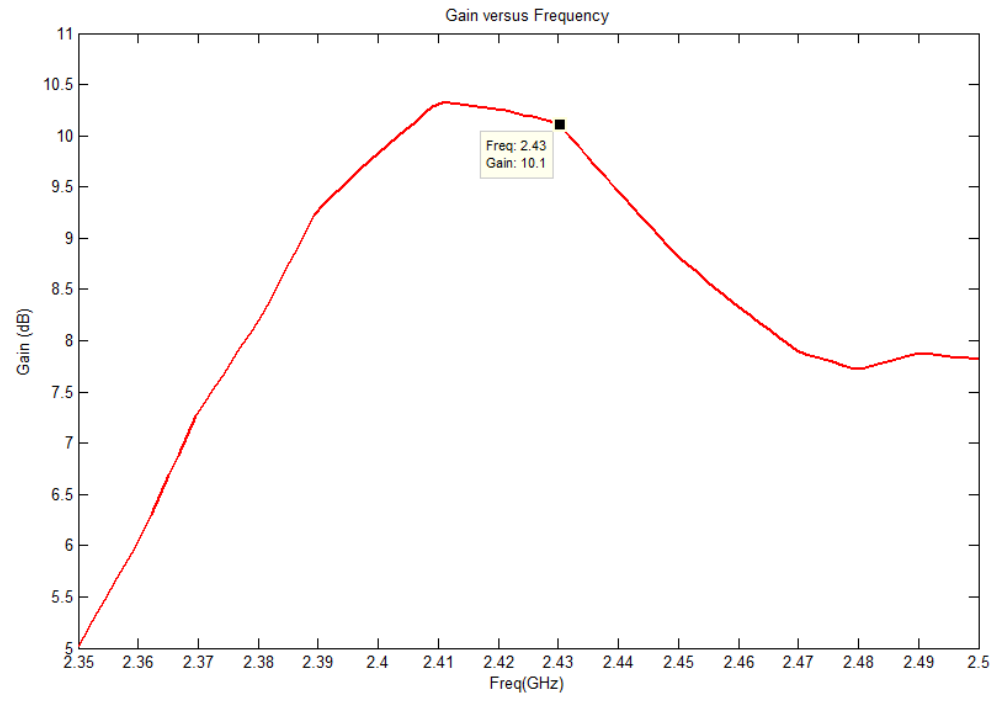

Figure IV.87: Measured antenna gain versus frequency 


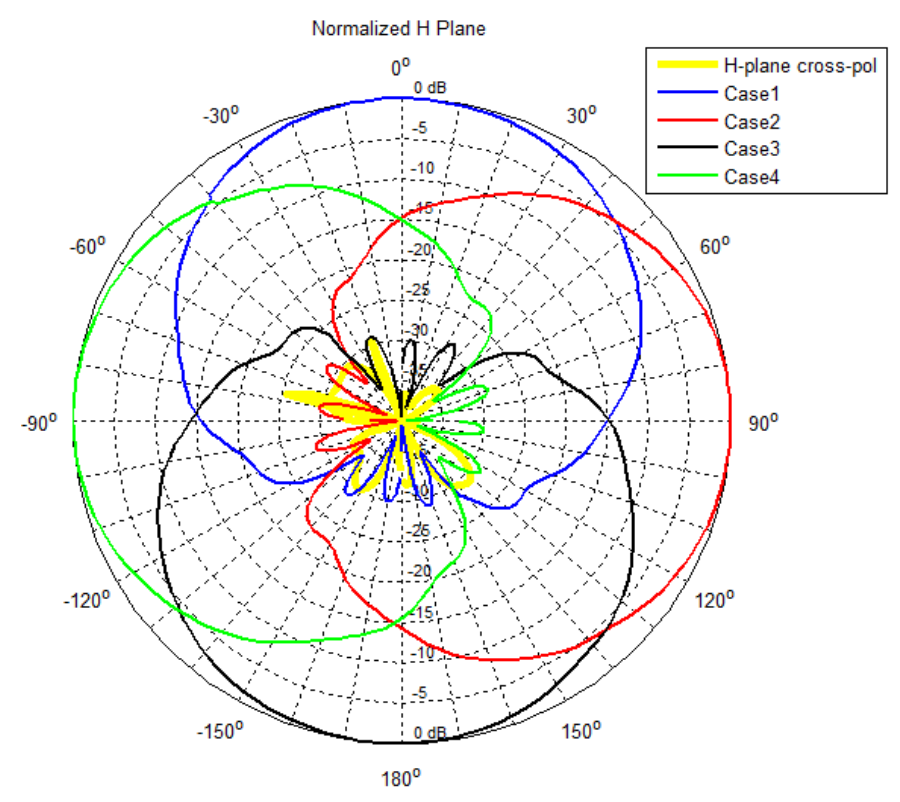

Figure IV.88: Measured normalized $\mathrm{H}$ plane at $2.43 \mathrm{GHz}$ for four cases mentioned in Table IV.4

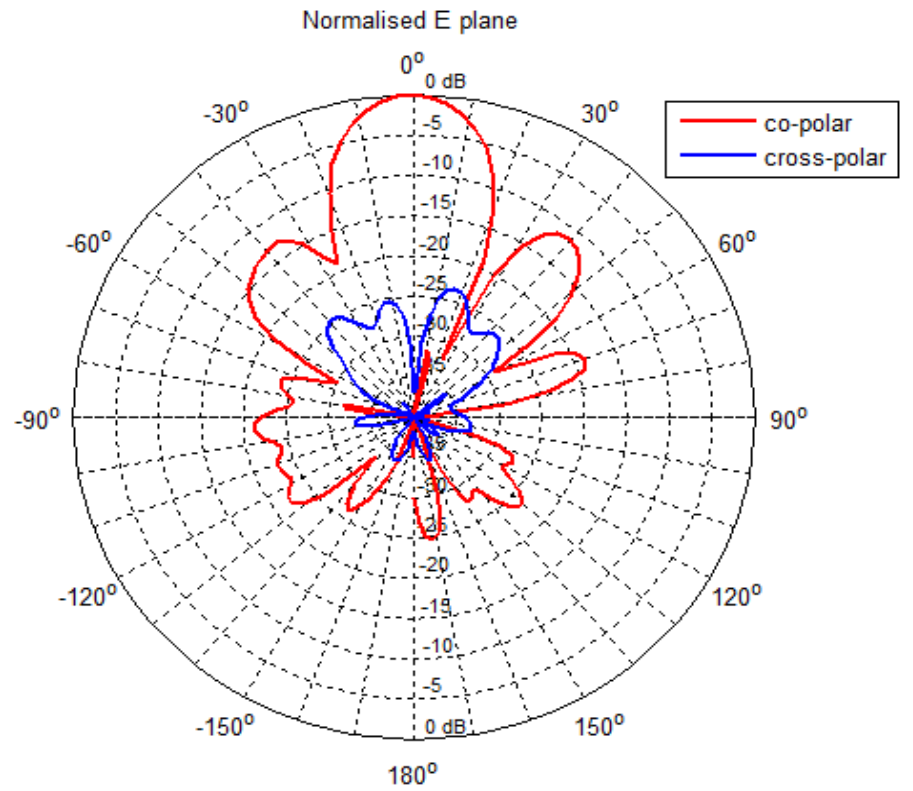

Figure IV.89: Measured normalized E plane at $2.43 \mathrm{GHz}$ 


\subsection{Measured Insertion Loss and Isolation of the Feeding System}

\subsubsection{Small Signal Measurements}

Depending on the two states of the three SPDT switch structures (as indicated in Table IV.5), the s-parameters at the different four ports, Port_1, Port_2, Port_3, Port_4 and Port_5, (see Figure IV.78) of the feeding system design have been measured. The s-parameters measurements are to be made between the common port, Port_1, and one of the others output ports, either Port_1, Port_2, Port_3 or Port_4.

When the SPDT is configured for case_1, the insertion loss is measured between the Port_1 and the Port_2 along with the isolation at the other ports. As illustrated in Figure IV.90, the insertion loss and the isolation are shown. It can be seen that the return loss at both ports (i.e Port_1 and Port_2) indicates low reflections around $-6.8 \mathrm{~dB}$ at the frequency of interest $2.43 \mathrm{GHz}$ since at this frequency the input port and the two output ports of the three SPDT have well matched to $50 \Omega$. Because the RF signal go from Port_1 to Port_2 through two SPDT switch, the measured -6.8 $\mathrm{dB}$ insertion loss value is due to the insertion loss of SPDT switches which was around $-3 \mathrm{~dB}$ as would be expected for one SPDT and the losses of the used substrate, the metal and the wires bond which can be $-0.8 \mathrm{~dB}$. The measured isolation is higher than $-25 \mathrm{~dB}$ at all ports and all configurations cases.

Similarly, the same considerations of measurement are applied if the measurements are between the Port_1 and the other output ports. In Figure IV.91, Figure IV.92 and Figure IV.93, the measured insertion loss and the isolation are displayed, respectively, for case_2, case_3 and case_4. It is clearly seen that, depending the case, measured the insertion loss is around -7.14 dB (i.e case_2) at the worst value and is around $-6.62 \mathrm{~dB}$ (i.e case_4) at the good value. This difference of 
$0.5 \mathrm{~dB}$ can be due mainly to the misconnection (i.e weld the connector) of the SMA connector at the different ports.

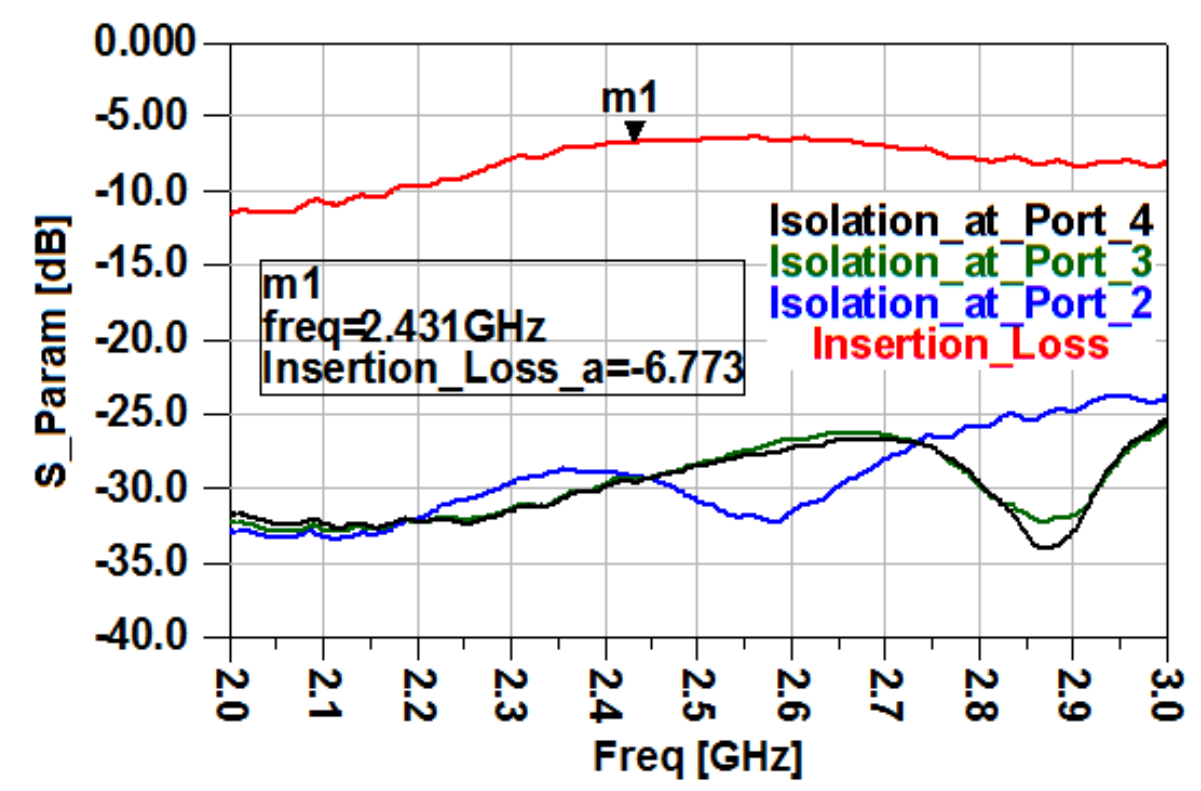

Figure IV.90: Measured S-parameters for case_1 at Pin of O dBm

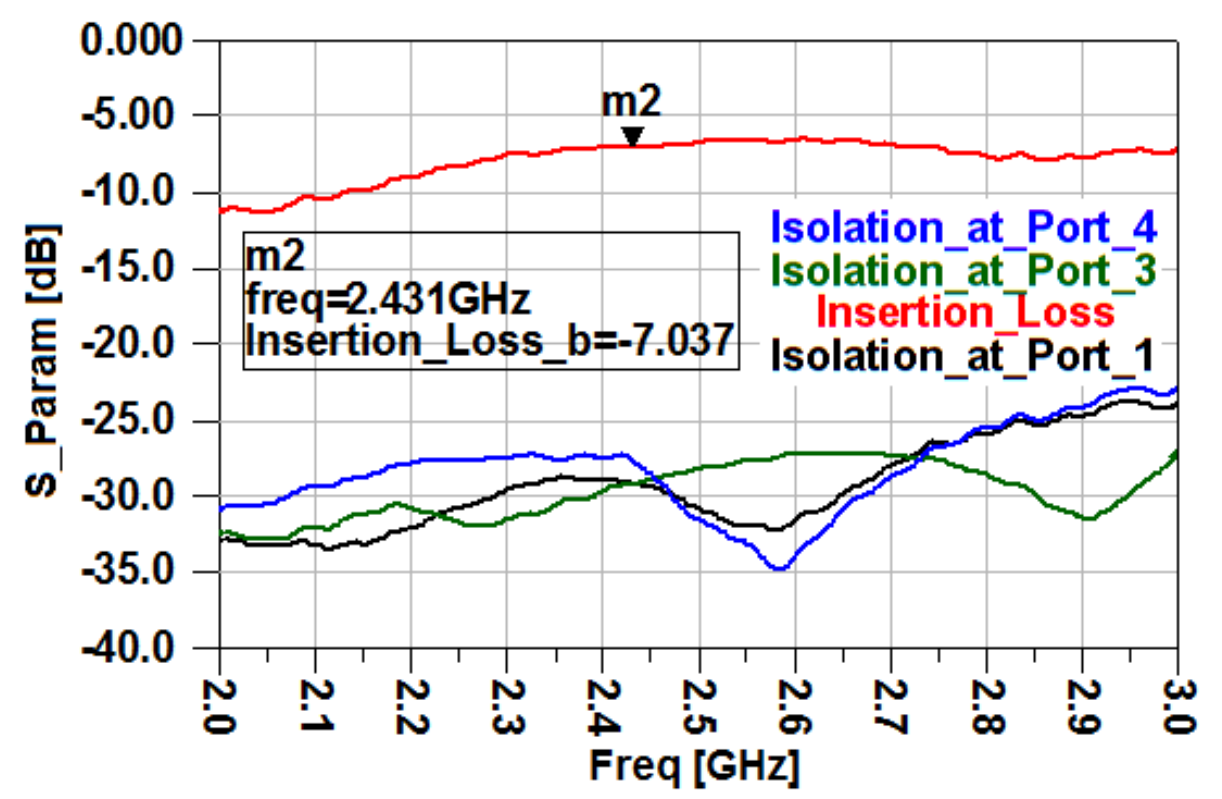

Figure IV.91: Measured S-parameters for case_2 at Pin of $0 \mathrm{dBm}$ 


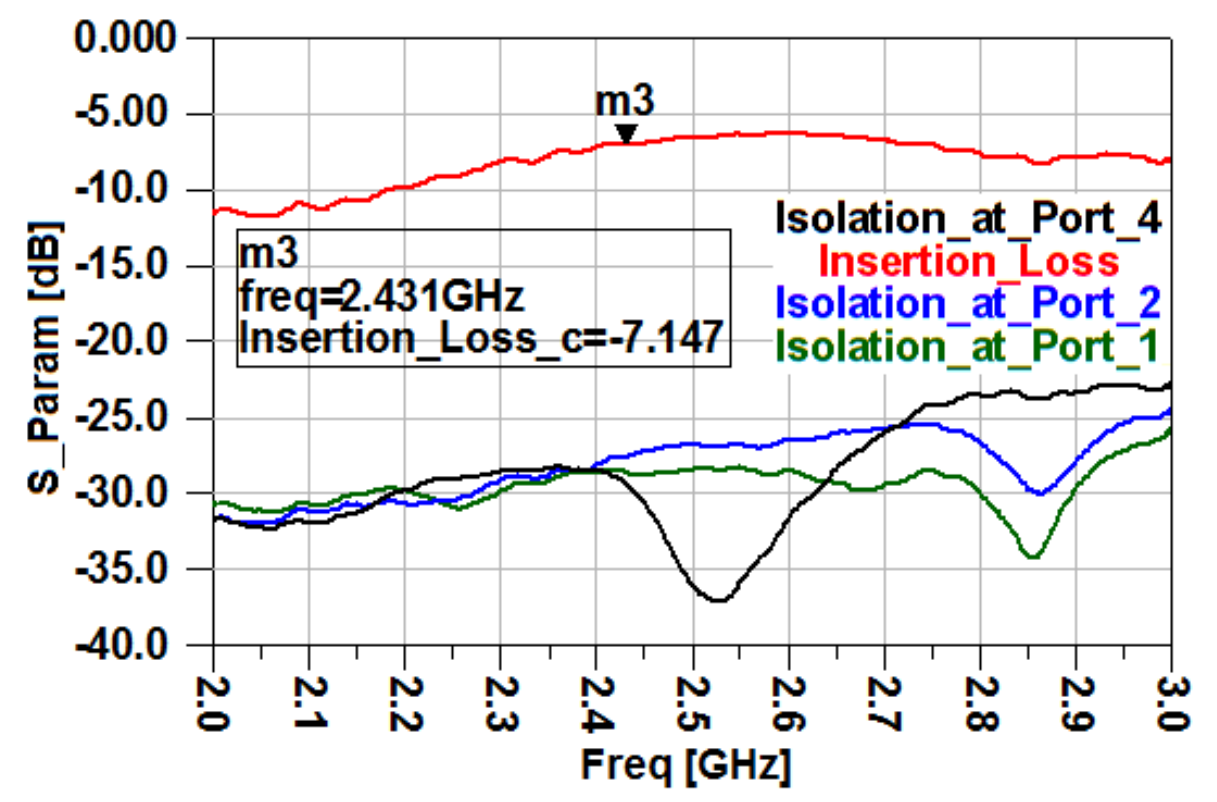

Figure IV.92: Measured S-parameters for case_3 at Pin of O dBm

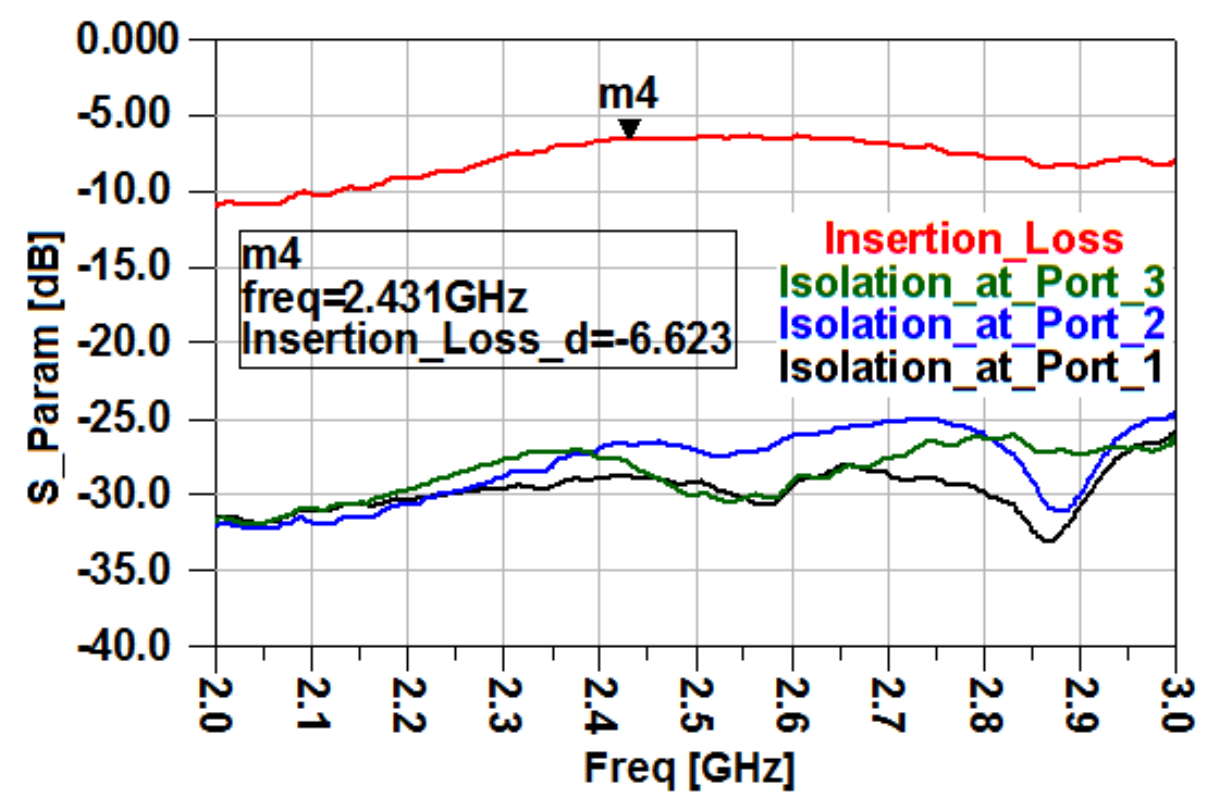

Figure IV.93: Measured S-parameters for case_4 at Pin of O dBm 


\subsubsection{Large Signal Measurements}

The objective here is to demonstrate that GaN-based switch structure can simultaneously achieve reasonably high power handling capability without sacrificing insertion loss and isolation. To satisfy these requirements, a RF power sweep from $0 \mathrm{dBm}$ to $30 \mathrm{dBm}$ at the Port_1 has been done and the insertion losses were measured between Port_1 and Port_2 at these different level of power.

The measured power handling capability of the SPDT switch at $2.43 \mathrm{GHz}$ is shown in Figure IV.94. The insertion loss for ON state was measured with the increasing of input power level at $2.43 \mathrm{GHz}$ for case_1 configuration. As seen the insertion loss remains almost constant up to $16 \mathrm{dBm}$ input power. The measured data shown in Figure IV.94 demonstrates less than $1 \mathrm{~dB}$ of compression for $20 \mathrm{dBm}$ incident $\mathrm{RF}$ power at $2.43 \mathrm{GHz}$. The input power capability of $20 \mathrm{dBm}$ handled by the used GaN transistor can be confirm the potential of using GaN technology for high power regarding the small size of these used devices (as mentioned in Switch modeling section, the devices used are $2 \times 25 \mu \mathrm{m}$ and $2 \times 50 \mu \mathrm{m}$ gate width).

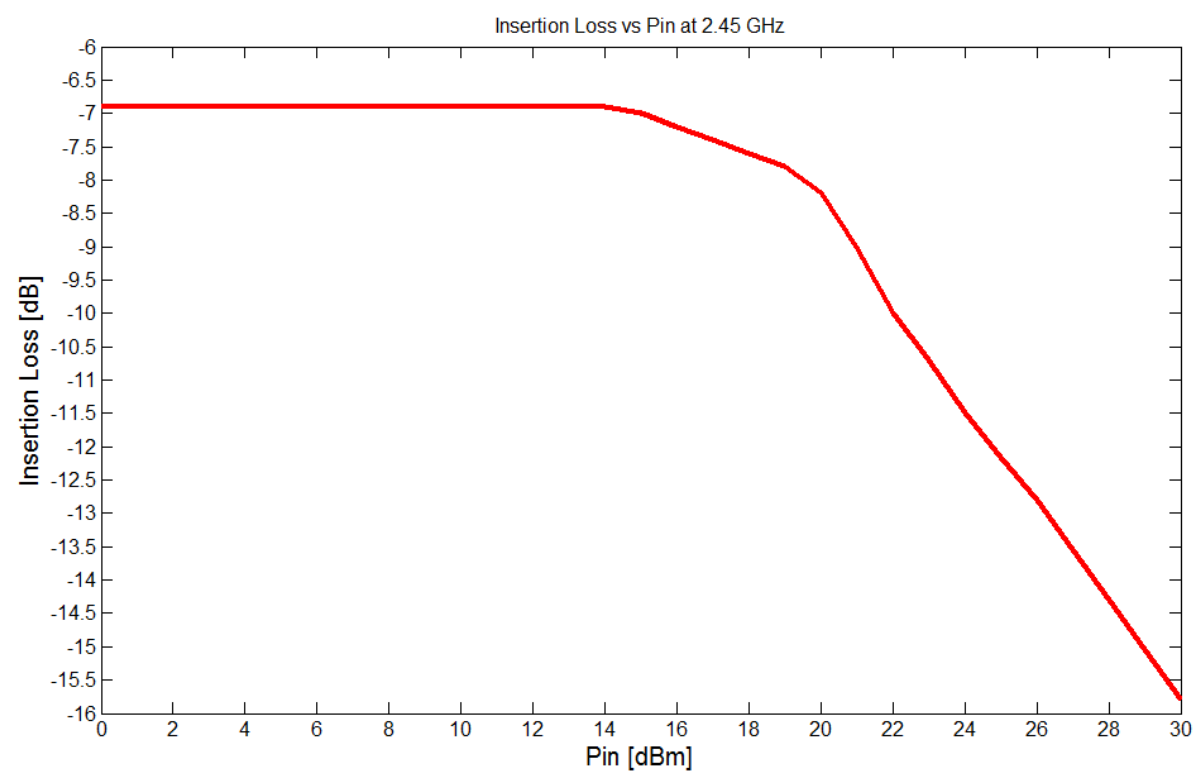

Figure IV.94: Measured insertion loss versus input $R F$ power for case_1 at $2.43 \mathrm{GHz}$ 


\section{Summary}

This preceding chapter deals with implementing reconfigurable designs to validate and verify the use of our developed GaN varactors and switches and demonstrate the feasibility of using GaN technology in tunable RF/microwaves systems. Reconfigurable designs such as phase shifter, $3-\mathrm{dB} 90^{\circ}$ hybrid coupler, oscillator and patch antenna arrays were designed and discussed. The reconfigurablity operation is achieved by using the developed GaN HEMT-based varactor and switch devices as was expected. These GaN circuits were embedded with the listed design by using the wire bonding technique. 


\section{Chapter V}

\section{Conclusion and Future Work}

This thesis is primarily focused on developing models of varactor diodes and switches fabricated using the newest semiconductor technology, gallium nitride (GaN). Varactor and switch devices play a crucial role in design, development and overall performance of wireless communication systems that require reconfigurablity. The thesis can be divided into two major parts: modeling of GaNbased devices such as varactor diodes and switches, and reconfigurable designs where these developed GaN devices are integrated.

The active devices structure studied here are based on GaN HEMT processes offered by National Research Council. Three GaN processes, such as, GaN150v0 with a gate length of $0.15 \mathrm{um}$, GaN500v1 with gate length of $0.5 \mathrm{um}$ and GaN500v2, have been used for manufacturing the varactor diodes and the switches. Based on these three GaN processes, many varactors have been fabricated and characterized. A general small signal and large signal model based on analytic 
equations containing empirical coefficients have been introduced for the first time for the voltage dependency of equivalent capacitance $\left(\mathrm{C}_{\mathrm{Eq}}\right)$ and series resistance $\left(\mathrm{R}_{\mathrm{Eq}}\right)$. For small signal model, all of the developed analytic equations, which describe $R_{\mathrm{Eq}}$ and $\mathrm{C}_{\mathrm{Eq}}$ versus the control $\mathrm{DC}$ voltage $\left(\mathrm{V}_{\mathrm{G}}\right)$, are only bias voltage and device geometry dependent, while for large signal, the influence of $\mathrm{RF}$ power has been taken into account in the proposed analytic expressions describing the behaviour of $\mathrm{R}_{\mathrm{Eq}}$ and $\mathrm{C}_{\mathrm{Eq}}$. These proposed analytical solutions agree remarkably well with the experimentally extracted $\mathrm{C}-\mathrm{V}$ curves for several $\mathrm{RF}$ power and can be used as a general model to represent the nonlinear behavior of GaN based varactor devices. By analyzing the $\mathrm{C}$ $\mathrm{V}$ curve, we conclude that the equivalent series capacitance is dependent on the geometry of fingers. This means that the larger is the device, the higher are the capacitance values. Moreover, it was observed that the extracted capacitance values from varactors with GaN500v2 are at least twice as large as those extracted with the same device size fabricated with GaN500v1 process. The tuning range of the varactor diode is limited, and it was found that over a range of $1 \mathrm{~V}$, the $\mathrm{C}_{\mathrm{Eq}}$ changes rapidly. In addition, an equivalent simple physical circuit model was proposed and simulated, yielding good agreement between the simulated and the measured results. The obtained $\mathrm{Cmax} / \mathrm{Cmin}$ ratios and figures of merit compare favorably with recently reported GaN varactors.

On the other hand, different size single standalone switches and switch structures such as single pole single throw (SPST) and single pole double throw (SPDT) were fabricated using NRC GaN500v2 and were presented. Here only small signal measurement were presented and discussed. It was observed that, with a single transistor in common gate configuration, the isolation is good at low frequencies but quickly drops with increasing frequency. In addition, it was observed that the more the device is large, the lower the insertion loss achieved but the poorer the isolation will be. A trade-off between insertion loss and isolation has been made to choose the single transistors that will be used for designing switch structures such as SPST and SPDT which could improve the performances at high frequencies. 
Comparing to the standalone device, it was revealed that with SPST structure, the isolation is improved while the insertion loss slightly dropped by $-1 \mathrm{~dB}$. With this proposed topology, it is still a challenge to meet good performance at our higher frequency. Another topology based on SPDT was proposed to improve more the performances. As was expected with this topology, some improvement in isolation is achieved compared to SPST, while the insertion loss is increased by almost $-1 \mathrm{~dB}$. For example, at $2.45 \mathrm{GHz}$ (frequency of interest) isolation better than $-25 \mathrm{~dB}$ and an insertion loss of $-3 \mathrm{~dB}$ were achieved. However, the SPDT structure performances degrade with frequency. Moreover, based on these small signal measurements, a model was introduced. In addition, the fabricated SPDT switch structure will be integrated with antenna system to demonstrate the ability of using GaN devices for a beam switching antenna array.

Finally, in this thesis, the use of GaN on highly efficient reconfigurable designs for broadband RF/microwave applications operating below $10 \mathrm{GHz}$ was demonstrated. The varactors and switches used for tunable purpose were firstly fabricated, characterized and then modeled. The proposed models were used to achieve the reconfigurability of $\mathrm{RF} /$ microwave circuits. Tunable phase shifter, reconfigurable $3-\mathrm{dB} 90^{\circ}$ hybrid coupler, tunable frequency oscillator, beam switching antenna array and matching reconfigurable patch antenna were developed along with the development of GaN modeling varactors and switches, and were presented as applications results of this thesis research.

Using GaN150v0 process, two reconfigurable $\mathrm{RF}$ designs have been designed and reported. Firstly, a GaN phase shifter design based on reflective topology has been described, and only simulation results were presented. First, the 3- $\mathrm{dB} 90^{\circ}$ hybrid coupler has been designed and simulated using NRC GaN150 PDK. Then two developed GaN varactors were placed on the two coupler's output ports as loads. Under different bias conditions varying from $-3.5 \mathrm{~V}$ to $-5.5 \mathrm{~V}$, the return loss of the phase shifter is better than $20 \mathrm{~dB}$ across a $1 \mathrm{GHz}$ bandwidth centered at $10 \mathrm{GHz}$, 
while good match is achievable over this range of $9.5-10.5 \mathrm{GHz}$. Worst case of the insertion loss is about $-3 \mathrm{~dB}$ at $10 \mathrm{GHz}$ and occurs at a bias point of $-4.3 \mathrm{~V}$ where the equivalent resistor of the used varactors is significantly high. Therefore, a continuously variable phase-shift range of $80^{\circ}$ is achieved at $10 \mathrm{GHz}$. Secondly, a varactor tunable broadband quadrature hybrid coupler has been investigated and simulated from $\mathrm{X}$-band to $\mathrm{Ku}$-band frequencies applications, and only simulated results were reported. First, a static coupler has been designed to meet the specifications of a typical quadrature hybrid coupler (3-dB insertion loss, good return and isolation loss, and $90^{\circ}$ phase difference between coupled and through ports) at 10 GHz. Then, a tunable broadband coupler has been designed by inserting varactor diodes as shunt capacitance elements in order to control the operating frequency. This design occupies an area of $1.35 \times 0.9 \mathrm{~mm}^{2}$ and good simulation results have been demonstrated. This novel varactor reconfigurable hybrid coupler is proposed to overcome the need of high power tunable $\mathrm{RF} /$ microwave circuits which are required for multi-standard and multiservice applications. By changing the bias voltage of the varactor to control the resonance condition of parallel LC resonator, this proposed hybrid coupler can provide the desired operating frequency from $10 \mathrm{GHz}$ to $16 \mathrm{GHz}$. Over this bandwidth, the overall return loss is better than $-13.5 \mathrm{~dB}$ over a tuning range $1 \mathrm{~V}$ while the isolation is below than $-14 \mathrm{~dB}$. In addition, the simulated magnitude of S21 parameter is close to $-3.5 \mathrm{~dB}$ and is getting poorer as varactor capacitance values decreases. A worst value of $-5 \mathrm{~dB}$ is achieved at the minimum capacitance value. While at port 3, the $\mathrm{S} 31$ parameter behaves as $\mathrm{S} 21$ but in a reverse way. As a result, the amplitude imbalance is varying from $-0.2 \mathrm{~dB}$ at $10 \mathrm{GHz}$ and -2 $\mathrm{dB}$ at $16 \mathrm{GHz}$. A minimum well-balanced quadrature phase of $86^{\circ}$ is obtained between the coupled port 2 and the through port 3 . This phase is increasing and getting closer to $92^{\circ}$ as varactor capacitor values are decreasing.

Using GaN500v2 process, a tunable frequency GaN oscillator was designed by loading symmetrically the feedback loop, and was presented. For tuning purpose, the previous developed GaN varactors were used. The oscillation frequency values 
change significantly and a minimum oscillation frequency of $7.8 \mathrm{GHz}$ was achieved when $\mathrm{C}_{\mathrm{Eq}}$ is at its maximum for $\mathrm{V}_{\mathrm{G}}$ above $-3.5 \mathrm{~V}$, and a maximum oscillation frequency of $8.6 \mathrm{GHz}$ was simulated when $\mathrm{C}_{\mathrm{Eq}}$ is at its minimum for $\mathrm{V}_{\mathrm{G}}$ bellow $-4.5 \mathrm{~V}$. While, when $\mathrm{V}_{\mathrm{G}}$ varies from $-5 \mathrm{~V}$ to $-3.7 \mathrm{~V}$, the oscillation frequency values change significantly and a tuning bandwidth of $0.8 \mathrm{GHz}$ is obtained, corresponding to a tuning range about $10 \%$. The output power is about $20.5 \mathrm{dBm}$ and almost stable over all bias levels with a slight variation over range (2) when $V_{G}$ varies while a good phase noise value of $-112 \mathrm{dBc} / \mathrm{Hz}$ is met at $100 \mathrm{KHz}$. Also three reconfigurable antennas were designed and presented. The first design is a simple element square loop coplanar antenna printed onto glass substrate. The frequency tunability has been achieved by loading the previous developed GaN varactor diode. The antenna was electronically tuned by varying the varactor bias voltage applied at the gate, $\mathrm{V}_{\mathrm{G}}$. As reposted, the minimum and the maximum values of the measured input return loss are, respectively, $-6.9 \mathrm{~dB}$ and $-7.1 \mathrm{~dB}$ over the bandwidth from $7.53 \mathrm{GHz}$ to $7.85 \mathrm{GHz}$ corresponding to a tunability up to $4 \%$ narrow bandwidth inside. This frequency tunability is achieved by using a developed GaN varactor. Another frequency reconfigurability antenna was described and presented. The used structure is based on aperture coupled microstrip patch antenna feed technique. The antenna structure with overall dimensions $\mathrm{L}$ of $50 \mathrm{~mm}$ and $\mathrm{W}$ of $50 \mathrm{~mm}$ consists of two dielectric layers: a slot layer and a simple element patch layer and between them an air-gap layer of $1 \mathrm{~mm}$. As presented, the minimum and the maximum values of the simulated input return loss are, respectively, $-48 \mathrm{~dB}$ and $-13 \mathrm{~dB}$ over a narrow bandwidth from $9.34 \mathrm{GHz}$ to $9.5 \mathrm{GHz}$. This tunability is achieved by integrating a developed varactor with the antenna design. The last reported reconfigurable antenna design is an electronically beam steering antenna using the developed SPDT switch structure is proposed for applications that operate at $2.45 \mathrm{GHz}$ frequency. The idea here is to verify the developed GaN SPDT switch structure fabricated in NRC GaN500v2 process, for this a global system with four patch arrays antenna with beam steering over the $\mathrm{H}$ plane for four $\Phi$ possibilities such as, $0^{\circ}, 90^{\circ}, 180^{\circ}$ and $270^{\circ}$ is designed. 
With the use of this structure, the global array can steer its beam by a single control DC voltage applied at the different single switches constituting the SPDT structure.

Some contributions to the field of microwave integrated and reconfigurable circuit designs have been made from this thesis research. As mentioned in Chapter III, one journal paper [149] has been published and one other has been submitted [151]. A conference paper has also been presented at the European Microwave Conference in Paris in 2015 [150]. Furthermore, a part of the work of the beam steering patch antenna arrays using GaN SPDT switch that was presented in Chapter IV section 4 will be presented at the European Conference on Antennas and Propagation in Paris in 2017

[152] and also based on the antenna work one journal paper is expecting to be submitted for publication.

Despite all the obstacles that we faced during the manufacturing of the GaN design (such as, runs cancelled, chips dicing ...), we succeed to overcome some of them and develop GaN varactor and switch devices which were later integrated with PCB designs to achieve reconfigurability.

There are several areas related to this thesis which are ripe for study. First, if the required equipment became available, large-signal GaN device modeling, particularly for varactor switch devices is an area which should be developed. Improved performance in large signal operation and going higher in frequency of these devices can be expected when improved commercial NRC GaN150 become fully available.

Initially, a NRC GaN150 run was planned and highly miniaturized phase shifter and reconfigurable hybrid coupler designs were planned to be fabricated and tested. Unfortunately these designs have not been manufactured because the run has been cancelled by NRC. So future work could be include both phase shifter and reconfigurable hybrid coupler using the advanced developed version of NRC GaN150 
process. Also it was expected to develop a frequency tunable antenna feeder such as annular slot antenna using varactor diode for reconfigurability. This feeder can be used to tune the frequency of an antenna that is fabricated by using a low cost substrate. 


\section{Bibliography}

[1] D. W. Runton, B. Trabert, J. B. Shealy, and R. Vetury, "History of GaN: HighPower RF Gallium Nitride (GaN) from Infancy to Manufacturable Process and Beyond," IEEE Microwave Magazine, vol. 14, no. 3, pp. 82-93, imsspecialissuemay 2013.

[2] T. Ross, G. Cormier, and K. Hettak, "Gallium nitride hybrid microwave circuits for low-noise applications," in 2010 14th International Symposium on Antenna Technology and Applied Electromagnetics the American Electromagnetics Conference (ANTEM-AMEREM), 2010, pp. 1-4.

[3] U. Johannsen, M. I. Kazim, A. B. Smolders, and M. H. A. J. Herben, "Modular antenna array concept for millimeter-wave beam-steering applications," ProgresS in Electromagnetics Research Symposium Proceedings, Stockholm, Sweden, Aug. 12-15, 2013.

[4] K. Joshin, T. Kikkawa, S. Masuda and K. Watanabe, "Outlook for GaN HEMT technology”, Fujitsu Sci. Tech. J.50, 138-143. 2014.

[5] N. J. Kolias, "Recent advances in Ga N MMIC technology," in 2015 IEEE Custom Integrated Circuits Conference (CICC), 2015, pp. 1-5.

[6] G. Callet, " Caractérisation et Modélisation de Transistors HEMT AlGaN/GaN et InAlN/GaN pour l'Amplification de puissance en Radio-Fréquences," $P h D$ Dissertation. University of Limoges. 2011.

[7] W. Saito, Y. Takada, M. Kuraguchi, K. Tsuda, I. Omura, and T. Ogura, "600V AlGaN/GaN power-HEMT: design, fabrication and demonstration on high voltage DC-DC converter," in Electron Devices Meeting, 2003. IEDM '03 Technical Digest. IEEE International, 2003, p. 23.7.1-23.7.4.

[8] J. Delaine, P. O. Jeannin, D. Frey, and K. Guepratte, "High frequency DC-DC converter using GaN device," in 2012 Twenty-Seventh Annual IEEE Applied Power Electronics Conference and Exposition (APEC), 2012, pp. 1754-1761.

[9] A. Brown, K. Brown, J. Chen, K. C. Hwang, N. Kolias, and R. Scott, "W-band GaN power amplifier MMICs," in Microwave Symposium Digest (MTT), 2011 IEEE MTT-S International, 2011, pp. 1-4.

[10] Y. Nakasha, S. Masuda, K. Makiyama, T. Ohki, M. Kanamura, N. Okamoto, T. Tajima, T. Seino, H. Shigematsu, K. Imanishi, T. Kikkawa, K. Joshin, and N. Hara, "E-Band 85-mW Oscillator and 1.3-W Amplifier ICs Using 0.12 \#181;m GaN HEMTs for Millimeter-Wave Transceivers," in 2010 IEEE Compound Semiconductor Integrated Circuit Symposium (CSICS), 2010, pp. 1-4.

[11] M. Micovic, A. Kurdoghlian, K. Shinohara, I. Milosavljevic, S. D. Burnham, M. Hu, A. L. Corrion, W. S. Wong, A. Schmitz, P. B. Hashimoto, P. J. Willadsen, D. H. Chow, A. Fung, R. H. Lin, L. Samoska, P. P. Kangaslahti, B. H. Lambrigtsen, and P. F. Goldsmith, "W-band GaN MMIC with $842 \mathrm{~mW}$ output power at $88 \mathrm{GHz}$," in Microwave Symposium Digest (MTT), 2010 IEEE MTT-S International, 2010, pp. $1-1$. 
[12] J. W. Milligan, S. Sheppard, W. Pribble, Y. F. Wu, G. Muller, and J. W. Palmour, "SiC and GaN Wide Bandgap Device Technology Overview," in 2007 IEEE Radar Conference, 2007, pp. 960-964.

[13] W. Saito, Y. Takada, M. Kuraguchi, K. Tsuda, I. Omura, T. Ogura, and H. Ohashi, "High breakdown voltage AlGaN-GaN power-HEMT design and high current density switching behavior," IEEE Transactions on Electron Devices, vol. 50, no. 12, pp. 2528-2531, Dec. 2003.

[14] T. Zimmermann, D. Deen, Y. Cao, J. Simon, P. Fay, D. Jena, and H. G. Xing, "AlN/GaN Insulated-Gate HEMTs With $2.3 \mathrm{~A} / \mathrm{mm}$ Output Current and $480 \mathrm{mS} / \mathrm{mm}$ Transconductance," IEEE Electron Device Letters, vol. 29, no. 7, pp. 661-664, Jul. 2008.

[15] Y. f Wu, M. Moore, A. Saxler, T. Wisleder, and P. Parikh, "40-W/mm Double Field-plated GaN HEMTs," in 2006 64th Device Research Conference, 2006, pp. $151-152$.

[16] Discrete GaAs pHEMT, TGF2018, Rev. D, TriQuint Semiconductor, Nov. 2013.

[17] Discrete power GaN on SiC HEMT, TGF2023-2-01, Rev. C, TriQuint Semiconductor, Sep. 2013.

[18] Q. Rüdiger, "Gallium Nitride Electronics," vol. 96. Berlin, Heidelberg: Springer Berlin Heidelberg, 2008.

[19] Md. Tanvir Hasan "Mechanism and Suppression of Current Collapse in AlGaN/GaN High Electron Mobility Transistors". PhD Dissertation. University of Fukui, Fukui, Japan, 2013.

[20] R. Vetury, N. Q. Zhang, S. Keller, and U. K. Mishra, "The impact of surface states on the DC and RF characteristics of AlGaN/GaN HFETs," IEEE Transactions on Electron Devices, vol. 48, no. 3, pp. 560-566, Mar. 2001.

[21] O. Mitrofanov and M. Manfra, "Mechanisms of gate lag in GaN/AlGaN/GaN high electron mobility transistors," Superlattices and Microstructures, vol. 34, no. 1-2, pp. 33-53, Jul. 2003.

[22] P. B. Klein and S. C. Binari, "Photoionization spectroscopy of deep defects responsible for current collapse in nitride-based field effect transistors," Journal of Physics: Condensed Matter, 15 (2003) R1641-R1667. PII: S0953-8984(03)58996-2

[23] M. Faqir, G. Verzellesi, A. Chini, F. Fantini, F. Danesin, G. Meneghesso, E. Zanoni, and C. Dua, "Mechanisms of RF Current Collapse in AlGaN \#x2013;GaN High Electron Mobility Transistors," IEEE Transactions on Device and Materials Reliability, vol. 8, no. 2, pp. 240-247, Jun. 2008.

[24] S. Nakamura, M. Senoh, S. Nagahama, N. Iwasa, T. Yamada, T. Matsushita, H. Kiyoku, Y. Sugimoto, T. Kozaki, H. Umemoto, M. Sano, and K. Chocho, "Continuous-wave operation of InGaN/GaN/AlGaN based laser diodes grown on GaN substrates", Applied Physics. Letters, vol. 72, no. 16, Apr. 1998, pp. 2014-2016.

[25] T. Paskova, D. A. Hanser, and K. R. Evans, "GaN Substrates for III-Nitride Devices," Proceedings of the IEEE, vol. 98, no. 7, pp. 1324-1338, Jul. 2010. 
[26] Hageman, P.R.; Kirilyuk, V.; Corbeek, W.H.M.; Weyher, J.L.; Lucznik, B.; Bockowski, M.; Porowski, S.; Müller, S. "Thick GaN layers grown by hydride vaporphase epitaxy: hetero- versus homo-epitaxy". Journal of Crystal Growth 255 (2003), No.3-4, pp.241-249.

[27] R. Dwilinski, R. Doradzinski, J. Garczynski, L. P. Sierzputowski, A. Puchalski, Y. Kanbara, K. Yagi, H. Minakuchi, and H. Hayashi, "Bulk ammonothermal GaN", Journal of Crystal Growth, vol. 311, no. 10, May 2009, pp. 3015-3018.

[28] T. Hashimoto, E. Letts, M. Ikari, and Y. Nojima, "Improvement of crystal quality in ammonothermal growth of bulk GaN", Journal of Crystal Growth, vol. 312 , no. 18, Sep. 2010, pp. 2503-2506.

[29] B. Łucznik, B. Pastuszka, I. Grzegory, M. Bockowski, G. Kamler, E. LitwinStaszewska, and S. Porowski, "Deposition of thick GaN layers by HVPE on the pressure grown GaN substrates", Journal of Crystal Growth, vol. 281, no. 1, Jul. 2005, pp. 38-46.

[30] T. N. Ross. "Gallium Nitride Phase Shifters". PhD Dissertation. March, 2015. Carleton University, Ottawa, Canada.

[31] "Gallium Nitride MMIC Foundry Design Manual GaN500v2," Canadian Photonics Fabrication Center. National Research Council Canada, 2013.

[32] H. Tang, J. B. Webb, and J. A. Bardwell. "The ammonia- MBE approach for high electron mobility in GaN epilayers and AlGaN/GaN HFET structures".

[33] J. Bardwell, T. Lester, H. Tang, B. Surridge, A. Harrison, and A. Lau, "AlGaN/GaN Transistors and Circuits," ECS Trans., vol. 16, no. 7, pp. 193-198, Oct. 2008.

[34] T. Edwards, "Semiconductor Technology Trends for Phased Array Antenna Power Amplifiers," in 2006 European Radar Conference, 2006, pp. 269-272.

[35] T. Ohira, Y. Suzuki, H. Ogawa, and K. Kamitsuna, "Megalithic microwave signal processing for phased-array beamforming and steering," IEEE Transactions on Microwave Theory and Techniques, vol. 45, no. 12, pp. 2324-2332, Dec. 1997.

[36] L. F. Eastman and U. K. Mishra, "The toughest transistor yet [GaN transistors],” IEEE Spectrum, vol. 39, no. 5, pp. 28-33, May 2002.

[37] B. Heying, W. Luo, I. Smorchkova, S. Din, and M. Wojtowicz, "Reliable GaN HEMTS for high frequency applications," in Microwave Symposium Digest (MTT), 2010 IEEE MTT-S International, 2010, pp. 1-1.

[38] M. Oppermann, F. Thurow, and B. Bunz, "GaN/SiC MMICs and packaging for use in future transmit / receive modules," in Electronics System-Integration Technology Conference (ESTC), 2014, 2014, pp. 1-4.

[39] C. Lin, H. Liu, and Y. Zheng, "Application of GaN high power chips in T/R modules," in 2013 Proceedings of the International Symposium on Antennas Propagation (ISAP), 2013, vol. 1, pp. 515-518. 
[40] N. J. Kolias and M. T. Borkowski, "The development of T/R modules for radar applications," in Microwave Symposium Digest (MTT), 2012 IEEE MTT-S International, 2012, pp. 1-3.

[41] H. Yukawa, M. Hangai, H. Mizutani, K. Yamanaka, M. Abe, A. Inoue, and M. Miyazaki, "Q-band gaas MMIC modules for active phased array antenna systems," in Microwave Conference, 2009. EuMC 2009. European, 2009, pp. 464-467.

[42] D. Baccello, M. D'Antoni, B. Orobello, and E. Sperduti, "Miniaturised low cost solid state 4W TXRX common leg for 6 \#x00F7;18 GHz phased array," in Microwave Symposium Digest (MTT), 2010 IEEE MTT-S International, 2010, pp. 401-403.

[43] P. Schuh, H. Sledzik, R. Reber, A. Fleckenstein, R. Leberer, M. Oppermann, R. Quay, F. van Raay, M. Seelmann-Eggebert, R. Kiefer, and M. Mikulla, "GaN MMIC based T/R-Module Front-End for X-Band Applications," in Microwave Integrated Circuit Conference, 2008. EuMIC 2008. European, 2008, pp. 274-277.

[44] S. Masuda, M. Yamada, T. Ohki, K. Makiyama, N. Okamoto, Y. Nakasha, K. Imanishi, T. Kikkawa, and H. Shigematsu, "C-Ku band GaN MMIC T/R frontend module using multilayer ceramics technology," in Microwave Symposium Digest (MTT), 2011 IEEE MTT-S International, 2011, pp. 1-4.

[45] D. Gustafsson, J. C. Cahuana, D. Kuylenstierna, I. Angelov, and C. Fager, "A GaN MMIC Modified Doherty PA With Large Bandwidth and Reconfigurable Efficiency," IEEE Transactions on Microwave Theory and Techniques, vol. 62, no. 12, pp. 3006-3016, Dec. 2014.

[46] V. Jantarachote, N. Sirivech, and S. Chalermwisutkul, "Frequency band and operating class reconfigurable GaN HEMT power amplifier for sustainable wireless communications and energy applications," in The 20th Asia-Pacific Conference on Communication (APCC2014), 2014, pp. 156-160.

[47] O. L. Chlieh, W. T. Khan, and J. Papapolymerou, "Microfluidically reconfigurable GaN power amplifier on multilayer organic substrate for s-band and c-band applications," in 2015 IEEE MTT-S International Microwave Symposium, 2015, pp. 1-4.

[48] R. Pengelly, S. Sheppard, T. Smith, B. Pribble, S. Wood and C. Platis, "Commercial GaN Devices for Switching and Low Noise Applications", $C S$ MANTECH Conference, Palm Springs, CA, USA, May 2011.

[49] X. Lan, M. Wojtowicz, M. Truong, F. Fong, M. Kintis, B. Heying, I. Smorchkova, and Y. C. Chen, "A V-Band Monolithic AlGaN/GaN VCO," IEEE Microwave and Wireless Components Letters, vol. 18, no. 6, pp. 407-409, Jun. 2008.

[50] T. N. Ross, G. Cormier, K. Hettak, and J. S. Wight, "High-power X-band GaN switched-filter phase shifter," in 2014 IEEE MTT-S International Microwave Symposium (IMS2014), 2014, pp. 1-4.

[51] K. Andersson, V. Desmaris, J. Eriksson, N. Rorsman, and H. Zirath, "C-band linear resistive wide bandgap FET mixers," in Microwave Symposium Digest, 2003 IEEE MTT-S International, 2003, vol. 2, pp. 1303-1306 vol.2. 
[52] C. Jin, E. Okada, M. Faucher, D. Ducatteau, M. Zaknoune, and D. Pavlidis, "A GaN Schottky Diode-based analog phase shifter MMIC," in European Microwave Integrated Circuit Conference (EuMIC), 2014 9th, 2014, pp. 96-99.

[53] M. Marso, J. Bernat, A. Wolter, P. Javorka, A. Fox, and P. Kordos, "MSM diodes based on an AlGaN/GaN HEMT layer structure for varactor and photodiode application," in The Fourth International Conference on Advanced Semiconductor Devices and Microsystems, 2002, 2002, pp. 295-298.

[54] C. S. Chu, Y. Zhou, K. J. Chen, and K. M. Lau, "Q-factor characterization of RF GaN-based metal-semiconductor-metal planar interdigitated varactor," IEEE Electron Device Letters, vol. 26, no. 7, pp. 432-434, Jul. 2005.

[55] L. B. Chang, S. C. Wang, S. L. Lin, M. Das, Y. C. Ferng, M. J. Cheng, S. T. Chou, and L. Chow, "Fast surge suppression by inserting an AlGaN/GaN-based varactor," in 2012 2nd International Conference on Consumer Electronics, Communications and Networks (CECNet), 2012, pp. 3251-3253.

[56] C.-Y. Tien; P.-Y. Kuei ; Liann-Be Chang; C.-P. Hsu. "Capacitance Swing and Capacitance Ratio of GaN-Based Metal-Semiconductor-Metal Two-Dimensional Electron Gas Varactor with Different Dielectric Films", Journal of Electrical Engineering and Technology 10(4):1721-1726. July 2015.

[57] C. Jin, D. Pavlidis, and L. Considine, "A novel GaN-based high frequency varactor diode," in Microwave Integrated Circuits Conference (EuMIC), 2010 European, 2010, pp. 118-121.

[58] W. Lu, L. Wang, S. Gu, D. P. R. Aplin, D. M. Estrada, P. K. L. Yu, and P. M. Asbeck, "InGaN/GaN Schottky Diodes With Enhanced Voltage Handling Capability for Varactor Applications," IEEE Electron Device Letters, vol. 31, no. 10, pp. 11191121, Oct. 2010.

[59] W. Lu, L. Wang, S. Gu, P. M. Asbeck, and P. K. L. Yu, "Critical design considerations for GaN-based microwave power varactors," in 2012 IEEE International Conference on Electron Devices and Solid State Circuit (EDSSC), 2012, pp. 1-4.

[60] S.Arulkumaran, T. Egawa, H. Ishikawa, T. Jimbo, and Y. Sano, "Surface passivation effects on AlGaN/GaN high-electron-mobility transistors with $\mathrm{SiO} 2$, Si3N4, and silicon oxynitride," Appl. Phys. Lett., vol. 84, pp. 613-615, 2004.

[61] J. S. Lee, J. W. Kim, J. H. Lee, C. S. Kim, J. E. Oh, M. W. Shin, and J. H. Lee, "Reduction of current collapse in AlGaN/GaN HFETs using AlN interfacial layer," Electronics Letters, vol. 39, no. 9, pp. 750-752, May 2003.

[62] Y. F. Wu, A. Saxler, M. Moore, R. P. Smith, S. Sheppard, P. M. Chavarkar, T. Wisleder, U. K. Mishra, and P. Parikh, "30-W/mm GaN HEMTs by field plate optimization," IEEE Electron Device Letters, vol. 25, no. 3, pp. 117-119, Mar. 2004.

[63] A. P. Edwards, J. A. Mittereder, S. C. Binari, D. S. Katzer, D. F. Storm, and J. A. Roussos, "Improved reliability of AlGaN-GaN HEMTs using an NH3 plasma treatment prior to SiN passivation," IEEE Electron Device Letters, vol. 26, no. 4, pp. 225-227, Apr. 2005. 
[64] N. Miura, T. Nanjo, M. Suita, T. Oishi, Y. Abe, T. Ozeki, H. Ishikawa, T. Egawa, and T. Jimbo, "Thermal annealing effects on Ni/Au based Schottky contacts on nGaN and AlGaN/GaN with insertion of high work function metal," Solid-State Electronics, vol. 48, no. 5, pp. 689-695, May 2004.

[65] Y. Takei, M. Kamiya, K. Tsutsui, W. Saito, K. Kakushima, H. Wakabayashi, Y. Kataoka, and H. Iwai, "Reduction of contact resistance on AlGaN/GaN HEMT structures introducing uneven AlGaN layers," Phys. Status Solidi A, vol. 212, no. 5, pp. 1104-1109, May 2015.

[66] M. Okamoto, K. Kakushima, Y. Kataoka, A. Nishiyama, N. Sugii, H. Wakabayashi, K. Tsutsui, H. Iwai, and W. Saito, "An ohmic contact process for AlGaN/GaN structures using TiSi2 electrodes," in 2013 IEEE Workshop on Wide Bandgap Power Devices and Applications (WiPDA), 2013, pp. 159-161.

[67] W. Saito, I. Omura, T. Ogura, and H. Ohashi, "Theoretical limit estimation of lateral wide band-gap semiconductor power-switching device," Solid-State Electronics, vol. 48, no. 9, pp. 1555-1562, Sep. 2004.

[68] S. Lucyszyn, G. Green, and I. D. Robertson, "Accurate millimeter-wave large signal modeling of planar Schottky varactor diodes," in Microwave Symposium Digest, 1992., IEEE MTT-S International, 1992, pp. 259-262 vol.1.

[69] W. Ciccognani, M. Ferrari, and E. Limiti, "High isolation microstrip GaNHEMT Single-FET Switch," Int J RF and Microwave Comp Aid Eng, vol. 20, no. 4, pp. 391-398, Jul. 2010.

[70] A. Bettidi, A. Cetronio, W. Ciccognani, M. De Dominicis, C. Lanzieri, E. Limiti, A. Manna, M. Peroni, C. Proietti, and P. Romanini, "High power GaN-HEMT SPDT switches for microwave applications," Int J RF and Microwave Comp Aid Eng, vol. 19, no. 5, pp. 598-606, Sep. 2009.

[71] L. Devlin, "The design of integrated switches and phase shifters," in Design of RFICS and MMICS (Ref. No. 1999/158), IEE Tutorial Colloquium, 1999, p. 2/1-2/14.

[72] N. Q. Zhang, B. Moran, S. P. DenBaars, U. K. Mishra, X. W. Wang, and T. P. $\mathrm{Ma}$, "Effects of surface traps on breakdown voltage and switching speed of GaN power switching HEMTs," in Electron Devices Meeting, 2001. IEDM '01. Technical Digest. International, 2001, p. 25.5.1-25.5.4.

[73] H. Ishida, Y. Hirose, T. Murata, A. Kanda, Y. Ikeda, T. Matsuno, K. Inoue, Y. Uemoto, T. Tanaka, T. Egawa, and D. Ueda, "A high power Tx/Rx switch IC using AlGaN/GaN HFETs," in Electron Devices Meeting, 2003. IEDM '03 Technical Digest. IEEE International, 2003, p. 23.6.1-23.6.4.

[74] H. Ishida, Y. Hirose, T. Murata, Y. Ikeda, T. Matsuno, K. Inoue, Y. Uemoto, T. Tanaka, T. Egawa, and D. Ueda, "A high-power RF switch IC using AlGaN/GaN HFETs with single-stage configuration," IEEE Transactions on Electron Devices, vol. 52, no. 8, pp. 1893-1899, Aug. 2005.

[75] V. Kaper, R. Thompson, T. Prunty and J. R. Shealy, "Monolithic AlGaN/GaN HEMT SPDT switch," 12th GAAS Symposium. Amsterdam, 2004. 
[76] U. K. Mishra, P. Parikh, and Y.-F. Wu, "AlGaN/GaN HEMTs-an overview of device operation and applications," Proceedings of the IEEE, vol. 90, no. 6, pp. 10221031, Jun. 2002.

[77] B. Y. Ma, K. S. Boutros, J. B. Hacker, and G. Nagy, "High power AlGaN/GaN Ku-band MMIC SPDT switch and design consideration," in Microwave Symposium Digest, 2008 IEEE MTT-S International, 2008, pp. 1473-1476.

[78] J. Janssen, K. P. Hilton, J. O. Maclean, D. J. Wallis, J. Powell, M. Uren, T. Martin, M. van Heijningen, and F. van Vliet, "X-Band GaN SPDT MMIC with over 25 Watt Linear Power Handling," in Microwave Integrated Circuit Conference, 2008. EuMIC 2008. European, 2008, pp. 190-193.

[79] K. Hettak, T. Ross, N. Irfan, D. Gratton, M. C. E. Yagoub, and J. Wight, "Highpower broadband GaN HEMT SPST/SPDT switches based on resonance inductors and shunt-stacked transistors," in Microwave Integrated Circuits Conference (EuMIC), 2012 7th European, 2012, pp. 215-218.

[80] X. Zheng, J. C. Tremblay, S. E. Huettner, K. P. Ip, T. Papale, and K. L. Lange, "Ka-Band High Power GaN SPDT Switch MMIC," in 2013 IEEE Compound Semiconductor Integrated Circuit Symposium (CSICS), 2013, pp. 1-5.

[81] I. Angelov, H. Zirath, and N. Rosman, "A new empirical nonlinear model for HEMT and MESFET devices," IEEE Transactions on Microwave Theory and Techniques, vol. 40, no. 12, pp. 2258-2266, Dec. 1992.

[82] I. Angelov, N. Rorsman, J. Stenarson, M. Garcia, and H. Zirath, "An empirical table-based FET model," IEEE Transactions on Microwave Theory and Techniques, vol. 47, no. 12, pp. 2350-2357, Dec. 1999.

[83] W. R. Curtice, "Nonlinear modeling of compound semiconductor HEMTs, state of the art," in Microwave Symposium Digest (MTT), 2010 IEEE MTT-S International, 2010, pp. 1-1.

[84] S. C. Binari, K. Ikossi, J. A. Roussos, W. Kruppa, D. Park, H. B. Dietrich, D. D. Koleske, A. E. Wickenden, and R. L. Henry, "Trapping effects and microwave power performance in AlGaN/GaN HEMTs," IEEE Transactions on Electron Devices, vol. 48, no. 3, pp. 465-471, Mar. 2001.

[85] O. Jardel, F. D. Groote, T. Reveyrand, J. C. Jacquet, C. Charbonniaud, J. P. Teyssier, D. Floriot, and R. Quere, "An Electrothermal Model for AlGaN/GaN Power HEMTs Including Trapping Effects to Improve Large-Signal Simulation Results on High VSWR," IEEE Transactions on Microwave Theory and Techniques, vol. 55, no. 12, pp. 2660-2669, Dec. 2007.

[86] F. Lin and G. Kompa, "FET model parameter extraction based on optimization with multiplane data-fitting and bidirectional search-a new concept," IEEE Transactions on Microwave Theory and Techniques, vol. 42, no. 7, pp. 1114-1121, Jul. 1994.

[87] C. van Niekerk and P. Meyer, "Performance and limitations of decompositionbased parameter extraction procedures for FET small-signal models," IEEE Transactions on Microwave Theory and Techniques, vol. 46, no. 11, pp. 1620-1627, Nov. 1998. 
[88] I. Angelov, L. Bengtsson, and M. Garcia, "Extensions of the Chalmers nonlinear HEMT and MESFET model," IEEE Transactions on Microwave Theory and Techniques, vol. 44, no. 10, pp. 1664-1674, Oct. 1996.

[89] L. Dunleavy, C. Baylis, W. Curtice, and R. Connick, "Modeling GaN: Powerful but Challenging," IEEE Microwave Magazine, vol. 11, no. 6, pp. 82-96, Oct. 2010.

[90] T. N. Ross, K. Hettak, G. Cormier, and J. S. Wight, "Design of X-Band GaN Phase Shifters," IEEE Transactions on Microwave Theory and Techniques, vol. 63, no. 1, pp. 244-255, Jan. 2015.

[91] J. A. Pla and W. Struble, "Nonlinear model for predicting intermodulation distortion in GaAs FET RF switch devices," in Microwave Symposium Digest, 1993., IEEE MTT-S International, 1993, pp. 641-644 vol.2.

[92] G. Boeck, D. Plenkowski, R. Circa, M. Otte, B. Heyne, P. Rykaczewski, R. Wittmann, and R. Kakerow, "RF front-end technology for reconfigurable mobile systems," in Microwave and Optoelectronics Conference, 2003. IMOC 2003. Proceedings of the 2003 SBMO/IEEE MTT-S International, 2003, vol. 2, pp. 863868 vol.2.

[93] V. Vidojkovic, J. van der Tang, A. Leeuwenburgh, A. van Roermund, "Adaptive Multi-Standard RF Front-Ends," Springer Netherlands, 2008, pp. 1-16.

[94] F. Eshghabadi, F. Banitorfia, N. M. Noh, A. A. Manaf, M. T. Mustaffa, and O. Sidek, "MEMS-based Reconfigurable CMOS LNAs for Wireless Applications," Trends in Applied Sciences Research, vol. 7, no. 4, pp. 273-284, Apr. 2012.

[95] E. Lourandakis, R. Weigel, H. Mextorf, and R. Knoechel, "Circuit Agility," IEEE Microwave Magazine, vol. 13, no. 1, pp. 111-121, Jan. 2012.

[96] J. MANUEL, "Composants innovants reconfigurables par voie optique pour des applications microondes," PhD Dissertation. University of Limoges. 2011.

[97] G. B. Norris, D. C. Boire, G. S. Onge, C. Wutke, C. Barratt, W. Coughlin, and J. Chickanosky, "A fully monolithic 4-18 GHz digital vector modulator," in Microwave Symposium Digest, 1990., IEEE MTT-S International, 1990, pp. 789792 vol.2.

[98] R. V. Garver, "Broad-Band Diode Phase Shifters," IEEE Transactions on Microwave Theory and Techniques, vol. 20, no. 5, pp. 314-323, May 1972.

[99] K. Hettak, T. Ross, D. Gratton, and J. Wight, "A new type of GaN HEMT based high power high-pass/low-pass phase shifter at X band," in Microwave Symposium Digest (MTT), 2012 IEEE MTT-S International, 2012, pp. 1-3.

[100] T. N. Ross, G. Cormier, K. Hettak, and J. S. Wight, "High-power X-band GaN switched-filter phase shifter," in 2014 IEEE MTT-S International Microwave Symposium (IMS2014), 2014, pp. 1-4.

[101] C. Jin, E. Okada, M. Faucher, D. Ducatteau, M. Zaknoune, and D. Pavlidis, "A GaN Schottky Diode-based analog phase shifter MMIC," in European Microwave Integrated Circuit Conference (EuMIC), 2014 9th, 2014, pp. 96-99. 
[102] R. V. Garver, “360 Varactor Linear Phase Modulator,” IEEE Transactions on Microwave Theory and Techniques, vol. 17, no. 3, pp. 137-147, Mar. 1969.

[103] H. A. Atwater, "Reflection Coefficient Transformations for Phase-Shift Circuits," IEEE Transactions on Microwave Theory and Techniques, vol. 28, no. 6, pp. 563-568, Jun. 1980.

[104] R. C. Frye, S. Kapur, and R. C. Melville, "A 2-GHz quadrature hybrid implemented in CMOS technology," IEEE Journal of Solid-State Circuits, vol. 38, no. 3, pp. 550-555, Mar. 2003.

[105] "Gallium Nitride MMIC Foundry Design Manual GaN150," Canadian Photonics Fabrication Center. National Research Council Canada, 2015

[106] R. C. Waterman, W. Fabian, R. A. Pucel, Y. Tajima, and J. L. Vorhaus, "GaAs monolithic Lange and Wilkinson couplers," IEEE Transactions on Electron Devices, vol. 28, no. 2, pp. 212-216, Feb. 1981.

[107] S. Lucyszyn and I. D. Robertson, "Analog reflection topology building blocks for adaptive microwave signal processing applications," IEEE Transactions on Microwave Theory and Techniques, vol. 43, no. 3, pp. 601-611, Mar. 1995.

[108] C.-L. Chen, W. E. Courtney, L. J. Mahoney, M. J. Manfra, A. Chu, and H. A. Atwater, "A Low-Loss Ku-Band Monolithic Analog Phase Shifter," IEEE Transactions on Microwave Theory and Techniques, vol. 35, no. 3, pp. 315-320, Mar. 1987.

[109] F. Ellinger, R. Vogt, and W. Bachtold, "Compact reflective-type phase-shifter MMIC for C-band using a lumped-element coupler," IEEE Transactions on Microwave Theory and Techniques, vol. 49, no. 5, pp. 913-917, May 2001.

[110] H. Hayashi, T. Nakagawa, and K. Araki, "A miniaturized MMIC analog phase shifter using two quarter-wave-length transmission lines," IEEE Transactions on Microwave Theory and Techniques, vol. 50, no. 1, pp. 150-154, Jan. 2002.

[111] R. Scheeler and Z. Popović, "GaAs MMIC tunable directional coupler," in Microwave Symposium Digest (MTT), 2012 IEEE MTT-S International, 2012, pp. $1-3$.

[112] B. Hur and W. R. Eisenstadt, "Tunable Broadband MMIC Active Directional Coupler," IEEE Transactions on Microwave Theory and Techniques, vol. 61, no. 1, pp. 168-176, Jan. 2013.

[113] R. V. Gatti, A. Ocera, S. Bastioli, L. Marcaccioli, and R. Sorrentino, "A Novel Compact Dual Band Reconfigurable Power Divider for Smart Antenna Systems," in 2007 IEEE/MTT-S International Microwave Symposium, 2007, pp. 423-426.

[114] S. Voinigescu, "High-Frequency Integrated Circuits", Cambridge University Press, 2013.

[115] A. Balandin, S. V. Morozov, S. Cai, R. Li, K. L. Wang, G. Wijeratne, and C. R. Viswanathan, "Low flicker-noise GaN/AlGaN heterostructure field-effect transistors for microwave communications," IEEE Transactions on Microwave Theory and Techniques, vol. 47, no. 8, pp. 1413-1417, Aug. 1999. 
[116] S. Shin, G. Choi, H. Kim, S. Lee, S. Kim, and J. Choi, "Frequency-tunable high-efficiency power oscillator using GaN HEMT," in Microwave Symposium Digest (MTT), 2010 IEEE MTT-S International, 2010, pp. 1-1.

[117] M. Hörberg, L. Szhau, T. N. T. Do, and D. Kuylenstierna, "Phase noise analysis of a tuned-input/tuned-output oscillator based on a GaN HEMT device," in Microwave Conference (EuMC), 2014 44th European, 2014, pp. 1118-1121.

[118] H. Mostardinha, P. M. Cabral, N. B. Carvalho, P. Kurpas, M. Rudolph, J. Würfl, J. C. Pinto, A. Barnes, and F. Garat, "GaN RF oscillator used in space applications," in 2010 Workshop on Integrated Nonlinear Microwave and Millimeter-Wave Circuits (INMMIC), 2010, pp. 50-53.

[119] S. Kim, H. Kim, S. Shin, J. Kim, B. Kim, and J. Choi, "Combined power oscillator using GaN HEMT," in Microwave Symposium Digest (MTT), 2011 IEEE MTT-S International, 2011, pp. 1-1.

[120] C. Bansleben and W. Heinrich, "Compact high-power GaN oscillator with 2.45 GHz differential output," in Microwave Conference (EuMC), 2013 European, 2013, pp. 818-821.

[121] J. B. Shealy, J. A. Smart, and J. R. Shealy, "Low-phase noise AlGaN/GaN FET-based voltage controlled oscillators (VCOs)," IEEE Microwave and Wireless Components Letters, vol. 11, no. 6, pp. 244-245, Jun. 2001.

[122] H. Xu, C. Sanabria, N. K. Pervez, S. Keller, U. K. Mishra, and R. A. York, "Low phase-noise $5 \mathrm{GHz}$ AlGaN/GaN HEMT oscillator integrated with BaxSr1xTiO3 thin films," in Microwave Symposium Digest, 2004 IEEE MTT-S International, 2004, vol. 3, p. 1509-1512 Vol.3.

[123] C. Sanabria, H. Xu, S. Heikman, U. K. Mishra, and R. A. York, "A GaN differential oscillator with improved harmonic performance," IEEE Microwave and Wireless Components Letters, vol. 15, no. 7, pp. 463-465, Jul. 2005.

[124] C. Kong, H. Li, X. Chen, S. Jiang, J. Zhou, and C. Chen, "A Monolithic AlGaN/GaN HEMT VCO Using BST Thin-Film Varactor," IEEE Transactions on Microwave Theory and Techniques, vol. 60, no. 11, pp. 3413-3419, Nov. 2012.

[125] H. Liu, X. Zhu, C. C. Boon, X. Yi, M. Mao, and W. Yang, "Design of Ultra-Low Phase Noise and High Power Integrated Oscillator in GaN-on-SiC HEMT Technology," IEEE Microwave and Wireless Components Letters, vol. 24, no. 2, pp. 120-122, Feb. 2014.

[126] H. Xu, C. Sanabria, S. Heikman, S. Keller, U. K. Mishra, and R. A. York, "High power GaN oscillators using field-plated HEMT structure," in IEEE MTT-S International Microwave Symposium Digest, 2005., 2005, p. 4 pp.-pp.

[127] H. Kao, B.-W. Wang, C.-S. Yeh, C.-L. Cho, B.-H. Wei, and H.-C. Chiu, "High Power and Low Phase Noise MMIC VCO Using 0.35m GaN-on-Si HEMT," International Journal of Computer Theory and Engineering, pp. 910-913, 2013.

[128] T. N. T. Do, S. Lai, M. Horberg, H. Zirath, and D. Kuylenstierna, "A MMIC GaN HEMT Voltage-Controlled-Oscillator with High Tuning Linearity and Low 
Phase Noise," in 2015 IEEE Compound Semiconductor Integrated Circuit Symposium (CSICS), 2015, pp. 1-4.

[129] K. V, V. Tilak, H. Kim, R. Thompson, T. Prunty, J. Smart, L. F. Eastman, and J. R. Shealy, "High power monolithic AlGaN/GaN HEMT oscillator," in Gallium Arsenide Integrated Circuit (GaAs IC) Symposium, 2002. 24th Annual Technical Digest, 2002, pp. 251-254.

[130] Z. Q. Cheng, Y. Cai, J. Liu, Y. Zhou, K. M. Lau, and K. J. Chen, "A Low PhaseNoise -Band MMIC VCO Using High-Linearity and Low-Noise Composite-Channel HEMTs," IEEE Transactions on Microwave Theory and Techniques, vol. 55, no. 1, pp. 23-29, Jan. 2007.

[131] G. Soubercaze-Pun, J. G. Tartarin, L. Bary, J. Rayssac, E. Morvan, B. Grimbert, S. L. Delage, J. c D. Jaeger, and J. Graffeuil, "Design of a X-band GaN oscillator: from the low frequency noise device characterization and large signal modeling to circuit design," in 2006 IEEE MTT-S International Microwave Symposium Digest, 2006, pp. 747-750.

[132] S. Lai, D. Kuylenstierna, M. Özen, M. Hörberg, N. Rorsman, I. Angelov, and H. Zirath, "Low Phase Noise GaN HEMT Oscillators With Excellent Figures of Merit," IEEE Microwave and Wireless Components Letters, vol. 24, no. 6, pp. 412414, Jun. 2014.

[133] M. Hörberg and D. Kuylenstierna, "Low phase noise power-efficient MMIC GaN-HEMT oscillator at $15 \mathrm{GHz}$ based on a quasi-lumped on-chip resonator," in 2015 IEEE MTT-S International Microwave Symposium, 2015, pp. 1-4.

[134] M. Hörberg, T. Emanuelsson, S. Lai, T. N. D. Thanh, H. Zirath, and D. Kuylenstierna, "Phase-Noise Analysis of an X-Band Ultra-Low Phase-Noise GaN HEMT Based Cavity Oscillator," IEEE Transactions on Microwave Theory and Techniques, vol. 63, no. 8, pp. 2619-2629, Aug. 2015.

[135] X. Lan, M. Wojtowicz, I. Smorchkova, R. Coffie, R. Tsai, B. Heying, M. Truong, F. Fong, M. Kintis, C. Namba, A. Oki, and T. Wong, "A Q-band low phase noise monolithic AlGaN/GaN HEMT VCO," IEEE Microwave and Wireless Components Letters, vol. 16, no. 7, pp. 425-427, Jul. 2006.

[136] X. Lan, M. Wojtowicz, M. Truong, F. Fong, M. Kintis, B. Heying, I. Smorchkova, and Y. C. Chen, "A V-Band Monolithic AlGaN/GaN VCO," IEEE Microwave and Wireless Components Letters, vol. 18, no. 6, pp. 407-409, Jun. 2008.

[137] J. B. Shealy, T. Jackson, A. Rachlin, M. Poulton, N. Bukhari, K. Ditzler, X. Gong, L. Sumpter, and D. Weeks, "A 2 watt Ku-band linear (multi-carrier) transmit module for VSAT applications," in Microwave Symposium Digest, 1999 IEEE MTTS International, 1999, vol. 3, pp. 1055-1058 vol.3.

[138] H. Zirath, R. Kozhuharov, and M. Ferndahl, "Balanced Colpitt oscillator MMICs designed for ultra-low phase noise," IEEE Journal of Solid-State Circuits, vol. 40, no. 10, pp. 2077-2086, Oct. 2005. 
[139] Cree's CGHV1J025D datasheet.

[140] M. Caillet, "Etude et Conception d'un Système Radar Courte Portée et d'Antennes Reconfigurables en Bande Millimétrique," PhD Dissertation. University of Rennes 1, Rennes, France. November, 2006.

[141] S. Sufyar, C. Delaveaud, and R. Staraj, "A frequency agility technique on a miniature omnidirectional antenna," in Proceedings of the Fourth European Conference on Antennas and Propagation, 2010, pp. 1-4.

[142] S. K. Dhar, O. Hammi, M. S. Sharawi, and F. M. Ghannouchi, "Power amplifier based integrated and miniaturized active antenna," in 2015 9th European Conference on Antennas and Propagation (EuCAP), 2015, pp. 1-4.

[143] V. K. Varadan, K. A. Jose, and V. V. Varadan, "Design and development of electronically tunable microstrip antennas," Smart Mater. Struct., vol. 8, no. 2, p. 238, 1999.

[144] R. B. Whatley, Z. Zhou, and K. L. Melde, "Reconfigurable RF impedance tuner for match control in broadband wireless devices," IEEE Transactions on Antennas and Propagation, vol. 54, no. 2, pp. 470-478, Feb. 2006.

[145] A. Hamdoun, L. Roy, M. Himdi, and O. Lafond, "Characterization of GaNbased HEMTs as varactor diode devices," in Microwave Integrated Circuits Conference (EuMIC), 2015 10th European, 2015, pp. 417-420.

[146] "An Update on New Tunable Technologies", From July 2010 High Frequency Electronics. Copyright (C) 2010 Summit Technical Media, LLC.

[147] A. Martin, X. Castel, O. Lafond, and M. Himdi, "Optically transparent frequency-agile antenna for X-band applications," Electronics Letters, vol. 51, no. 16, pp. 1231-1233, 2015.

[148] J. S. McLean, "A re-examination of the fundamental limits on the radiation Q of electrically small antennas," IEEE Transactions on Antennas and Propagation, vol. 44, no. 5, p. 672-, May 1996.

[149] A. Hamdoun, L. Roy, M. Himdi, and O. Lafond, "Characterisation and analytical modelling of GaN HEMT-based varactor diodes," Electronics Letters, vol. 51, no. 23, pp. 1930-1932, 2015.

[150] A. Hamdoun, L. Roy, M. Himdi, and O. Lafond, "Characterization of GaNbased HEMTs as varactor diode devices," in Microwave Integrated Circuits Conference (EuMIC), 2015 10th European, 2015, pp. 417-420.

[151] A. Hamdoun, L. Roy, M. Himdi and O. Lafond, "Large Signal Analytic Modeling of HEMT-based Varactor Diodes," Submitted to IEEE Transactions on Electron Devices IEEE. September 2016.

[152] A. Hamdoun, L. Roy, M. Himdi and O. Lafond, "Switched Beam Patch Array Antenna Using SPDT GaN HEMT Switches, "in European Conference on Antennas and Propagatione (EuCAP), 11th European, Paris 2015. 